Supplement of Hydrol. Earth Syst. Sci., 21, 3937-3952, 2017

https://doi.org/10.5194/hess-21-3937-2017-supplement

(c) Author(s) 2017. This work is distributed under

the Creative Commons Attribution 3.0 License.

(c) (1)

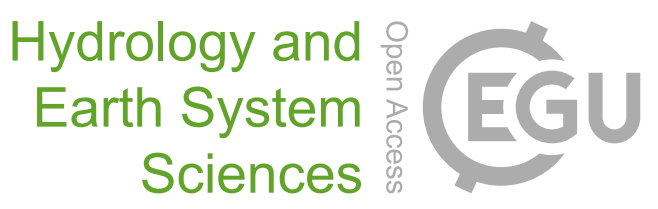

Supplement of

\title{
Impact of model structure on flow simulation and hydrological realism: from a lumped to a semi-distributed approach
}

Federico Garavaglia et al.

Correspondence to: Federico Garavaglia (federico.garavaglia@edf.fr)

The copyright of individual parts of the supplement might differ from the CC BY 3.0 License. 


\section{S1 Catchments features}

This section details the dataset of the 50 catchments. Figure S1 shows the localization of the studied catchments. The catchments IDs are highlighted in figure S1. Table S1 presents the main features of the catchments dataset, including name, geographical position, area, elevation range, slope, annual $\mathrm{P}$, annual PET, annual $\mathrm{Q}$, time step, modeling periods $\mathrm{P} 1$ and $\mathrm{P} 2$.

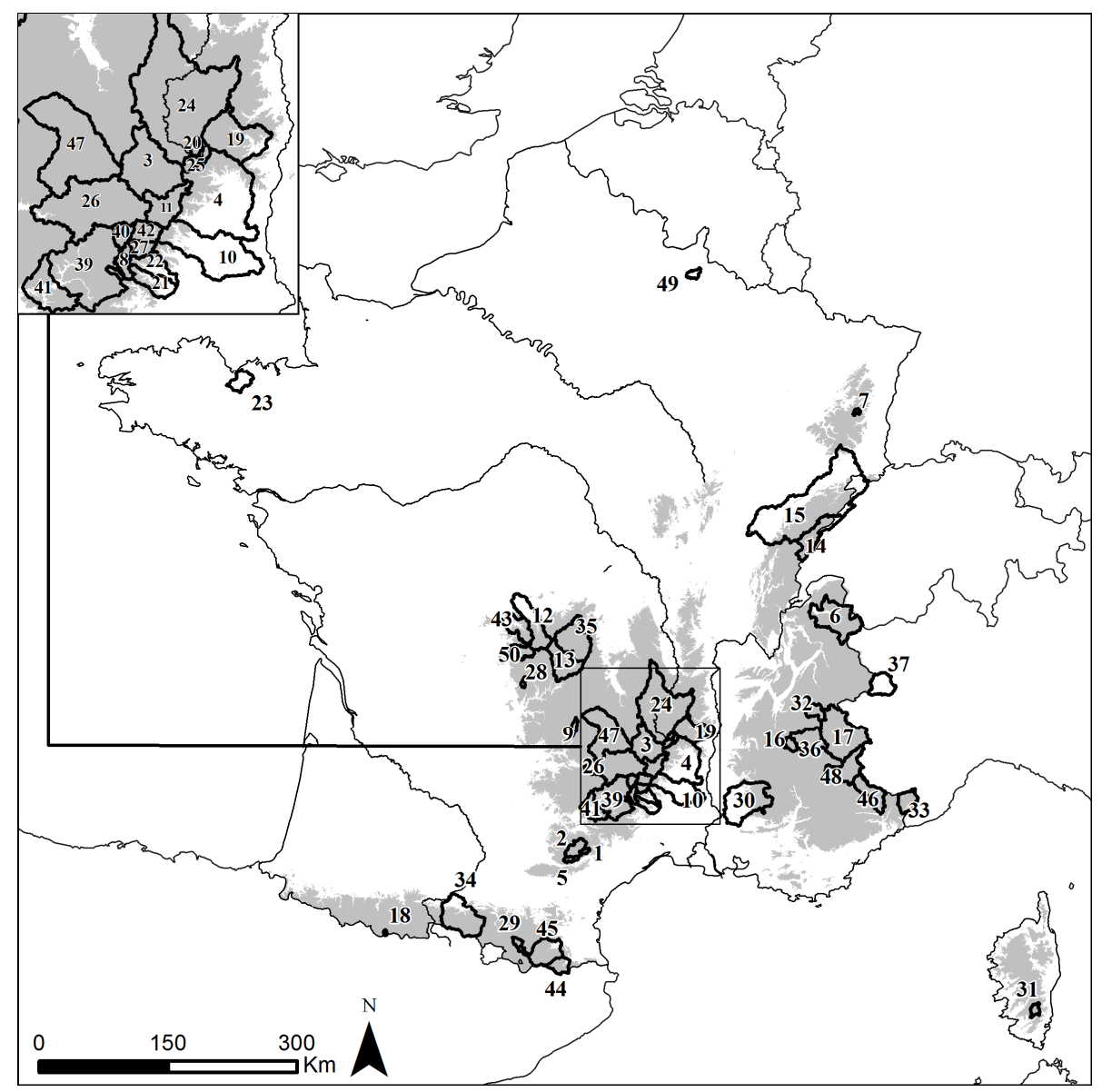

Figure S1: Localization of studied catchment, the catchment IDs are highlighted. 


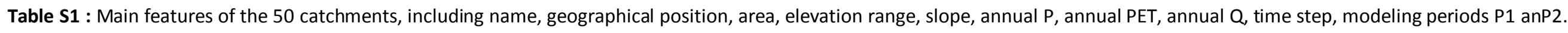

\begin{tabular}{|c|c|c|c|c|c|c|c|c|c|c|c|c|c|c|c|c|}
\hline ID & BV & $\begin{array}{l}\text { Xe } \\
{[\mathrm{m}]}\end{array}$ & $\begin{array}{l}\text { Ye } \\
{[\mathrm{m}]}\end{array}$ & $\begin{array}{c}\text { Surface } \\
{\left[\mathrm{km}^{2}\right]}\end{array}$ & $\begin{array}{l}\text { Latitude } \\
\text { [degree] }\end{array}$ & $\begin{array}{c}\text { Zmin } \\
{[\mathrm{m}]}\end{array}$ & $\begin{array}{l}\mathrm{Z} 50 \\
{[\mathrm{~m}]}\end{array}$ & $\begin{array}{c}\text { Zmax } \\
{[\mathrm{m}]}\end{array}$ & $\begin{array}{c}\text { Slope } \\
\text { [degree] }\end{array}$ & $\begin{array}{c}\text { Annual_P } \\
\text { [mm] }\end{array}$ & $\begin{array}{c}\text { Annual_ETP } \\
{[\mathrm{mm}]}\end{array}$ & $\begin{array}{c}\text { Annual_Q } \\
\text { [mm] }\end{array}$ & $\begin{array}{c}\text { Time step } \\
\text { [hour] }\end{array}$ & $\begin{array}{l}\text { Modelling } \\
\text { Period } 1\end{array}$ & $\begin{array}{c}\text { Modelling } \\
\text { Period } 2\end{array}$ & Mountain region \\
\hline 1 & Agout@Fraisse & 637989 & 1845758 & 46.8 & 43.4 & 793 & 999 & 1149 & 5.05 & 1573 & 660 & 1073 & 24 & $1973-1981$ & $1981-1988$ & Massif Central \\
\hline 2 & Agout@LaRaviege & 621096 & 1843766 & 370.8 & 43.6 & 659 & 904 & 1259 & 6.37 & 1510 & 657 & 969 & 24 & $1973-1980$ & $1980-1987$ & Massif Central \\
\hline 3 & Allier@Poutes & 705537 & 1994942 & 1016.1 & 46.5 & 659 & 1146 & 1528 & 5.41 & 1046 & 604 & 496 & 24 & $1988-1996$ & $1996-2004$ & Massif Central \\
\hline 4 & Ardeche@Sauze & 776846 & 1926227 & 2266.5 & 44.1 & 49 & 599 & 1671 & 9.51 & 1574 & 757 & 838 & 24 & 1961 - 1985 & $1985-2008$ & Massif Central \\
\hline 5 & Arn@Taillades & 619482 & 1836883 & 82.4 & 43.5 & 681 & 880 & 1054 & 4.08 & 1630 & 683 & 1045 & 24 & $1987-1997$ & $1997-2007$ & Massif Central \\
\hline 6 & Arve@Arthaz & 904338 & 2135177 & 1638.2 & 46.2 & 433 & 1504 & 4800 & 19.61 & 1552 & 569 & 1438 & 24 & $1986-1993$ & $1993-2000$ & Alps \\
\hline 7 & Behine@LaPoutroie & 953246 & 2358462 & 38.4 & 48.2 & 406 & 856 & 1215 & 12.58 & 1316 & 611 & 805 & 24 & $1984-1989$ & $1989-1993$ & Vosges \\
\hline 8 & Breze@Meyrueis & 688063 & 1909099 & 33.7 & 44.0 & 703 & 1075 & 1544 & 13.41 & 1444 & 622 & 843 & 24 & $1985-1996$ & $1996-2006$ & Massif Central \\
\hline 9 & Bromme@Brommat & 627263 & 1980786 & 106.3 & 44.8 & 660 & 978 & 1610 & 5.70 & 1422 & 696 & 972 & 24 & $1989-1995$ & $1995-2000$ & Massif Central \\
\hline 11 & Chassezac@SteMarguerite & 731606 & 1942481 & 414.1 & 44.3 & 329 & 1055 & 1671 & 11.03 & 1611 & 580 & 1094 & 24 & $1968-1987$ & $1987-2006$ & Massif Central \\
\hline 12 & Creuse@Age & 559016 & 2144605 & 1121.8 & 46.0 & 266 & 553 & 948 & 3.57 & 1072 & 650 & 416 & 24 & $1960-1983$ & $1983-2005$ & Massif Central \\
\hline 13 & Dordogne@Bort & 612697 & 2046020 & 1012.6 & 45.4 & 528 & 836 & 1774 & 4.15 & 1327 & 621 & 724 & 24 & $1979-1992$ & $1992-2005$ & Massif Central \\
\hline 14 & Doubs@Brenet & 931797 & 2240815 & 941.0 & 47.4 & 768 & 1000 & 1438 & 5.09 & 1445 & 698 & 822 & 24 & $1962-1983$ & $1983-2004$ & Jura \\
\hline 15 & Doubs@Neublans & 829420 & 2218279 & 7366.2 & 46.9 & 184 & 580 & 1438 & 4.55 & 1422 & 632 & 764 & 24 & $1967-1984$ & $1984-2001$ & Jura \\
\hline 16 & Drac@Sautet & 882507 & 1985816 & 990.6 & 44.5 & 780 & 1742 & 3512 & 20.84 & 1421 & 579 & 1049 & 24 & $1969-1984$ & $1984-1999$ & Alps \\
\hline 17 & Durance@Clapiere & 930627 & 1959457 & 2174.2 & 44.6 & 790 & 2113 & 3980 & 21.56 & 1159 & 444 & 725 & 24 & $1982-1994$ & $1994-2005$ & Alps \\
\hline 18 & Eyrieux@Pontpierre & 785116 & 1983533 & 770.1 & 44.8 & 141 & 797 & 1710 & 10.98 & 1325 & 711 & 625 & 24 & $1998-2006$ & $2006-2013$ & Massif Central \\
\hline 19 & Gage@Gagell & 739315 & 1979094 & 42.4 & 44.5 & 1011 & 1254 & 1592 & 6.36 & 1506 & 586 & 1107 & 6 & $1992-1999$ & $1999-2005$ & Massif Central \\
\hline 20 & Gardon@Corbes & 730274 & 1898530 & 262.3 & 44.1 & 145 & 531 & 1181 & 12.02 & 1369 & 729 & 801 & 8 & $1986-1997$ & $1997-2007$ & Massif Central \\
\hline 23 & Goussant@PontRolland & 233822 & 2402559 & 422.5 & 48.5 & 31 & 109 & 338 & 1.54 & 824 & 685 & 224 & 24 & $1993-1999$ & $1999-2004$ & Britanny region \\
\hline 24 & Loire@Basset & 739771 & 2034325 & 3261.8 & 45.3 & 446 & 965 & 1701 & 5.49 & 993 & 731 & 419 & 24 & $1956-1983$ & $1983-2010$ & Massif Central \\
\hline 25 & Loire@LaPalisse & 738771 & 1977633 & 130.9 & 44.5 & 1003 & 1245 & 1570 & 6.02 & 1778 & 585 & 1398 & 6 & $1992-1998$ & $1998-2004$ & Massif Central \\
\hline 26 & Lot@Castelnau & 642336 & 1945506 & 1628.2 & 44.3 & 416 & 981 & 1700 & 7.24 & 1042 & 629 & 457 & 24 & $1982-1994$ & $1994-2005$ & Massif Central \\
\hline 27 & Mimente@Florac & 701260 & 1924715 & 126.1 & 44.0 & 559 & 933 & 1409 & 11.09 & 1453 & 650 & 840 & 24 & $1985-1996$ & $1996-2006$ & Massif Central \\
\hline 28 & Montane@Eyrein & 568891 & 2038403 & 43.2 & 45.3 & 553 & 624 & 803 & 3.09 & 1497 & 662 & 931 & 24 & $1958-1979$ & $1979-1999$ & Massif Central \\
\hline 29 & Oriege@Campauleil & 560272 & 1745078 & 89.4 & 42.7 & 828 & 1829 & 2748 & 24.50 & 1928 & 479 & 1522 & 24 & $1960-1971$ & $1971-1982$ & Pyrenees \\
\hline 30 & Ouveze@Bedarrides & 805366 & 1896400 & 1841.9 & 44.2 & 25 & 510 & 1895 & 6.70 & 868 & 798 & 364 & 24 & $1996-2000$ & $2000-2004$ & Alps \\
\hline 31 & Rizzanese@Barrage & 1165253 & 1660880 & 116.2 & 41.6 & 554 & 1086 & 2028 & 11.92 & 1309 & 892 & 778 & 6 & $1993-2000$ & $2000-2007$ & Corsica island \\
\hline 32 & Romanche@Chambon & 899366 & 2012160 & 256.6 & 45.2 & 1063 & 2365 & 3797 & 24.19 & 1468 & 427 & 1020 & 24 & $1985-1995$ & $1995-2005$ & Alps \\
\hline 33 & Roya@Breil & 1015841 & 1895339 & 444.4 & 43.5 & 316 & 1475 & 2893 & 21.48 & 1307 & 650 & 776 & 24 & $1987-1996$ & $1996-2005$ & Alps \\
\hline 34 & Salat@Roquefort & 488576 & 1795992 & 1580.2 & 42.9 & 269 & 982 & 2807 & 15.43 & 1468 & 713 & 786 & 24 & $1993-1998$ & $1998-2003$ & Pyrenees \\
\hline 35 & Sioule@Fades & 635772 & 2108190 & 1290.6 & 45.9 & 508 & 778 & 1508 & 3.52 & 1101 & 727 & 435 & 24 & $1956-1982$ & $1982-2008$ & Massif Central \\
\hline 36 & Souloise@Infernet & 882093 & 1980560 & 163.0 & 44.8 & 854 & 1685 & 2737 & 16.94 & 1636 & 677 & 1224 & 24 & $1991-1999$ & $1999-2006$ & Alps \\
\hline 37 & Stura@Lanzo & 1003561 & 2042987 & 579.2 & 45.1 & 462 & 1785 & 3625 & 22.98 & 1497 & 478 & 1080 & 24 & $1954-1963$ & $1963-1972$ & Alps \\
\hline 38 & Tarn@Cocures & 702310 & 1927978 & 189.4 & 44.0 & 583 & 1192 & 1697 & 9.11 & 1482 & 627 & 962 & 24 & $1985-1996$ & $1996-2006$ & Massif Central \\
\hline 39 & Tarn@Millau & 658957 & 1899147 & 2085.7 & 44.0 & 349 & 896 & 1697 & 8.69 & 1213 & 642 & 630 & 24 & $1980-1995$ & $1995-2010$ & Massif Central \\
\hline 40 & Tarn@Montbrun & 692563 & 1926599 & 588.6 & 44.0 & 487 & 1017 & 1700 & 10.55 & 1353 & 619 & 861 & 24 & $1985-1996$ & $1996-2006$ & Massif Central \\
\hline 41 & Tarn@Pinet & 637927 & 1896585 & 2624.6 & 44.0 & 315 & 848 & 1697 & 8.29 & 1201 & 615 & 598 & 24 & $1980-1988$ & $1988-1995$ & Massif Central \\
\hline 42 & Tarnon@Florac & 700826 & 1924522 & 133.1 & 44.0 & 557 & 980 & 1550 & 11.74 & 1392 & 620 & 813 & 24 & $1985-1996$ & $1996-2006$ & Massif Central \\
\hline 46 & Tinee@PontLune & 988003 & 1893796 & 707.0 & 44.2 & 317 & 1750 & 2998 & 22.45 & 1260 & 563 & 643 & 24 & $1981-1992$ & $1992-2002$ & Alps \\
\hline 47 & Truyere@Grandval & 658325 & 1991594 & 1788.8 & 44.5 & 723 & 1061 & 1500 & 4.07 & 997 & 581 & 454 & 24 & $1973-1990$ & $1990-2006$ & Massif Central \\
\hline 48 & Ubaye@RocheRousse & 923493 & 1947189 & 946.6 & 44.4 & 804 & 2086 & 3317 & 20.78 & 1098 & 447 & 671 & 24 & $1982-1994$ & $1994-2005$ & Alps \\
\hline 49 & Vence@Francheville & 771573 & 2528249 & 125.6 & 49.4 & 152 & 219 & 335 & 3.04 & 1022 & 653 & 577 & 24 & $1974-1985$ & $1985-1995$ & Ardennes region \\
\hline 50 & Vienne@Bussy & 552421 & 2082789 & 379.2 & 46.9 & 407 & 691 & 951 & 4.64 & 1328 & 713 & 755 & 24 & $1983-1995$ & $1995-2006$ & Massif Central \\
\hline
\end{tabular}




\section{S2 Model comparison over calibration periods}

This section details the performance of MORDOR V0, V1 and V2 over the calibration periods P1 and P2. For the three considered models, tables S2 and S3 show the values of the individual associated calibration metrics (KGE(Q), KGE(Qsea) and KGE(FDC)) for all the catchments over the calibration period P1 (table S2) and the calibration period P2 (table S3). The following figures show, for the 50 catchments, the observed hydrograph (year by year) and those modeled by MORDOR V0, V1 and SD (calibration mode). 
Table S2: The values of the individual associated calibration metrics (KGE(Q), KGE(Qsea) and KGE(FDC)) for MORDOR V0, V1 and SD for all the catchments over the period P1

\begin{tabular}{|c|c|c|c|c|c|c|c|c|c|c|}
\hline \multirow{2}{*}{ ID } & \multirow{2}{*}{ BV } & \multicolumn{3}{|c|}{ MORDOR V0 } & \multicolumn{3}{|c|}{ MORDOR V1 } & \multicolumn{3}{|c|}{ MORDOR SD } \\
\hline & & KGE(Q) & KGE(Qsea) & KGE(FDC) & KGE(Q) & KGE(Qsea) & KGE(FDC) & KGE(Q) & KGE(Qsea) & KGE(FDC) \\
\hline 1 & Agout@Fraisse & 0.948 & 0.995 & 0.898 & 0.963 & 0.991 & 0.914 & 0.955 & 0.974 & 0.900 \\
\hline 2 & Agout@LaRaviege & 0.911 & 0.978 & 0.953 & 0.910 & 0.973 & 0.950 & 0.906 & 0.969 & 0.954 \\
\hline 3 & Allier@Poutes & 0.960 & 0.889 & 0.925 & 0.969 & 0.885 & 0.939 & 0.960 & 0.881 & 0.941 \\
\hline 4 & Ardeche@Sauze & 0.928 & 0.958 & 0.924 & 0.951 & 0.964 & 0.923 & 0.948 & 0.964 & 0.922 \\
\hline 5 & Arn@Taillades & 0.885 & 0.938 & 0.987 & 0.909 & 0.944 & 0.989 & 0.923 & 0.938 & 0.989 \\
\hline 6 & Arve@Arthaz & 0.984 & 0.817 & 0.918 & 0.991 & 0.812 & 0.926 & 0.981 & 0.792 & 0.929 \\
\hline 7 & Behine@LaPoutroie & 0.961 & 0.962 & 0.903 & 0.957 & 0.965 & 0.882 & 0.954 & 0.939 & 0.908 \\
\hline 8 & Breze@Meyrueis & 0.879 & 0.926 & 0.984 & 0.847 & 0.943 & 0.983 & 0.853 & 0.939 & 0.984 \\
\hline 9 & Bromme@Brommat & 0.984 & 0.841 & 0.943 & 0.985 & 0.880 & 0.940 & 0.986 & 0.883 & 0.939 \\
\hline 10 & Ceze@Bagnols & 0.947 & 0.943 & 0.887 & 0.954 & 0.917 & 0.892 & 0.959 & 0.940 & 0.880 \\
\hline 11 & Chassezac@SteMarguerite & 0.903 & 0.939 & 0.959 & 0.924 & 0.944 & 0.956 & 0.911 & 0.950 & 0.957 \\
\hline 12 & Creuse@Age & 0.980 & 0.888 & 0.946 & 0.983 & 0.901 & 0.948 & 0.984 & 0.896 & 0.944 \\
\hline 13 & Dordogne@Bort & 0.913 & 0.924 & 0.889 & 0.926 & 0.929 & 0.879 & 0.934 & 0.927 & 0.868 \\
\hline 14 & Doubs@Brenet & 0.874 & 0.909 & 0.976 & 0.901 & 0.910 & 0.985 & 0.874 & 0.920 & 0.981 \\
\hline 15 & Doubs@Neublans & 0.974 & 0.898 & 0.962 & 0.981 & 0.874 & 0.967 & 0.981 & 0.895 & 0.966 \\
\hline 16 & Drac@Sautet & 0.940 & 0.974 & 0.916 & 0.927 & 0.956 & 0.901 & 0.933 & 0.985 & 0.899 \\
\hline 17 & Durance@Clapiere & 0.900 & 0.926 & 0.975 & 0.895 & 0.933 & 0.983 & 0.903 & 0.939 & 0.981 \\
\hline 18 & Eyrieux@Pontpierre & 0.982 & 0.848 & 0.951 & 0.979 & 0.862 & 0.963 & 0.978 & 0.843 & 0.961 \\
\hline 19 & Gage@Gagell & 0.931 & 0.971 & 0.921 & 0.915 & 0.974 & 0.937 & 0.894 & 0.970 & 0.924 \\
\hline 20 & Gardon@Corbes & 0.882 & 0.906 & 0.984 & 0.832 & 0.919 & 0.982 & 0.787 & 0.935 & 0.982 \\
\hline 21 & Gardon@Generargues & 0.968 & 0.909 & 0.928 & 0.967 & 0.921 & 0.958 & 0.960 & 0.906 & 0.957 \\
\hline 22 & GaveEstaube@Gloriettes & 0.933 & 0.979 & 0.871 & 0.942 & 0.984 & 0.886 & 0.935 & 0.980 & 0.871 \\
\hline 23 & Goussant@PontRolland & 0.849 & 0.947 & 0.967 & 0.841 & 0.962 & 0.961 & 0.823 & 0.958 & 0.965 \\
\hline 24 & Loire@Basset & 0.966 & 0.815 & 0.931 & 0.969 & 0.832 & 0.935 & 0.969 & 0.815 & 0.935 \\
\hline 25 & Loire@LaPalisse & 0.940 & 0.943 & 0.884 & 0.937 & 0.944 & 0.896 & 0.912 & 0.958 & 0.891 \\
\hline 26 & Lot@Castelnau & 0.838 & 0.946 & 0.958 & 0.853 & 0.949 & 0.959 & 0.826 & 0.950 & 0.961 \\
\hline 27 & Mimente@Florac & 0.954 & 0.925 & 0.942 & 0.967 & 0.951 & 0.947 & 0.948 & 0.959 & 0.953 \\
\hline 28 & Montane@Eyrein & 0.918 & 0.975 & 0.895 & 0.926 & 0.982 & 0.917 & 0.928 & 0.982 & 0.929 \\
\hline 29 & Oriege@Campauleil & 0.820 & 0.944 & 0.986 & 0.819 & 0.953 & 0.989 & 0.826 & 0.951 & 0.987 \\
\hline 30 & Ouveze@Bedarrides & 0.971 & 0.863 & 0.958 & 0.977 & 0.926 & 0.946 & 0.978 & 0.929 & 0.942 \\
\hline 31 & Rizzanese@Barrage & 0.948 & 0.971 & 0.897 & 0.951 & 0.973 & 0.854 & 0.943 & 0.973 & 0.815 \\
\hline 32 & Romanche@Chambon & 0.932 & 0.948 & 0.970 & 0.941 & 0.956 & 0.975 & 0.912 & 0.945 & 0.971 \\
\hline 33 & Roya@Breil & 0.975 & 0.939 & 0.879 & 0.978 & 0.931 & 0.887 & 0.977 & 0.913 & 0.884 \\
\hline 34 & Salat@Roquefort & 0.952 & 0.990 & 0.872 & 0.937 & 0.991 & 0.892 & 0.953 & 0.991 & 0.899 \\
\hline 35 & Sioule@Fades & 0.924 & 0.906 & 0.957 & 0.928 & 0.906 & 0.969 & 0.930 & 0.903 & 0.969 \\
\hline 36 & Souloise@Infernet & 0.992 & 0.883 & 0.918 & 0.992 & 0.869 & 0.910 & 0.991 & 0.858 & 0.949 \\
\hline 37 & Stura@Lanzo & 0.916 & 0.988 & 0.841 & 0.951 & 0.988 & 0.796 & 0.945 & 0.986 & 0.862 \\
\hline 38 & Tarn@Cocures & 0.904 & 0.927 & 0.932 & 0.930 & 0.934 & 0.946 & 0.935 & 0.931 & 0.954 \\
\hline 39 & Tarn@Millau & 0.981 & 0.793 & 0.927 & 0.984 & 0.799 & 0.938 & 0.985 & 0.806 & 0.934 \\
\hline 40 & Tarn@Montbrun & 0.921 & 0.973 & 0.926 & 0.936 & 0.980 & 0.942 & 0.918 & 0.977 & 0.924 \\
\hline 41 & Tarn@Pinet & 0.917 & 0.922 & 0.982 & 0.922 & 0.902 & 0.980 & 0.923 & 0.900 & 0.981 \\
\hline 42 & Tarnon@Florac & 0.962 & 0.775 & 0.946 & 0.972 & 0.731 & 0.946 & 0.966 & 0.806 & 0.957 \\
\hline 43 & Taurion@RocheTalamie & 0.934 & 0.956 & 0.906 & 0.956 & 0.954 & 0.885 & 0.951 & 0.948 & 0.906 \\
\hline 44 & Tech@Reynes & 0.929 & 0.937 & 0.993 & 0.950 & 0.939 & 0.991 & 0.955 & 0.949 & 0.976 \\
\hline 45 & Tet@Vinca & 0.985 & 0.896 & 0.932 & 0.990 & 0.876 & 0.935 & 0.987 & 0.855 & 0.934 \\
\hline 46 & Tinee@PontLune & 0.924 & 0.989 & 0.879 & 0.949 & 0.983 & 0.873 & 0.958 & 0.994 & 0.840 \\
\hline 47 & Truyere@Grandval & 0.899 & 0.864 & 0.985 & 0.901 & 0.899 & 0.987 & 0.923 & 0.895 & 0.987 \\
\hline 48 & Ubaye@RocheRousse & 0.991 & 0.798 & 0.957 & 0.990 & 0.802 & 0.953 & 0.966 & 0.832 & 0.962 \\
\hline 49 & Vence@Francheville & 0.945 & 0.919 & 0.903 & 0.934 & 0.960 & 0.931 & 0.966 & 0.951 & 0.924 \\
\hline 50 & Vienne@Bussy & 0.871 & 0.900 & 0.980 & 0.846 & 0.904 & 0.985 & 0.887 & 0.906 & 0.982 \\
\hline
\end{tabular}


Table S3: The values of the individual associated calibration metrics (KGE(Q), KGE(Qsea) and KGE(FDC)) for MORDOR V0, V1 and SD for all the catchments over the period P3

\begin{tabular}{|c|c|c|c|c|c|c|c|c|c|c|}
\hline \multirow{2}{*}{ ID } & \multirow{2}{*}{ BV } & \multicolumn{3}{|c|}{ MORDOR V0 } & \multicolumn{3}{|c|}{ MORDOR V1 } & \multicolumn{3}{|c|}{ MORDOR SD } \\
\hline & & KGE(Q) & KGE(Qsea) & KGE(FDC) & KGE(Q) & KGE(Qsea) & KGE(FDC) & KGE(Q) & KGE(Qsea) & KGE(FDC) \\
\hline 1 & Agout@Fraisse & 0.977 & 0.846 & 0.922 & 0.981 & 0.833 & 0.921 & 0.976 & 0.842 & 0.932 \\
\hline 2 & Agout@LaRaviege & 0.938 & 0.981 & 0.880 & 0.947 & 0.982 & 0.871 & 0.941 & 0.980 & 0.910 \\
\hline 3 & Allier@Poutes & 0.878 & 0.940 & 0.986 & 0.901 & 0.951 & 0.988 & 0.869 & 0.950 & 0.988 \\
\hline 4 & Ardeche@Sauze & 0.981 & 0.866 & 0.937 & 0.986 & 0.861 & 0.941 & 0.987 & 0.860 & 0.945 \\
\hline 5 & Arn@Taillades & 0.962 & 0.958 & 0.916 & 0.973 & 0.963 & 0.907 & 0.969 & 0.958 & 0.900 \\
\hline 6 & Arve@Arthaz & 0.908 & 0.932 & 0.975 & 0.931 & 0.939 & 0.972 & 0.857 & 0.919 & 0.978 \\
\hline 7 & Behine@LaPoutroie & 0.963 & 0.826 & 0.950 & 0.960 & 0.828 & 0.948 & 0.953 & 0.785 & 0.961 \\
\hline 8 & Breze@Meyrueis & 0.966 & 0.951 & 0.905 & 0.975 & 0.963 & 0.901 & 0.976 & 0.964 & 0.884 \\
\hline 9 & Bromme@Brommat & 0.893 & 0.910 & 0.978 & 0.891 & 0.900 & 0.979 & 0.903 & 0.918 & 0.976 \\
\hline 10 & Ceze@Bagnols & 0.971 & 0.801 & 0.915 & 0.977 & 0.770 & 0.917 & 0.977 & 0.820 & 0.927 \\
\hline 11 & Chassezac@SteMarguerite & 0.937 & 0.963 & 0.788 & 0.955 & 0.970 & 0.783 & 0.958 & 0.971 & 0.795 \\
\hline 12 & Creuse@Age & 0.908 & 0.895 & 0.958 & 0.923 & 0.898 & 0.955 & 0.910 & 0.901 & 0.953 \\
\hline 13 & Dordogne@Bort & 0.956 & 0.788 & 0.947 & 0.975 & 0.801 & 0.967 & 0.974 & 0.804 & 0.963 \\
\hline 14 & Doubs@Brenet & 0.907 & 0.982 & 0.898 & 0.934 & 0.967 & 0.922 & 0.928 & 0.980 & 0.926 \\
\hline 15 & Doubs@Neublans & 0.910 & 0.897 & 0.978 & 0.917 & 0.893 & 0.977 & 0.905 & 0.923 & 0.977 \\
\hline 16 & Drac@Sautet & 0.983 & 0.844 & 0.941 & 0.962 & 0.840 & 0.961 & 0.971 & 0.867 & 0.958 \\
\hline 17 & Durance@Clapiere & 0.939 & 0.970 & 0.822 & 0.941 & 0.972 & 0.854 & 0.938 & 0.971 & 0.859 \\
\hline 18 & Eyrieux@Pontpierre & 0.886 & 0.948 & 0.967 & 0.887 & 0.948 & 0.977 & 0.874 & 0.929 & 0.974 \\
\hline 19 & Gage@Gagell & 0.956 & 0.861 & 0.965 & 0.943 & 0.865 & 0.960 & 0.922 & 0.859 & 0.965 \\
\hline 20 & Gardon@Corbes & 0.949 & 0.975 & 0.920 & 0.947 & 0.974 & 0.923 & 0.942 & 0.976 & 0.925 \\
\hline 21 & Gardon@Generargues & 0.893 & 0.935 & 0.968 & 0.898 & 0.930 & 0.979 & 0.900 & 0.940 & 0.974 \\
\hline 22 & GaveEstaube@Gloriettes & 0.952 & 0.932 & 0.948 & 0.960 & 0.916 & 0.954 & 0.955 & 0.899 & 0.954 \\
\hline 23 & Goussant@PontRolland & 0.947 & 0.971 & 0.896 & 0.952 & 0.973 & 0.891 & 0.951 & 0.971 & 0.896 \\
\hline 24 & Loire@Basset & 0.894 & 0.929 & 0.948 & 0.905 & 0.925 & 0.955 & 0.882 & 0.942 & 0.957 \\
\hline 25 & Loire@LaPalisse & 0.954 & 0.756 & 0.943 & 0.945 & 0.745 & 0.944 & 0.915 & 0.799 & 0.944 \\
\hline 26 & Lot@Castelnau & 0.923 & 0.981 & 0.873 & 0.933 & 0.986 & 0.863 & 0.927 & 0.988 & 0.891 \\
\hline 27 & Mimente@Florac & 0.889 & 0.932 & 0.987 & 0.902 & 0.958 & 0.991 & 0.850 & 0.948 & 0.992 \\
\hline 28 & Montane@Eyrein & 0.940 & 0.855 & 0.951 & 0.947 & 0.856 & 0.956 & 0.947 & 0.849 & 0.956 \\
\hline 29 & Oriege@Campauleil & 0.964 & 0.954 & 0.902 & 0.972 & 0.966 & 0.898 & 0.975 & 0.963 & 0.890 \\
\hline 30 & Ouveze@Bedarrides & 0.862 & 0.959 & 0.957 & 0.880 & 0.959 & 0.947 & 0.868 & 0.961 & 0.932 \\
\hline 31 & Rizzanese@Barrage & 0.971 & 0.867 & 0.956 & 0.975 & 0.881 & 0.962 & 0.963 & 0.891 & 0.960 \\
\hline 32 & Romanche@Chambon & 0.949 & 0.991 & 0.896 & 0.957 & 0.992 & 0.892 & 0.956 & 0.987 & 0.894 \\
\hline 33 & Roya@Breil & 0.906 & 0.942 & 0.940 & 0.914 & 0.952 & 0.957 & 0.893 & 0.954 & 0.951 \\
\hline 34 & Salat@Roquefort & 0.993 & 0.925 & 0.900 & 0.991 & 0.919 & 0.923 & 0.993 & 0.916 & 0.926 \\
\hline 35 & Sioule@Fades & 0.954 & 0.987 & 0.895 & 0.962 & 0.984 & 0.891 & 0.962 & 0.987 & 0.901 \\
\hline 36 & Souloise@Infernet & 0.921 & 0.901 & 0.984 & 0.944 & 0.905 & 0.981 & 0.922 & 0.892 & 0.991 \\
\hline 37 & Stura@Lanzo & 0.969 & 0.903 & 0.881 & 0.986 & 0.908 & 0.899 & 0.986 & 0.884 & 0.907 \\
\hline 38 & Tarn@Cocures & 0.948 & 0.944 & 0.851 & 0.951 & 0.946 & 0.825 & 0.956 & 0.951 & 0.823 \\
\hline 39 & Tarn@Millau & 0.926 & 0.945 & 0.983 & 0.926 & 0.963 & 0.985 & 0.934 & 0.959 & 0.984 \\
\hline 40 & Tarn@Montbrun & 0.971 & 0.810 & 0.928 & 0.975 & 0.855 & 0.930 & 0.972 & 0.844 & 0.934 \\
\hline 41 & Tarn@Pinet & 0.930 & 0.936 & 0.916 & 0.929 & 0.925 & 0.920 & 0.919 & 0.931 & 0.914 \\
\hline 42 & Tarnon@Florac & 0.908 & 0.899 & 0.990 & 0.917 & 0.904 & 0.989 & 0.902 & 0.891 & 0.982 \\
\hline 43 & Taurion@RocheTalamie & 0.982 & 0.792 & 0.931 & 0.990 & 0.811 & 0.945 & 0.988 & 0.848 & 0.950 \\
\hline 44 & Tech@Reynes & 0.956 & 0.984 & 0.867 & 0.967 & 0.990 & 0.859 & 0.967 & 0.984 & 0.878 \\
\hline 45 & Tet@Vinca & 0.952 & 0.941 & 0.974 & 0.952 & 0.939 & 0.977 & 0.943 & 0.957 & 0.965 \\
\hline 46 & Tinee@PontLune & 0.988 & 0.868 & 0.954 & 0.988 & 0.839 & 0.962 & 0.992 & 0.881 & 0.962 \\
\hline 47 & Truyere@Grandval & 0.949 & 0.888 & 0.933 & 0.948 & 0.908 & 0.941 & 0.950 & 0.920 & 0.939 \\
\hline 48 & Ubaye@RocheRousse & 0.923 & 0.886 & 0.980 & 0.914 & 0.930 & 0.986 & 0.904 & 0.928 & 0.988 \\
\hline 49 & Vence@Francheville & 0.991 & 0.787 & 0.926 & 0.985 & 0.803 & 0.934 & 0.994 & 0.799 & 0.931 \\
\hline 50 & Vienne@Bussy & 0.934 & 0.944 & 0.906 & 0.943 & 0.949 & 0.920 & 0.950 & 0.946 & 0.922 \\
\hline
\end{tabular}




\section{Agout@Fraisse}

Year 1986

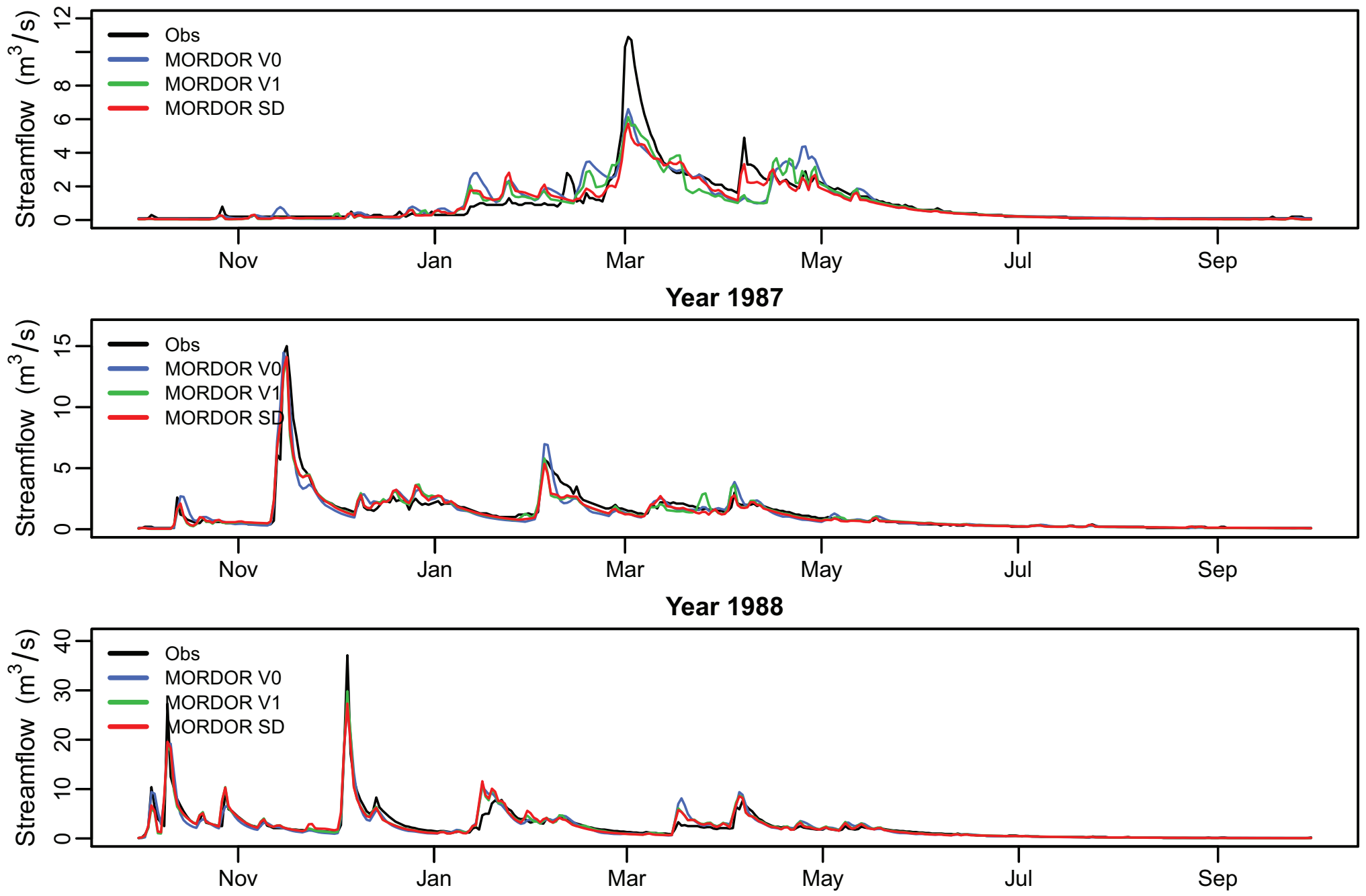


Allier@Poutes

Year 1989

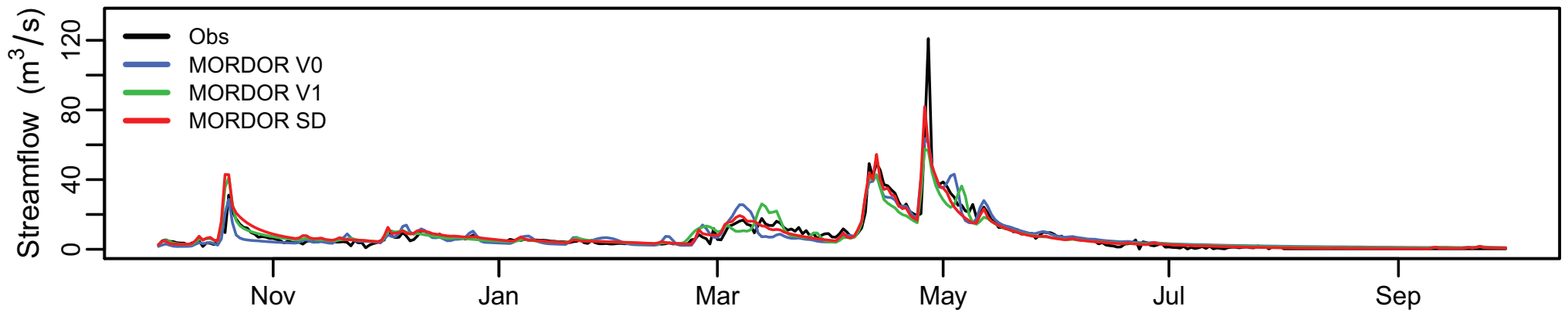

Year 1990
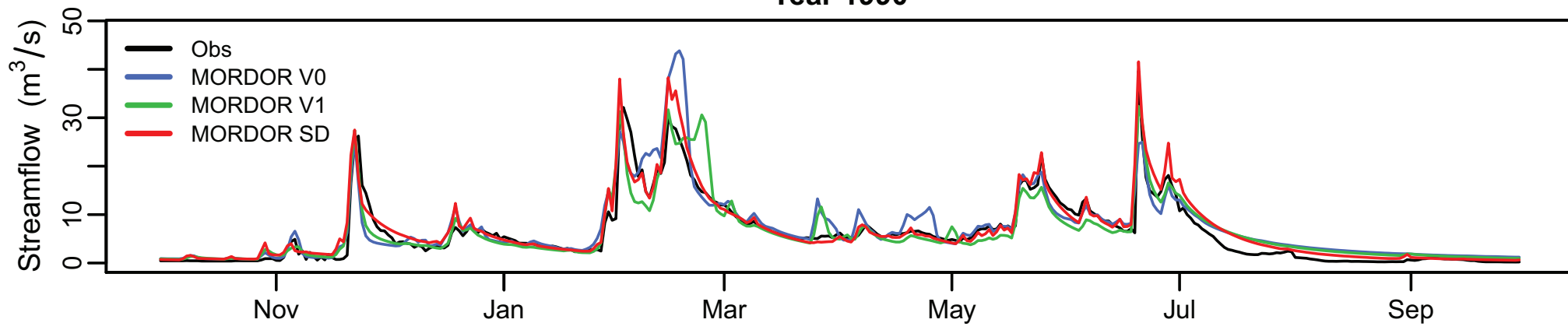

Year 1991
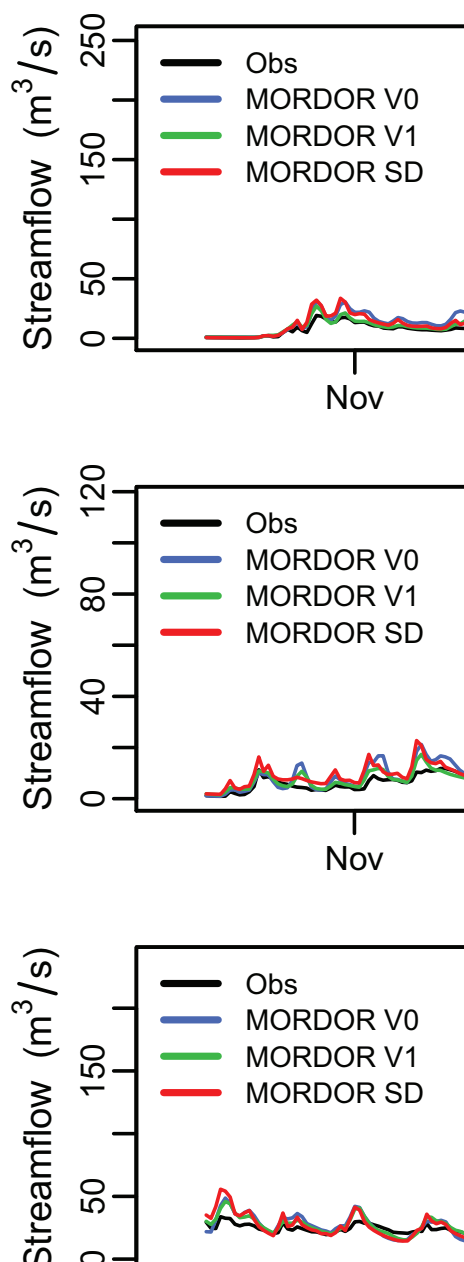

$\longrightarrow$ MORDOR V1

$\longrightarrow$ MORDOR SD
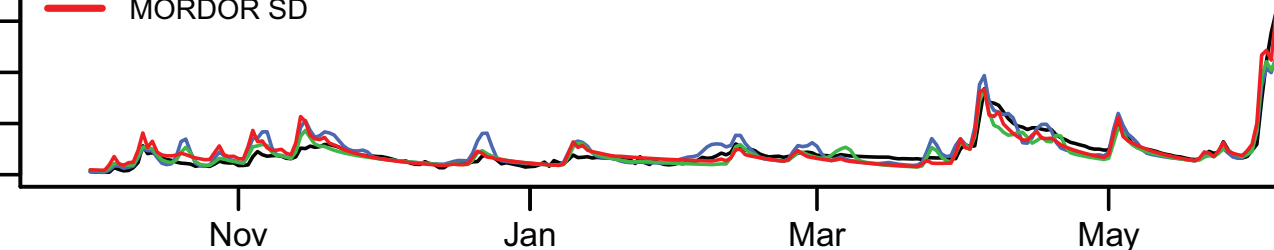

Year 1992
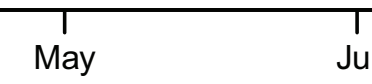

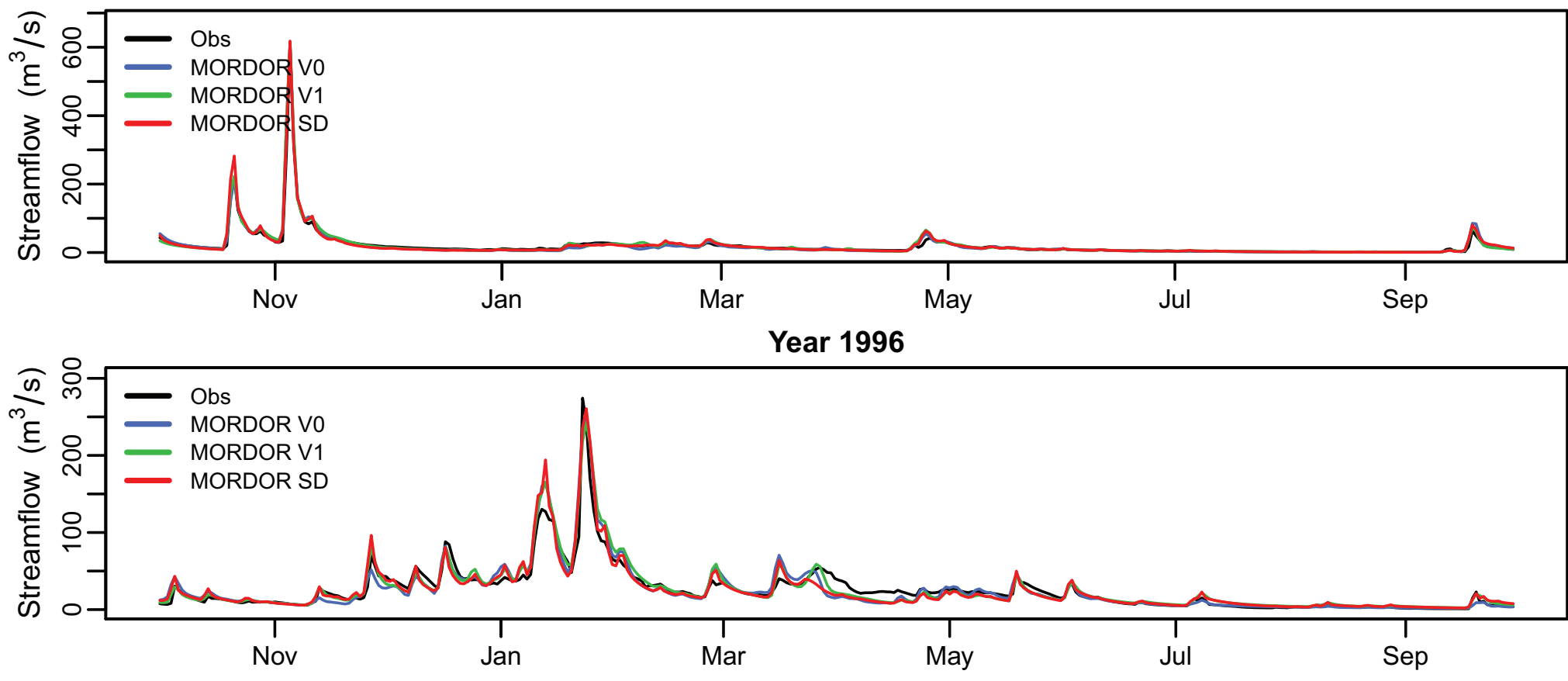

Year 1996

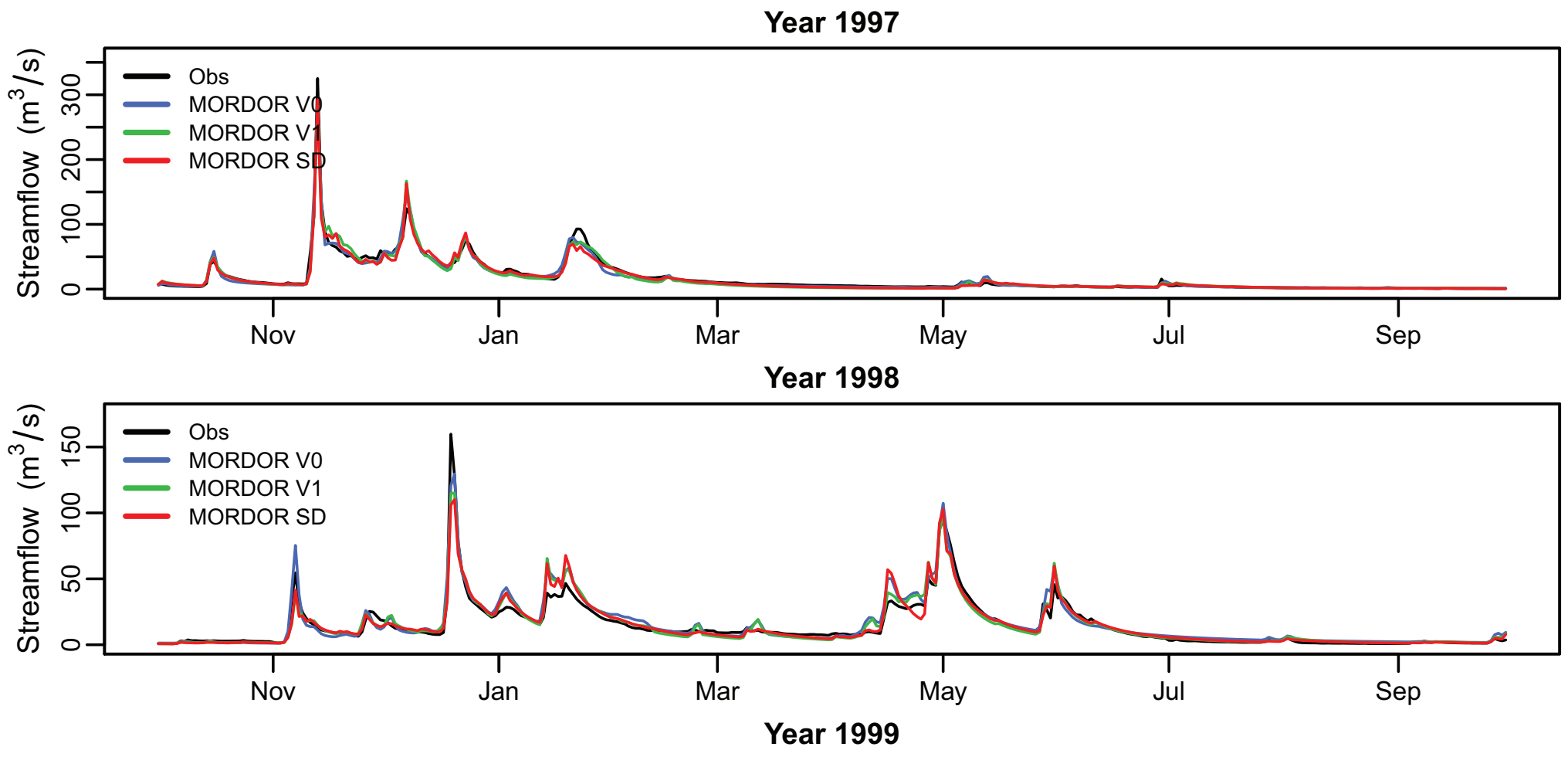

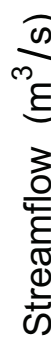




\section{Ardeche@Sauze}

Year 1974

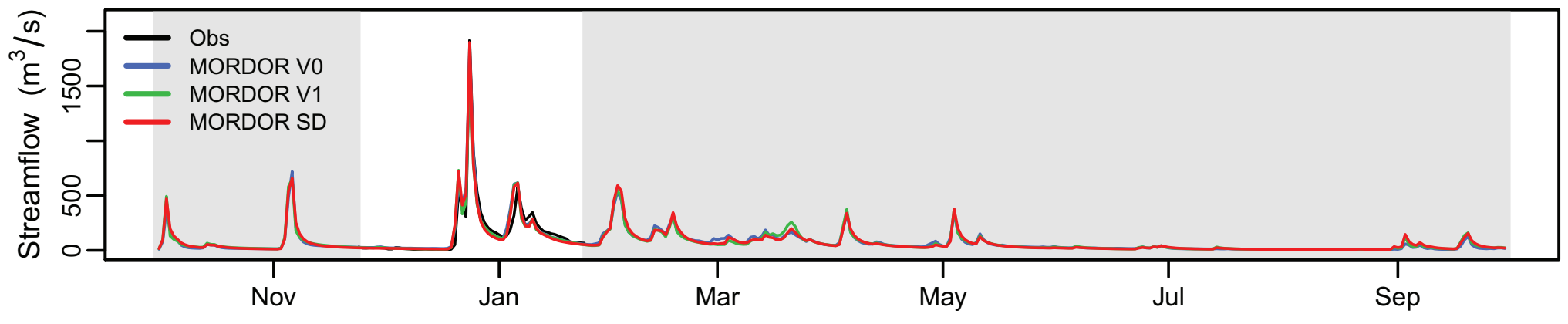

Year 1975
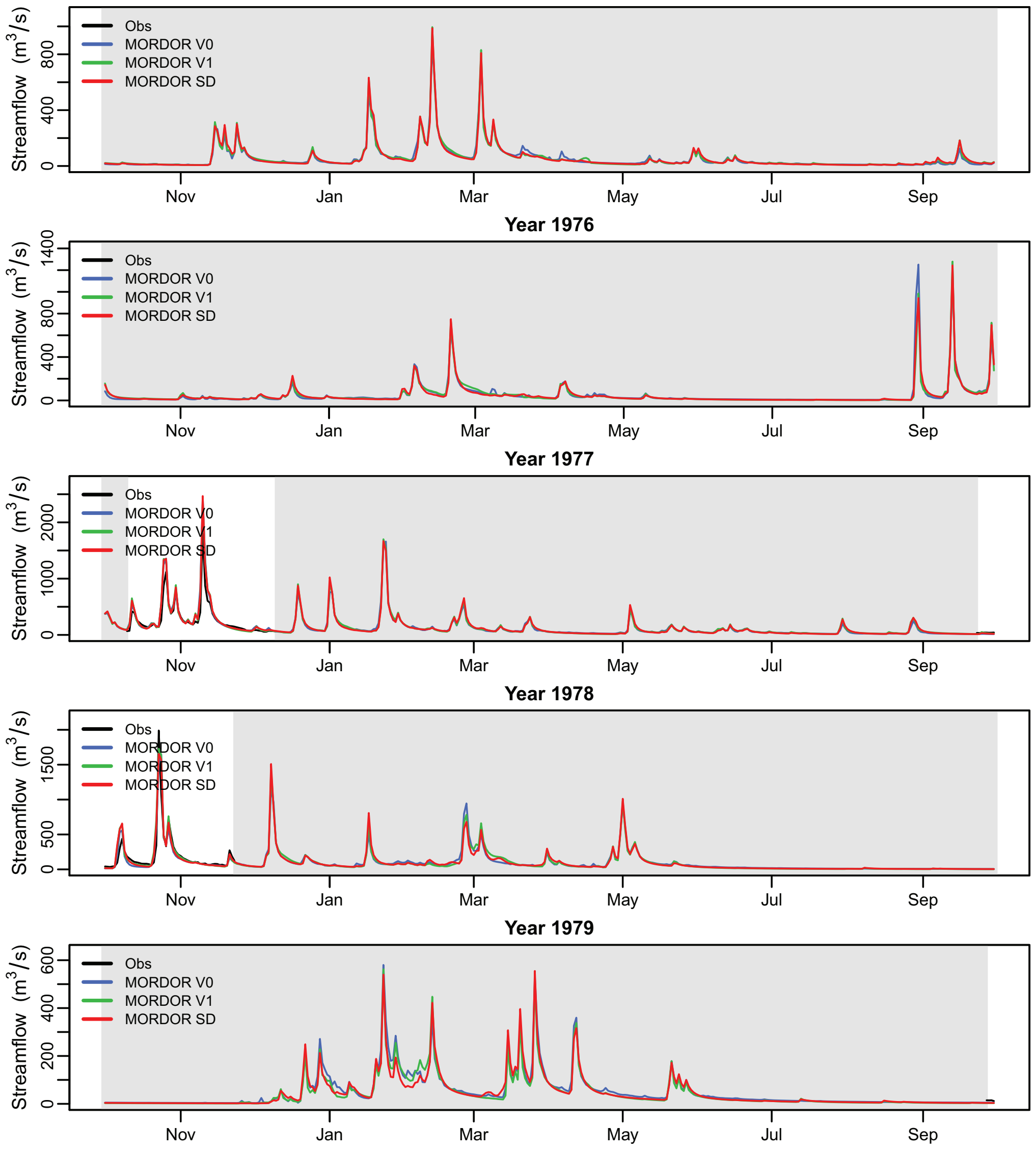


\section{Arn@Taillades}

\section{Year 1988}

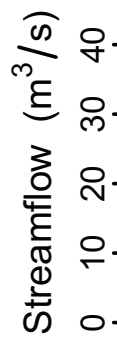

三
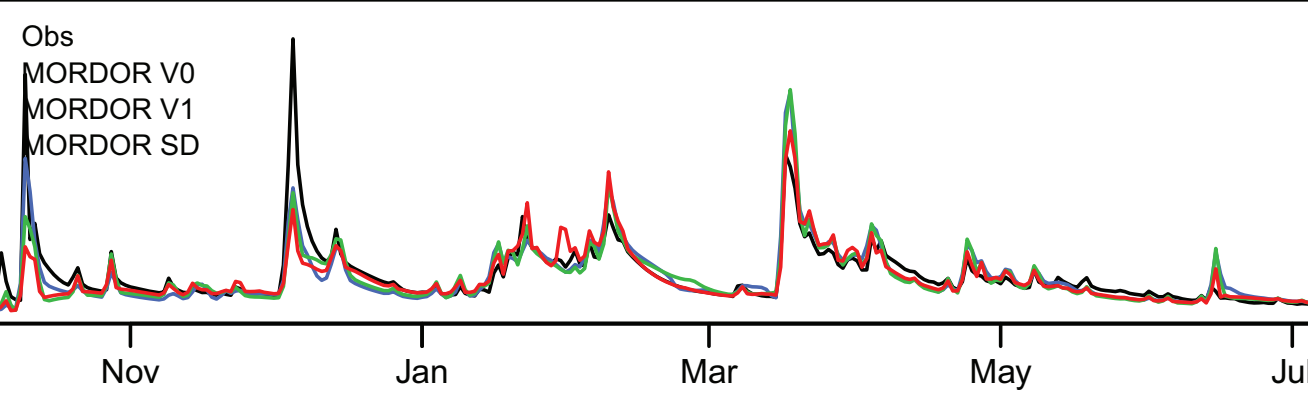

Year 1989
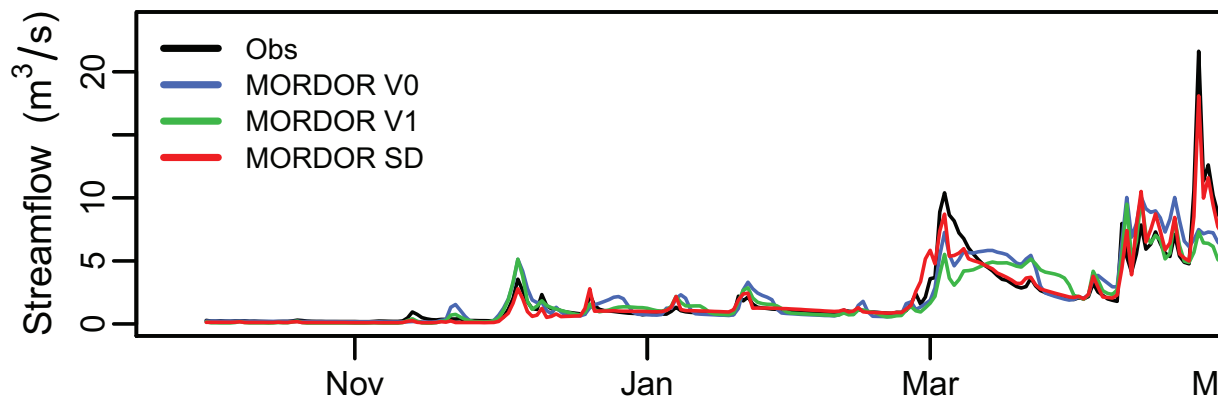

Year 1990
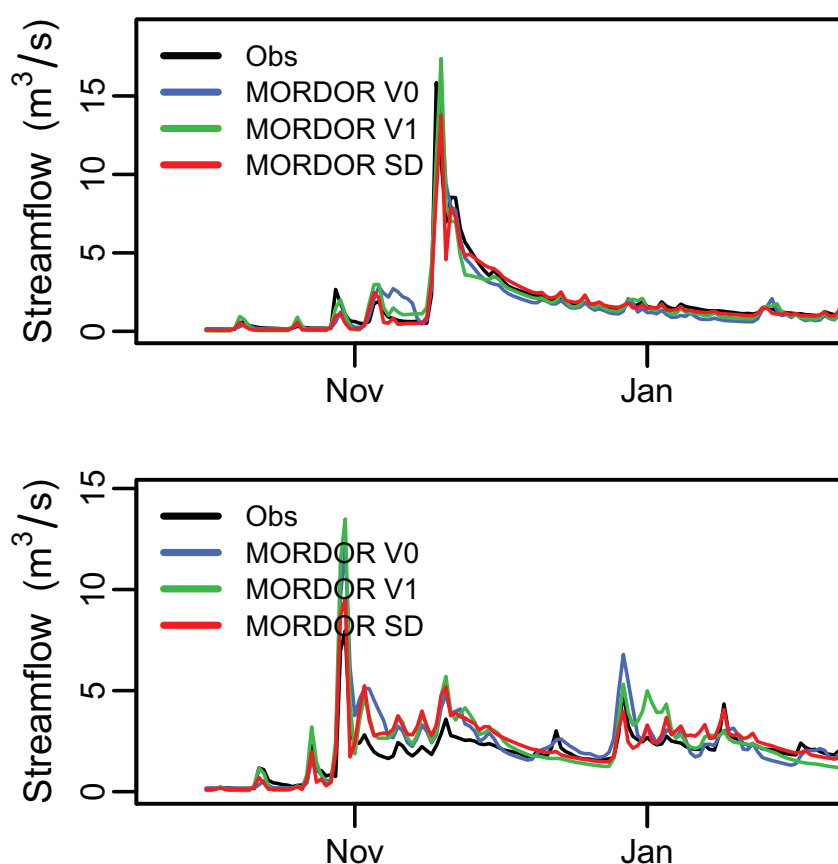

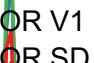

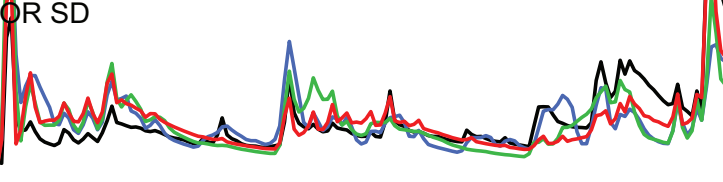

Nov
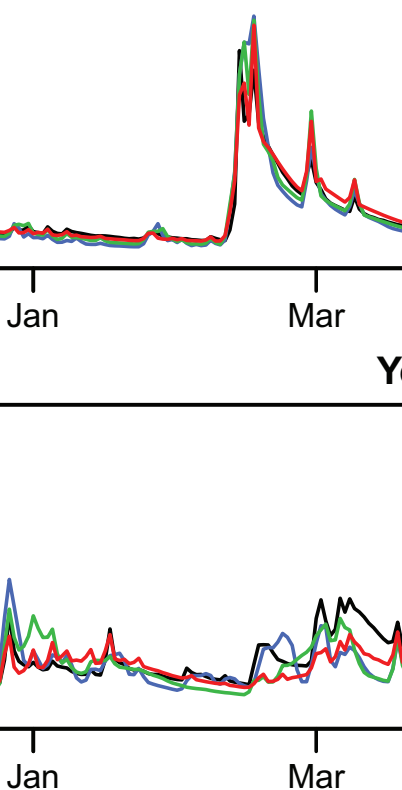

Year 1991

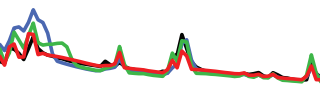

May

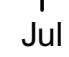

Year 1991
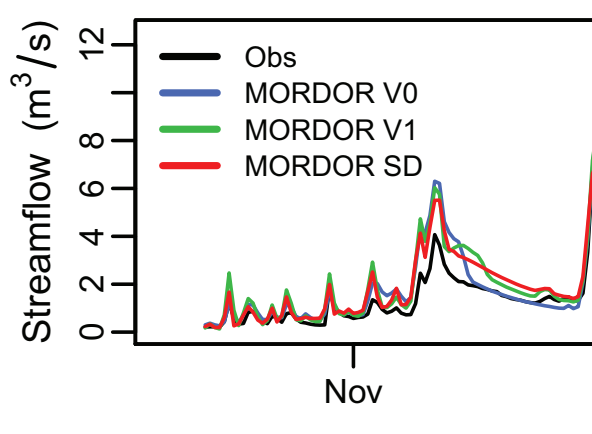

Year 1992

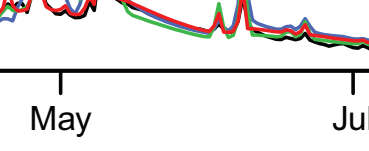

AM
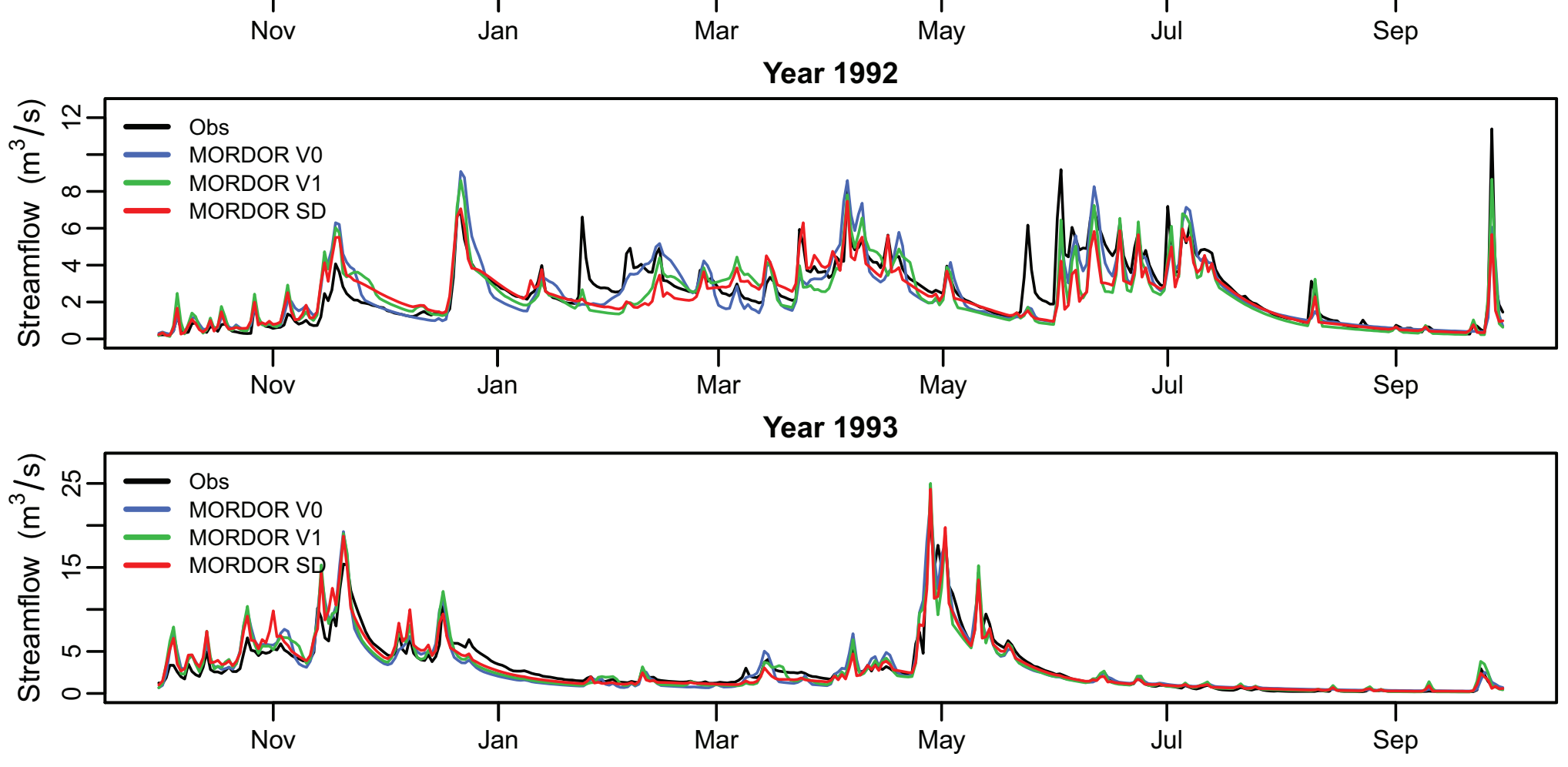


\section{Arn@Taillades}

\section{Year 1994}

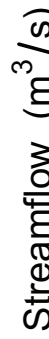

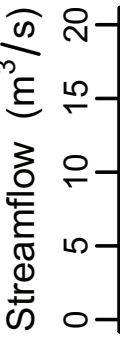

$\longrightarrow$ Obs

- MORDOR VO

- MORDOR V1

으- MORDORSD

1.

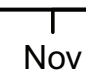

Jan
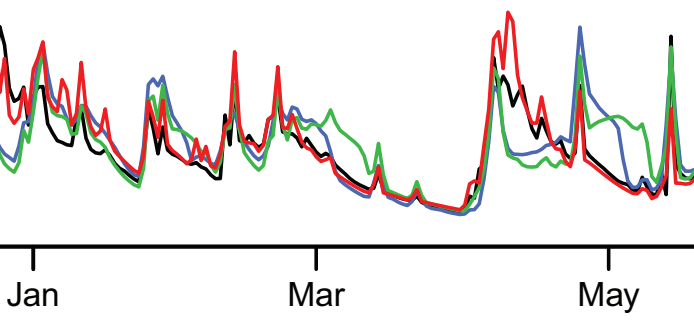

Year 1995
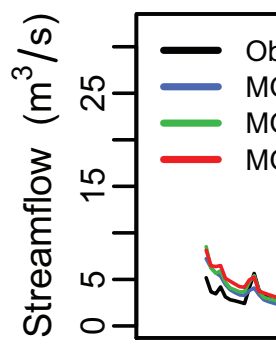

Obs

bo

V1

SD

Me bundon the

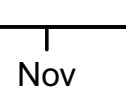

Jan

Mar

Year 1996

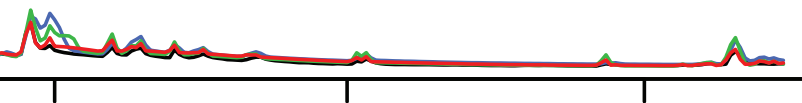

May

Jul

Sep

क
m
है
3
0
0
का
0
0
0

Obs

- - MORDOR V0

그 MORDOR SD

ㄱ-

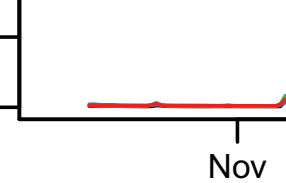

Nov

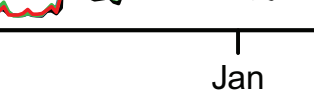

Jan

rov

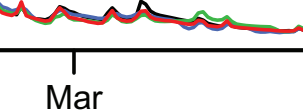

Year 1997
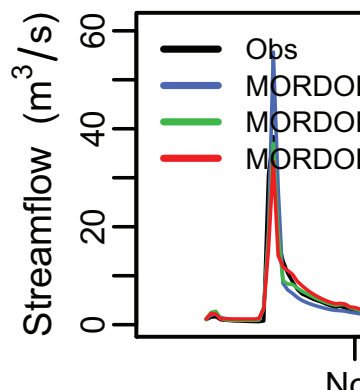

Nov
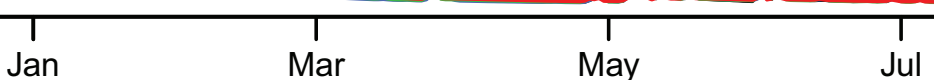

Year 1998
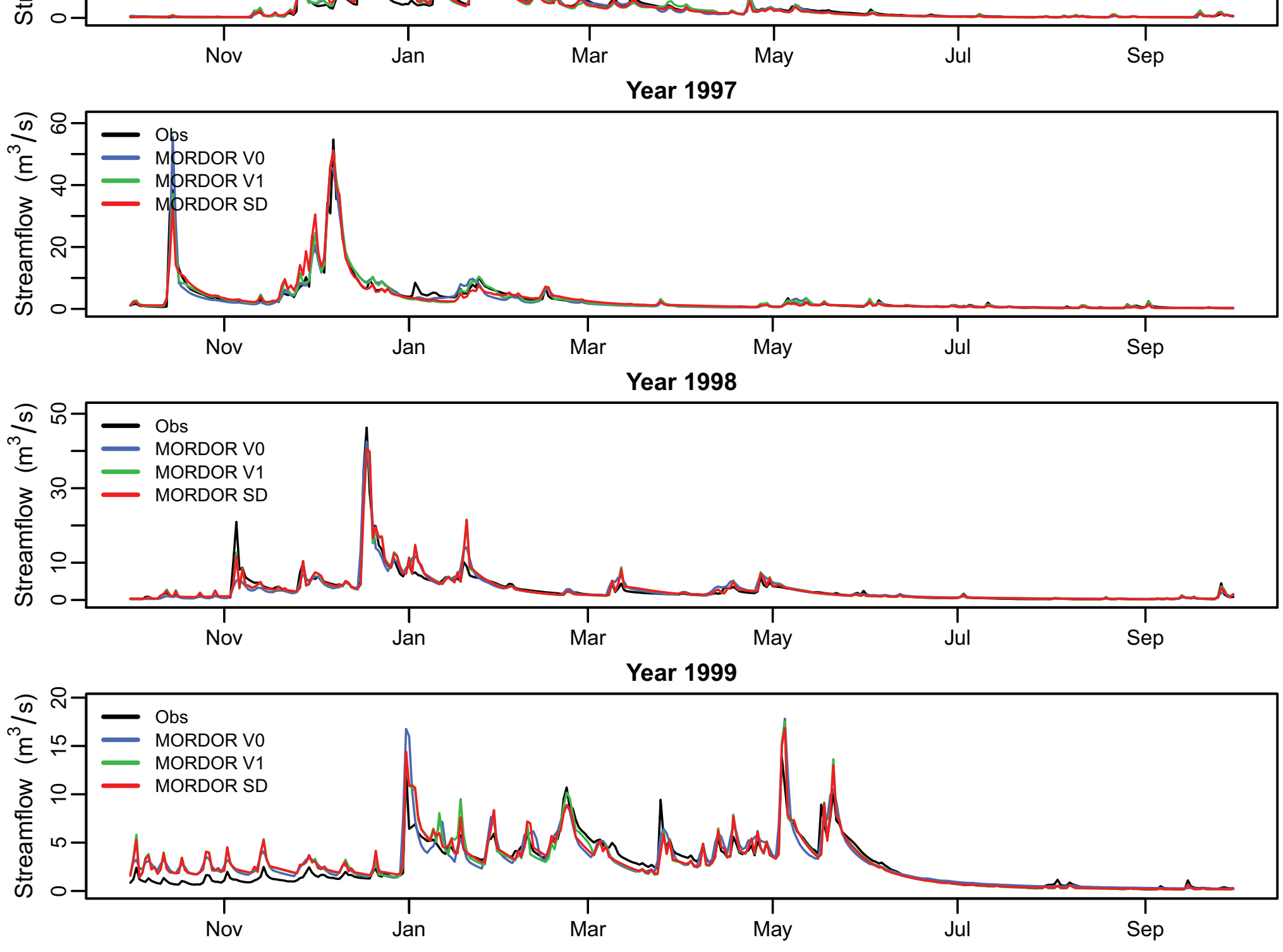


\section{Breze@Meyrueis}

\section{Year 1986}

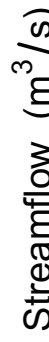

8

$\longrightarrow$ Obs

MORDOR VO

MORDOR V1

MORDOR SD

Nov
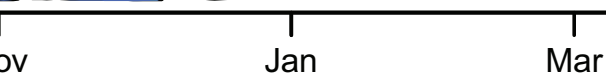

$\therefore$.

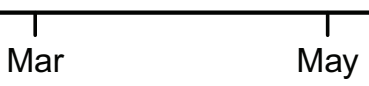

Year 1987
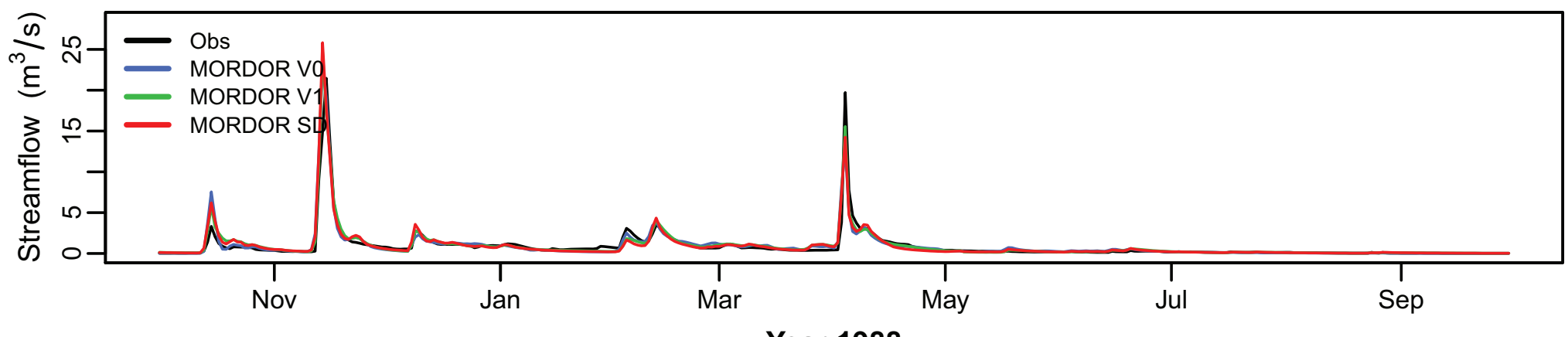

Year 1988

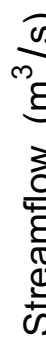

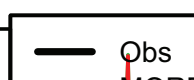




\section{Breze@Meyrueis}

Year 1992

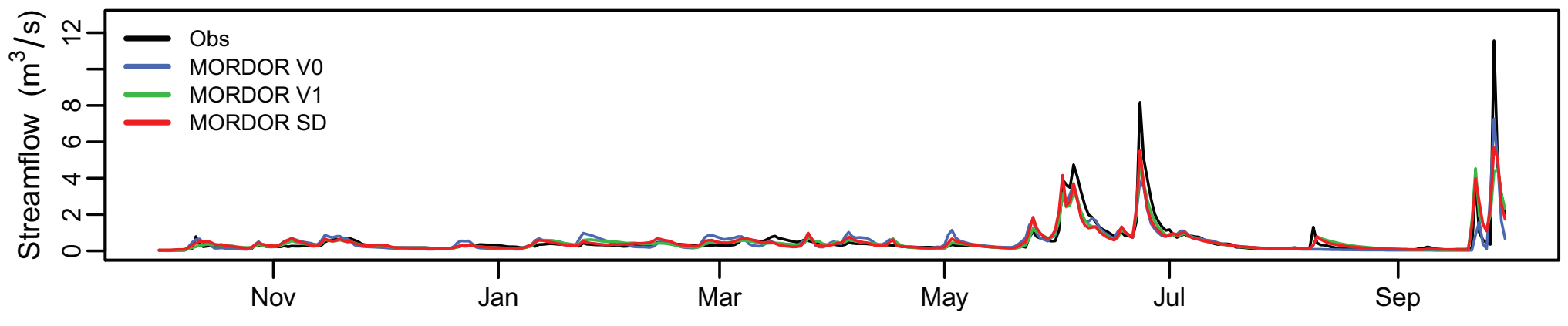

Year 1993
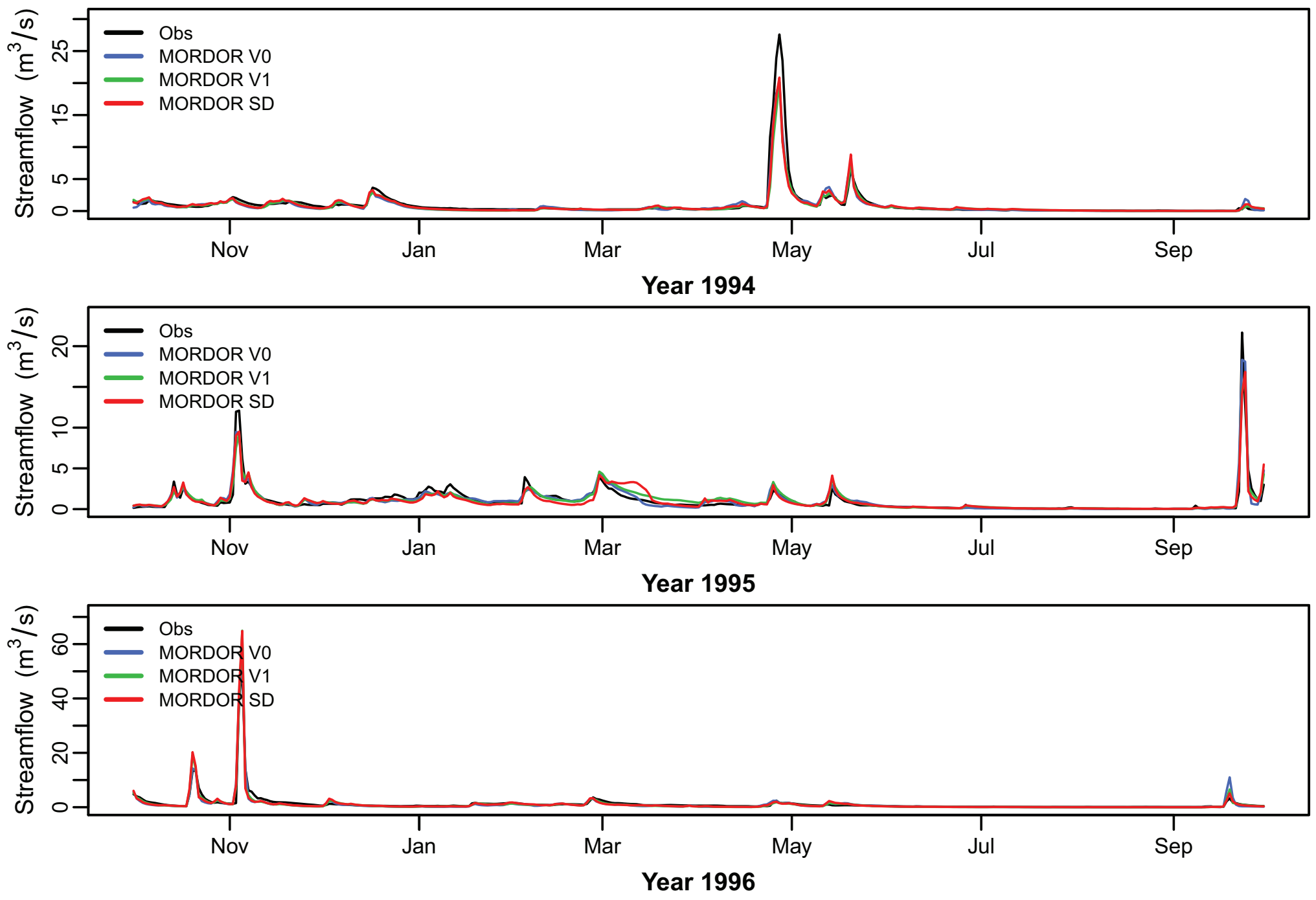

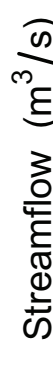

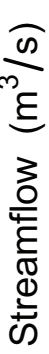


Bromme@Brommat

Year 1990

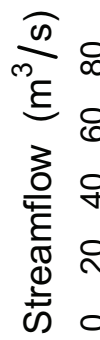

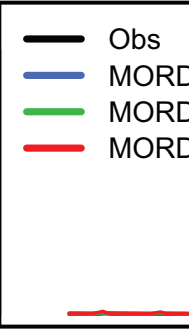

Obs

MORDOR V0

Nov

han ans

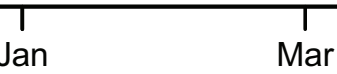

Mar May

Year 1991

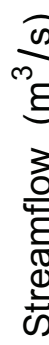

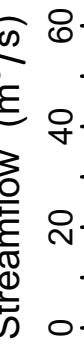

$-7$

Obs

MORD申R V0

MORD R V1

MORD R SD
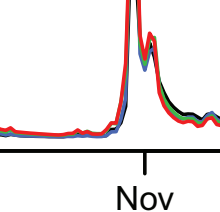

Nov

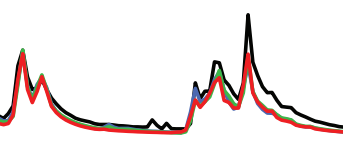

Mar

Year 1992

$\frac{\pi}{m}$
$\xi$
$\xi$
3
5
0
0
0

- Obs

- MORDOR V0

- MORDOR Sq

ㅇ-

- an

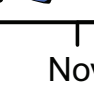

N

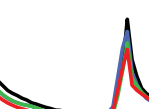

1

Jan

Mar

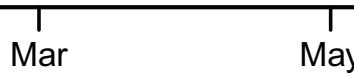

Year 1993
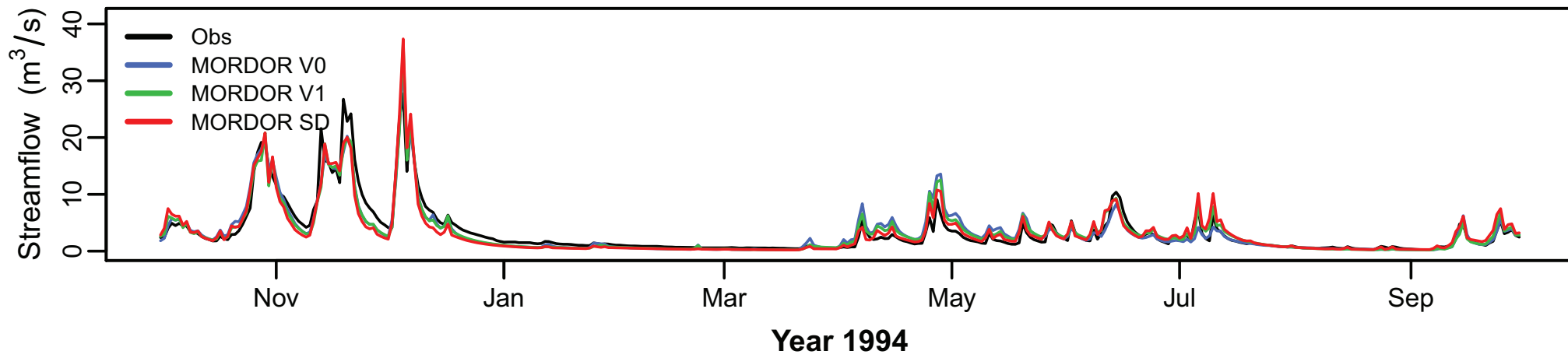

Year 1994

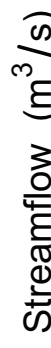

Obs

- MORDOR VO

MORDOR V1

MORDOR SD

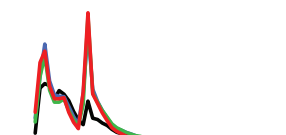

Nov
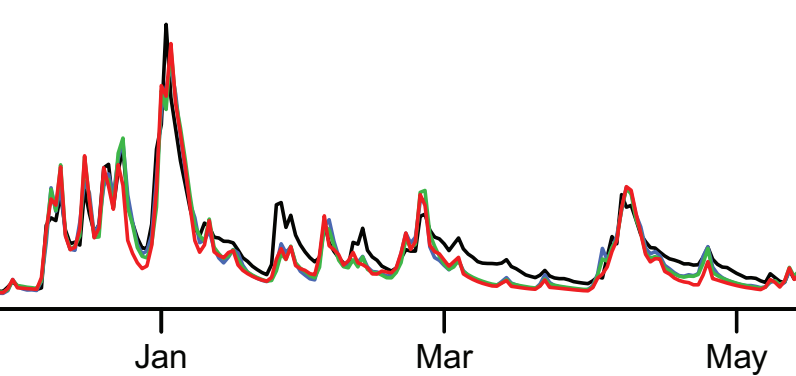

M

n

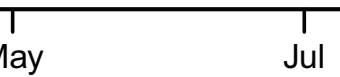

Sep

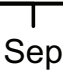

L 
Bromme@Brommat

Year 1996

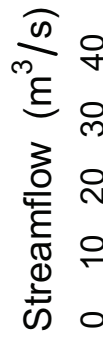

三

Obs

MORDOR V0

MORDOR V1

- MORDOR SD

dentans
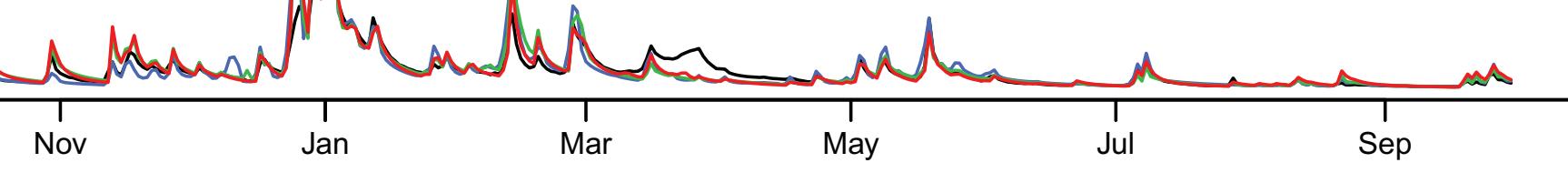

Year 1997

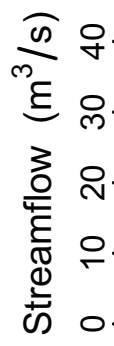

- Obs

- MORDOR V0

MORDOR V1

ㅇ--

- MORDOR SD

co

$\begin{array}{ll} & =\text { Obs } \\ \text { MORDOR V0 } & \text { MORDOR V1 } \\ \text { MORDOR SD } & \text { MORDOR }\end{array}$

Year 1998

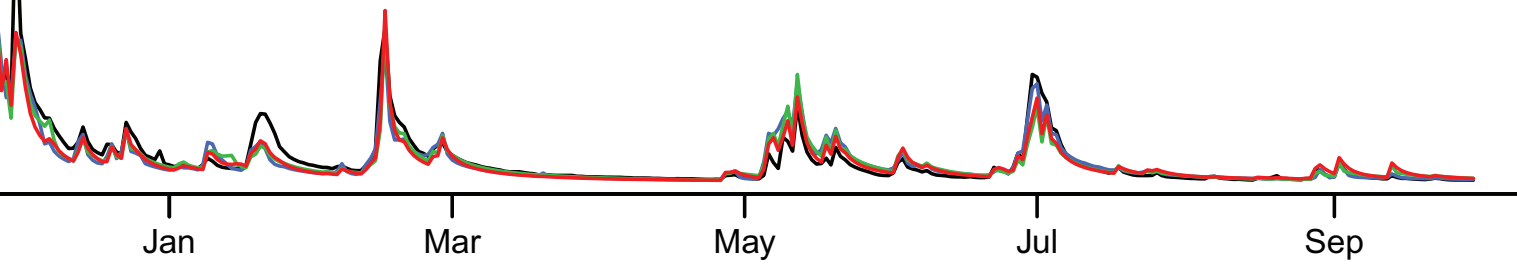

Year 1998 


\section{Ceze@Bagnols}

Year 1995
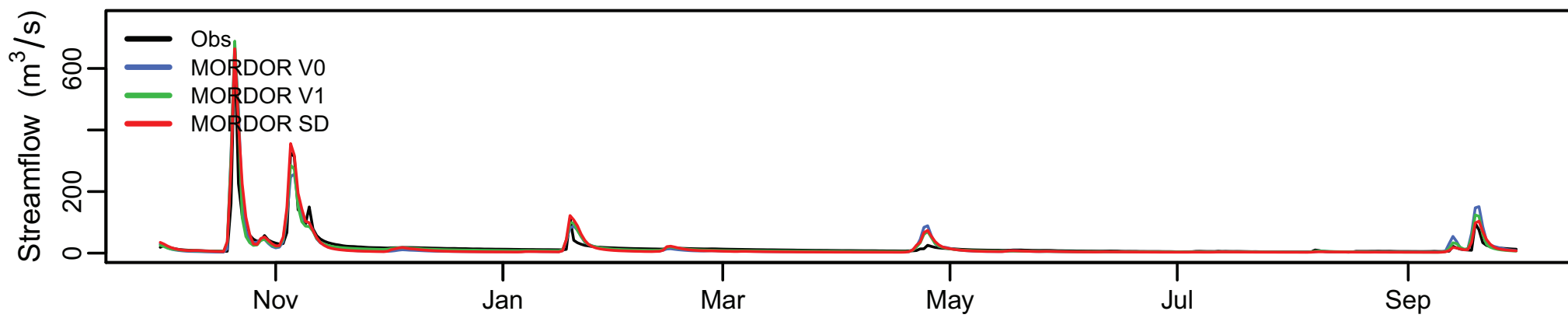

Year 1996
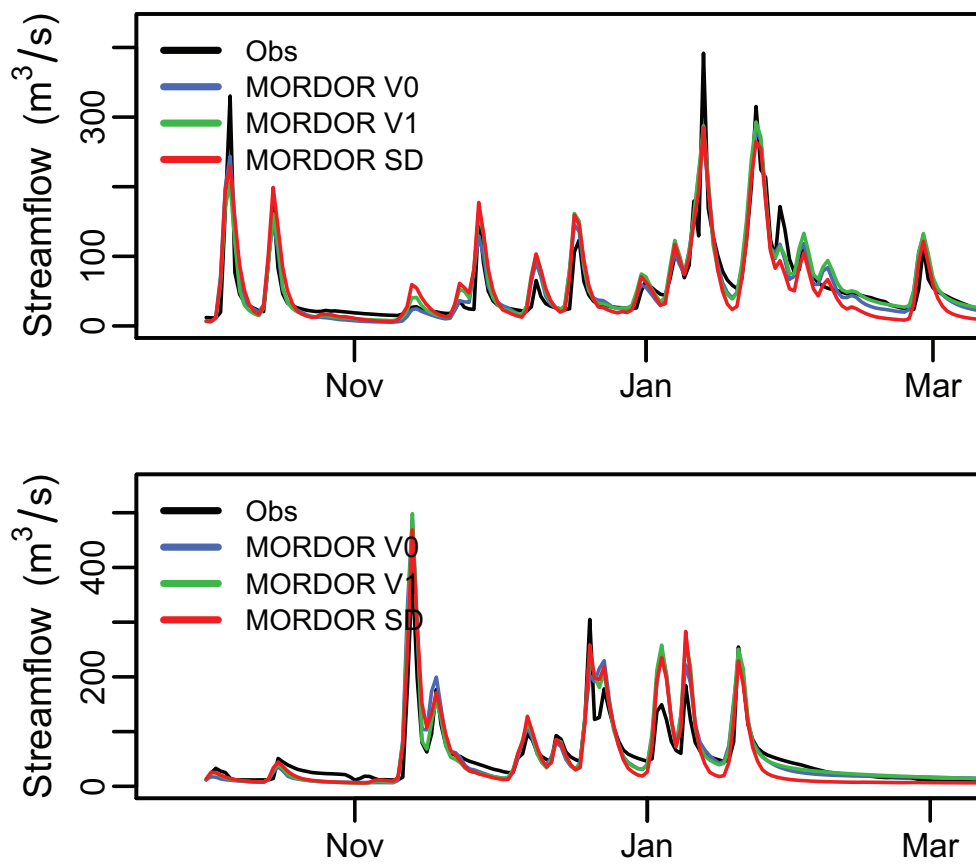

Year 1998
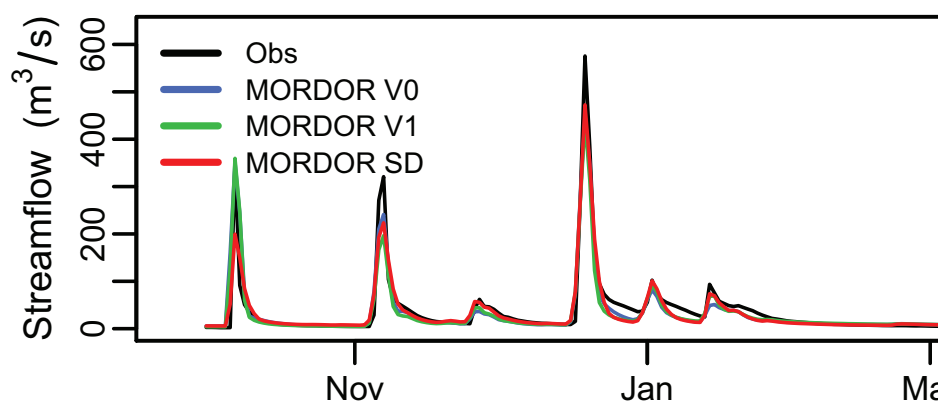

Year 1997

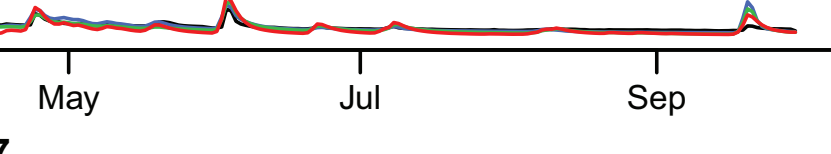

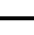




\section{Chassezac@SteMarguerite}

Year 1987

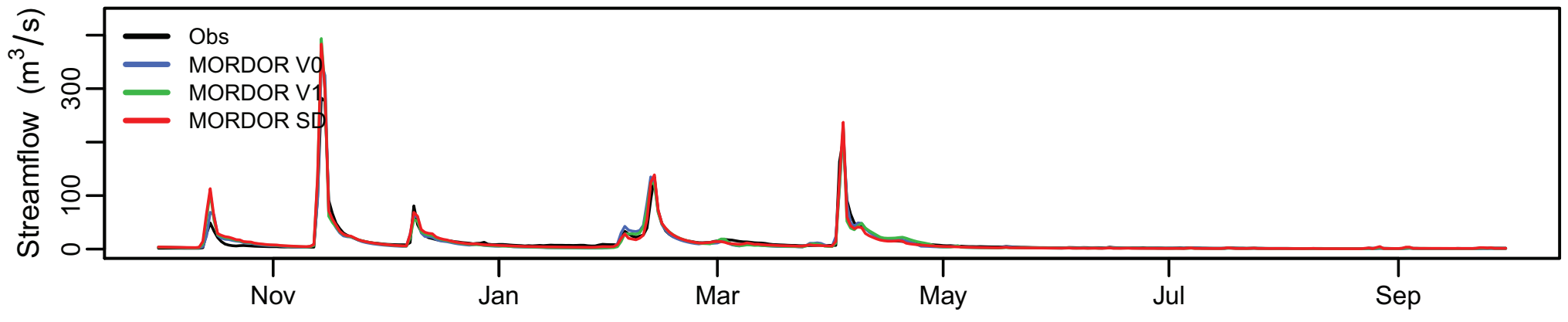

Year 1988
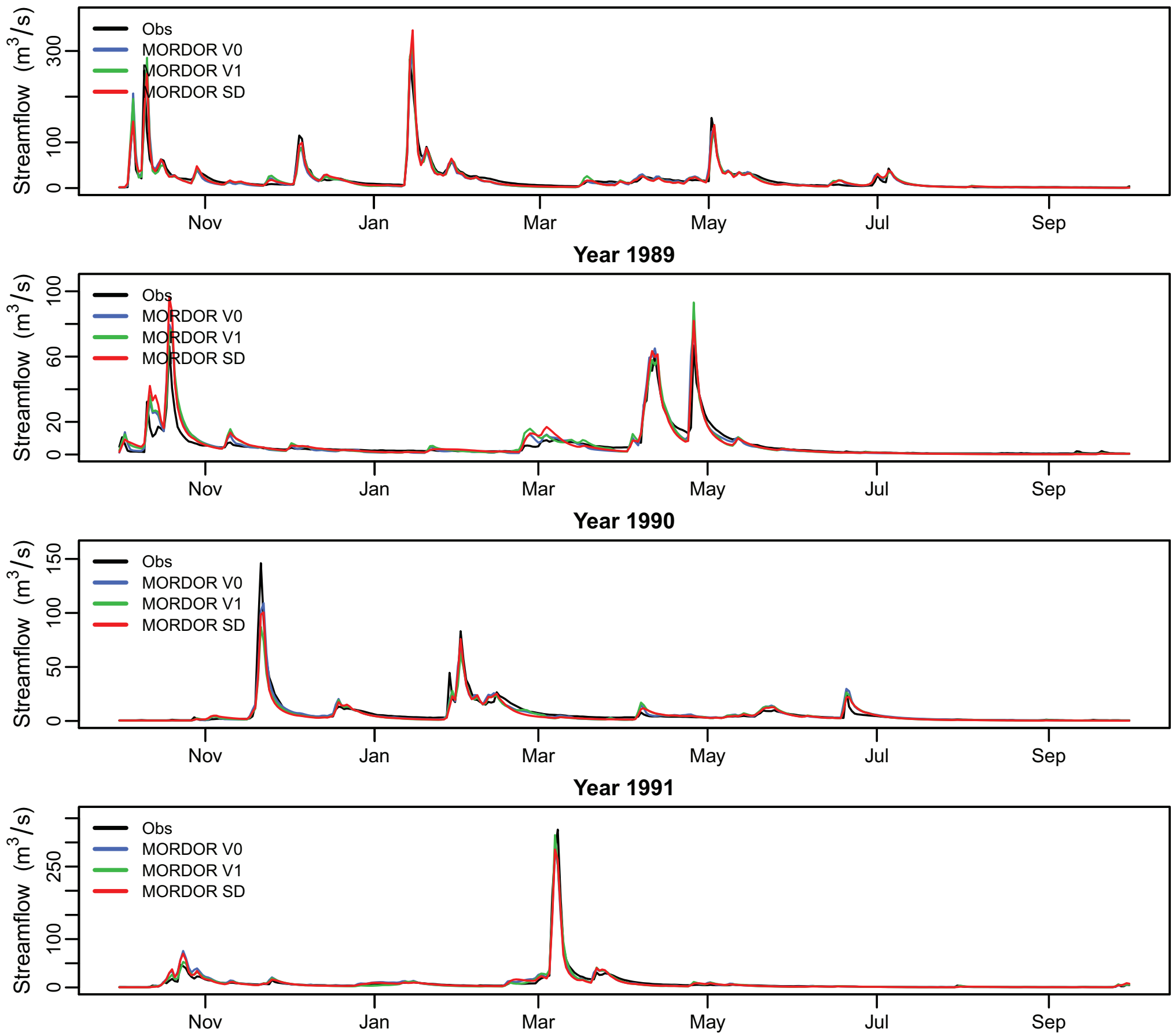

Year 1992

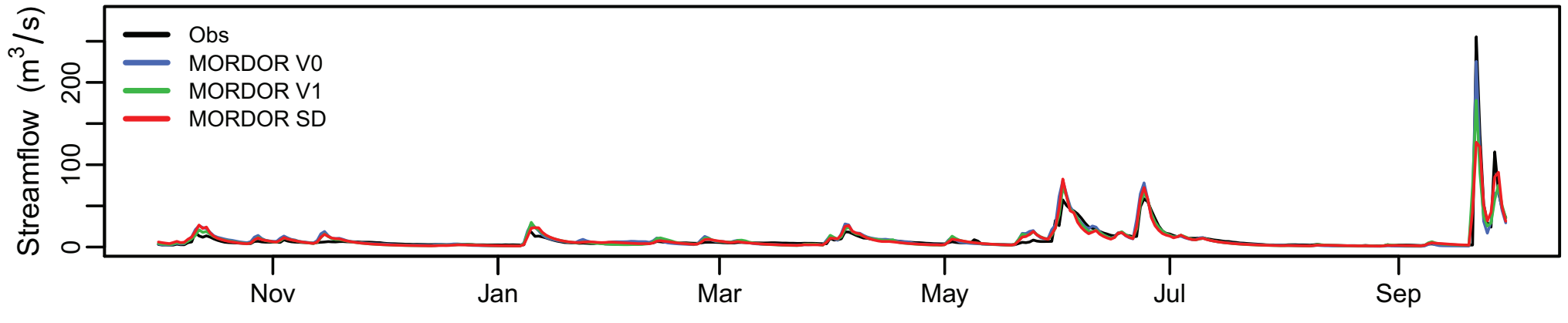


Chassezac@SteMarguerite

Year 2005

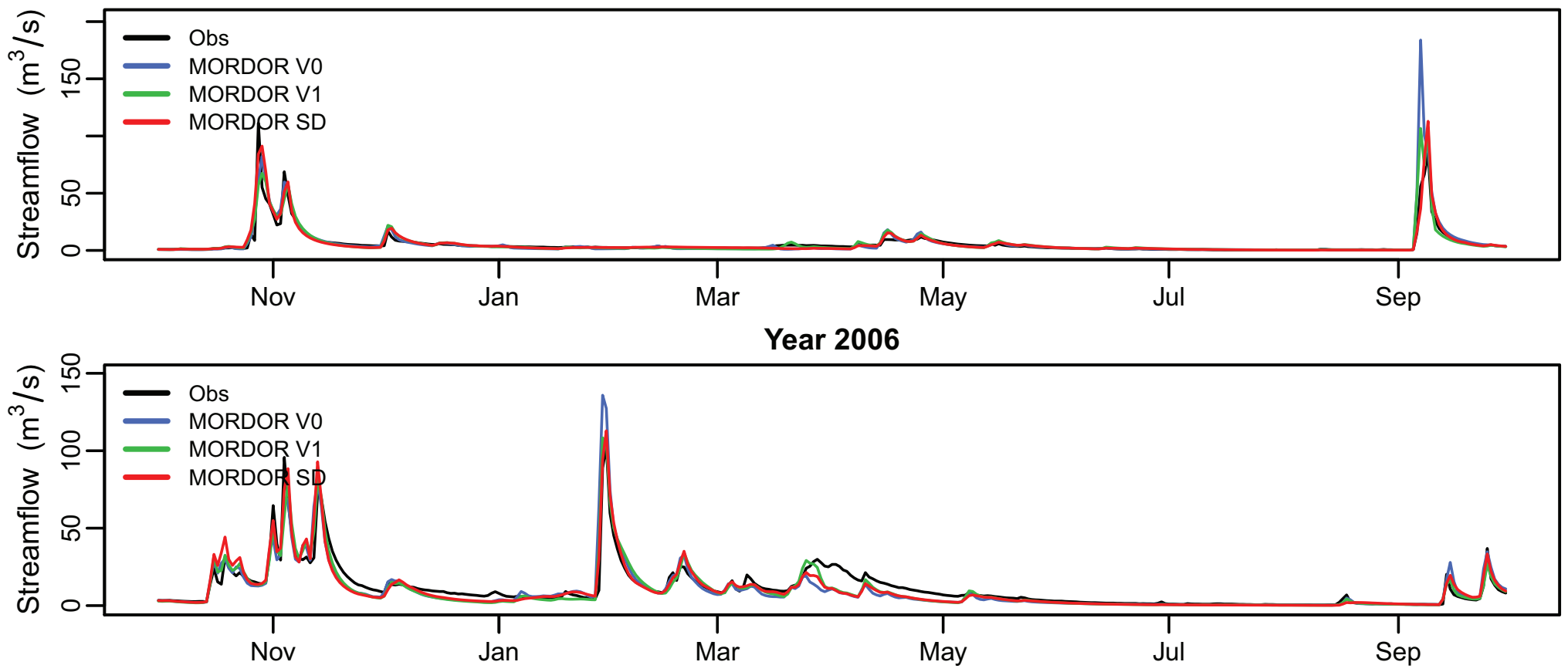




\section{Creuse@Age}

Year 1979
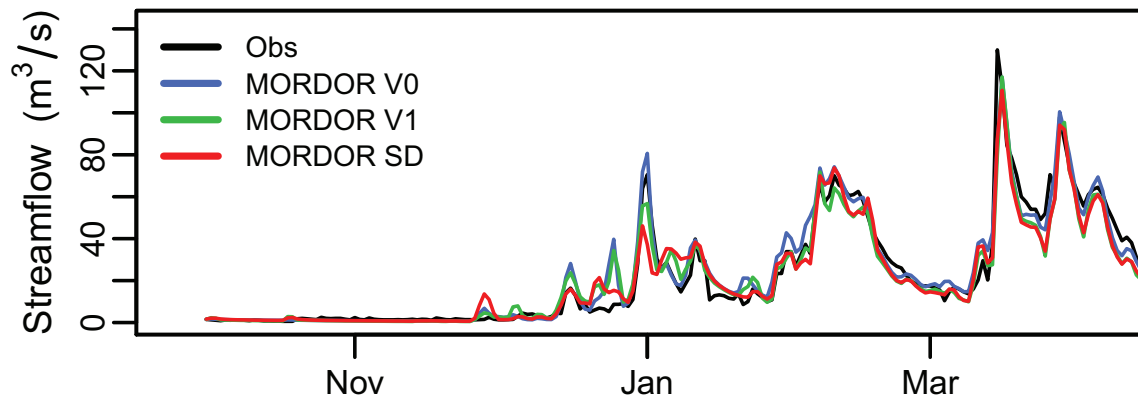

Year 1980
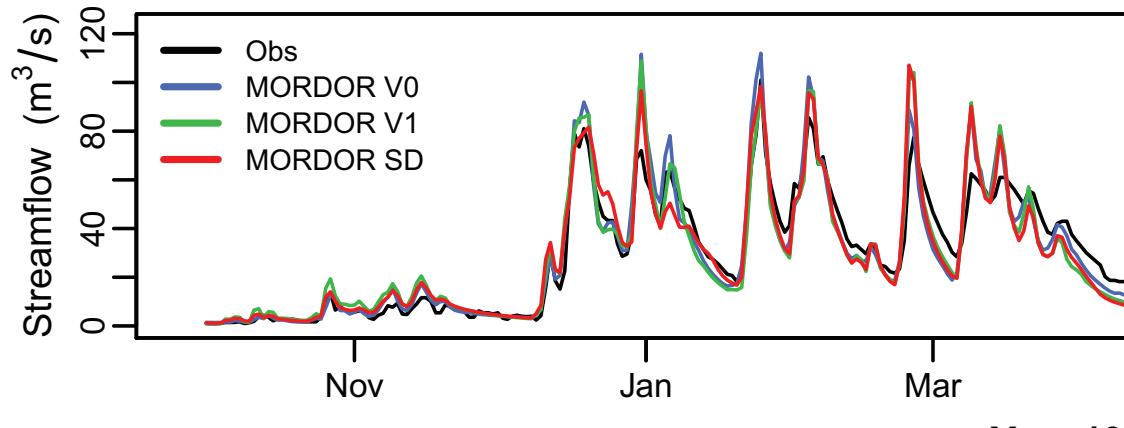

Year 1981

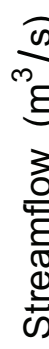

- Obs

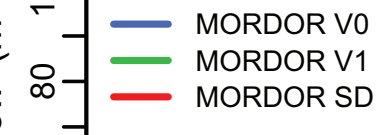

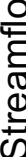

웅

nad d

Nov

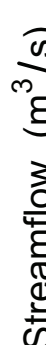

क
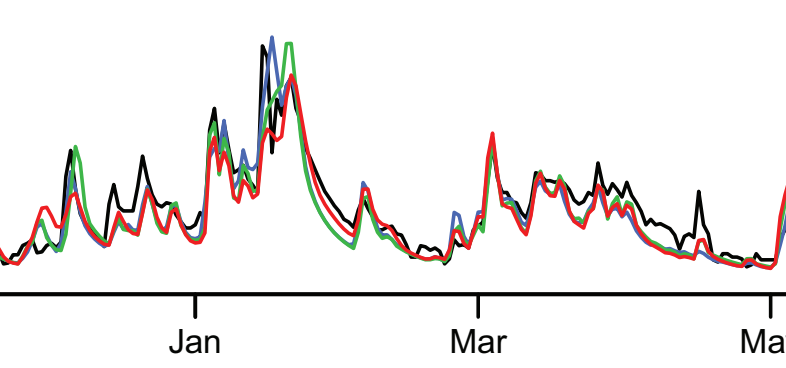

11

Jan

Year 1982

May

Mrand

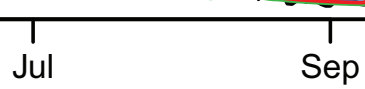

Sep
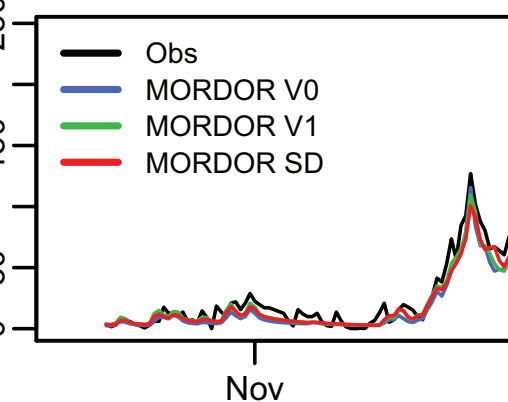

anl

brenande

Jan

Mar

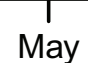

Jul

Sep

Year 1983

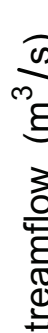
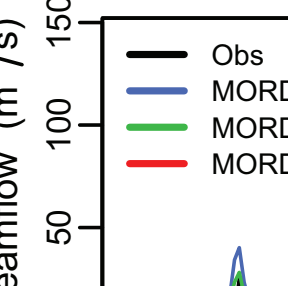

MORDOR V0

MORDOR V1
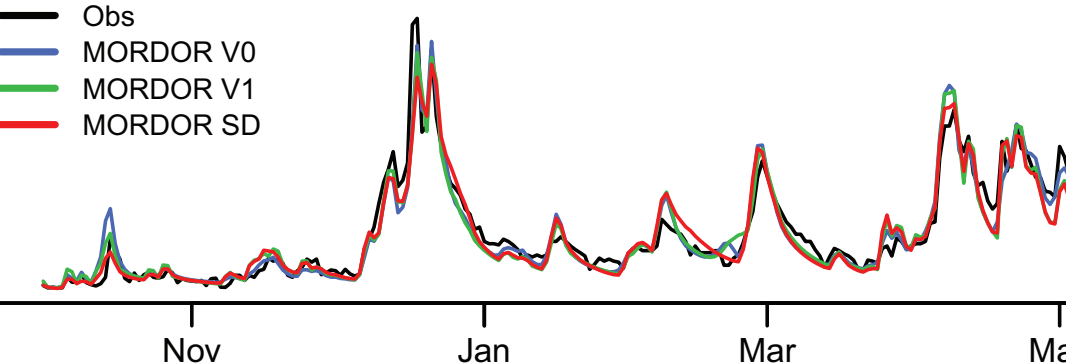

Year 1984

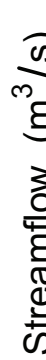

Nov

an

Year 1984

ㄱ- Obs

O- MORDOR V0

- MORDOR V1

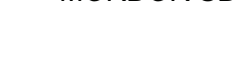

Nom
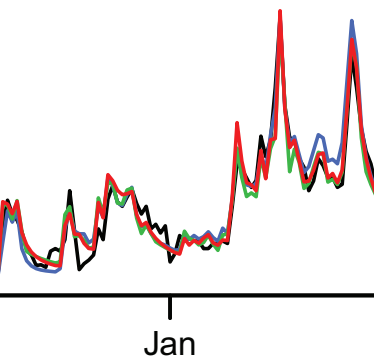

Mar du

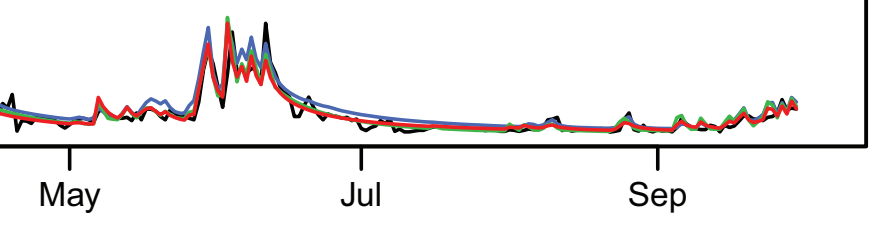




\section{Creuse@Age}

Year 1985

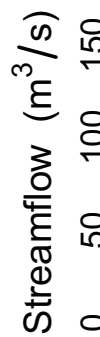

$\begin{array}{ll}- & \text { Obs } \\ - & \text { MORDOR V0 } \\ \text { MORDOR V1 }\end{array}$

— MORDOR SD

으-1

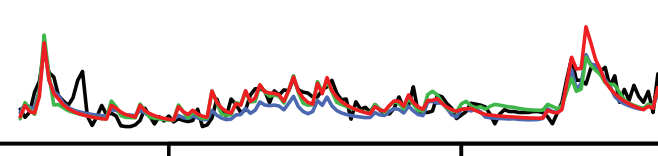
Nov
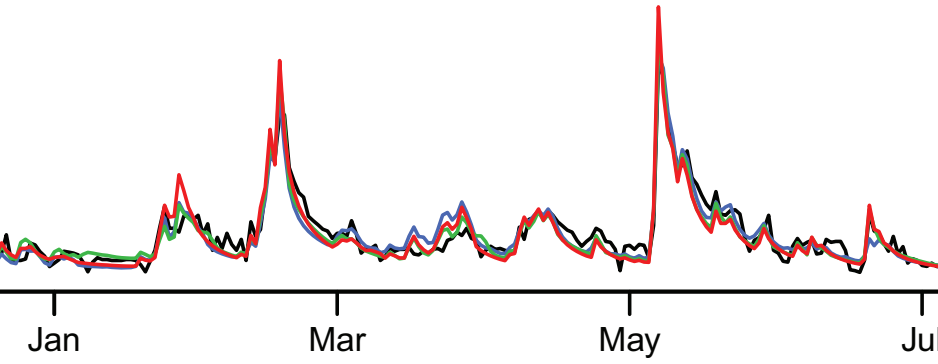

Jul Sep

Year 1986
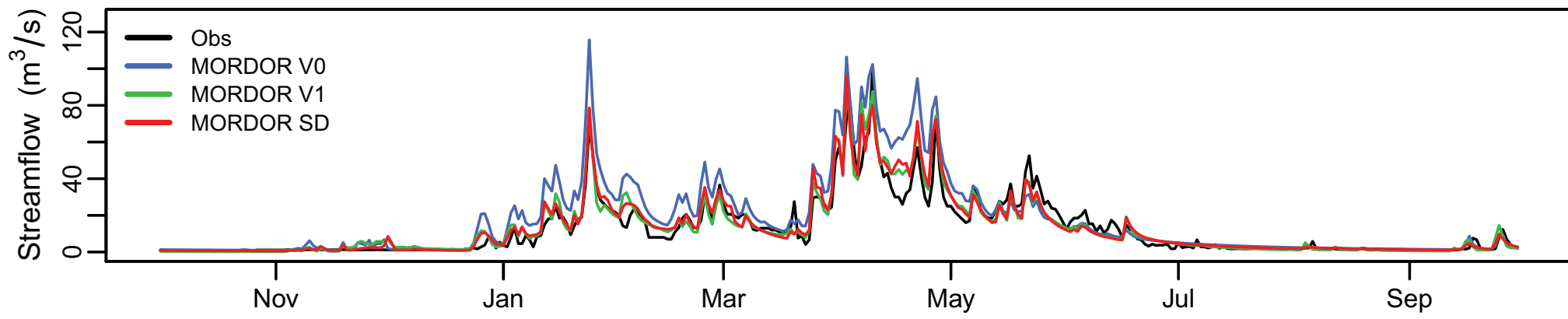

Year 1987

$\frac{0}{0}$
m
3
0
$\frac{0}{4}$
$\frac{\pi}{0}$
0
0

- Obs

- MORDOR V1

MORDOR SD

- Nunsurdenth

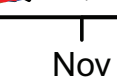

Jan

an Mar

Year 1988

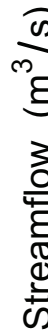

$\longrightarrow$ Obs

MORDOR V0 $=\begin{aligned} & \text { MORDOR V1 } \\ & \text { MORDOR SD }\end{aligned}$

MORDOR V1 $\begin{aligned} & \text { MORDOR V0 } \\ & \text { MORDOR SD }\end{aligned}$

MORDOR V1 $\begin{aligned} & \text { MORDOR V0 } \\ & \text { MORDOR SD }\end{aligned}$

Nov

Jan Mar

Year 1989

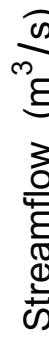

- Obs

ㄱ- $=\begin{aligned} & \text { Obs } \\ & \text { MORDOR V0 } \\ & \text { MORDOR V1 } \\ & \text { MORDOR SD }\end{aligned}$

है

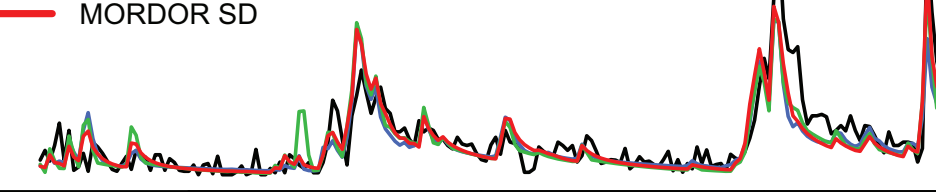

Nov

Jan

Mar

Year 1990
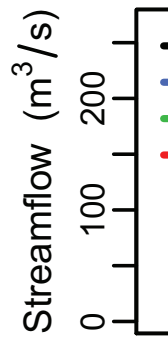

Obs

- MORDOR V0

- MORDOR V1

$\longrightarrow$ MORDOR SD

음

Nov

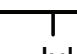

Jul 


\section{Creuse@Age}

Year 1997
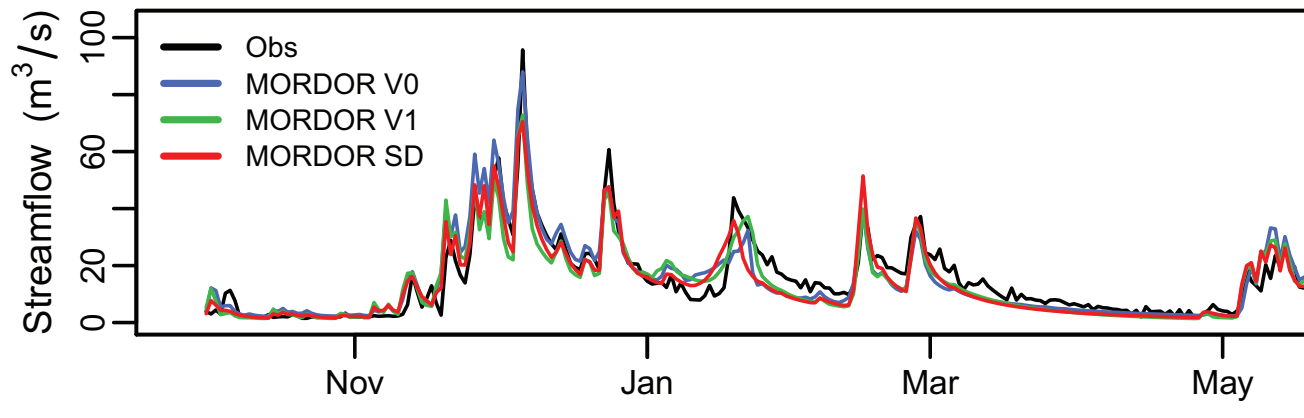

Year 1998
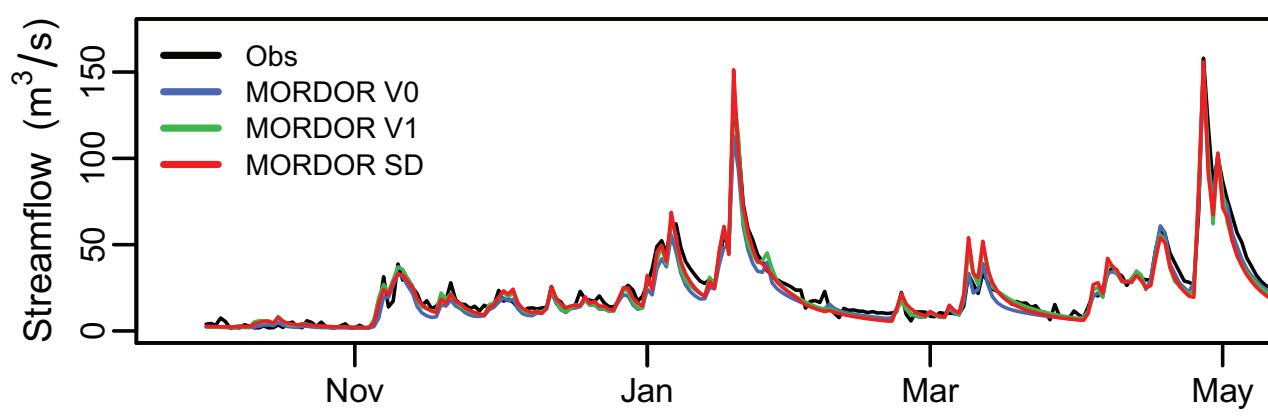

Year 1999
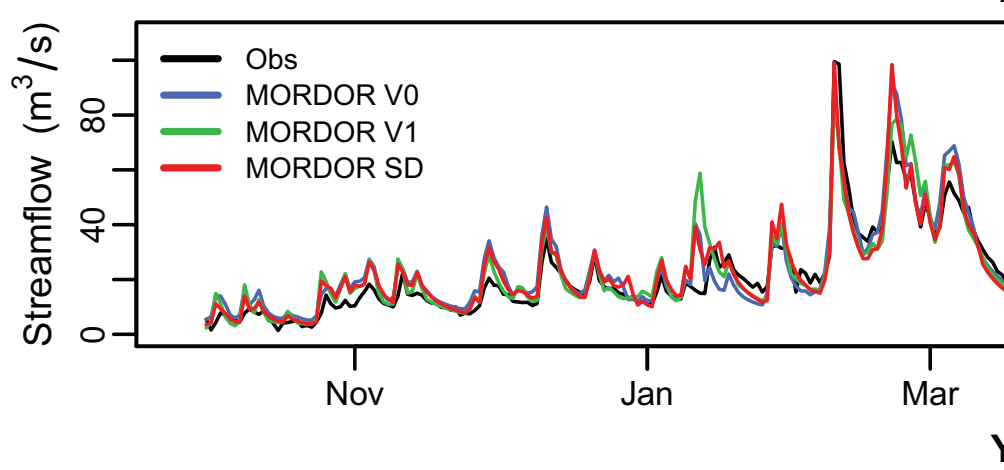

Year 1999

May

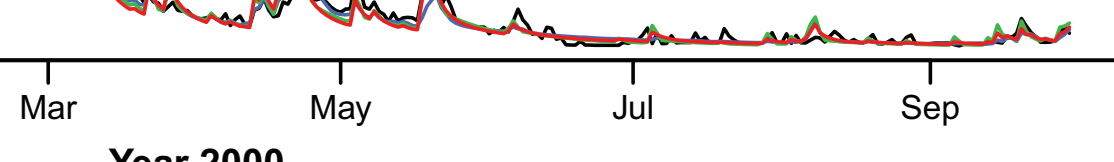

Year 2000
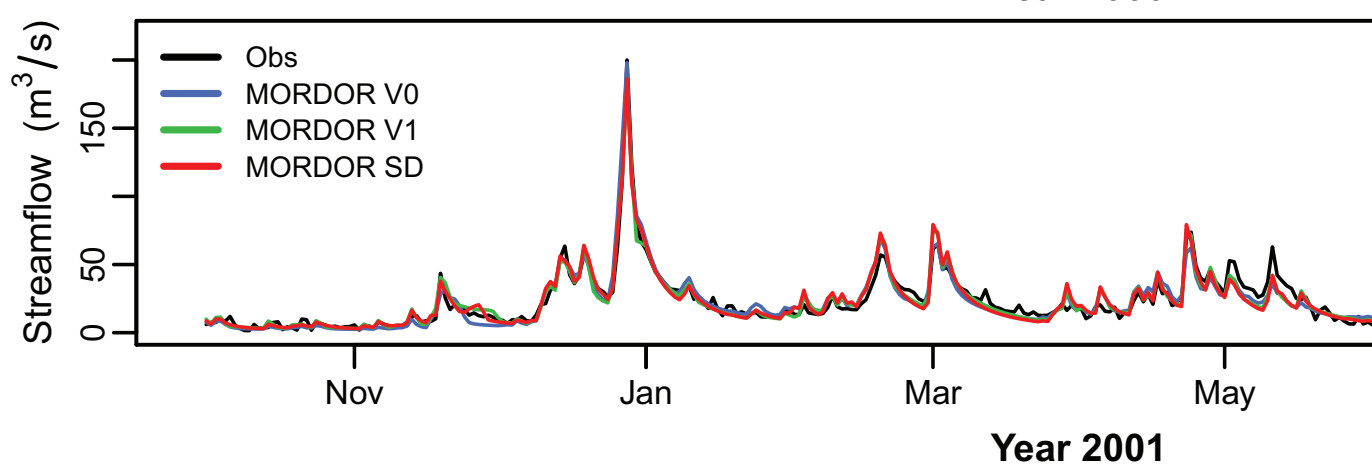

Year 2001
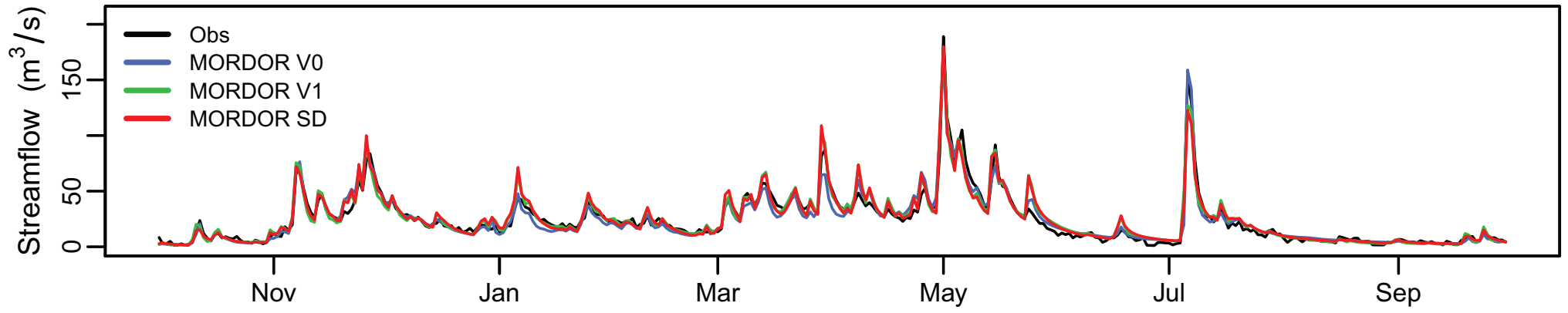

Year 2002

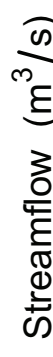

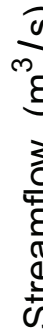

$-$
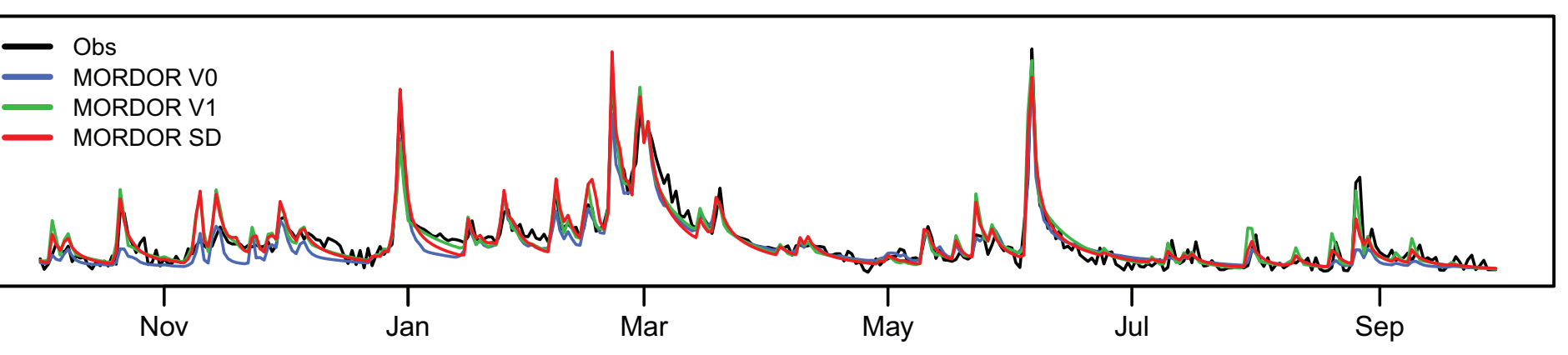


\section{Dordogne@Bort}

\section{Year 1986}
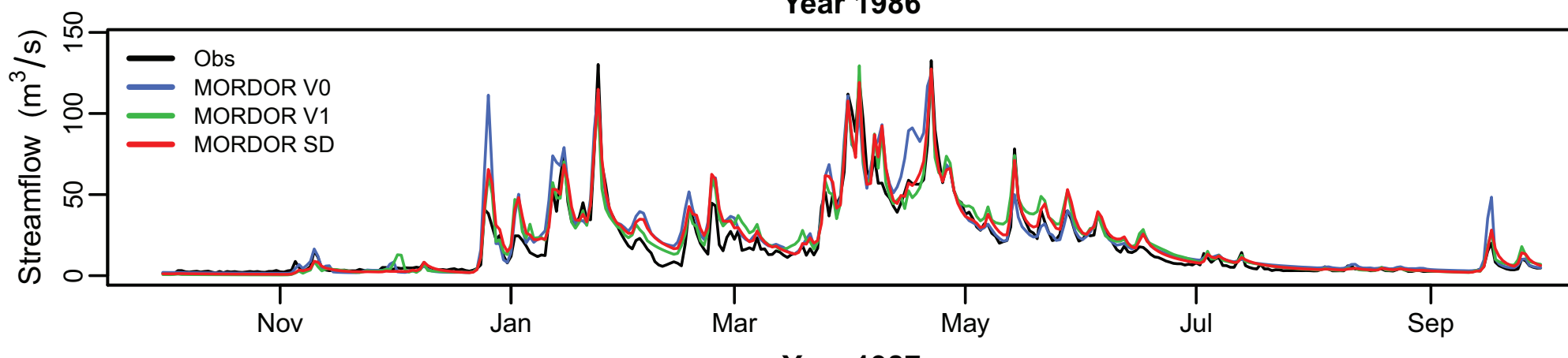

Year 1987

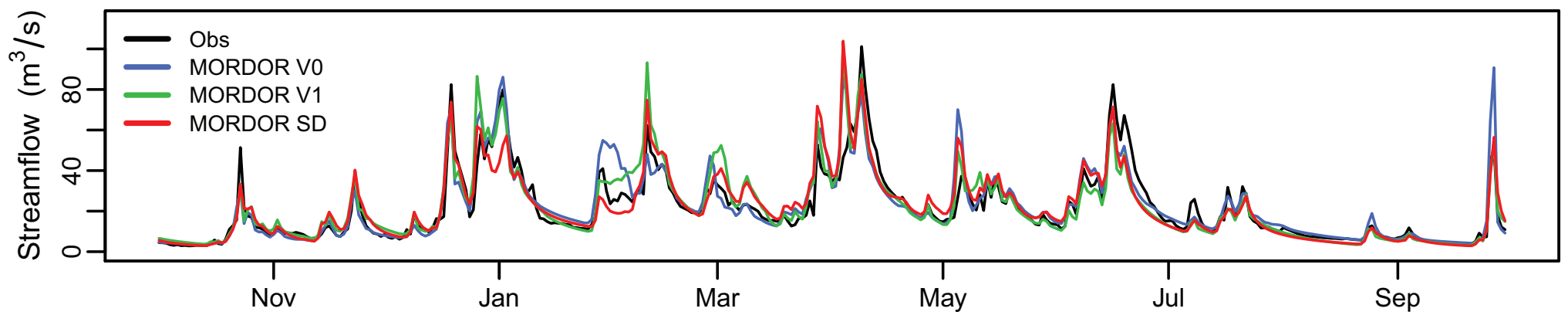

Year 1988

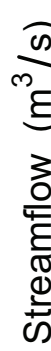

$-\sqrt{-} \begin{aligned} & \text { Obs } \\ & \text { MORDOR V0 } \\ & \text { MORDOR V1 }\end{aligned}$

- - MORDOR VO

$3 \stackrel{\circ}{\leftarrow} \longrightarrow$ MORDOR SD

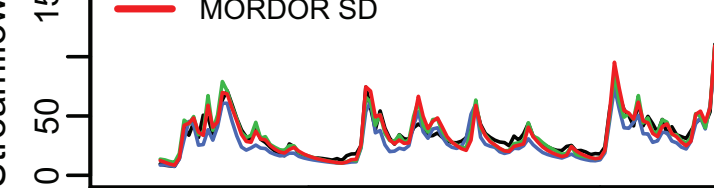
Nov
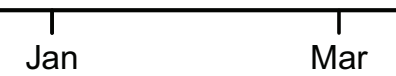

Year 1989

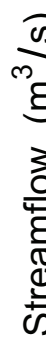

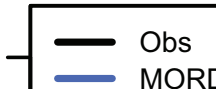

MORDOR V1

- MORDOR SD

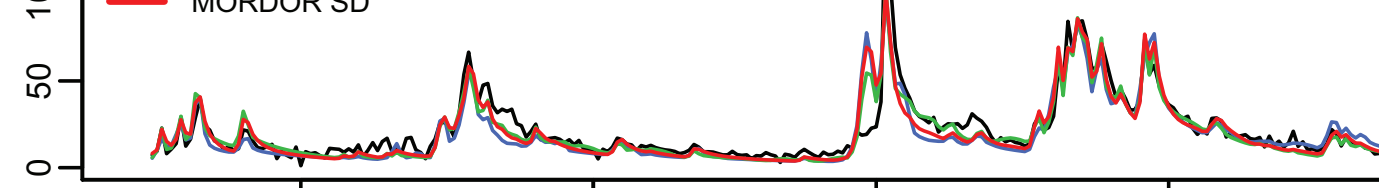
Nov

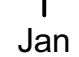

Mar

Year 1990

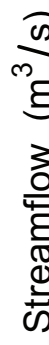

Obs

- MORDOR V0

ก- MORDOR V1

MORDOR SD

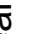

은

cumber

Nov
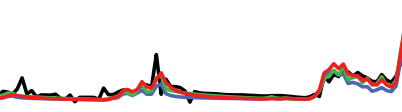

An a

Mar

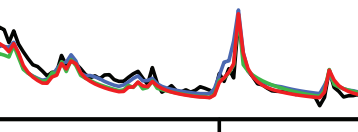

Jul

Sep

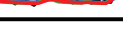

Jan

Year 1991
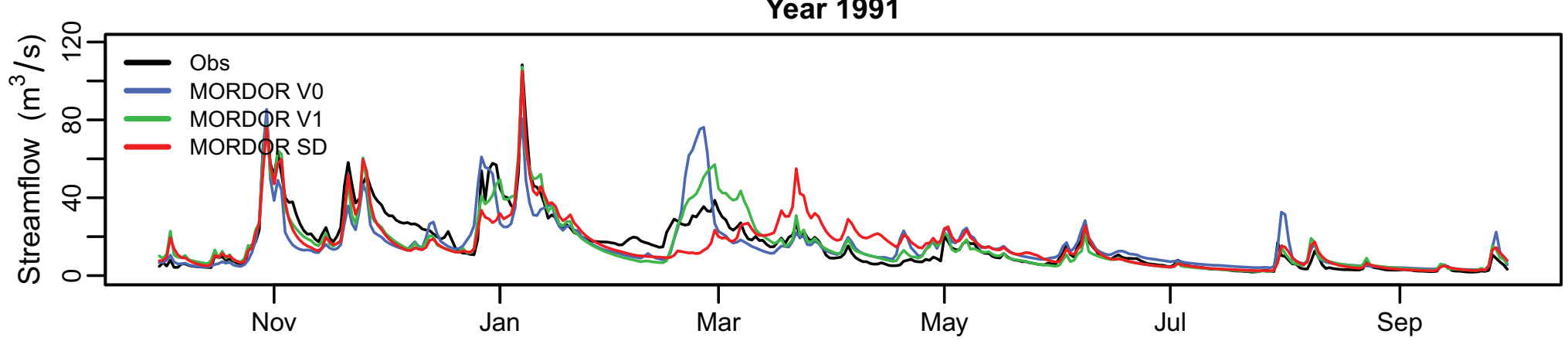
Dordogne@Bort

Year 1992
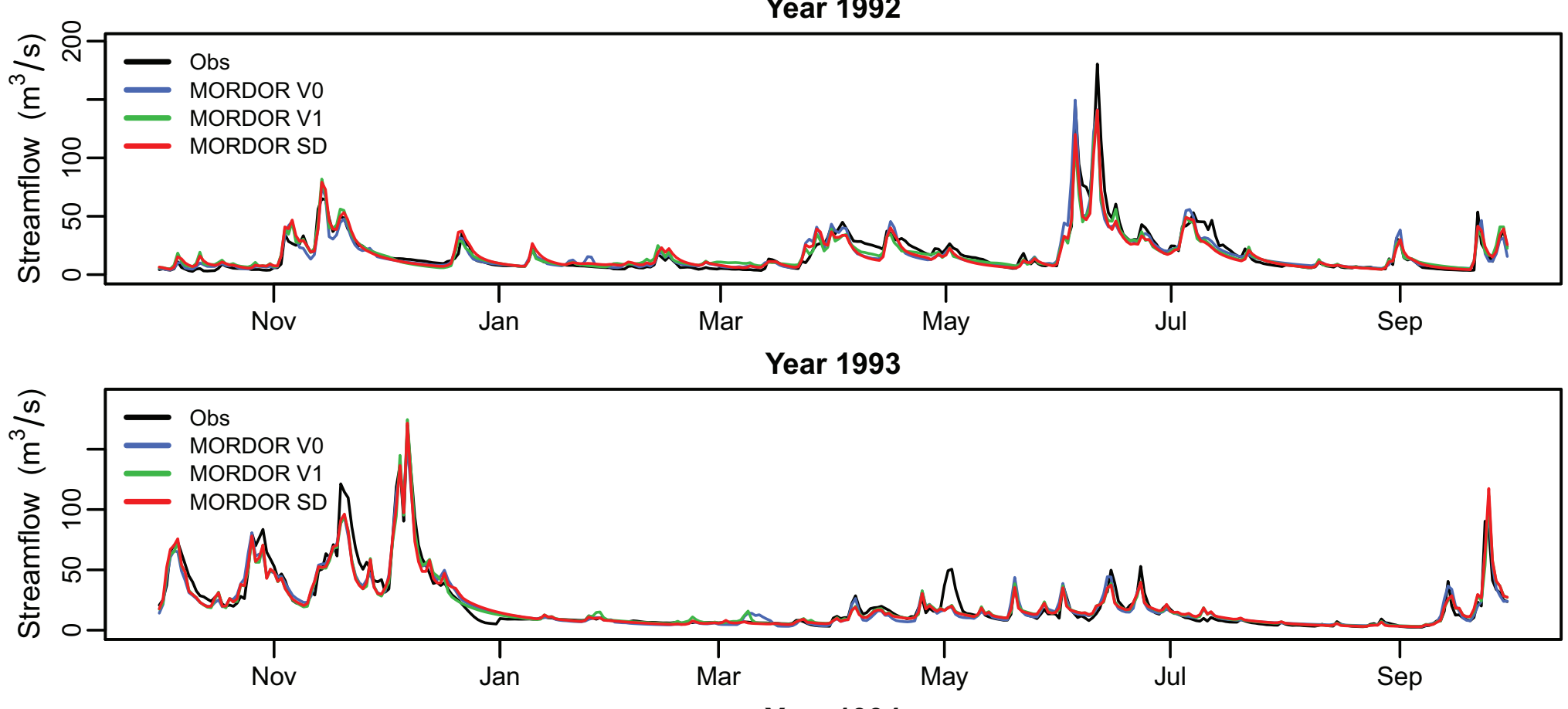

Year 1993
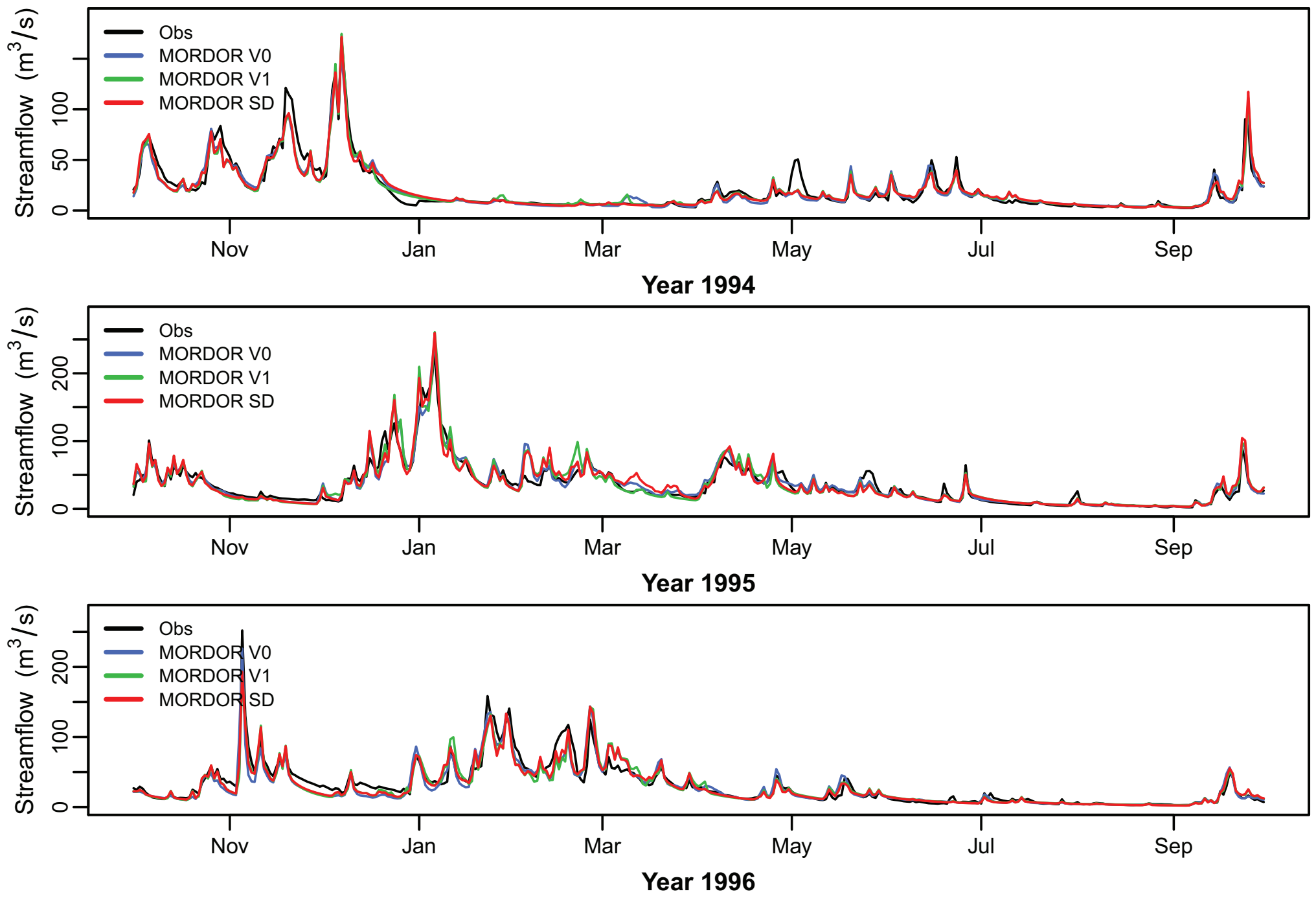

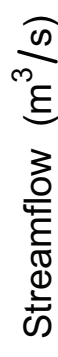

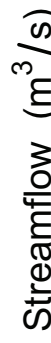




\section{Dordogne@Bort}

\section{Year 1998}
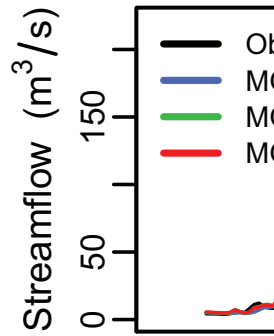

Obs

MORDOR V0

MORDOR V1

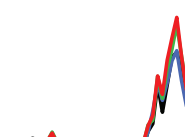

Mamind

Nov
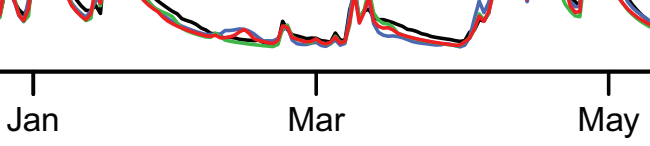

Year 1999

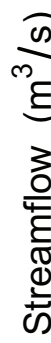

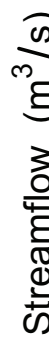

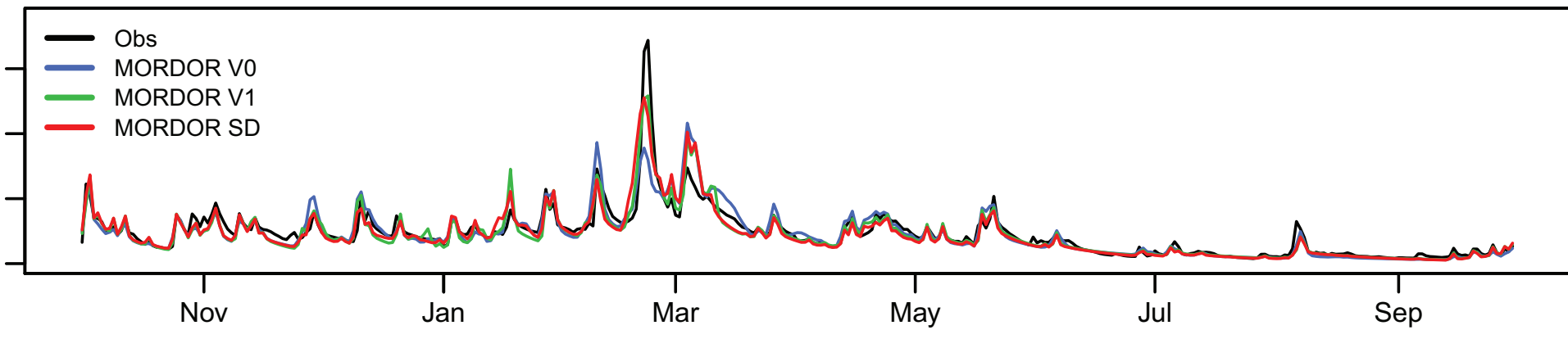

Year 2000
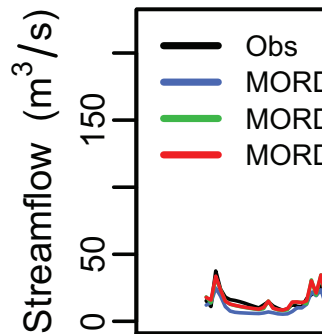

- MORDOR VO

— MORDOR SD

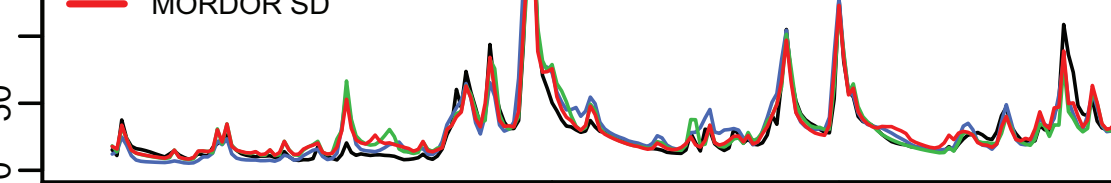

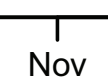

Jan

Mar

Year 2001

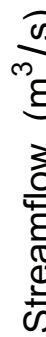

- Obs

- MORDOR VO

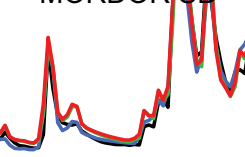
Wha

Nov

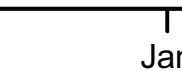

dann

Mathed

Jan Mar

Year 2002

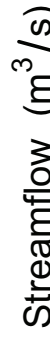

$\longrightarrow$ Obs

MORDOR VO

MORDOR V1

$8-1$ MORDOR SD

象

ㄱ-

M

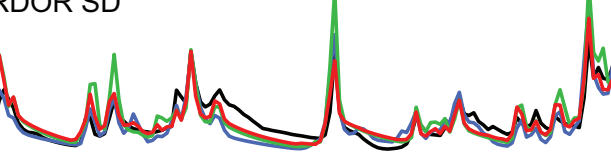

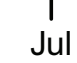

Sep

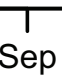




\section{Doubs@Brenet}

Year 1999

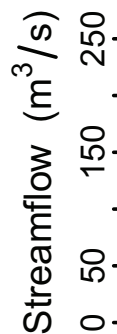

三
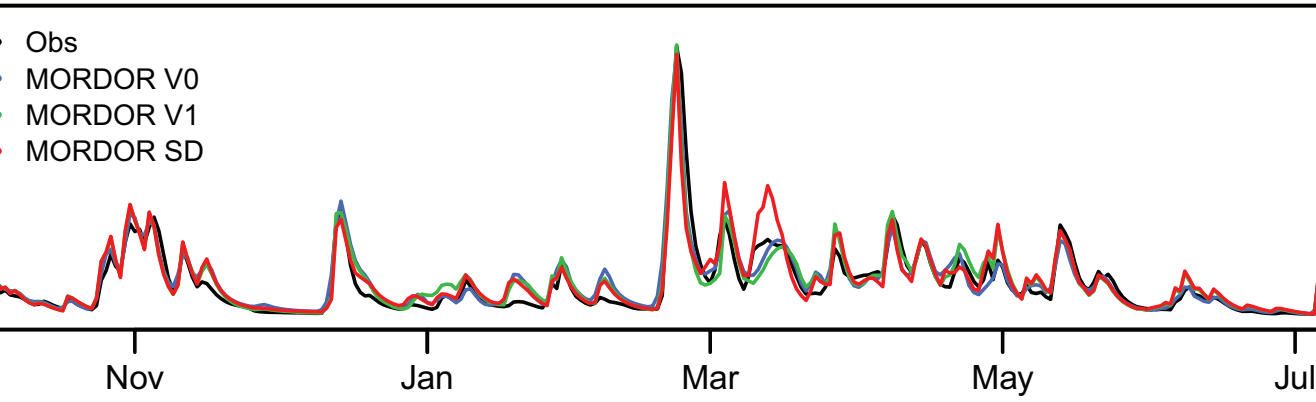

Year 2000
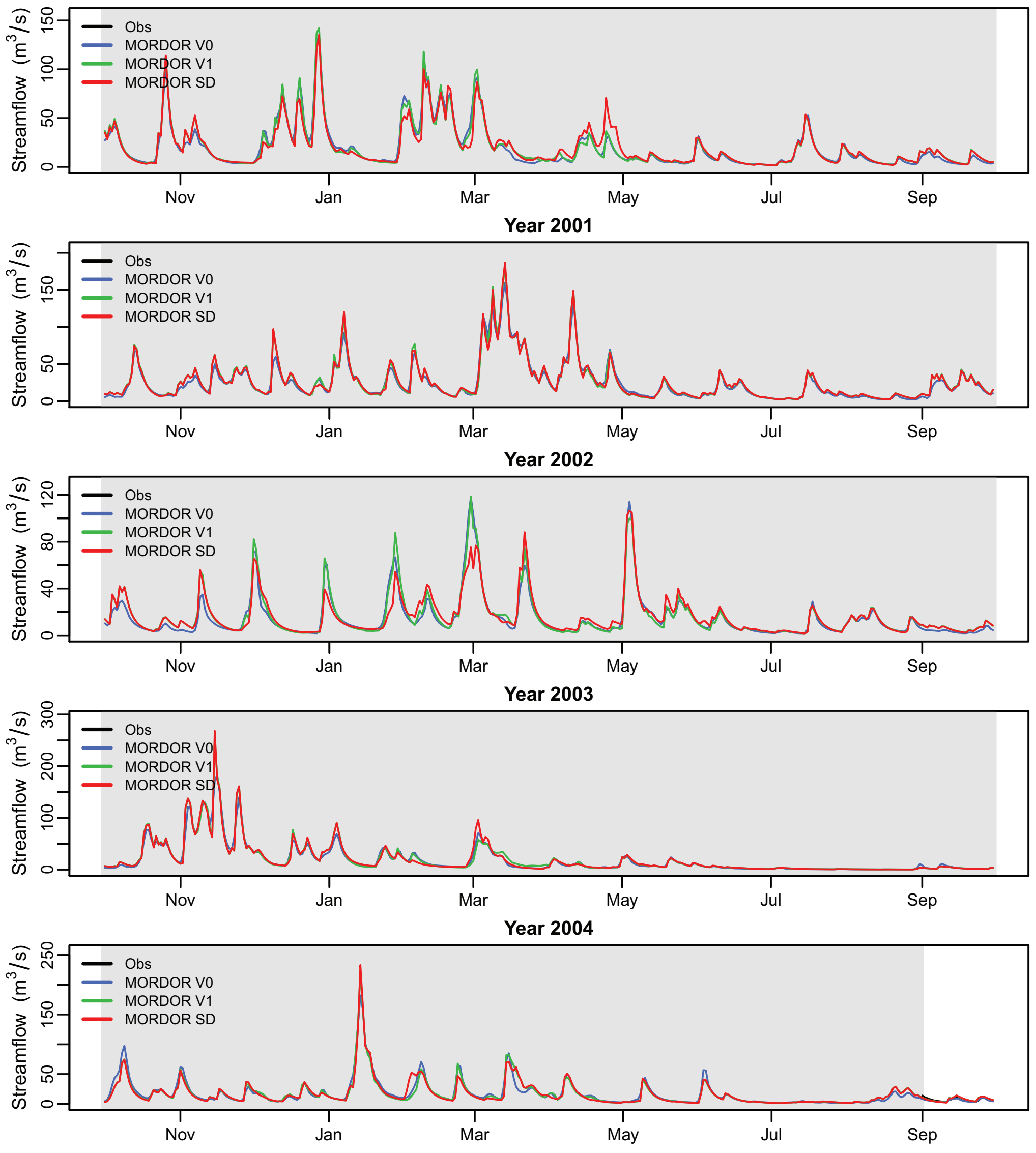


\section{Doubs@Neublans}

Year 1992
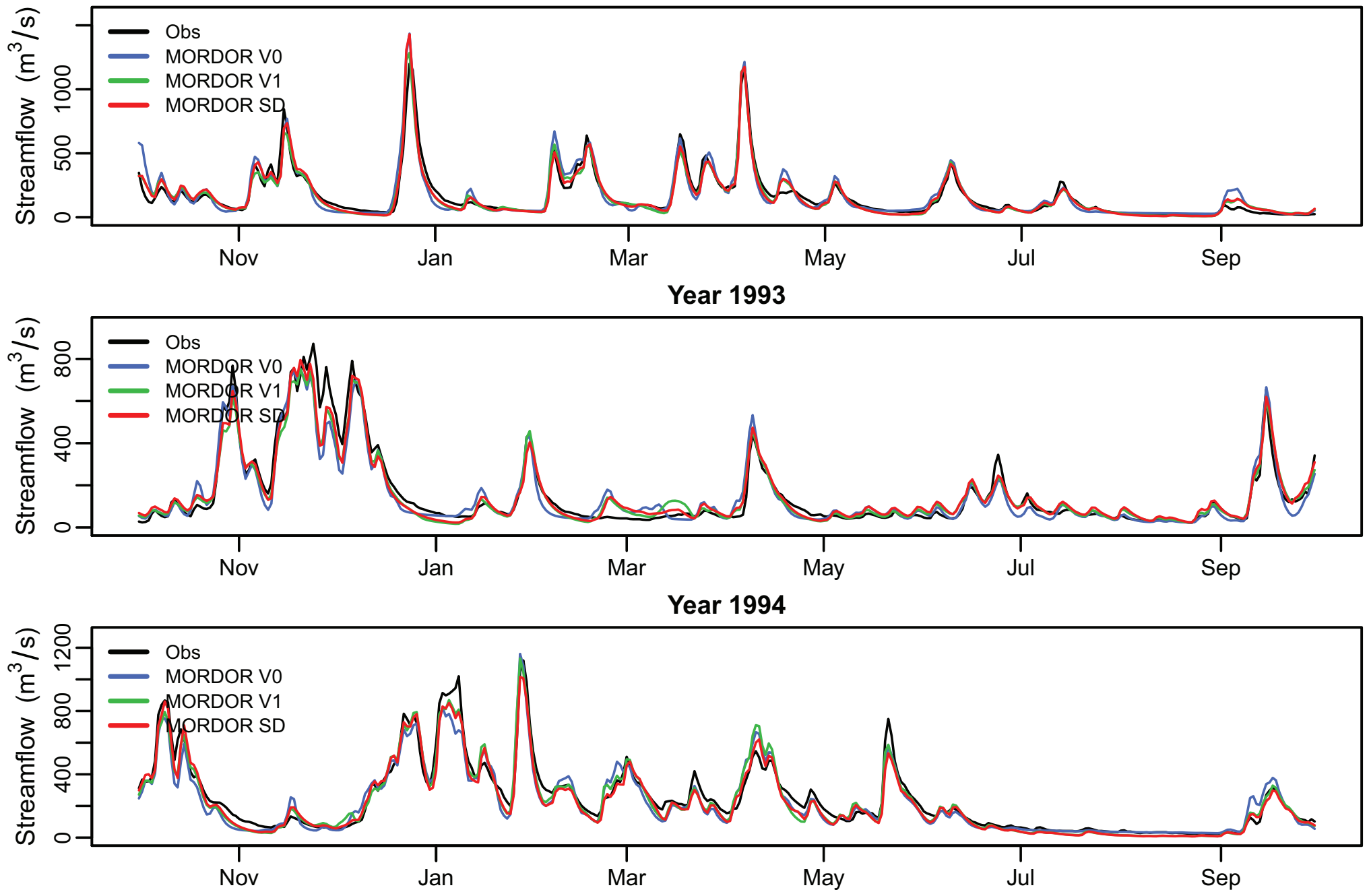

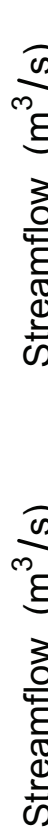

Year 1995
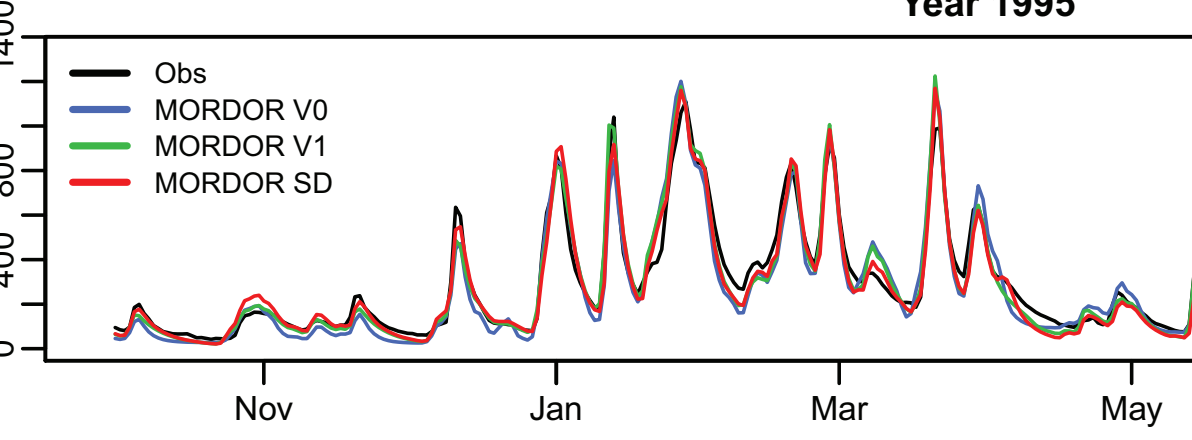

Year 1996
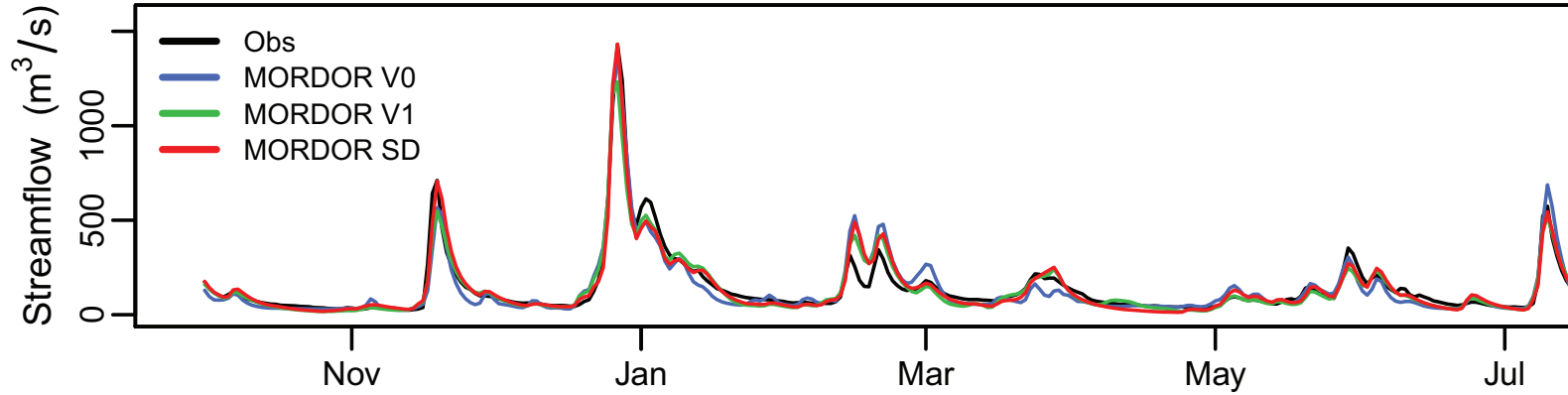

Year 1997
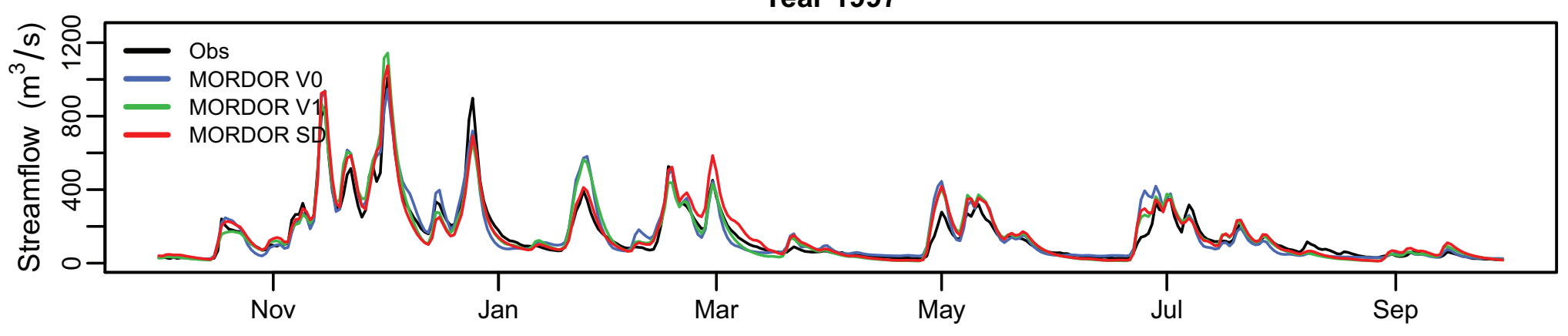


\section{Drac@Sautet}

\section{Year 1976}

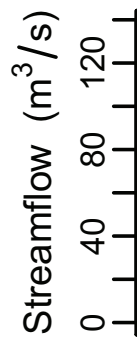

- Obs

MORDOR V0

$\longrightarrow$ MORDOR V1

(axio

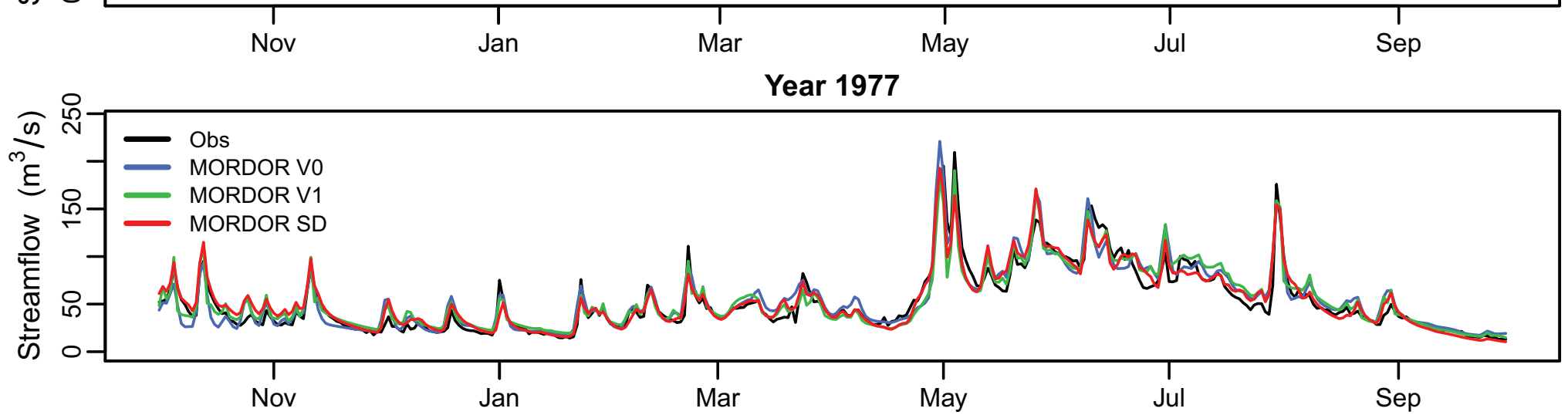

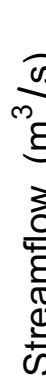

Year 1978

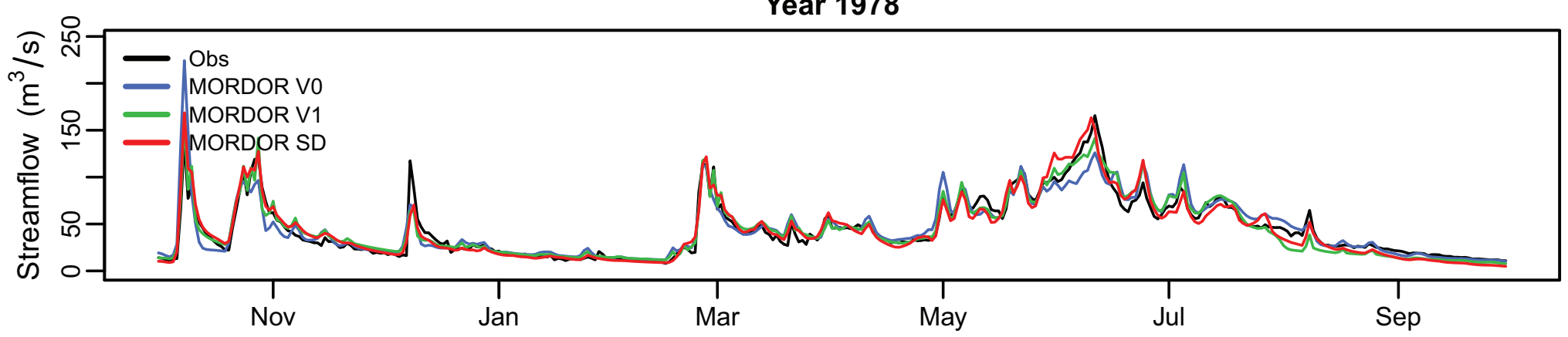

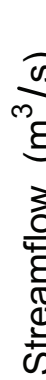

है

Year 1979
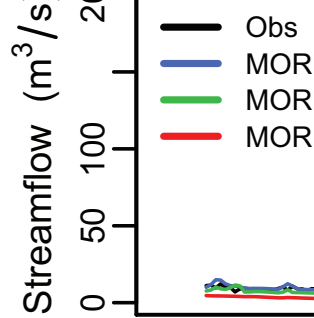

MORDOR VO

- MORDOR SD

酷 .
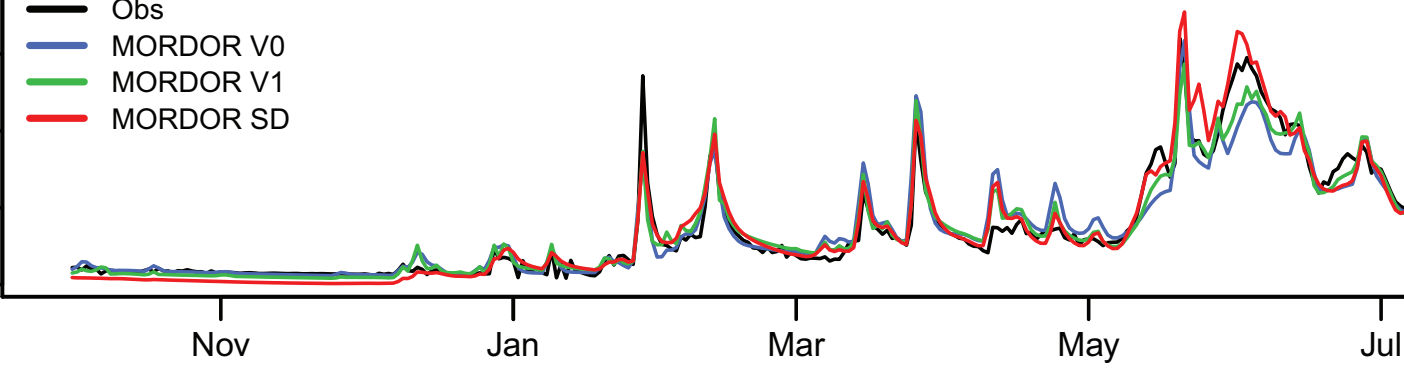

Year 1980
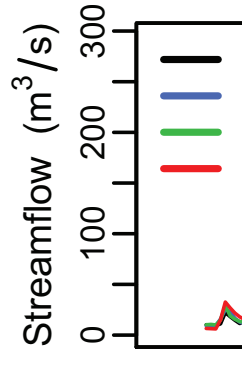

Obs

MORDOR V0

MORDOR V1

MORDOR SD

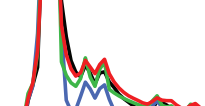

M

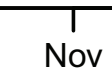

$\therefore$ and

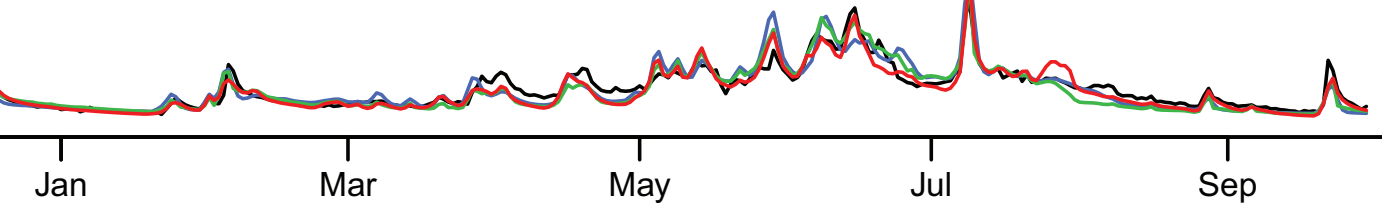

Year 1981
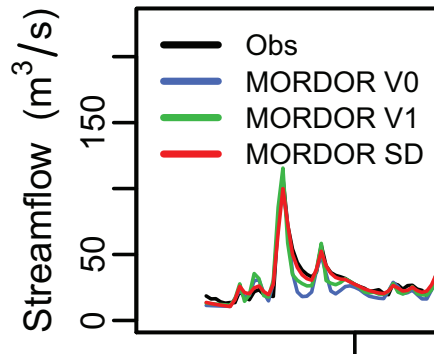


\section{Durance@Clapiere}

Year 1989
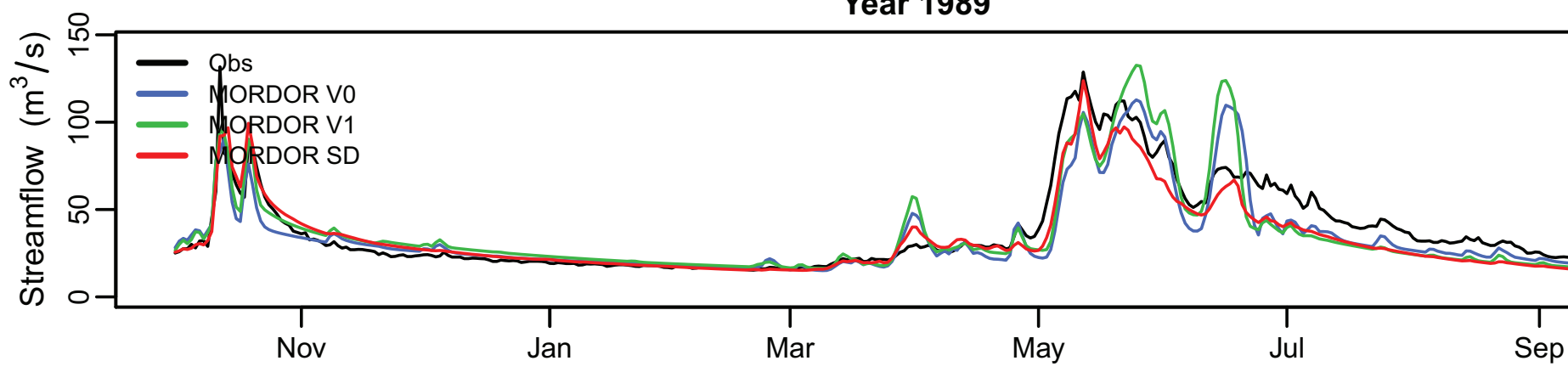

Year 1990
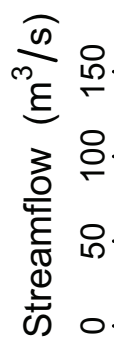

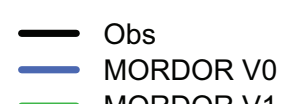

- MORDOR V1

- MORDOR SD
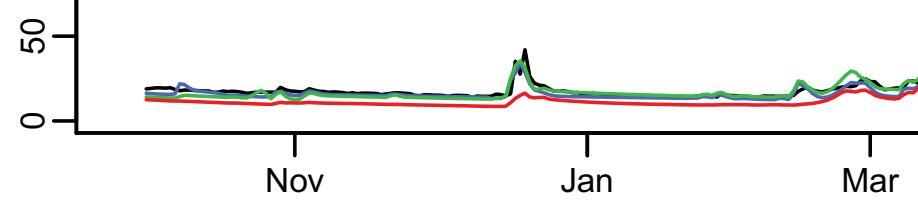

Year 1991

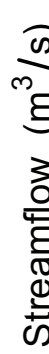

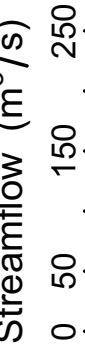

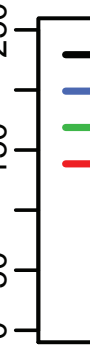

- Obs

- MORDOR V0

MORDOR V1

- MORDOR SD

ind

Nov

(n)

a
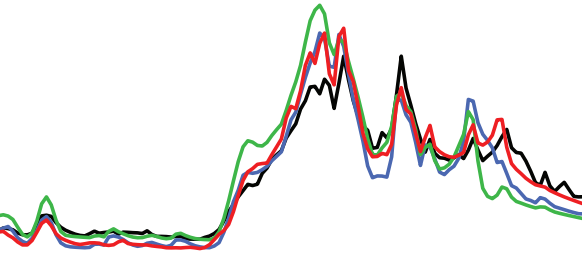

Jul
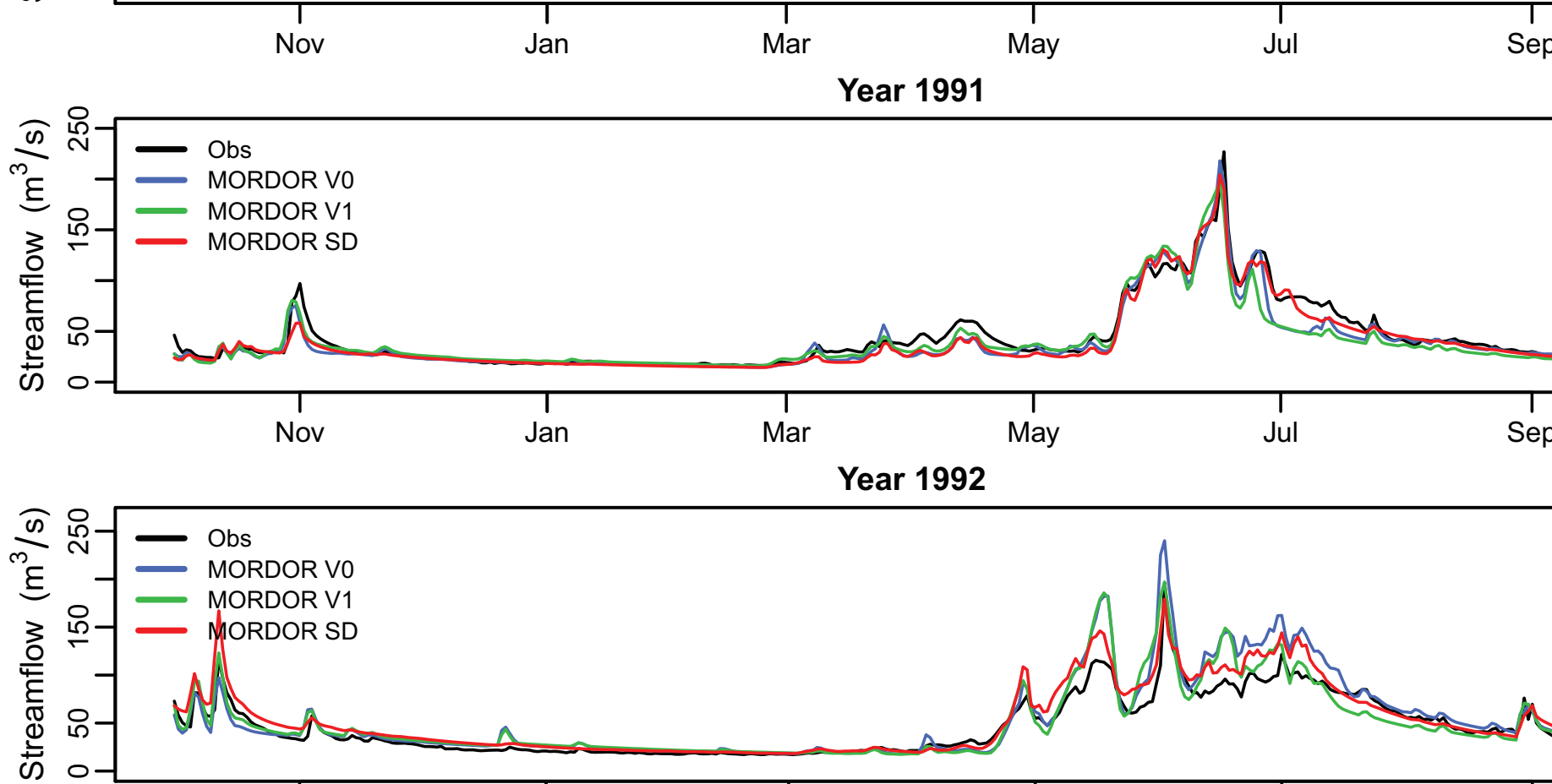

Year 1992

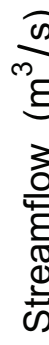

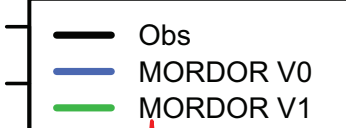

- MORDOR SD

was .
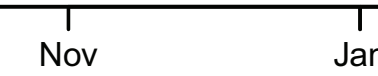

Jan

Mar

Year 1993

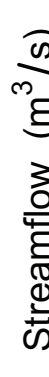

a

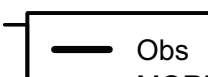

- MORDOR V0

MORDOR V1

(2)

오

Mara

a
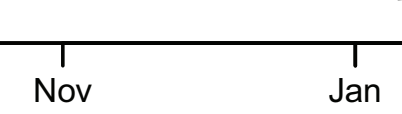

Jan

Mar

Year 1994
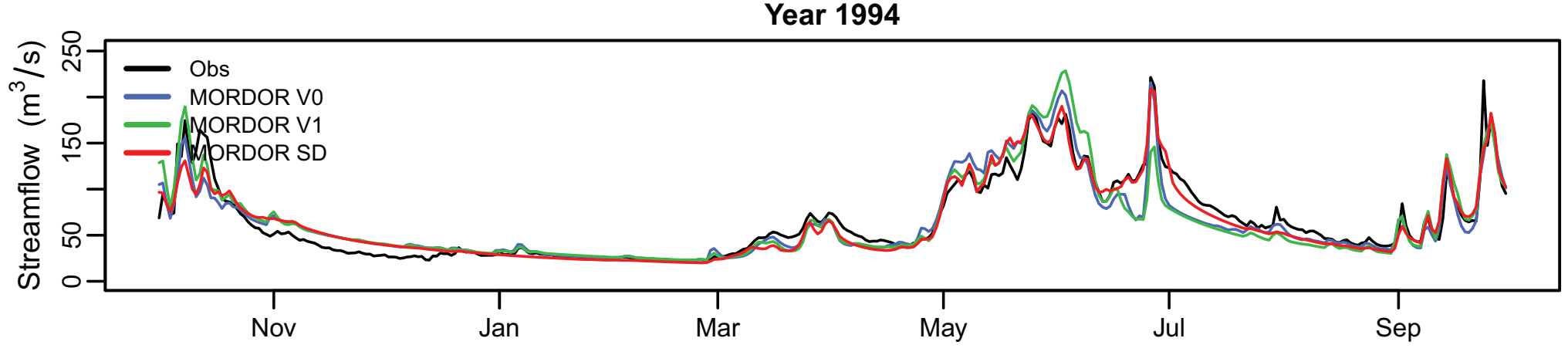


\section{Gage@Gagell}

Year 1993

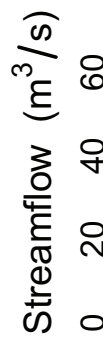

$\begin{array}{ll}- & \text { Obs } \\ & \text { MORDOR VO } \\ \text { MORDOR V } 1\end{array}$

- MORDOR SD

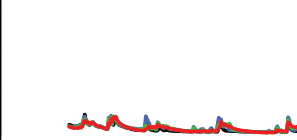
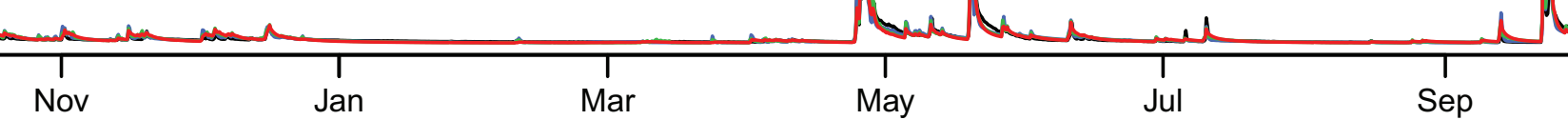

Jov Jan

Mar

Jul

Sep

Year 1994

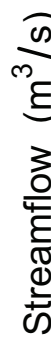

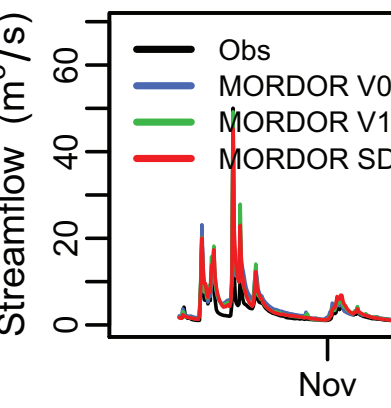

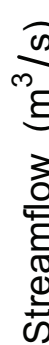
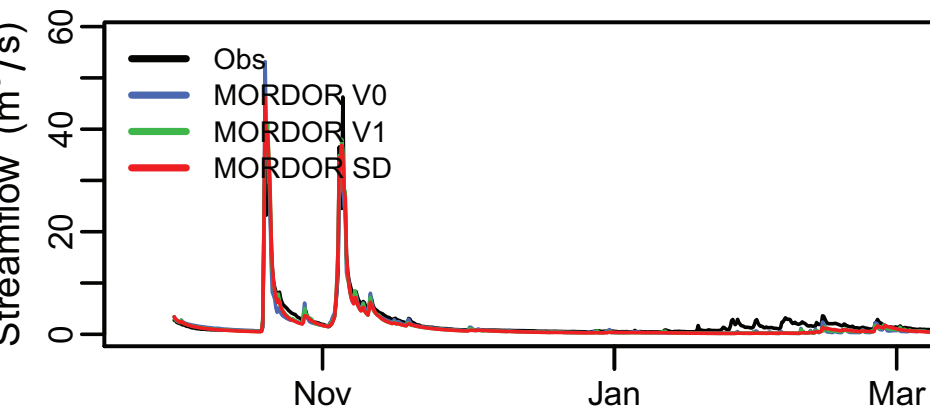

Year 1996
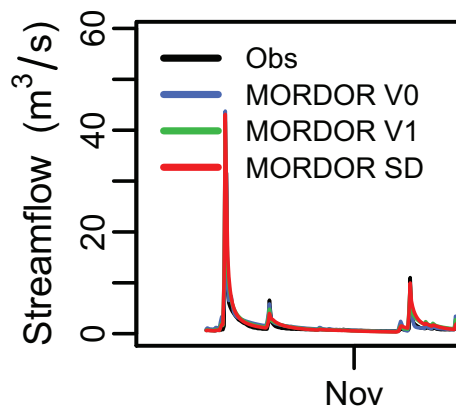

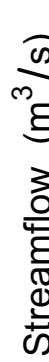

है

hu

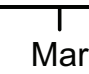

Year 1995
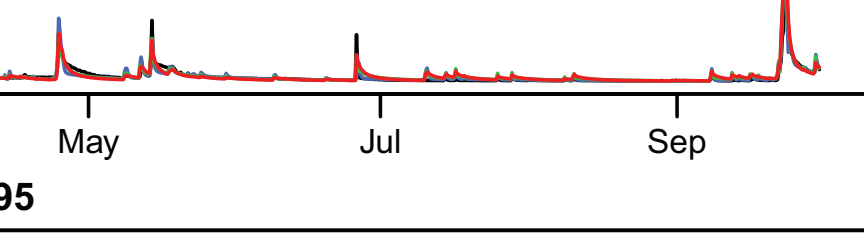


\section{Gage@Gagell}

Year 1999

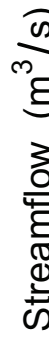

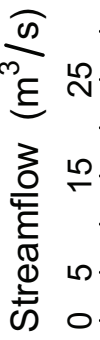

$\longrightarrow$ Obs

MORDOR VO

MORDOR V

MORDOR SD

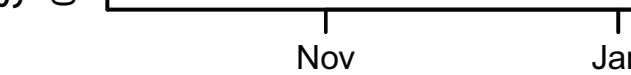

Jan Mar

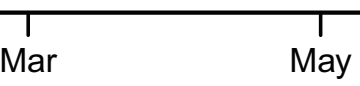

Jul

Year 2000
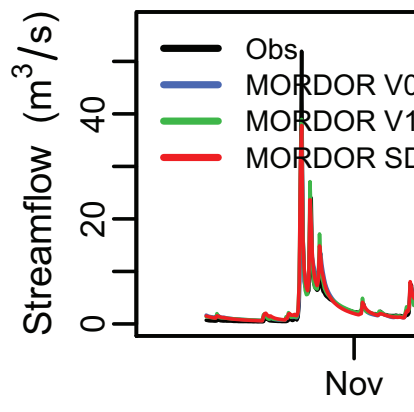
. 
Gardon@Corbes

Year 1993

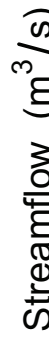

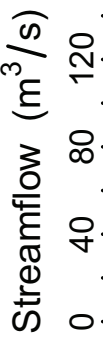

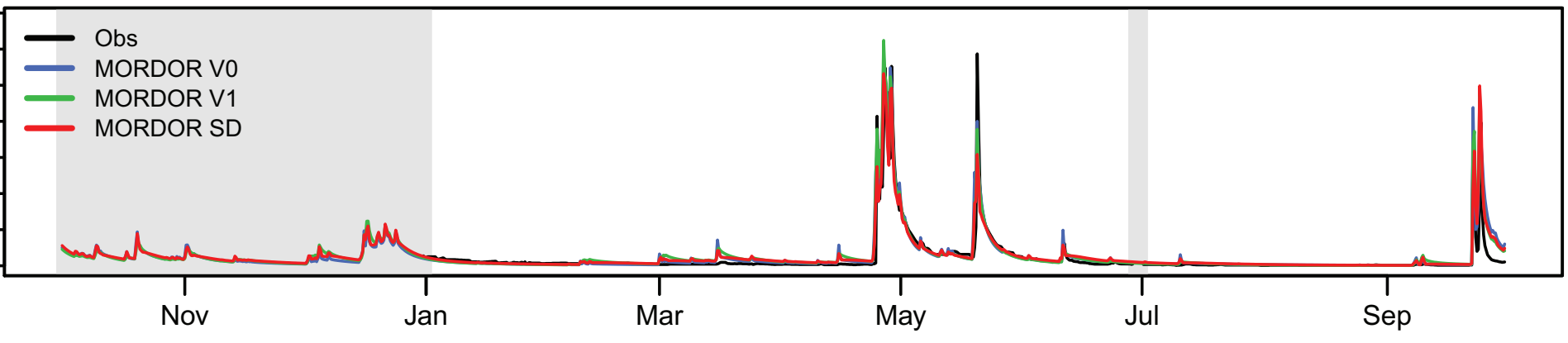

Year 1994

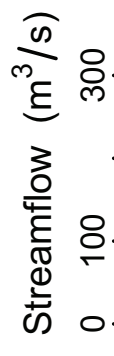

- Obs

MORDOR V0

8-

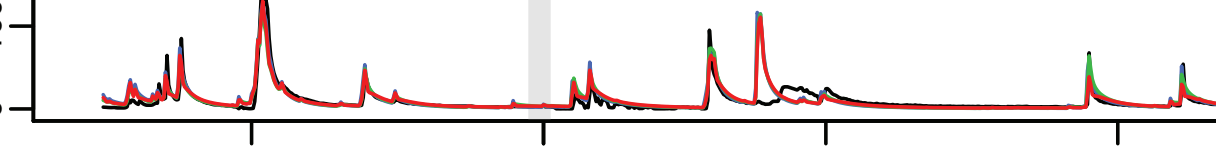

Nov

Jan

Mar

Year 1995

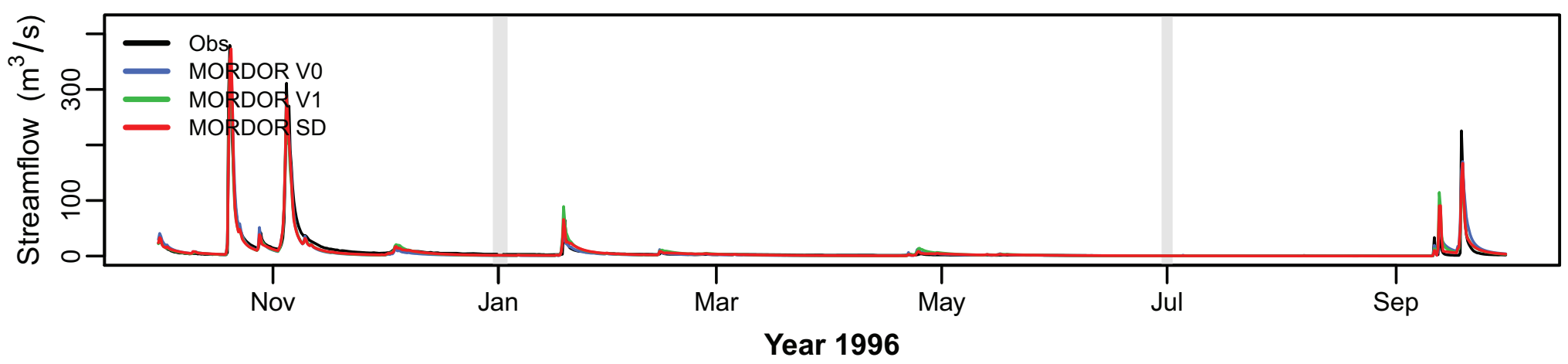

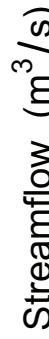

Obs

MORDOR V0

MORDOR SD

MORDOR SD

Year 1997

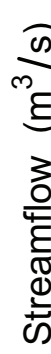

- Obs

西

요

MORDOR V

MORDOR SD

o-ting

1 uㅐ WN N Nov

Jan

Mar

Year 1998
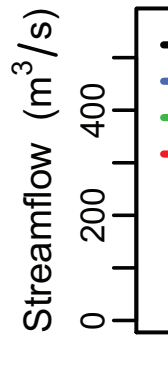

- Obs

- MORDOR V0

- MORDOR V1

- MORDORSD

11 
GaveEstaube@Gloriettes

Year 1988
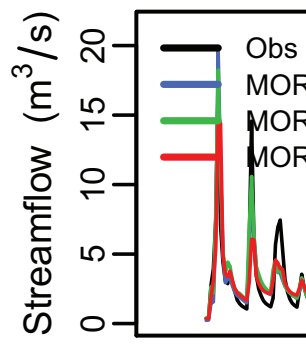

bs

ORDOR V1

MORDOR SD

mo
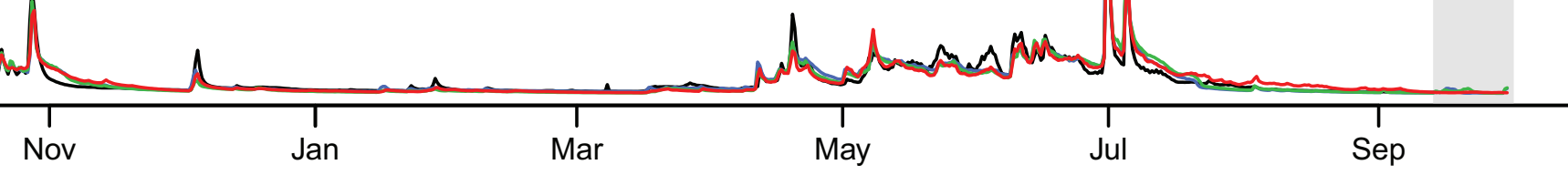

Year 1989

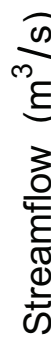

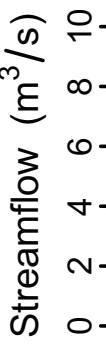

- Obs

- MORDOR VO

- MORDOR V1

- MORDOR SD

$-1$

Nov

Nov

Jan

Mar

Year 1990

$\frac{\pi}{0}$
m
5
3
0
0
$\frac{\pi}{4}$
0
0
0

- Obs

$\infty-$ MORDOR VO

- MORDOR V1

$0-$ MORDOR SD

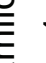

$-1$

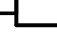

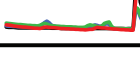

Nov
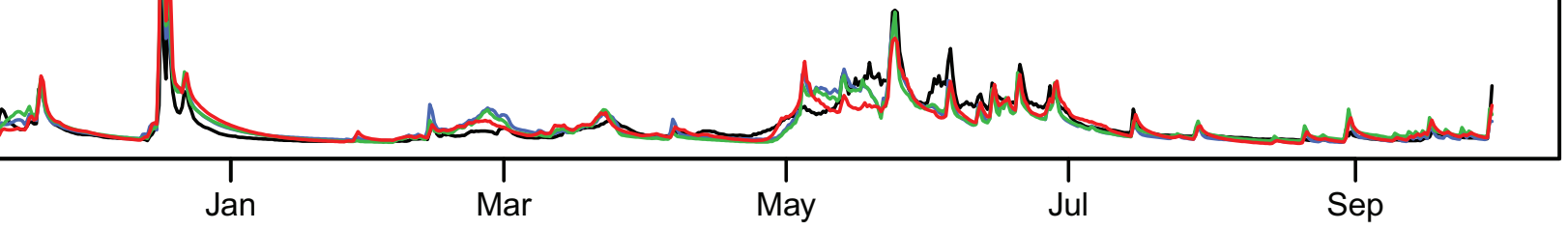

Jan

Year 1991
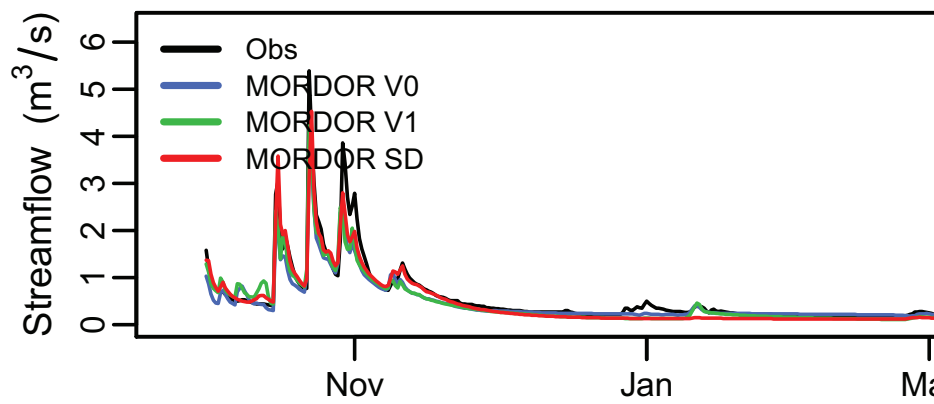

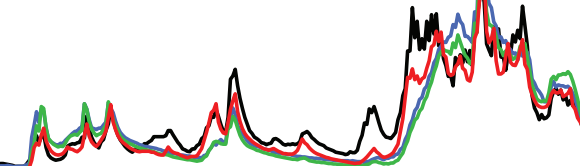
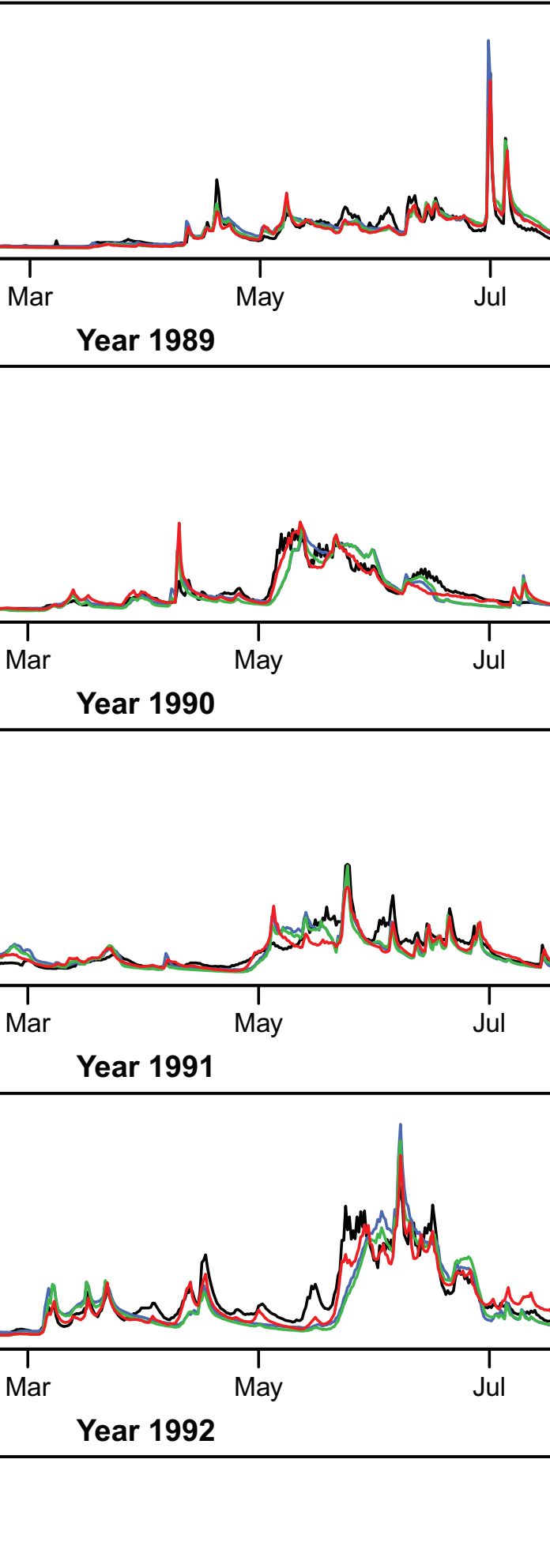

Year 1992

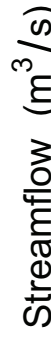

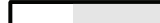
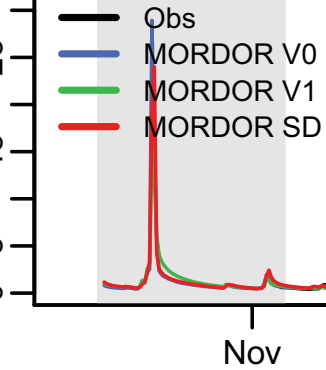

an

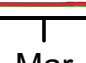

Mar

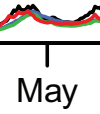

a

粮

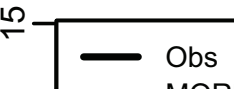

MORDOR V0

MORDOR V1

$\longrightarrow$ MORDOR SD

几

- Mnctaras 
GaveEstaube@Gloriettes

Year 1994

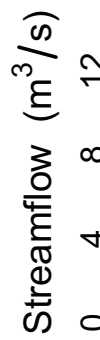

E

bs
ORDOR VO
ORDOR V 1

ORDOR SD

Nov

Jan

Mar

,

Year 1995

क

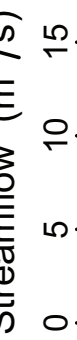

- Obs

- MORDOH vo

- MORDOH V1

MORDOR SD

$-\sin (h$

Nov

Jan Mar

Year 1996

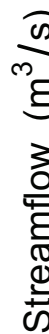

- Obs

ค- - MORDOR vp

으- MORDOR SP

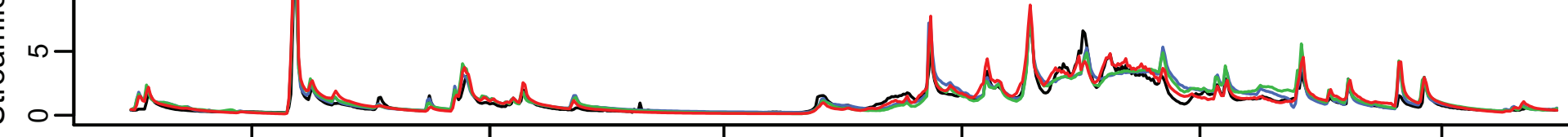

Nov

Jan

Mar

Year 1997

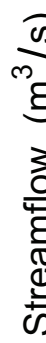

$\longrightarrow$ Obs

- MORDOR VO

- MORDOR V1

$\longrightarrow$ MORDOR SD

Medald d

Nov Jan

Jan Mar

Mar May

Year 1998

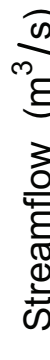

Obs

ㄱ- $\begin{aligned} & - \text { Obs } \\ & - \text { MORDORIV0 } \\ & \text { MORDOR V1 }\end{aligned}$

- MORDOR SD

-

(2)

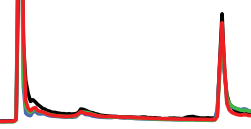

Nov

Jan

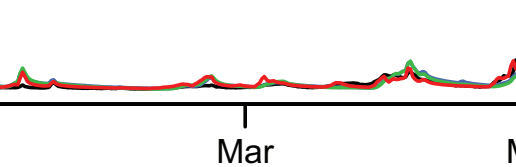

a gan

May

Sep

Year 1999

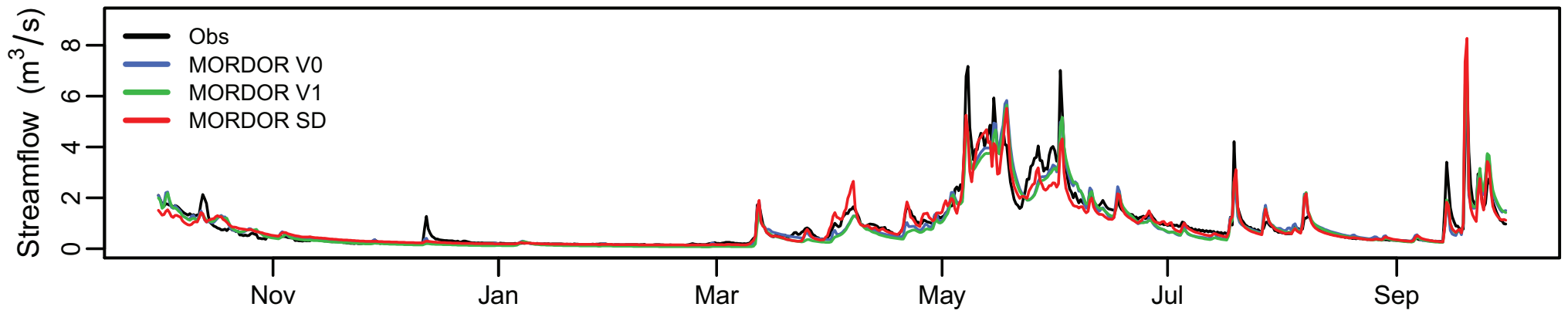


GaveEstaube@Gloriettes

Year 2006

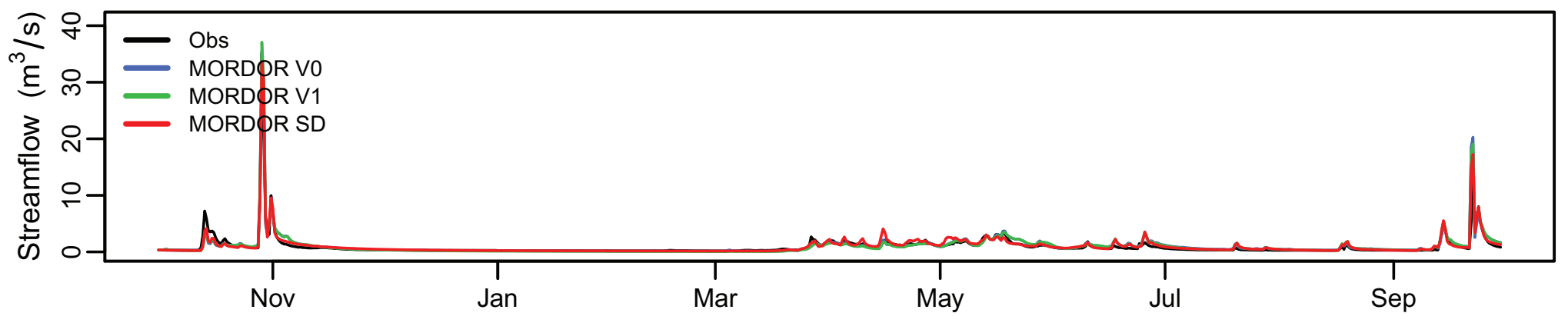




\section{Loire@Basset}

\section{Year 1975}
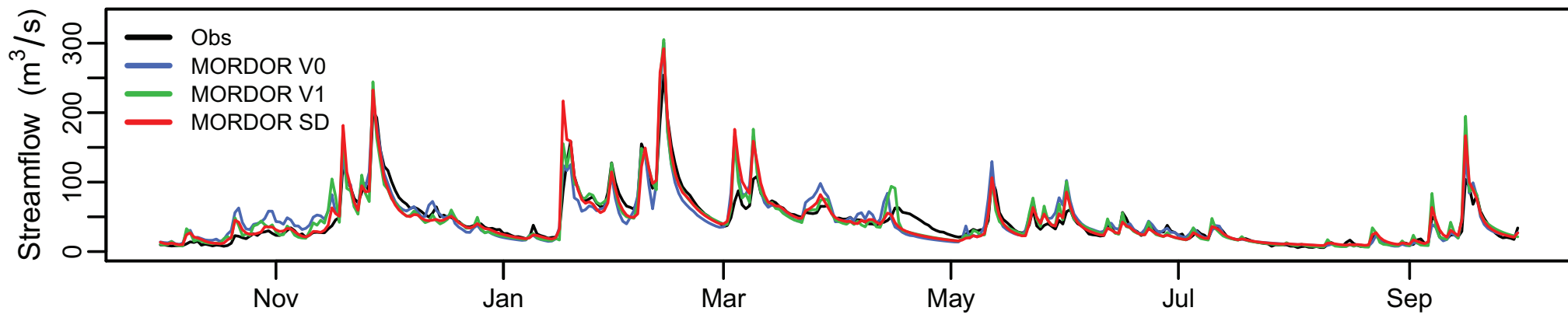

Year 1976
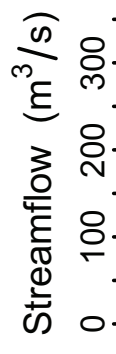

$\longrightarrow$ Obs

- MORDOR V0

MORDOR V1

- MORDOR SD

Nov

Jan Mar

Year 1977

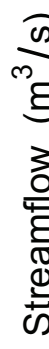

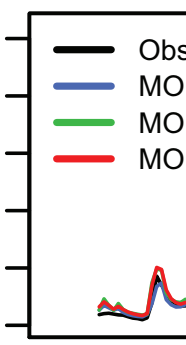

Obs

$R$ \ $y 0$

R $\$$

NW

Nov

Mar

Year 1978

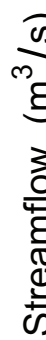
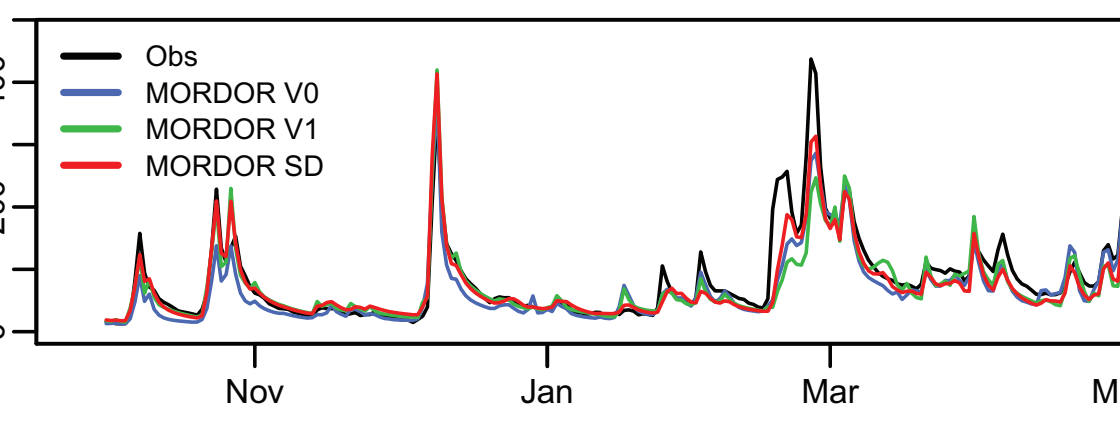

Year 1979
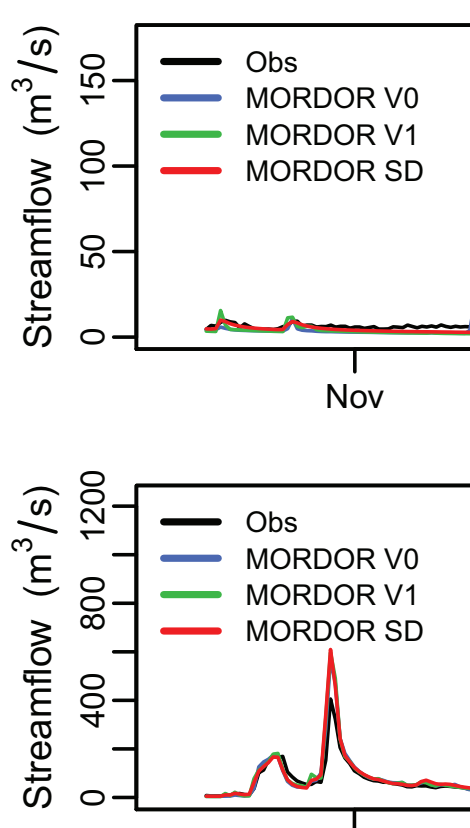

(

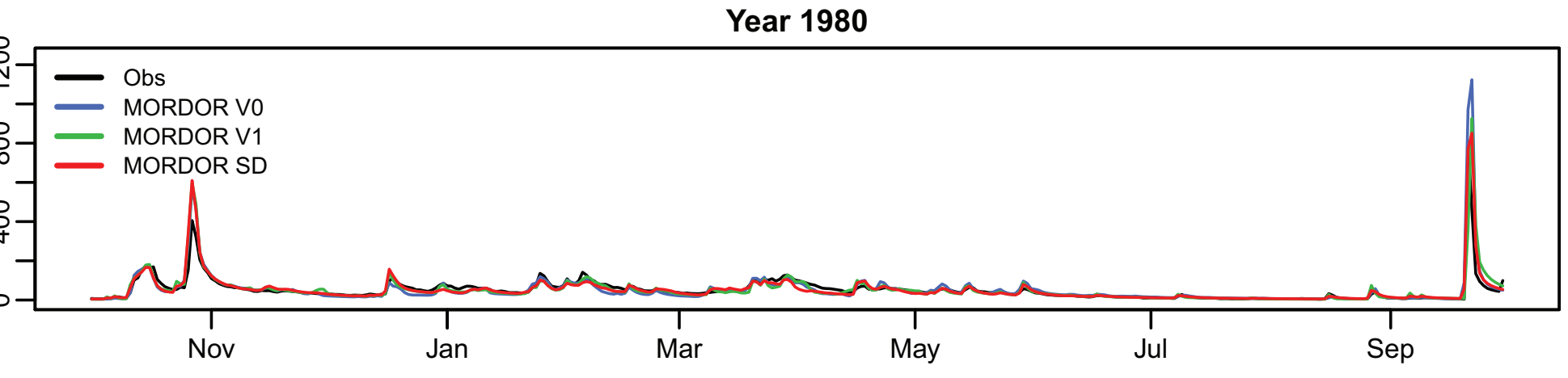




\section{Loire@Basset}

Year 1987

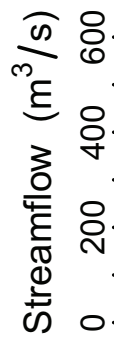

$\longrightarrow$ Obs

MORDOR VO

$\longrightarrow$ MORDOR V1

MORDOR SD

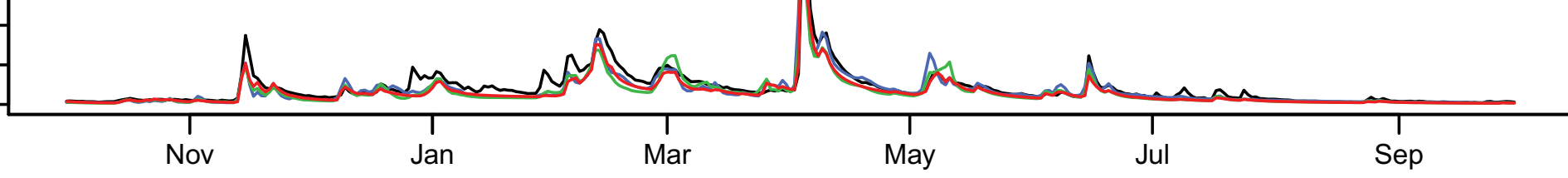

Year 1988
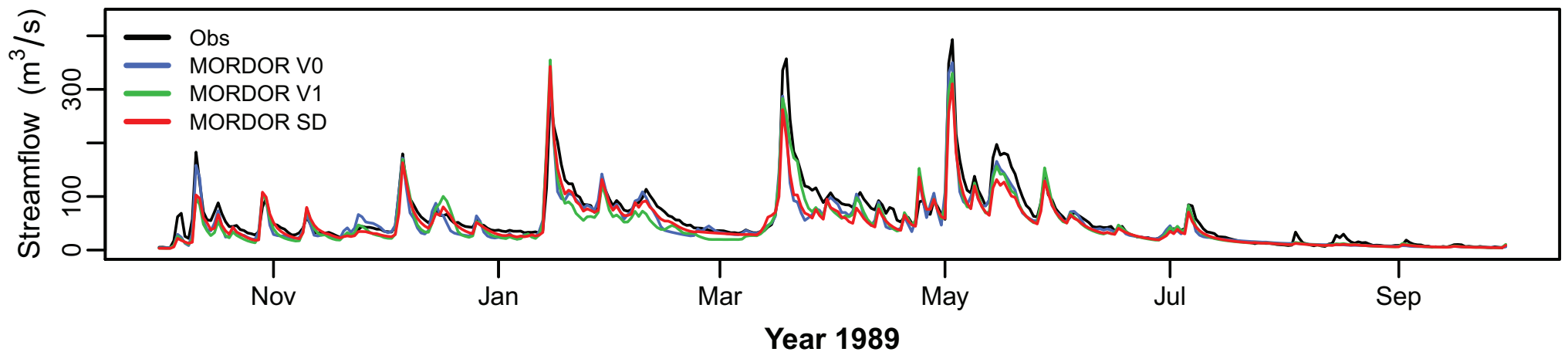

$\frac{\pi}{m}$
कह
3
0
$\frac{0}{0}$
0
0
0

Obs
- MORDOR V0
MORDOR V1

- MORDOR SD

8-

0

Nov

M

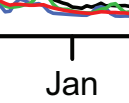

Year 1990

$\frac{0}{0}$
m
$\xi$
3
0
5
0
0
0
0

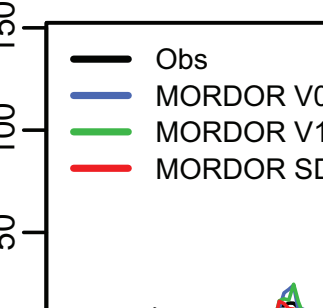

and N Nov

Jan
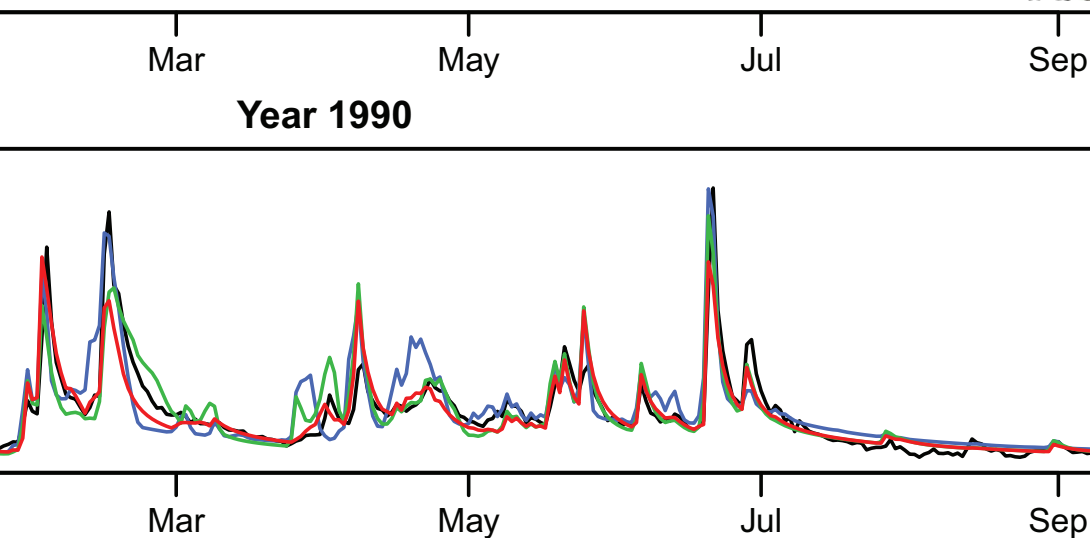

Sep

Year 1991
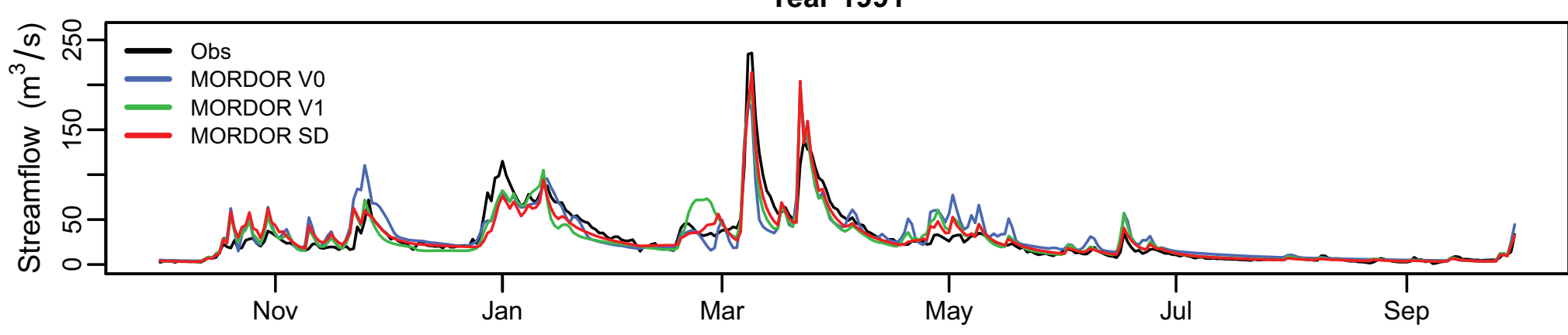

Year 1992

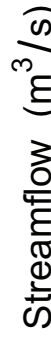

$=$

- MORDOR VO

- MORDOR V1

- MORDOR SD

Mama

人

and 


\section{Loire@Basset}

Year 1993

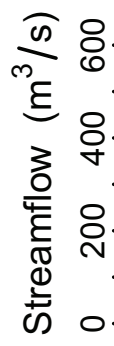

$\longrightarrow$ Obs

MORDOR V0

$\longrightarrow$ MORDOR V1

$\longrightarrow$ MORDOR SD

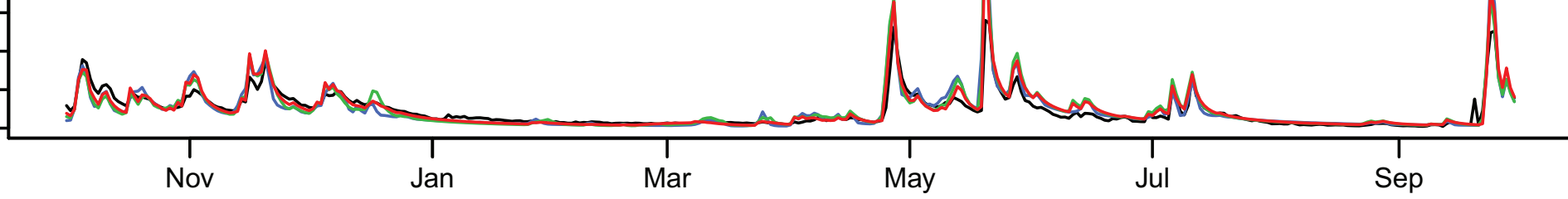

Year 1994
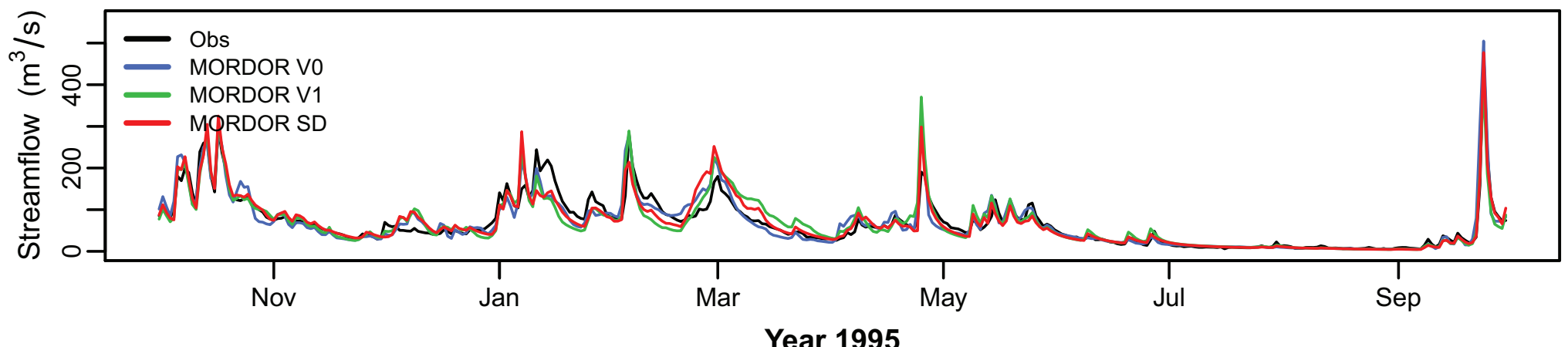

Year 1995
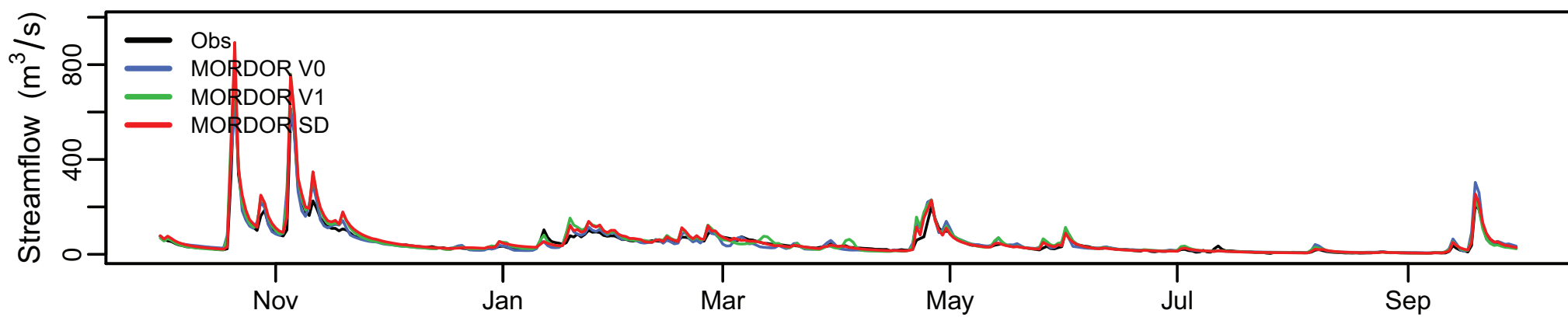

Year 1996
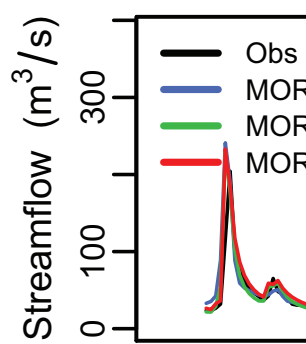

- MORDOR V0

- MORDOR V1

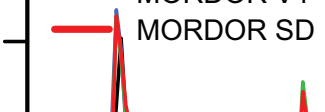
-

a

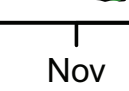

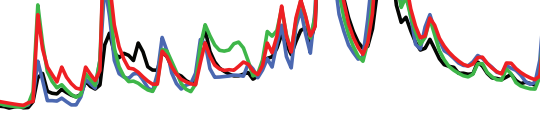

Nov

Jan

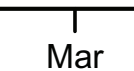

Mos Nan
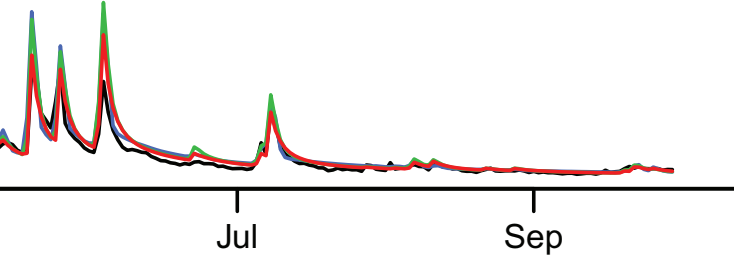

Year 1997
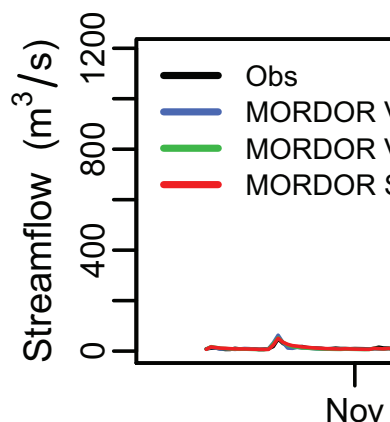

und
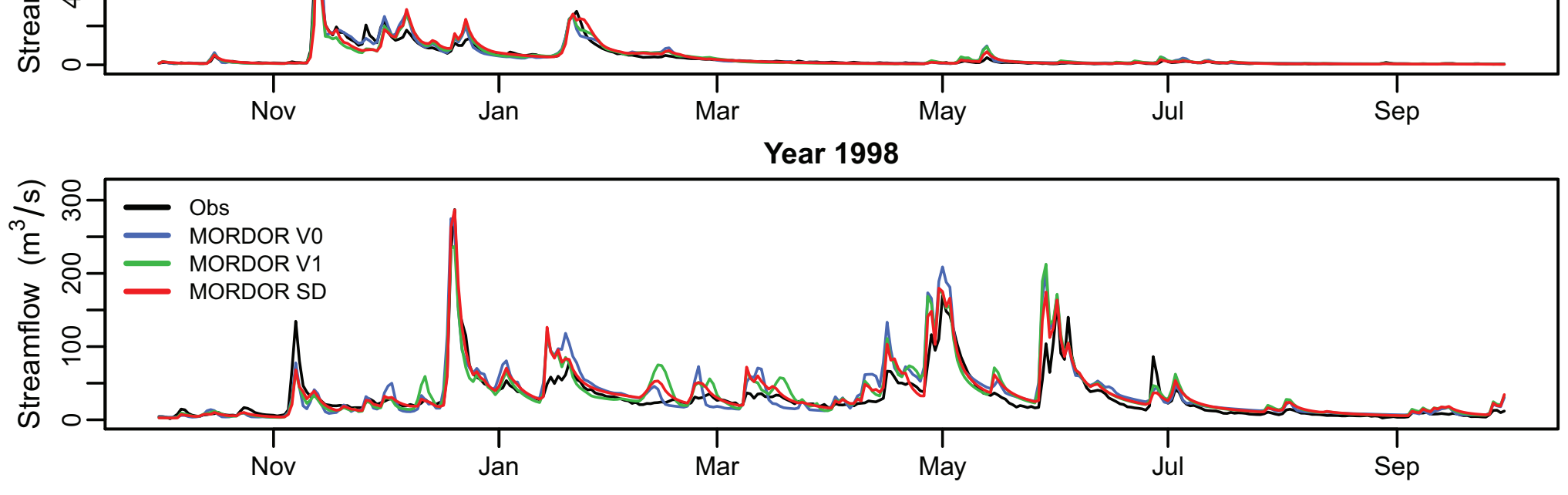


\section{Loire@LaPalisse}

Year 1999
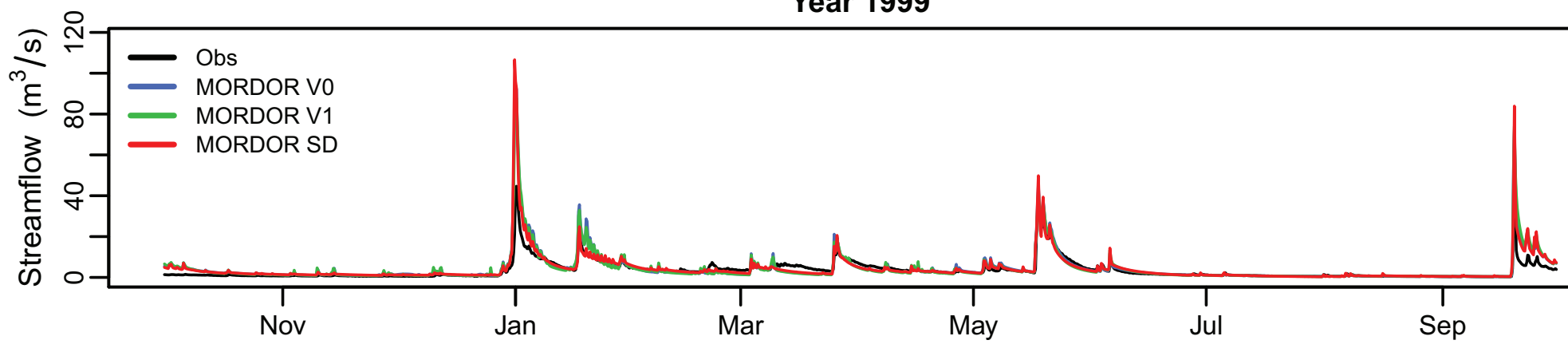

Year 2000
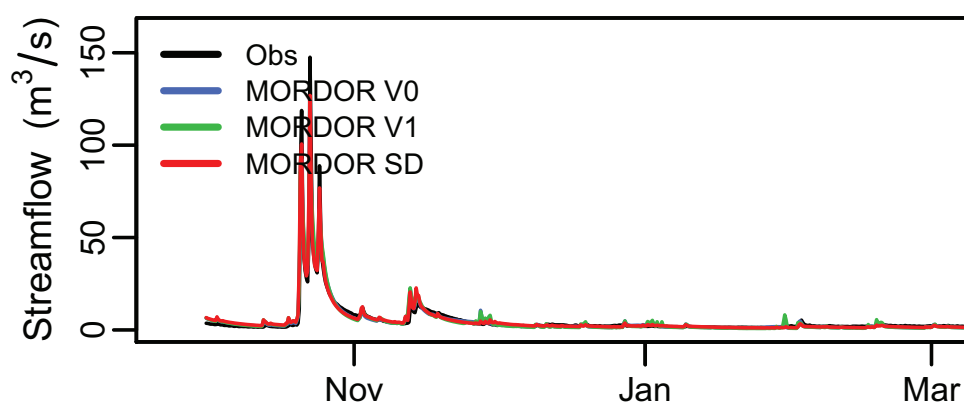

Mnde

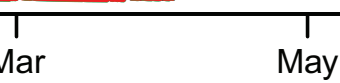

Year 2001

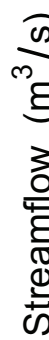

is

- $\longrightarrow$ MORDOR Vd

- MORDOR V
- MORDOR SD

.

.

Uu

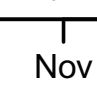

Nov
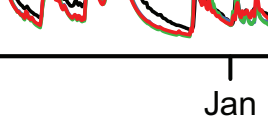

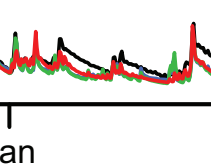

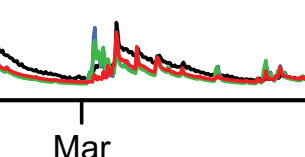

Year 2002
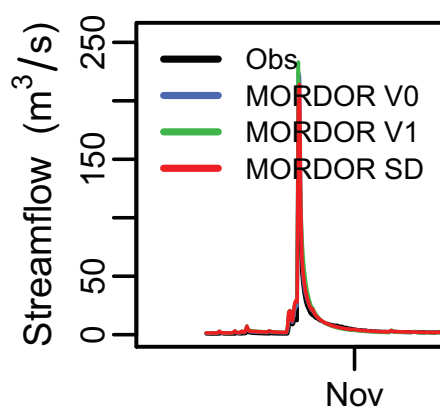

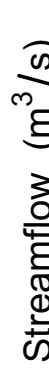

。

ov

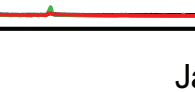

Jan

Mar

Year 2003

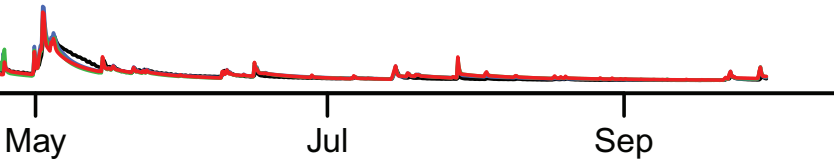

Year 2002 


\section{Lot@Castelnau}

Year 1989
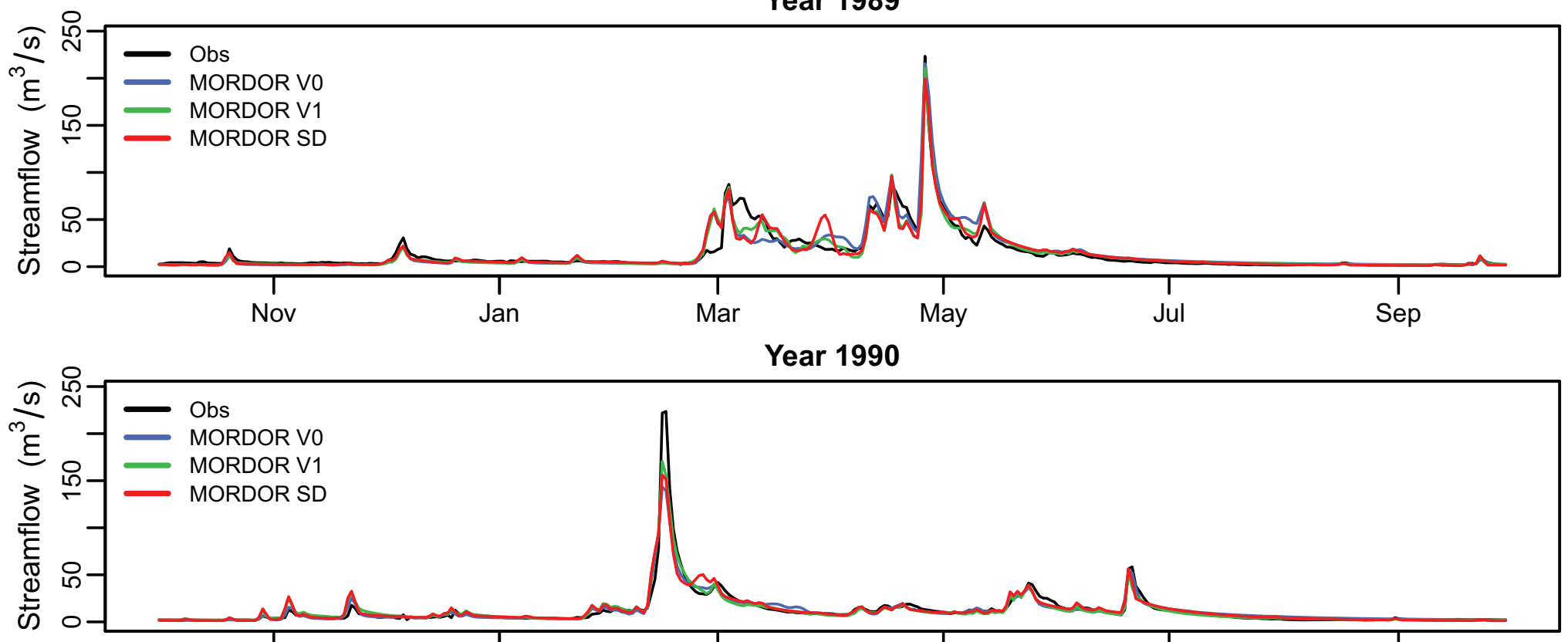

Year 1990

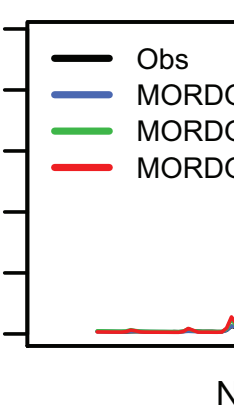

\section{R VO}

coso

A A san son

Nov

Jan

Year 1991

$\frac{0}{m}$
$\xi$
$\xi$
3
0
0
0
0

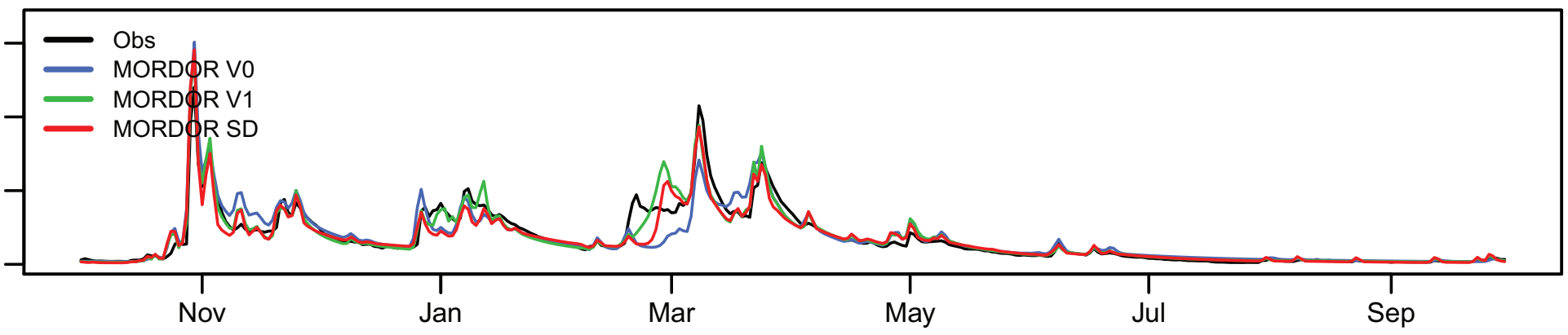

Year 1992
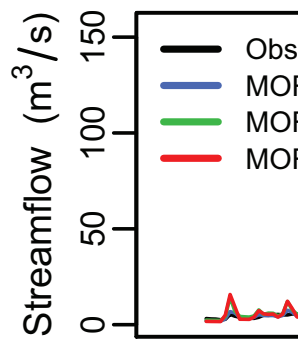

(10000

으-MORDOR V1

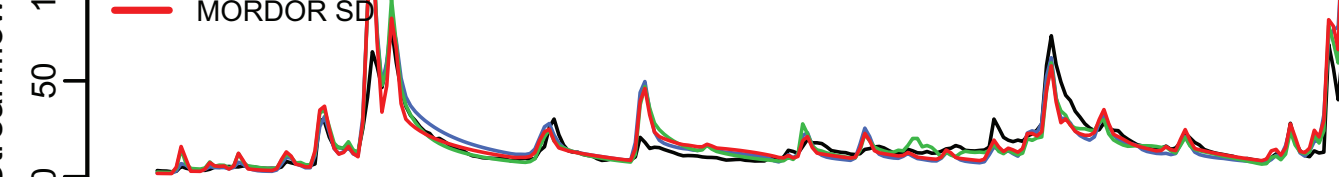

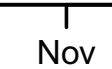

Jan

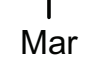

May

Year 1993

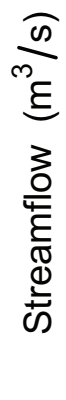

- obs
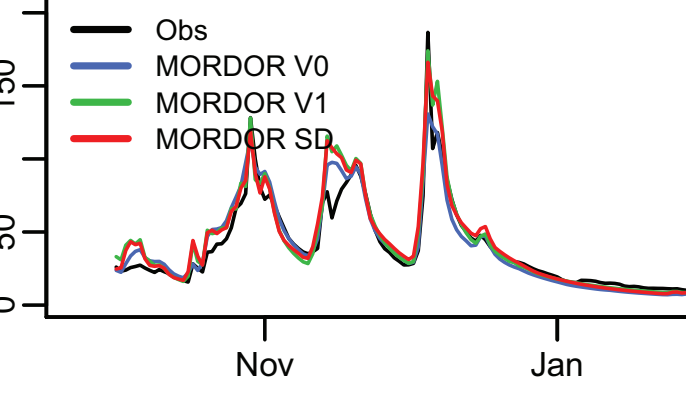

Jan
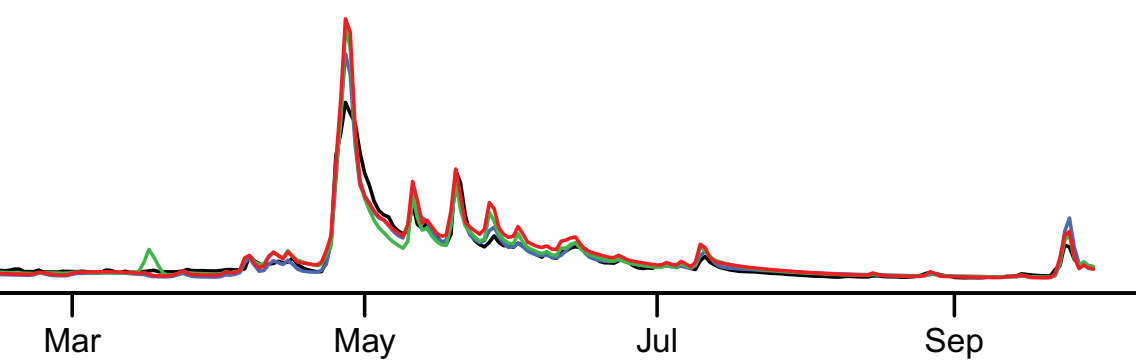

Year 1994
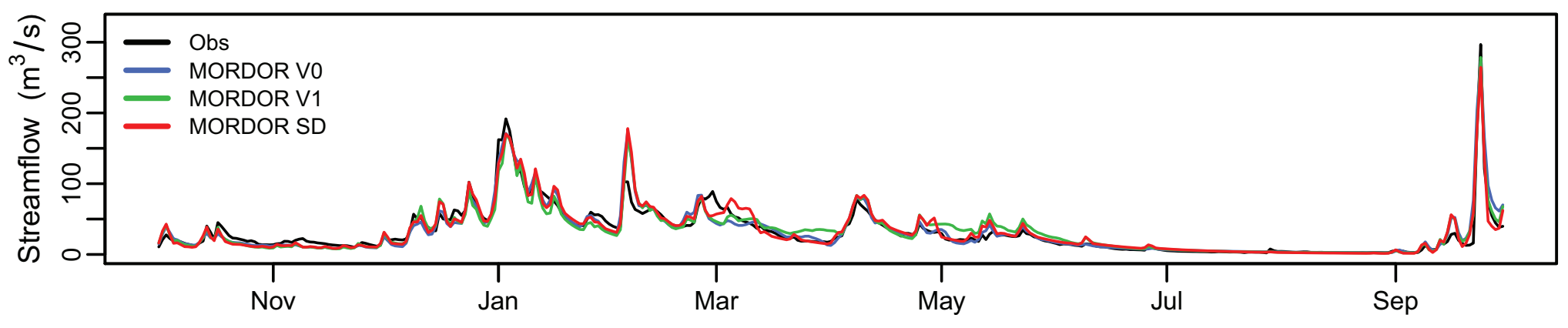


\section{Mimente@Florac}

Year 1986

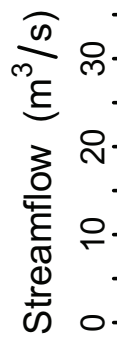

$\begin{array}{ll}- & \text { Obs } \\ \text { MORDOR V0 } & \text { MORDOR V1 }\end{array}$

$\longrightarrow$ MORDOR SD
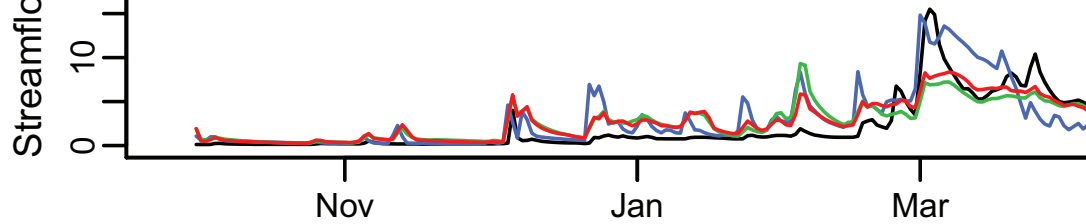

Mar

Year 1987
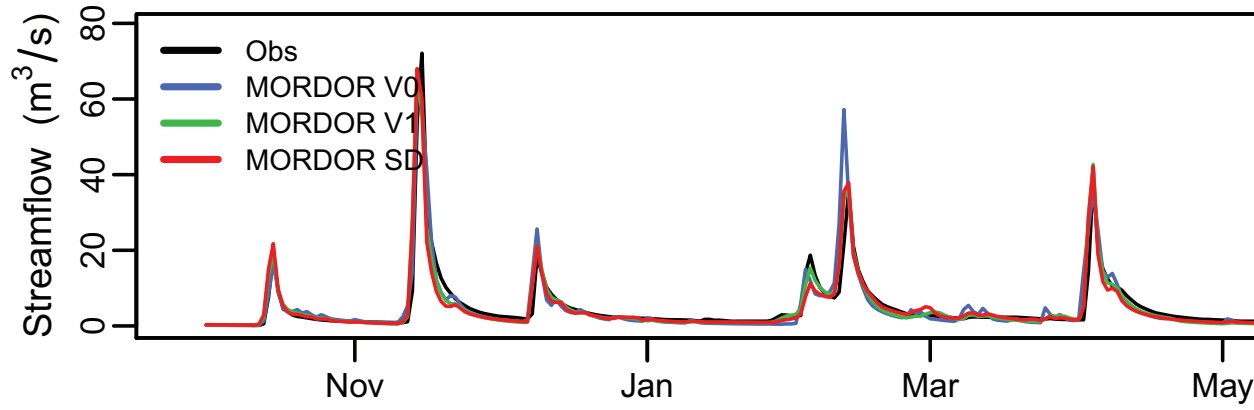

Year 1988
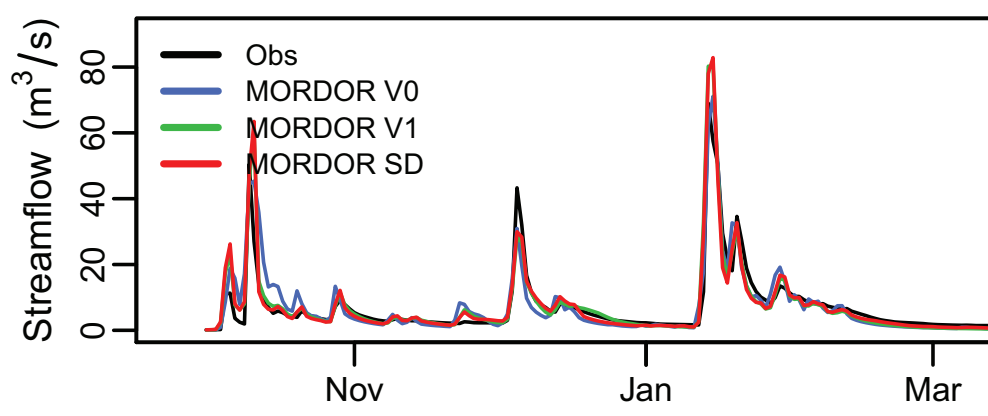

A. Donas

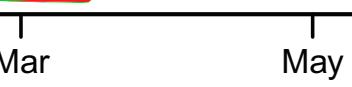

Year 1989
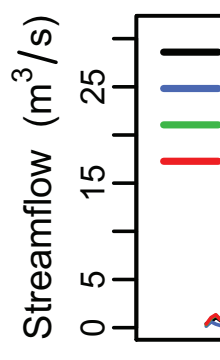

\section{Obs}

MORDOR V0

MORDOR V1

MORDOR SD

$\mathbb{N}$

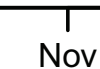

Nov

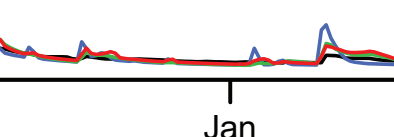

Jan

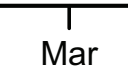

Year 1990

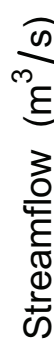

Obs

MORDOR V0

MORDOR V1

- MORDOR SD

으-

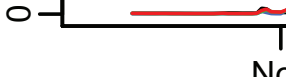

Nov
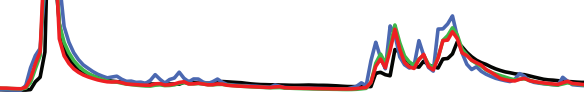

Jan

Mar

Year 1991

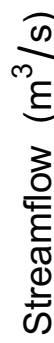

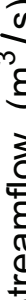

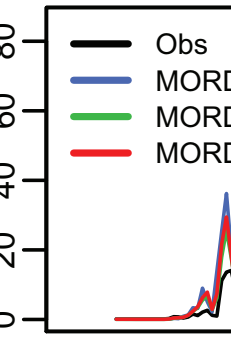


Mimente@Florac

Year 2004
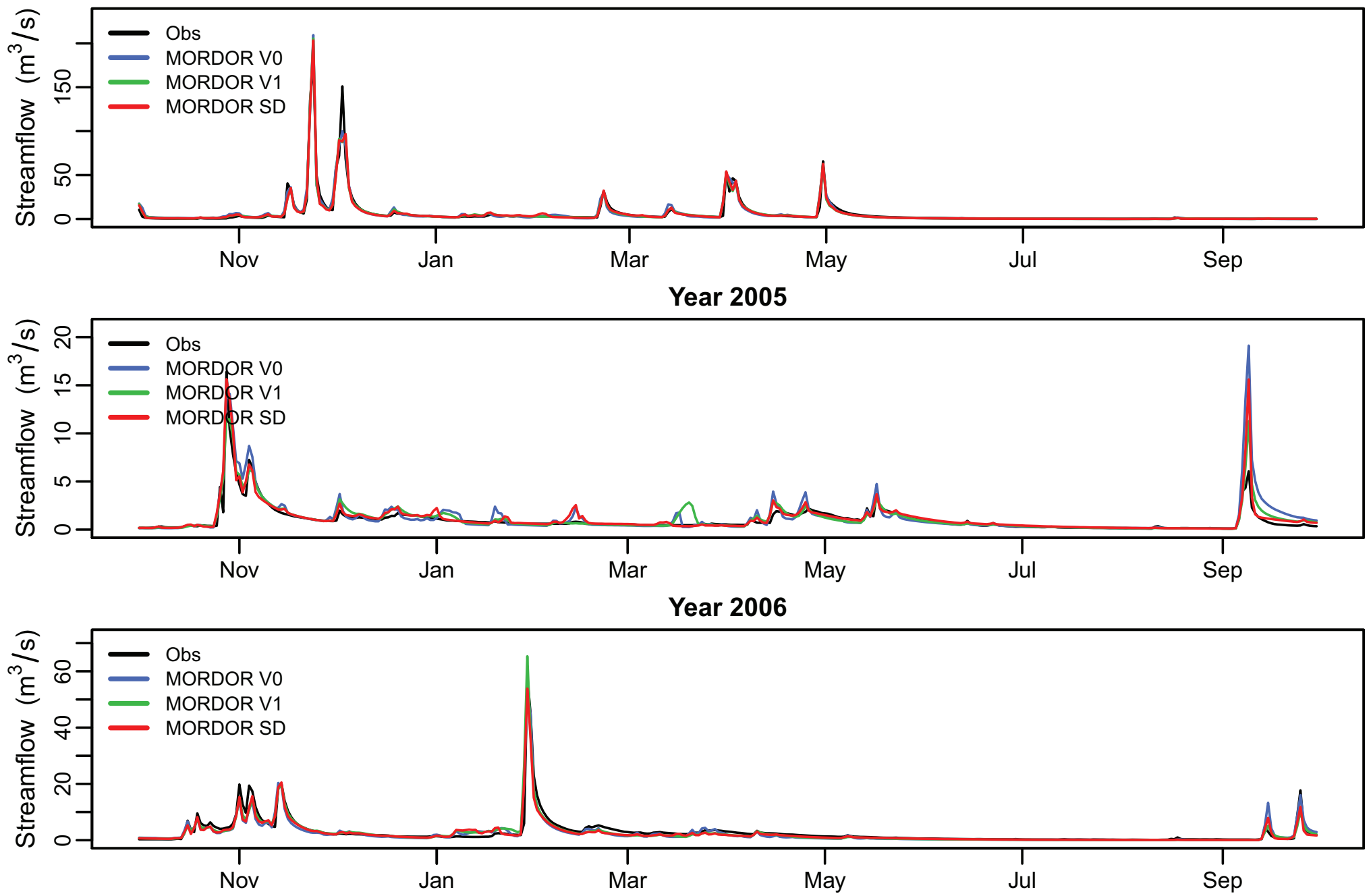
Montane@Eyrein

Year 1959

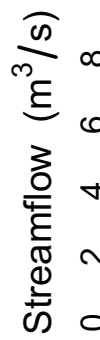

Obs

- MORDOR VO

- MORDOR V

MORDOR SD

then

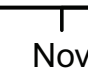

Nov

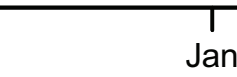

Jan

WNa

Non

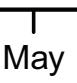

und

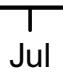

Sep

Year 1960

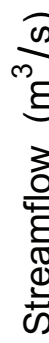

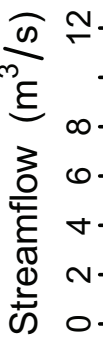

- Obs

- MORDOR V0

- MORDOR V1

- MORDOR SD

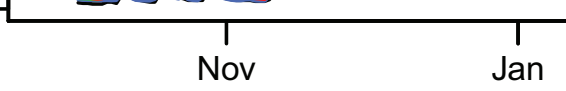

Jan Mar

Year 1961

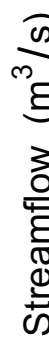

Obs

MORDOR V0

MORDOR V1

MORDOR SD

of of whan

o-

Nov

Year 1962

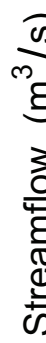

- Obs

MORDOR V0

으- MORDOR V1

MORDOR SD

$\underbrace{}_{\text {Nov }} \operatorname{Jan}_{\text {Mar }}^{N}$

Year 1963

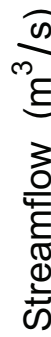

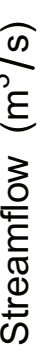

- Obs

Obs
MORDOR V0
MORDOR V1

MORDOR V1

$-$

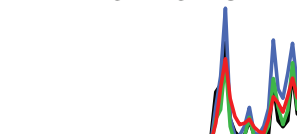

Ma

Nov
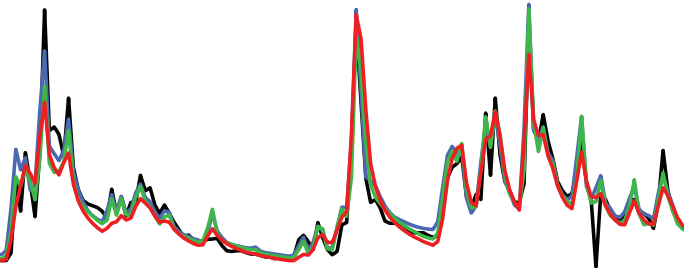

husphtoph

Jan

Mar

May

Jul

Sep

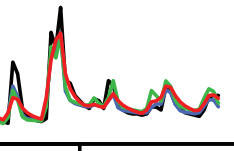

Sep 
Montane@Eyrein

Year 1971

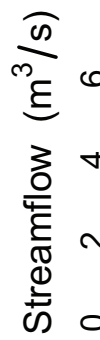

- Obs
MORDOR VO

$\longrightarrow$ MORDOR V1

$\checkmark-\longrightarrow$ MORDOR SD

h

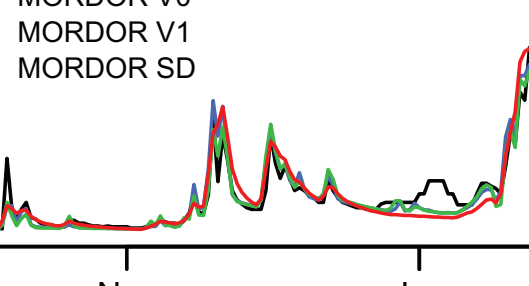

Nov

Jan

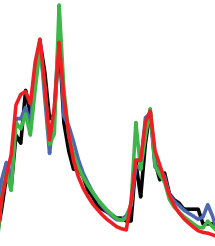

Mnum wasth

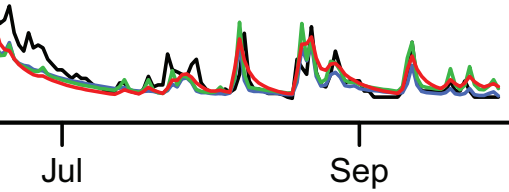

Year 1972

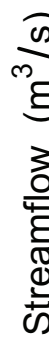

0
0
5
5
3
0
$\frac{0}{4}$
0
0
0
0

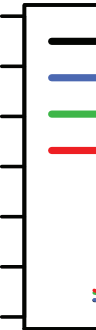

Obs

- MORDOR VO

- MORDOR V1

MORDOR SD

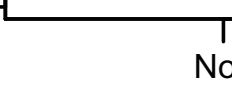

Nov

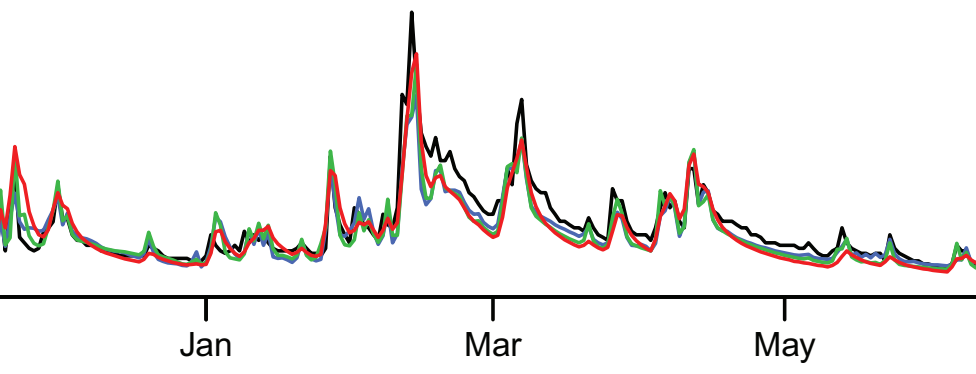

Year 1973

$\frac{\pi}{m}$
m
3
0
$\overline{0}$
$\overline{0}$
0
0
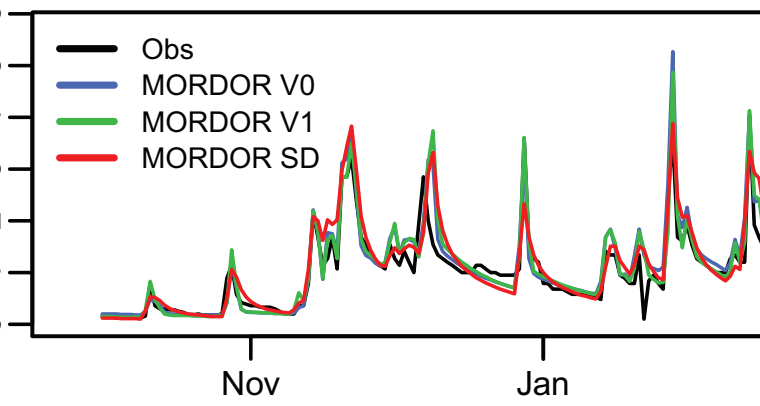

Mamal

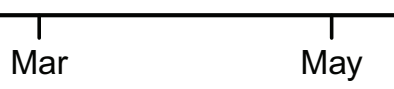

Year 1974
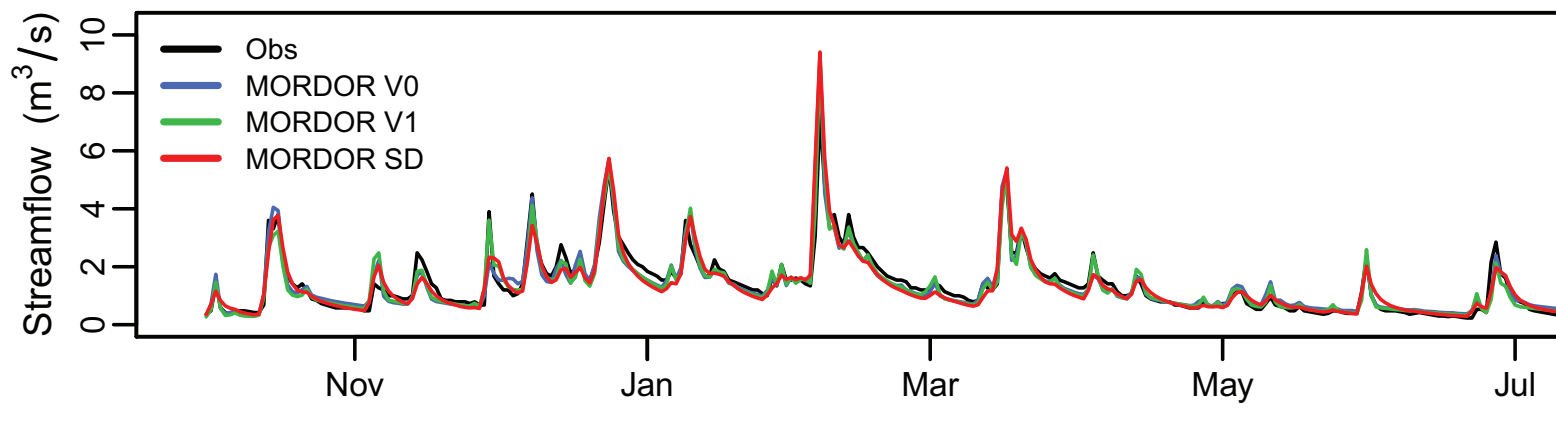

Year 1975
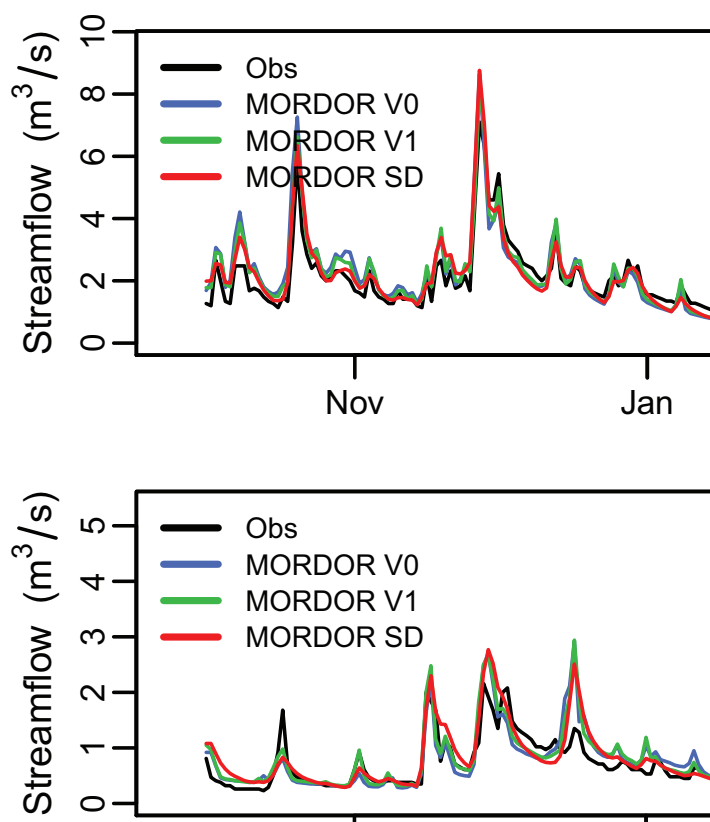

Nov

Jan

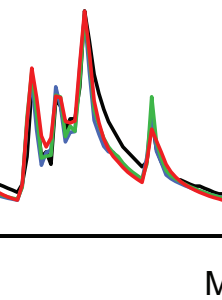

Mothonula

Mar

Year 1976

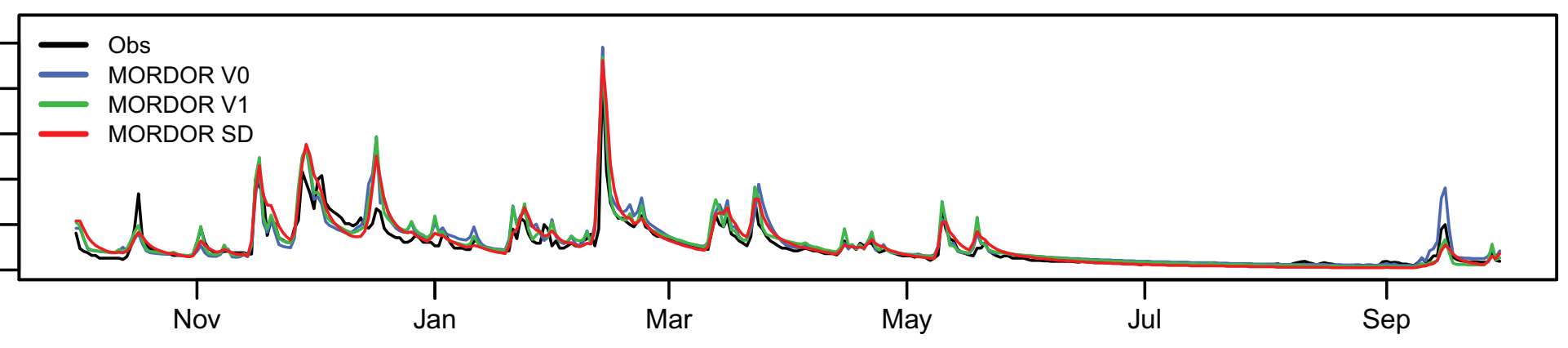


Montane@Eyrein

Year 1989

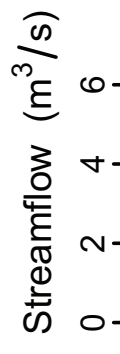

- Obs

MORDOR V0

MORDOR V1

MORDOR SD

uncoluenar

Nov

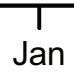

Year 1990

$\frac{\pi}{0}$
ल
5
3
0
5
5
0
0
0

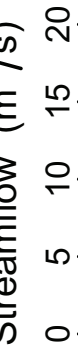

Obs

MORDOR V0

MORDOR V1

MORDOR SD

Nov

Nov

ind

Jan Mar

Year 1991

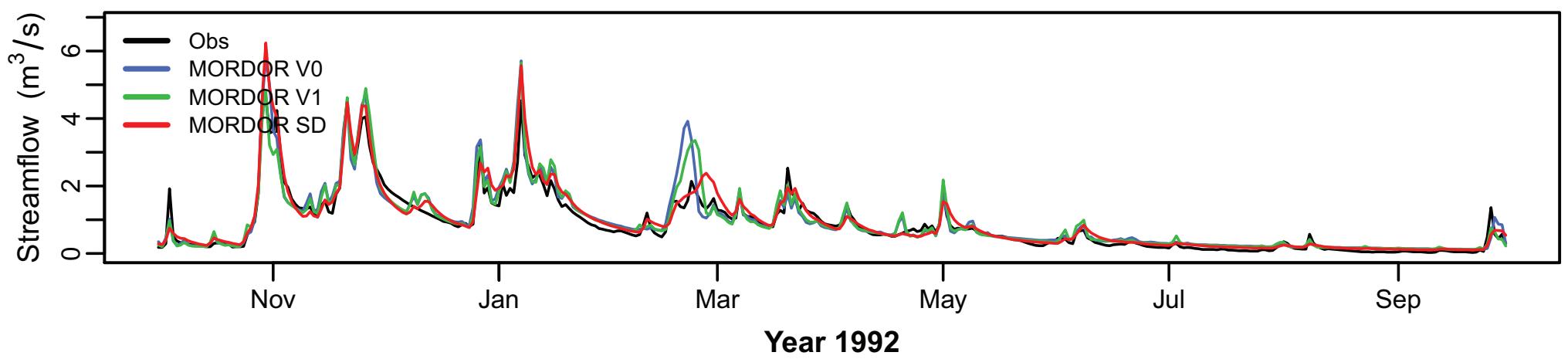

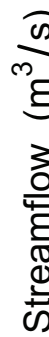

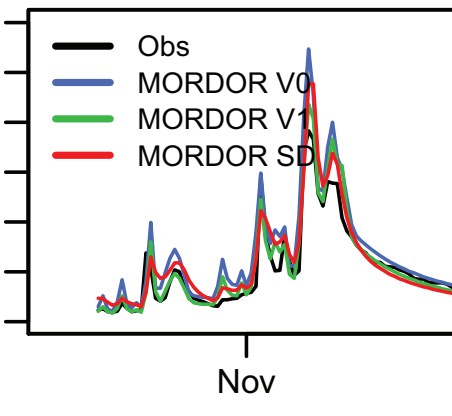

(1)

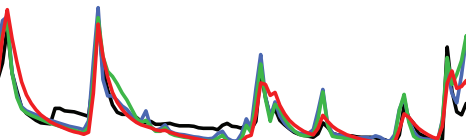

the

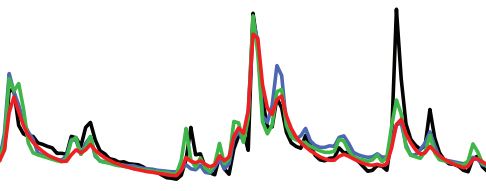

$\underset{\text { Jan }}{1}$

Mar

May

Jul

Year 1993

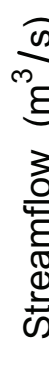
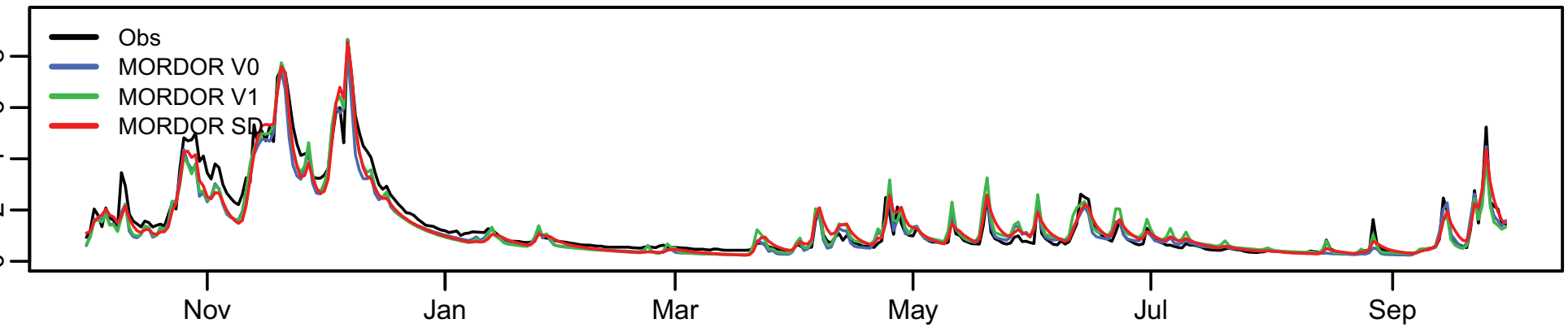

Year 1994
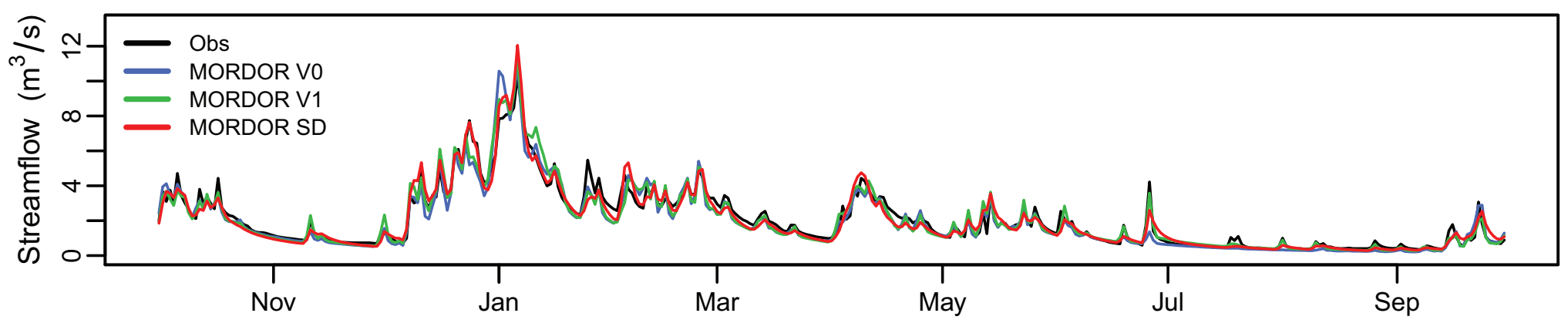
Montane@Eyrein

Year 1995

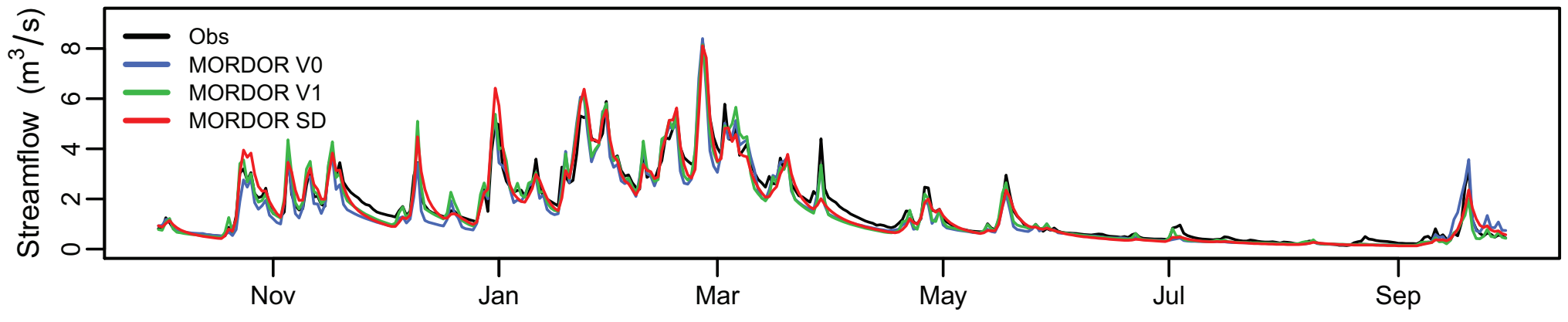

Year 1996

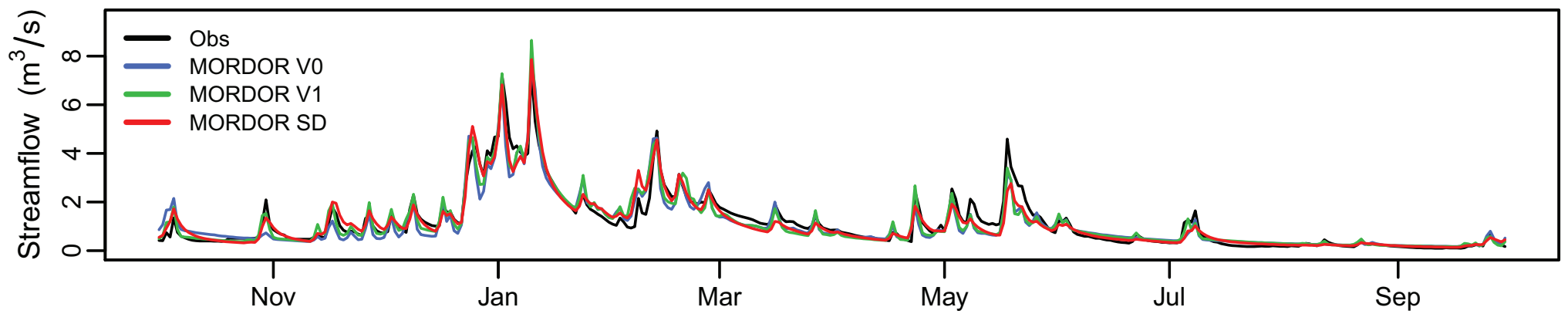

Year 1997
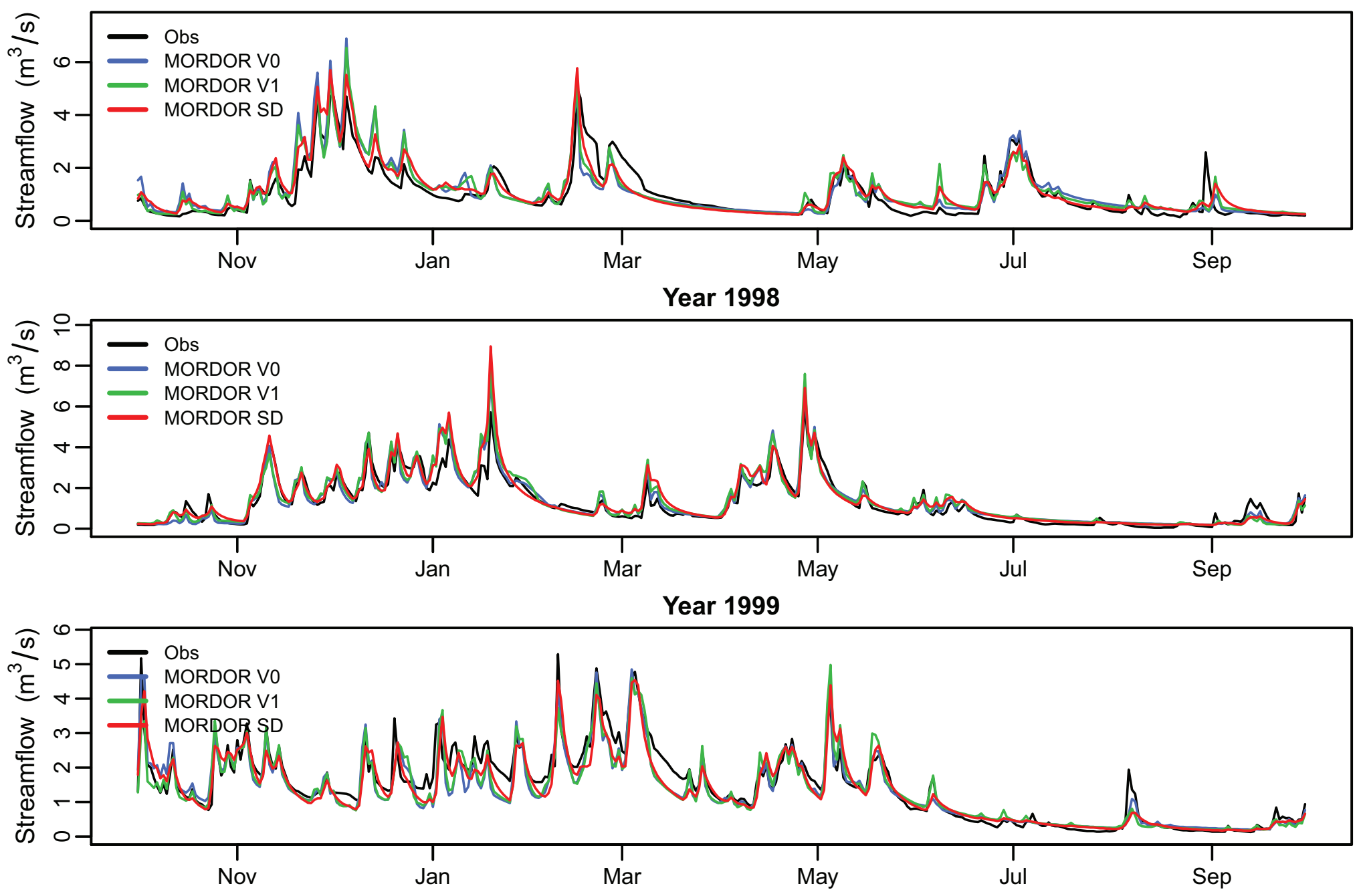


\section{Oriege@Campauleil}

\section{Year 1961}

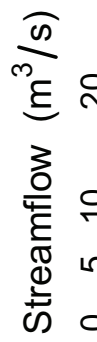

相

Obs

MORDOR V0

MORDOR V1

MORDOR SD

$\ln 1$

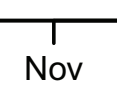

A A Amantons and ondm

Nov

Jan

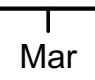

May

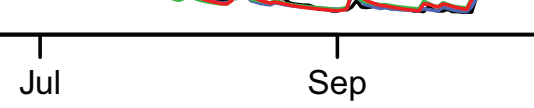

Year 1962

क

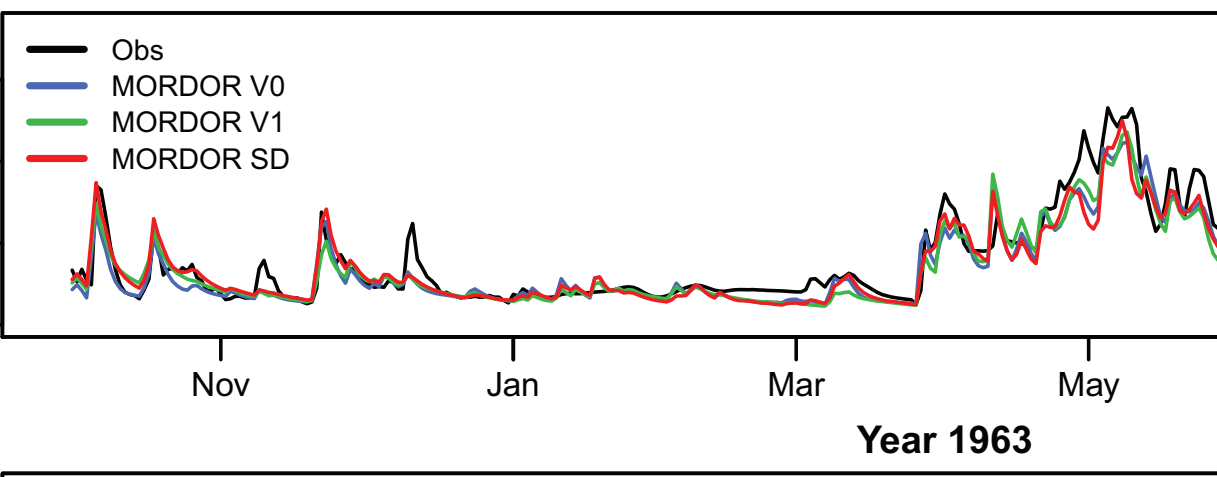

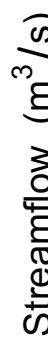

$-\begin{array}{ll}- & \text { Obs } \\ - & \text { MORDOR V0 } \\ \text { MORDOR V1 }\end{array}$

- MORDOR V1

MR

으-

Nov

$M$

Nov

Ja

Jan Mar

Mar May

Year 1964

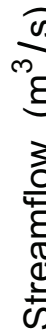

- Obs

MORDOR VO

- MORDOR SD

(n)

唝 $-$

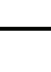

Nov

$\sim N$

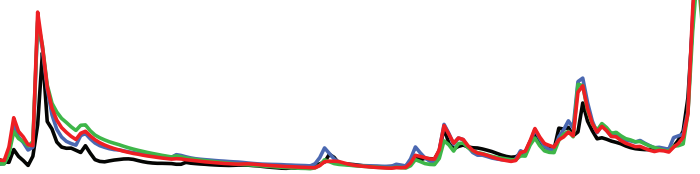

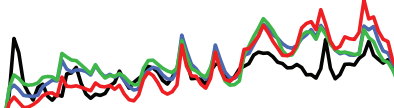
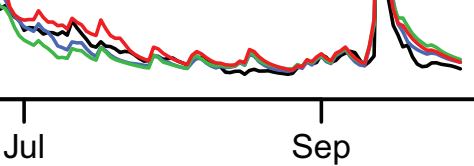

Sep

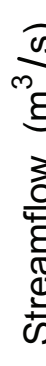

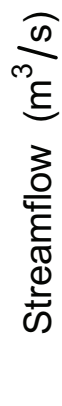

$-$

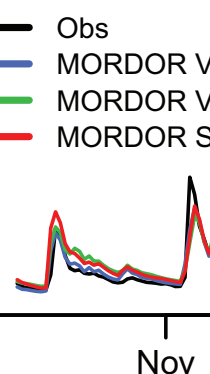

\section{vo}

SD

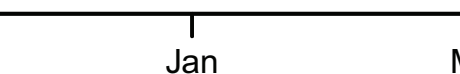

Mar

\section{Year 1965}

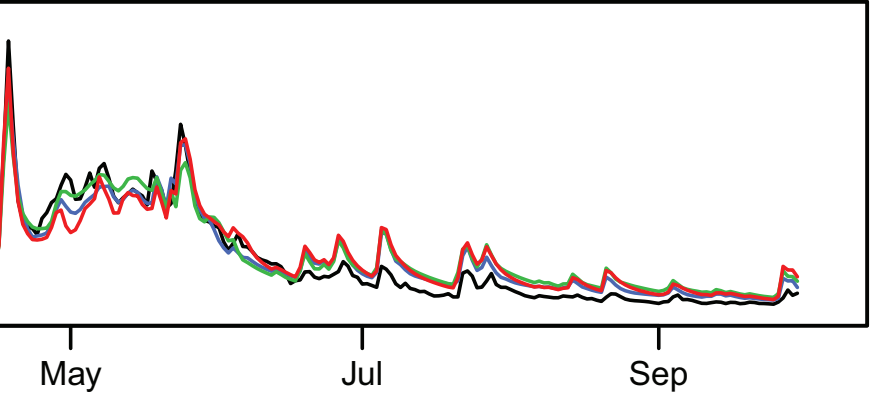


Romanche@Chambon

Year 2004

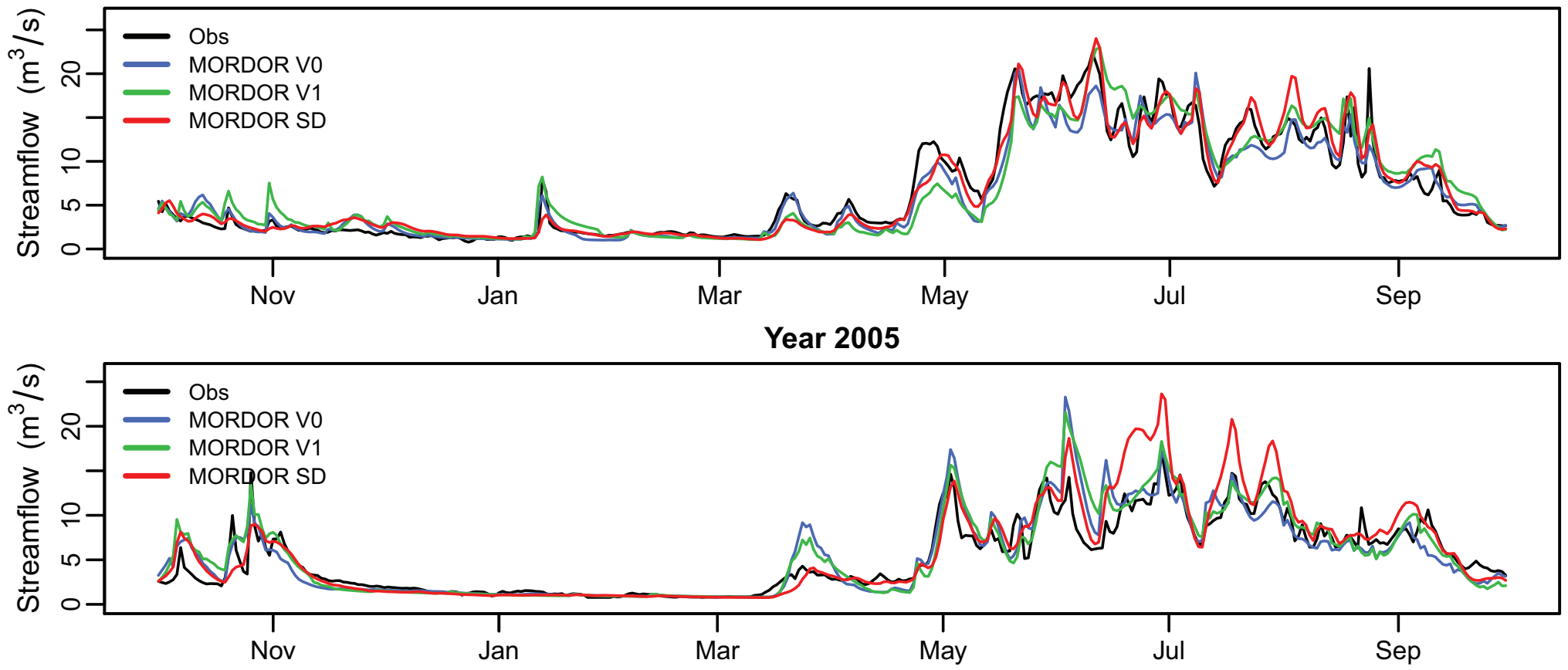



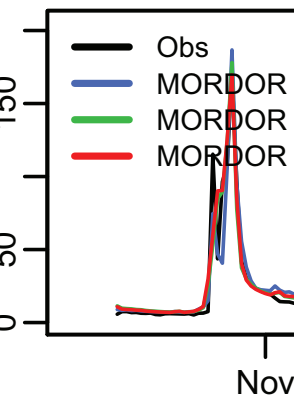

Year 2001

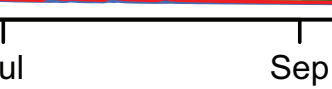

क 8

- Obs

- MORDOR VO

- MORDOR/VI

- MORDOR SL

-7 NWW

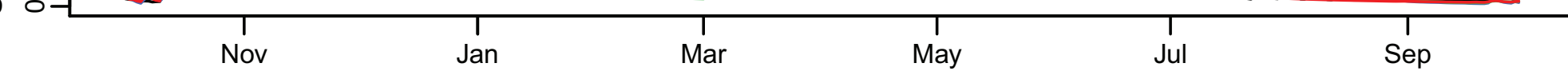

Year 2002

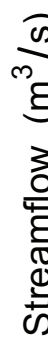

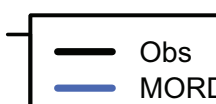

○- MORDOR V0

MORDOR SD

sontmons

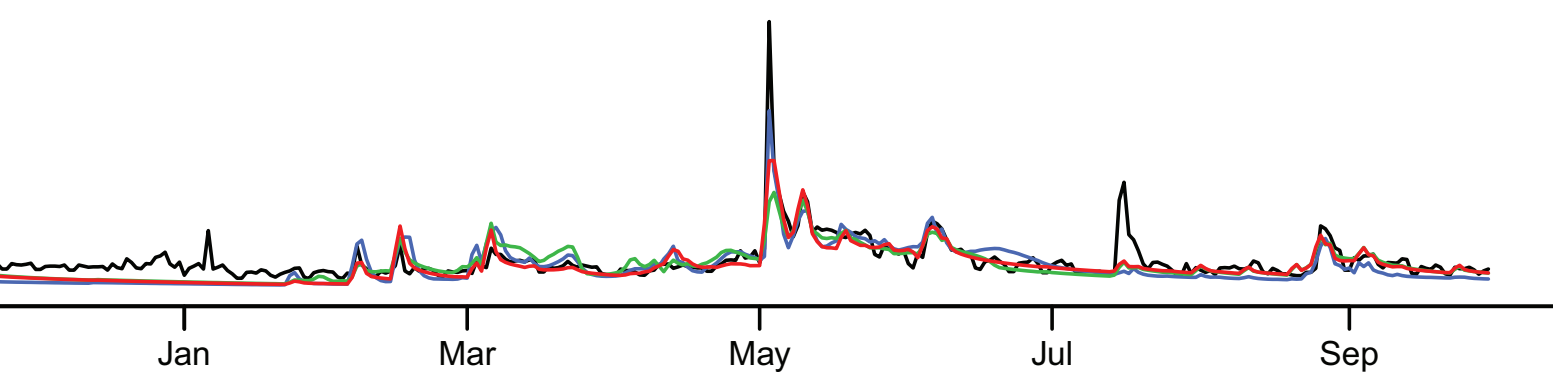

Year 2003
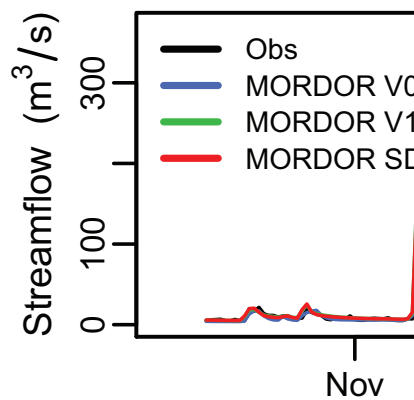

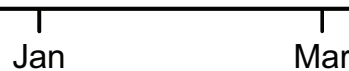

Mar

Year 2004

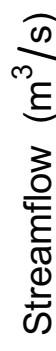

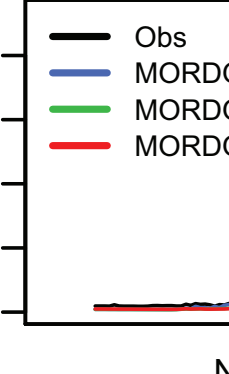

$$
\text { Nov }
$$

A

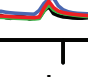

Jan

Mar

Year 2005
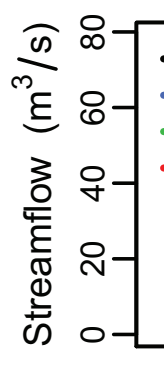

- Obs

- MORDOR V0

- MORDOR V1

- MORDQR SD

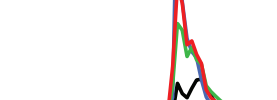

$\sim$

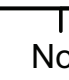

Nov

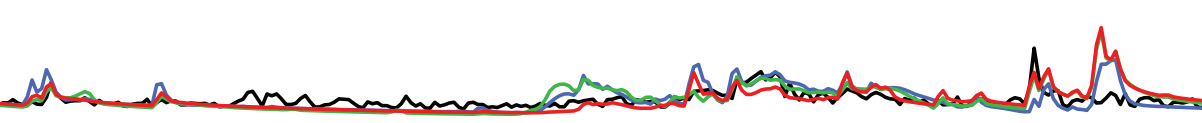

- 
Souloise@Infernet

Year 1992

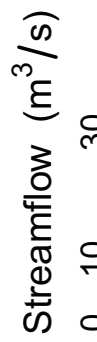

相

Obs

MORDOR V0

MORDOR V1

A MRDOR SD

Wa

Nov

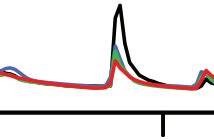

Jan Mar

Mar May

Year 1993

क

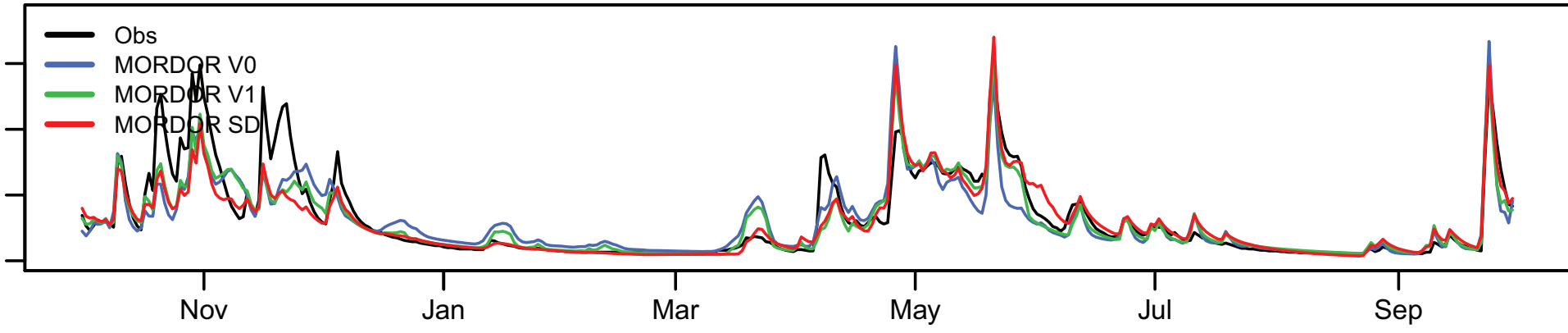

Year 1994

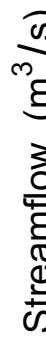

$--{ }_{-}^{M}$

Obs

T

MORDOR V1

ORDOR SD

Nov

an Mar

Nan Nana

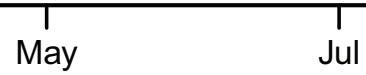

NA

Year 1995

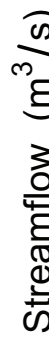

- Obs

- MORDOR VO

- MORDOR V1
- MORDOR SD

$-\mathrm{N}$

Nov

Nov

A

and

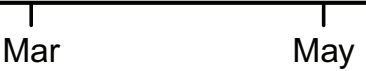

an

Jul

Year 1996

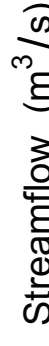

- Obs

$\begin{array}{ll}- & \text { Obs } \\ & \text { MORDOR V0 } \\ \text { MORDOR V1 }\end{array}$

$-\begin{aligned} & \text { MORDOR V1 } \\ & -\quad \text { MORDOR SD }\end{aligned}$
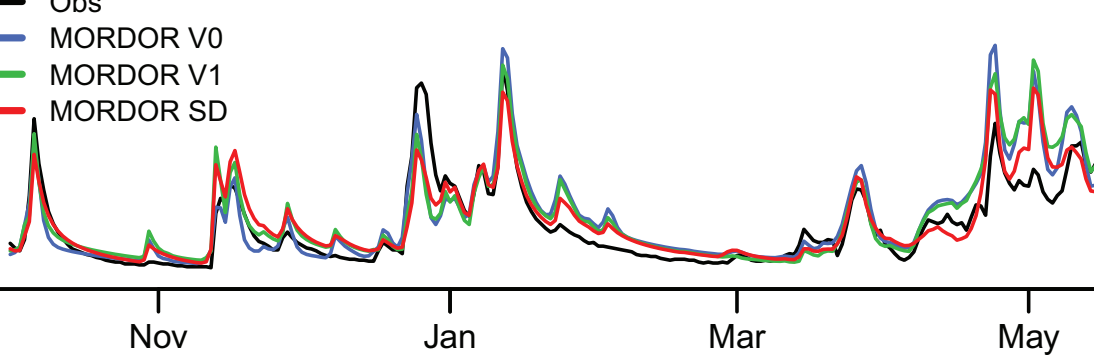

tyons

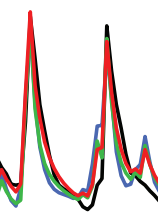

Sep

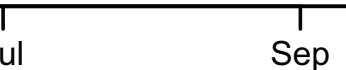

Year 1997

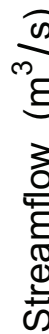

- Obs

$\infty$
$8-1$
$o-1$
$\circ-1$
$\circ-1$

- MOR

- MORDOR V

- MORDOR SB 

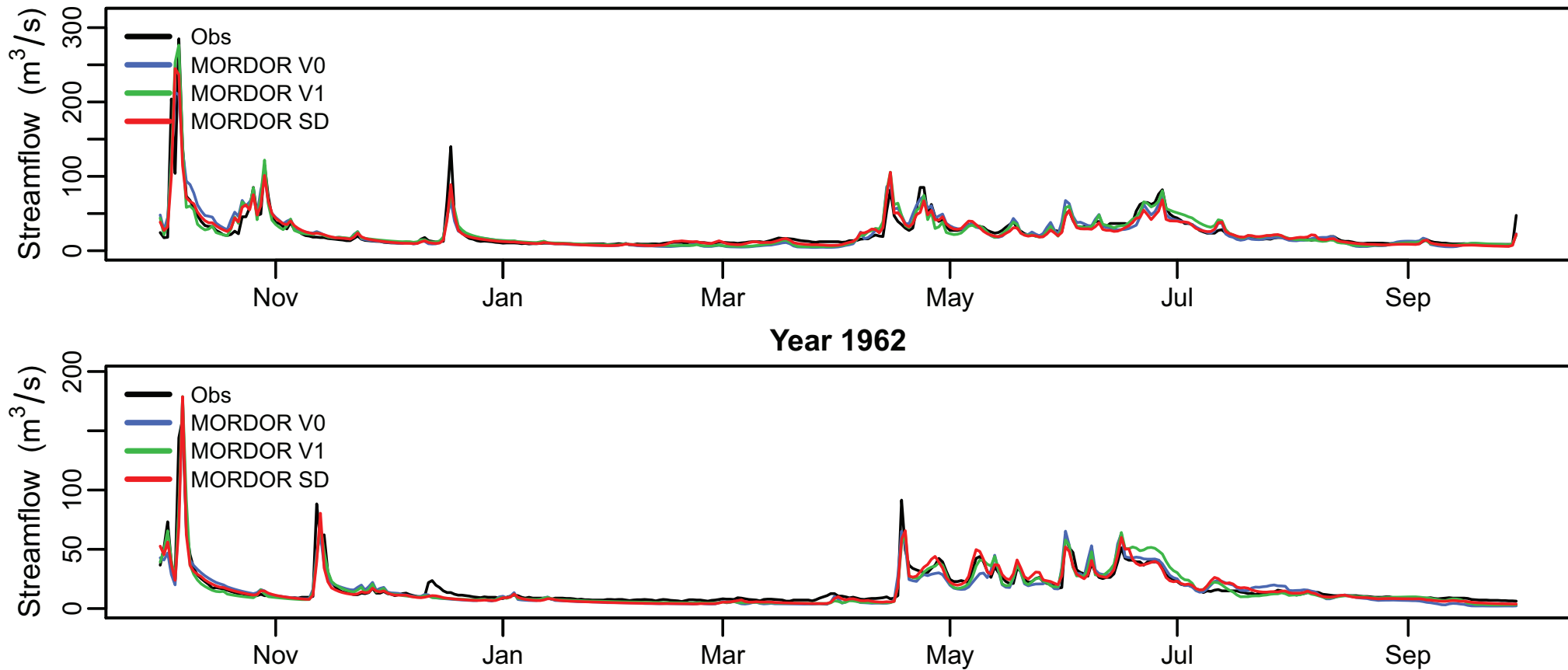

Year 1962

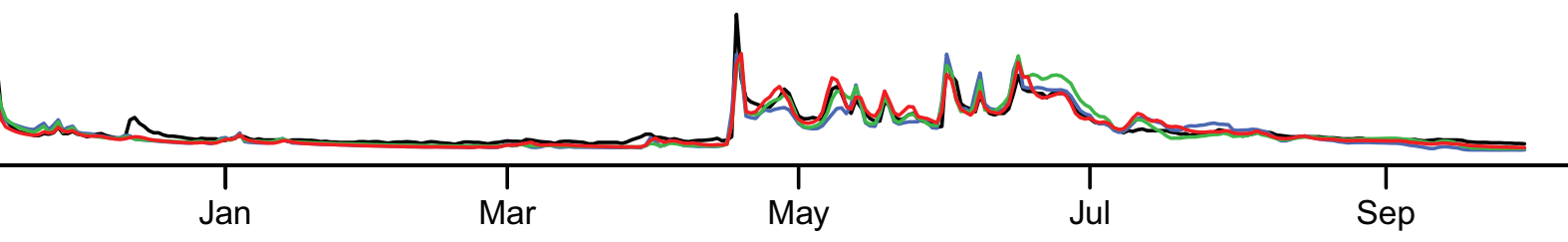

Year 1963

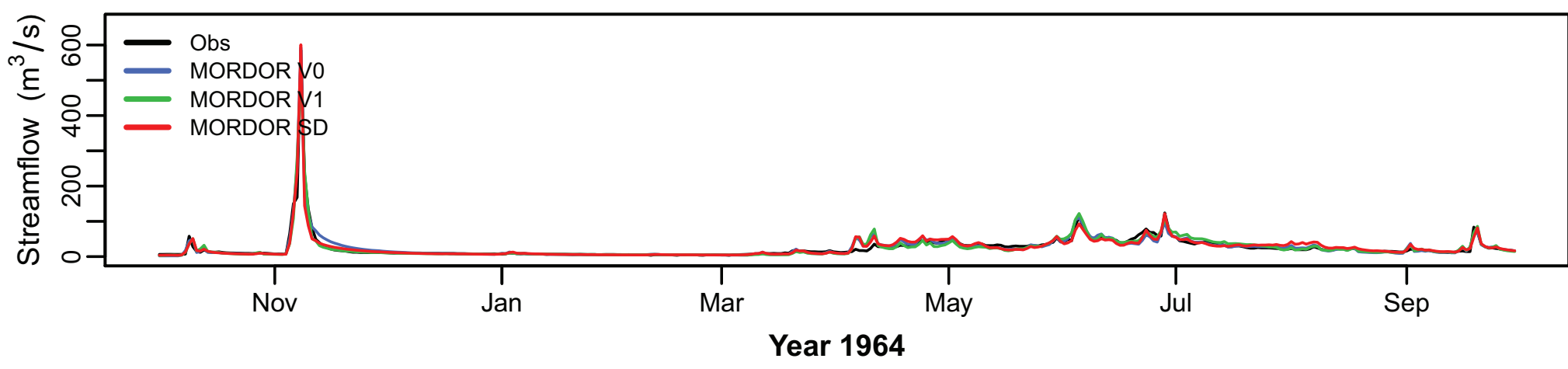

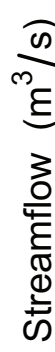

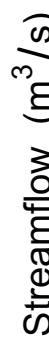

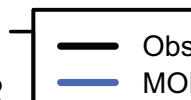

MORDOR V0

MORDOR V1

MORDOR SD
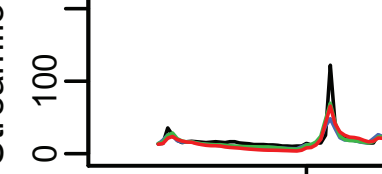

Nov

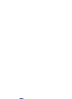

$\Lambda N$

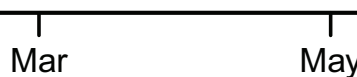

Sep

Year 1965

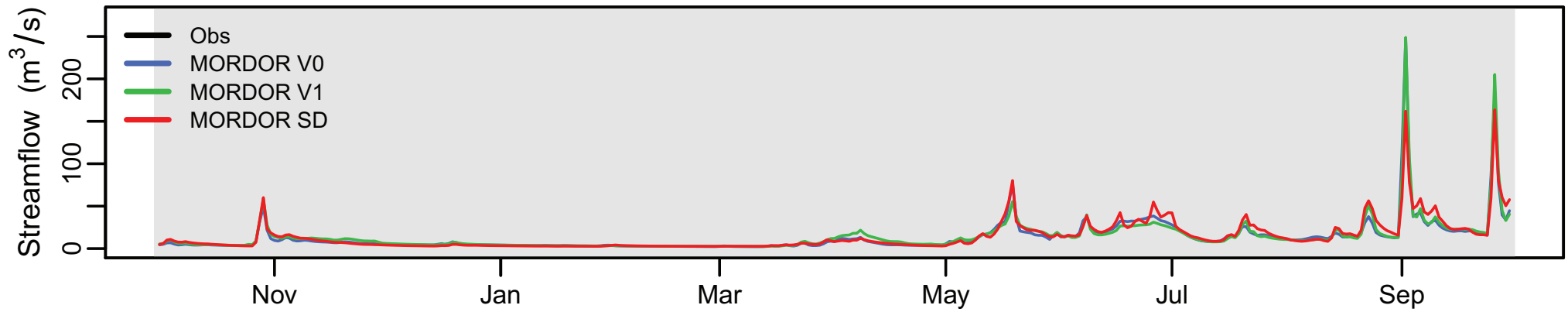

Year 1966
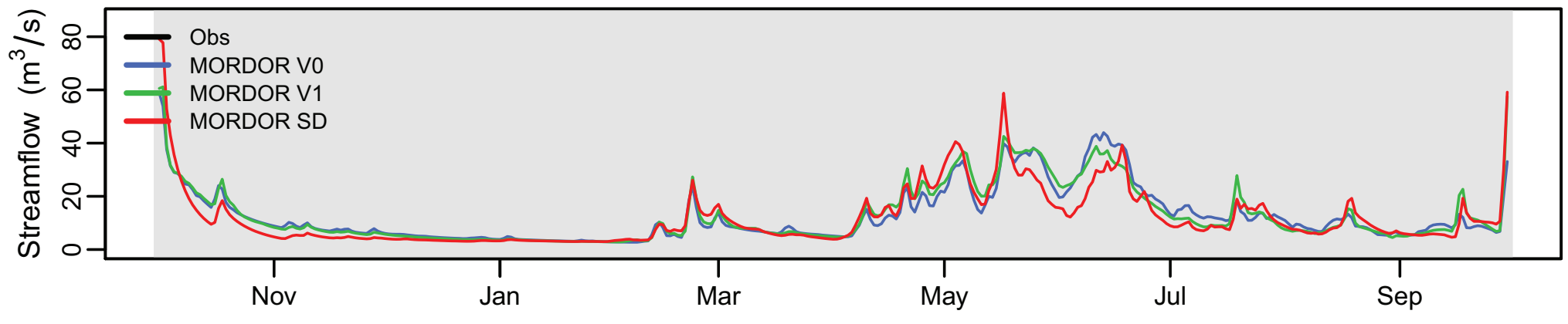

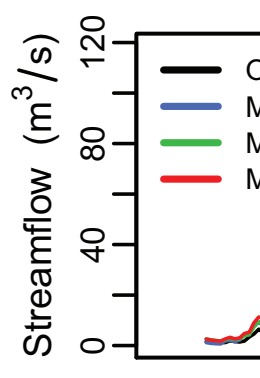

Obs

MORDOR VO

MORDOR V1

के

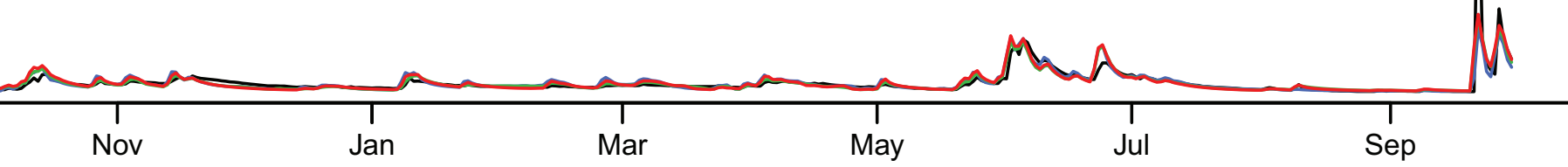

Year 1993

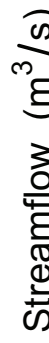

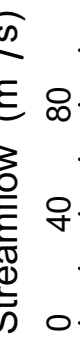

- Obs

- MORDOR V0

- MORDOR V1

MORDOR SD

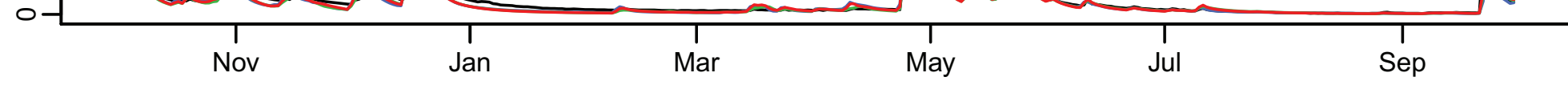

Year 1994

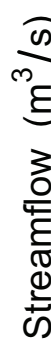

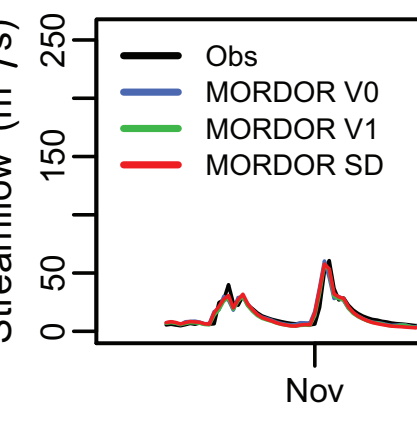

co

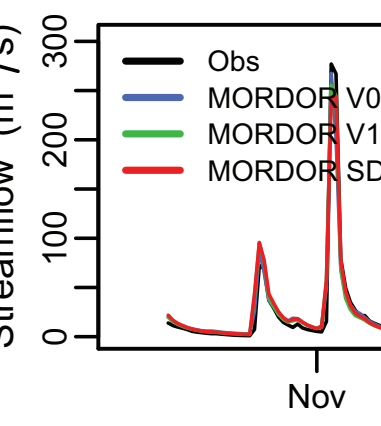

$$
\frac{0}{0}
$$

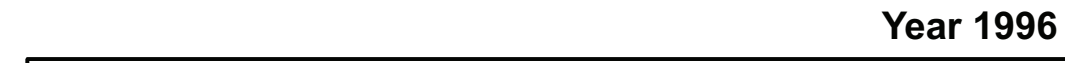

Year 1996

Year 1995

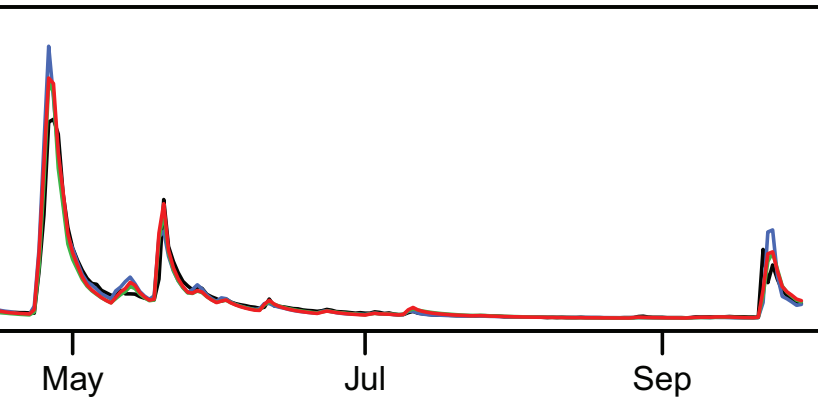




\section{Tarn@Millau}

Year 1993

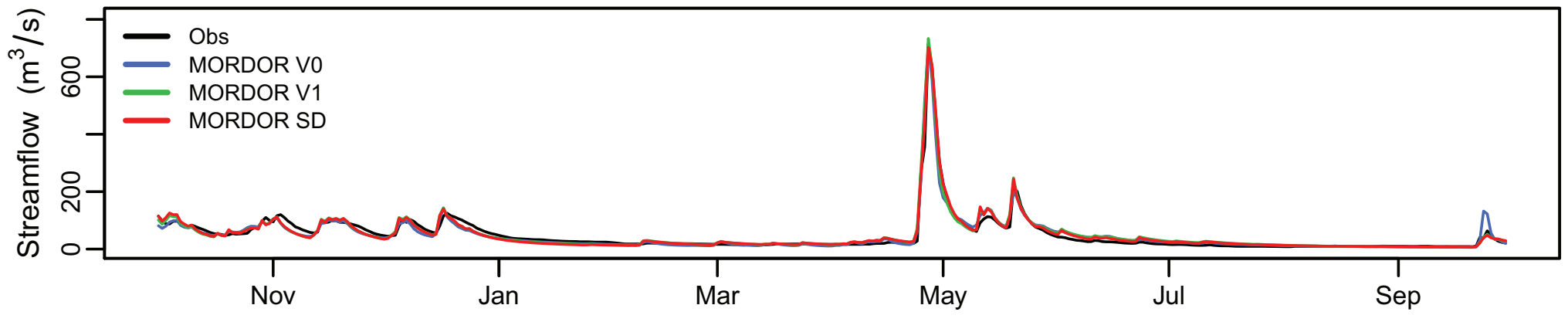

Year 1994
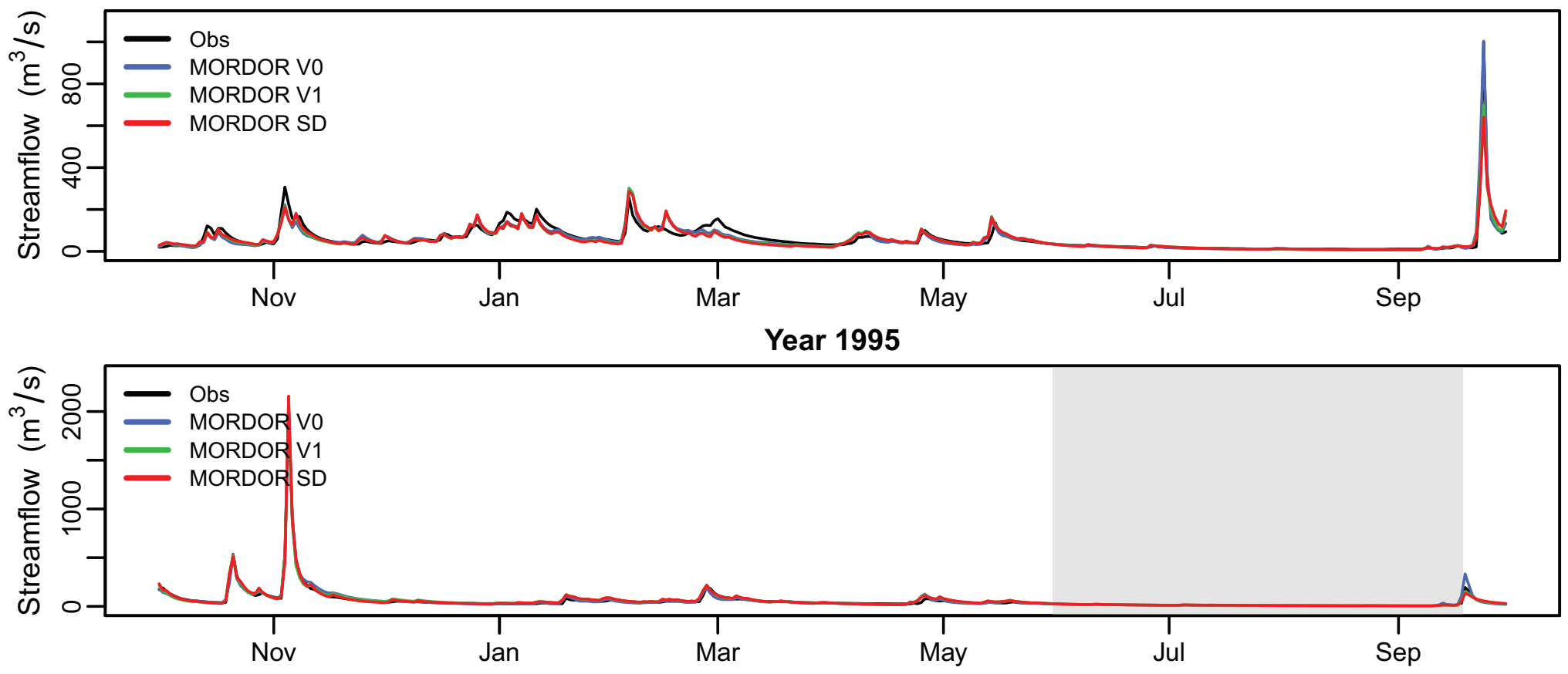

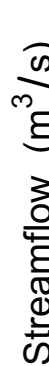

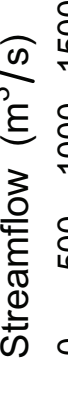

Year 1996

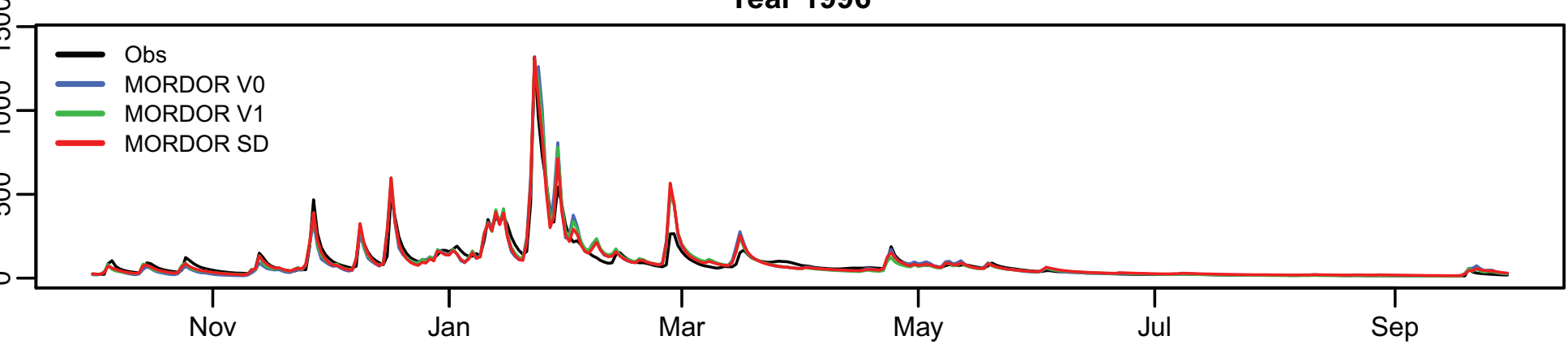

Year 1997
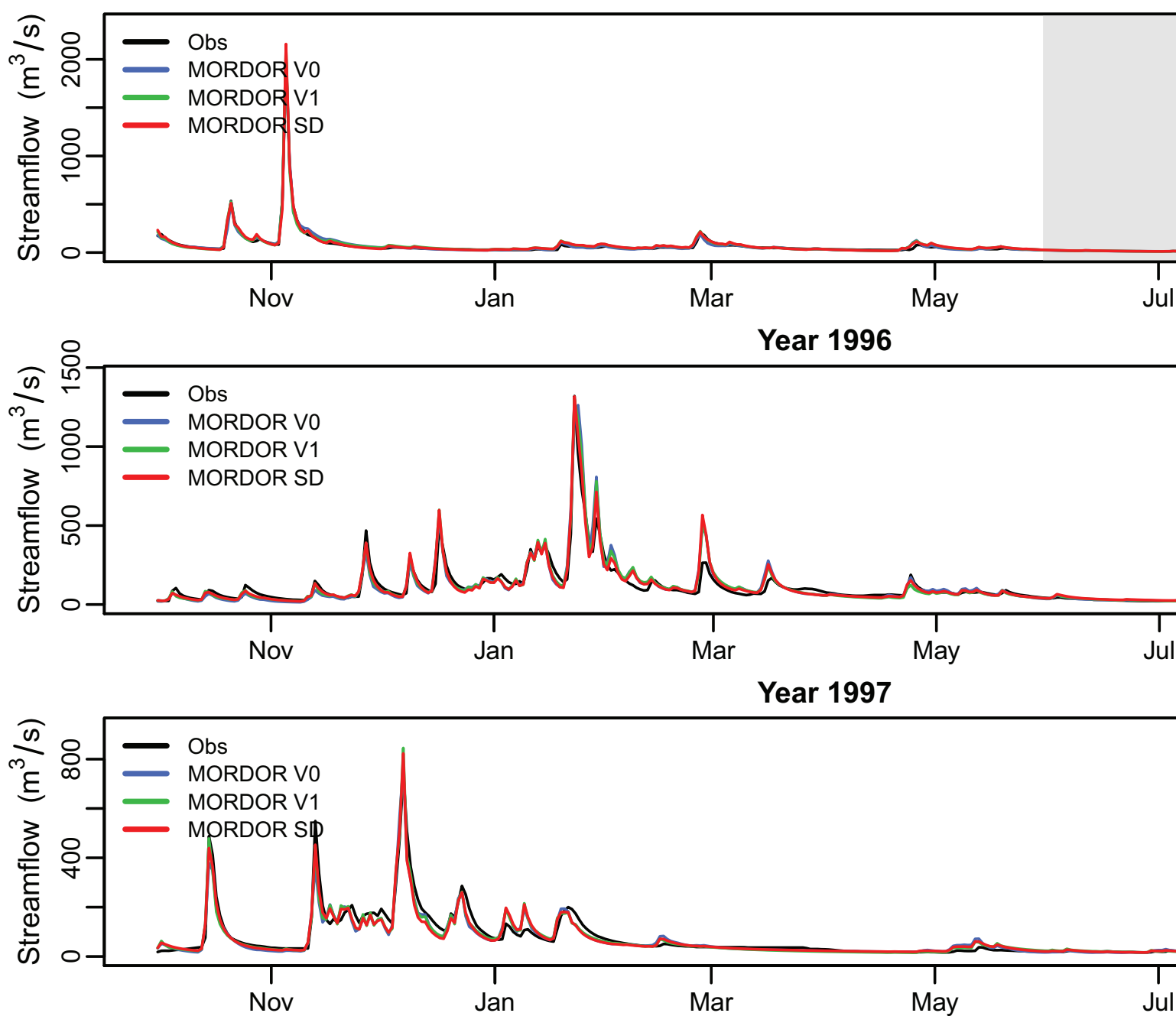

Sep

Year 1998
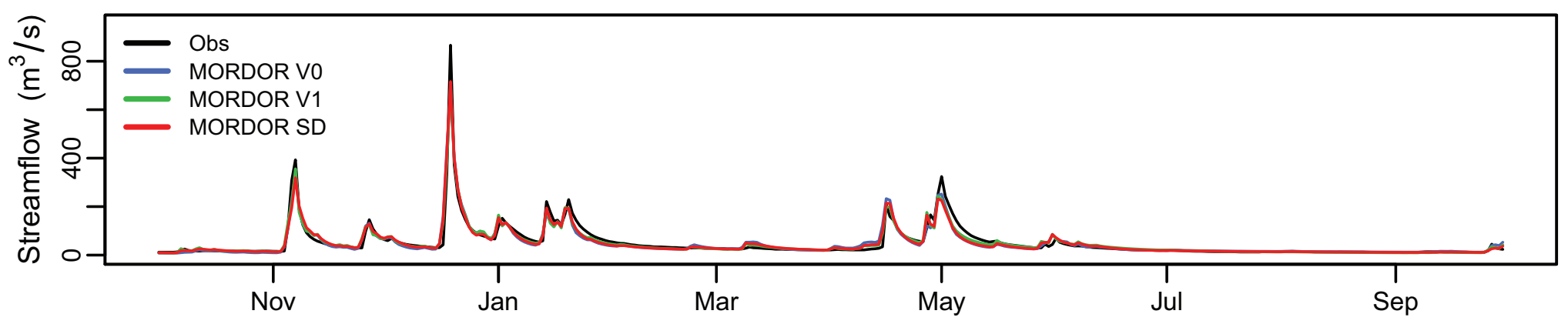


\section{Tarn@Millau}

Year 2005

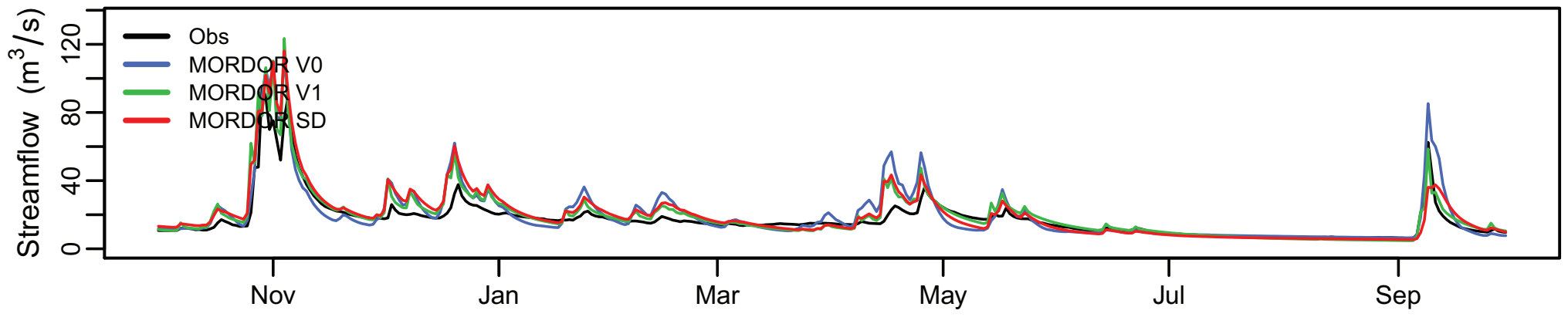

Year 2006
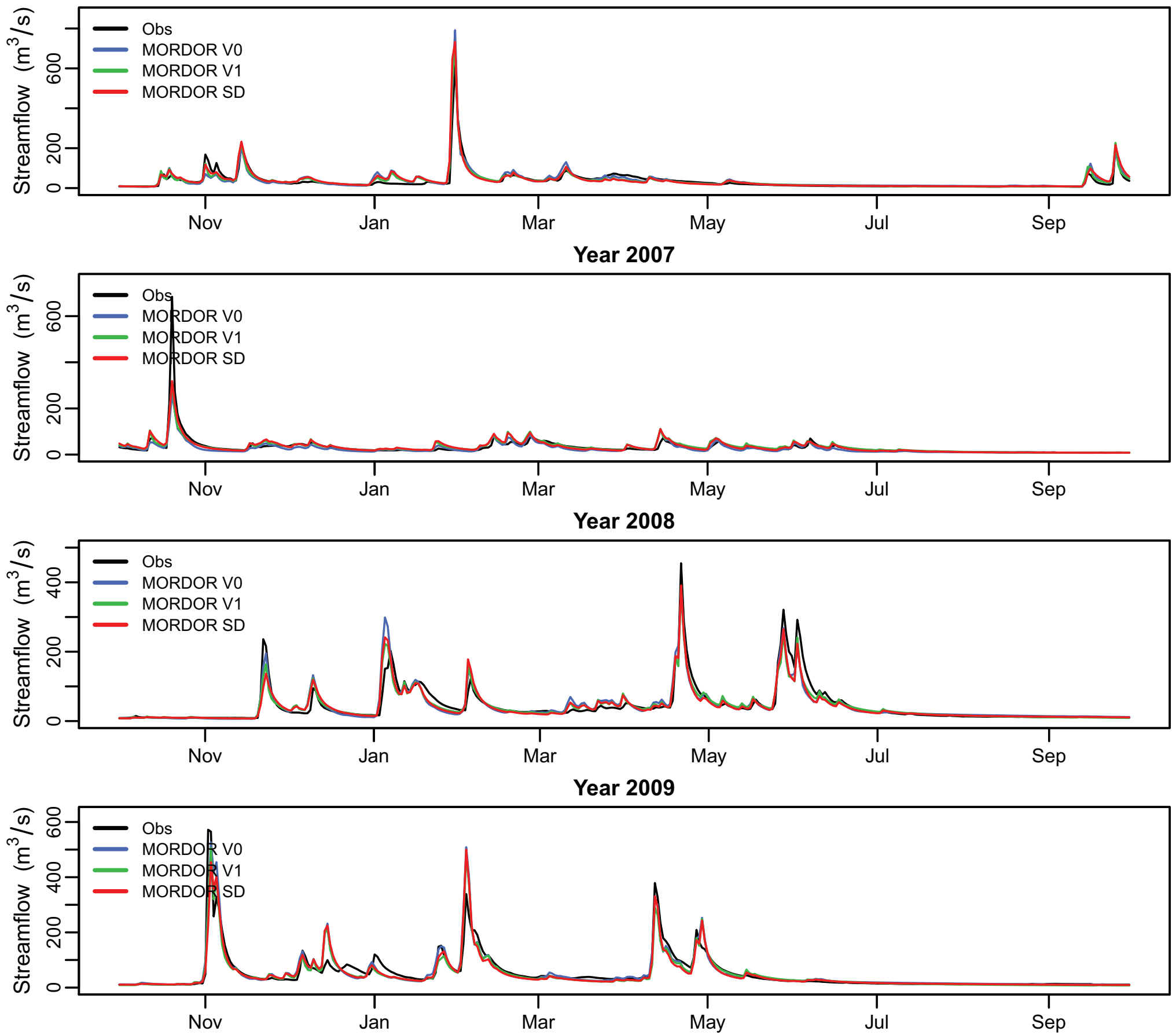

Year 2010

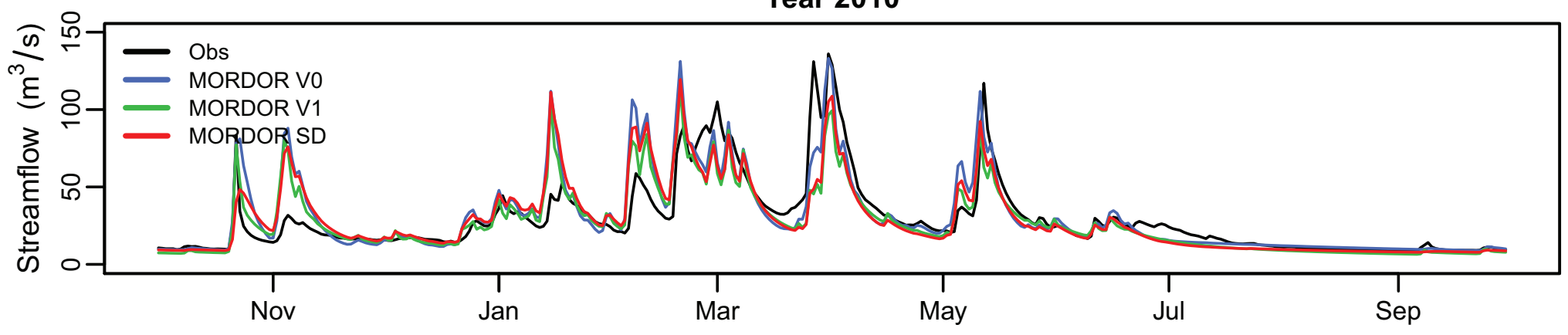




\section{Tarn@Montbrun}

\section{Year 1986}
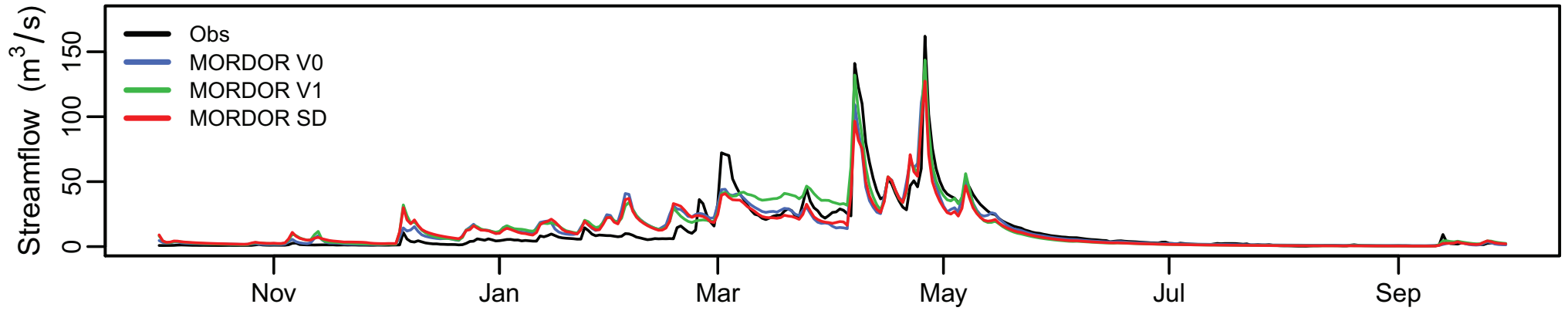

Year 1987
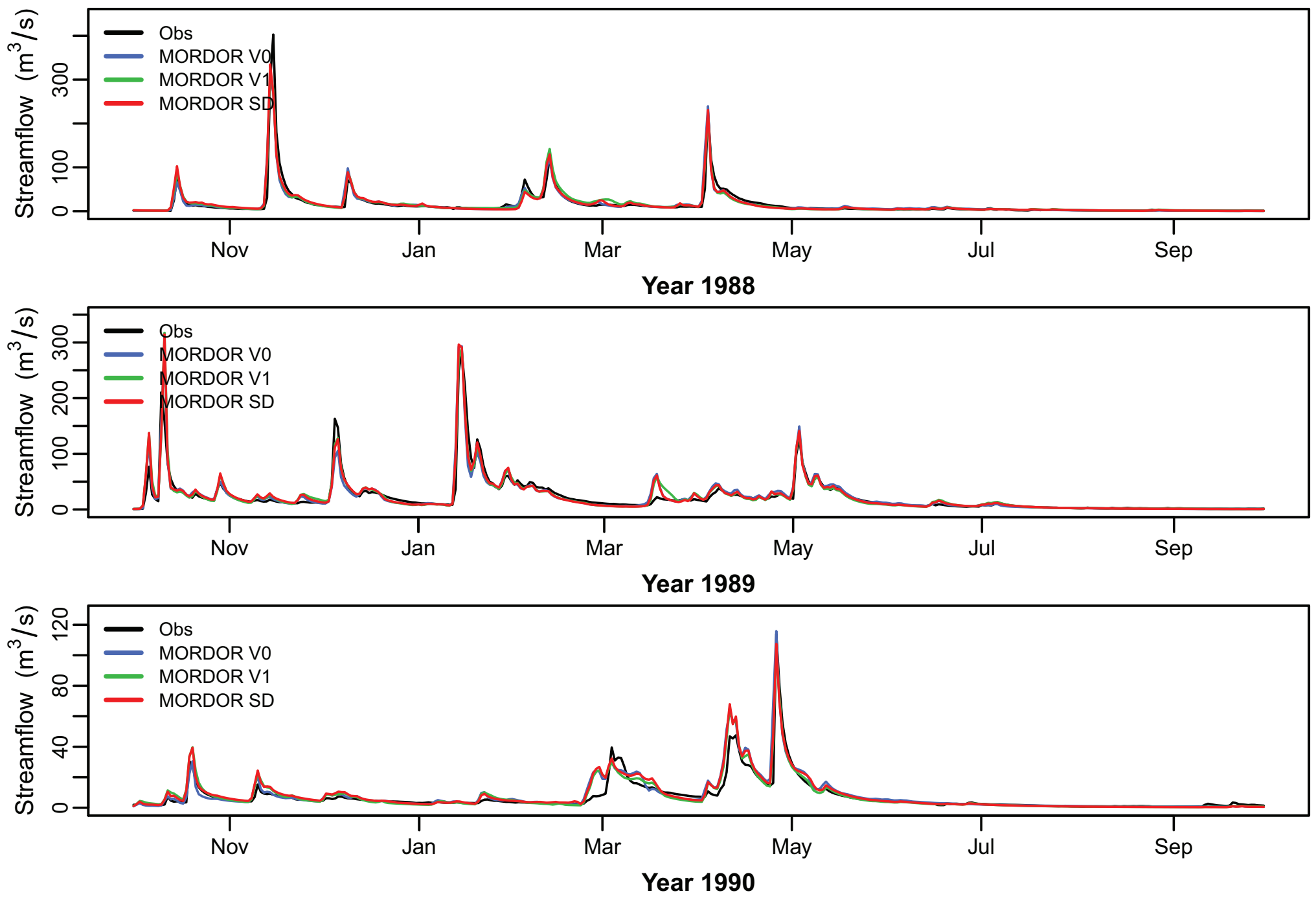

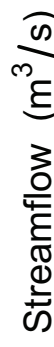




\section{Tarn@Montbrun}

\section{Year 1992}

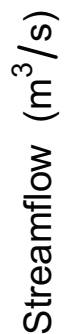

$=$ O

Obs

MORDOR V0

MORDOR V1

S

in-

Nov

Nov Jan
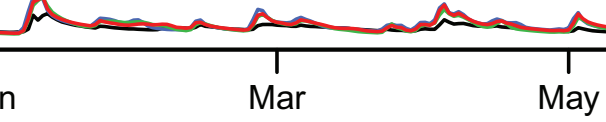

Year 1993

$\frac{0}{m}$
है
3
0
5
0
0
0

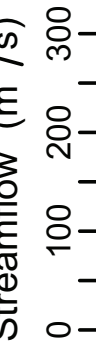

- Obs

- MORDOR VO

- MORDOR V1

MORDOR SD

-
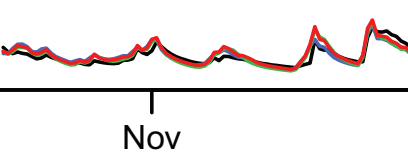

Jan

Mar

Year 1994

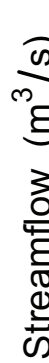
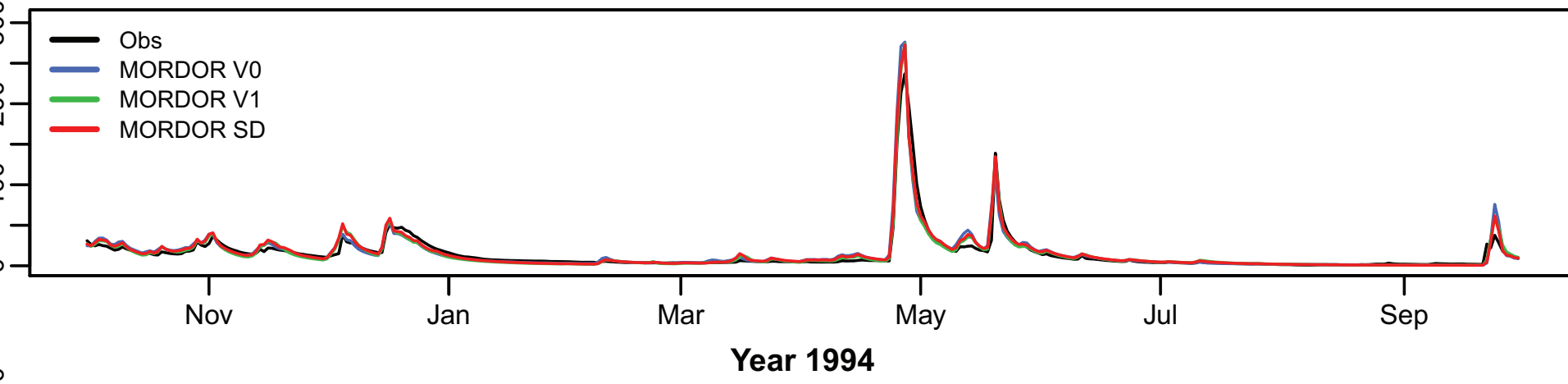

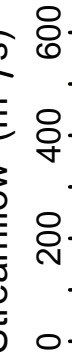

- Obs

- MORDOR V0

MORDOR V1

MORDOR SD

Nov $_{\text {Jan }}$

Year 1995

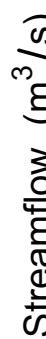

- Obs
- MORDO
MORDO
MORDO

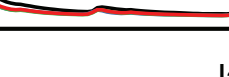

Jan
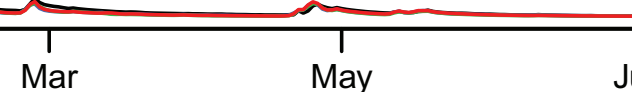

$\Lambda$

Year 1996
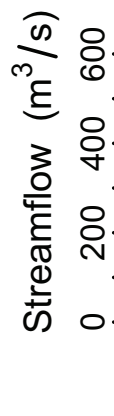

\section{ons}

$\longrightarrow$ MORDOR V0

$\longrightarrow$ MORDOR V1

MORDOR SD

mulanda

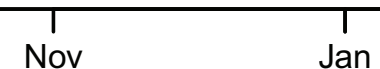

Jan

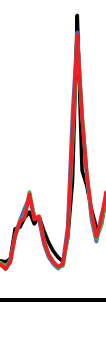

n
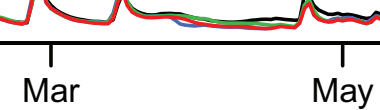

N

Year 1997
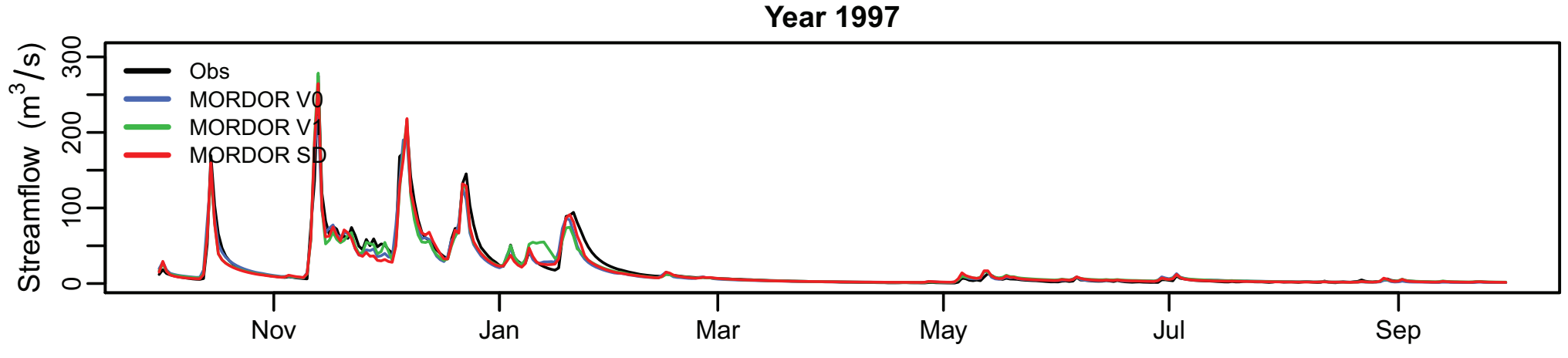


\section{Tarn@Montbrun}

Year 2004

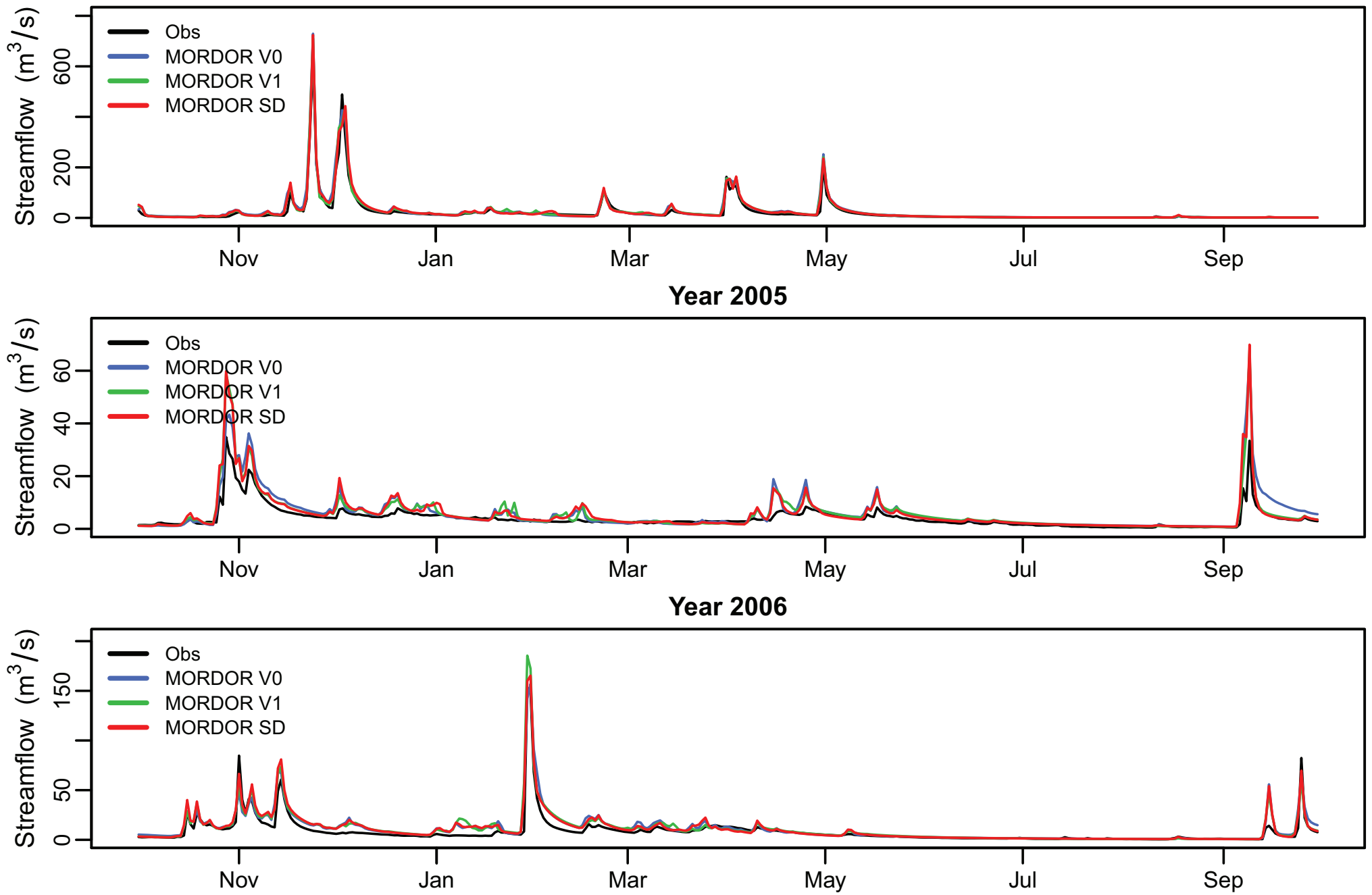


Tarn@Pinet

Year 1993

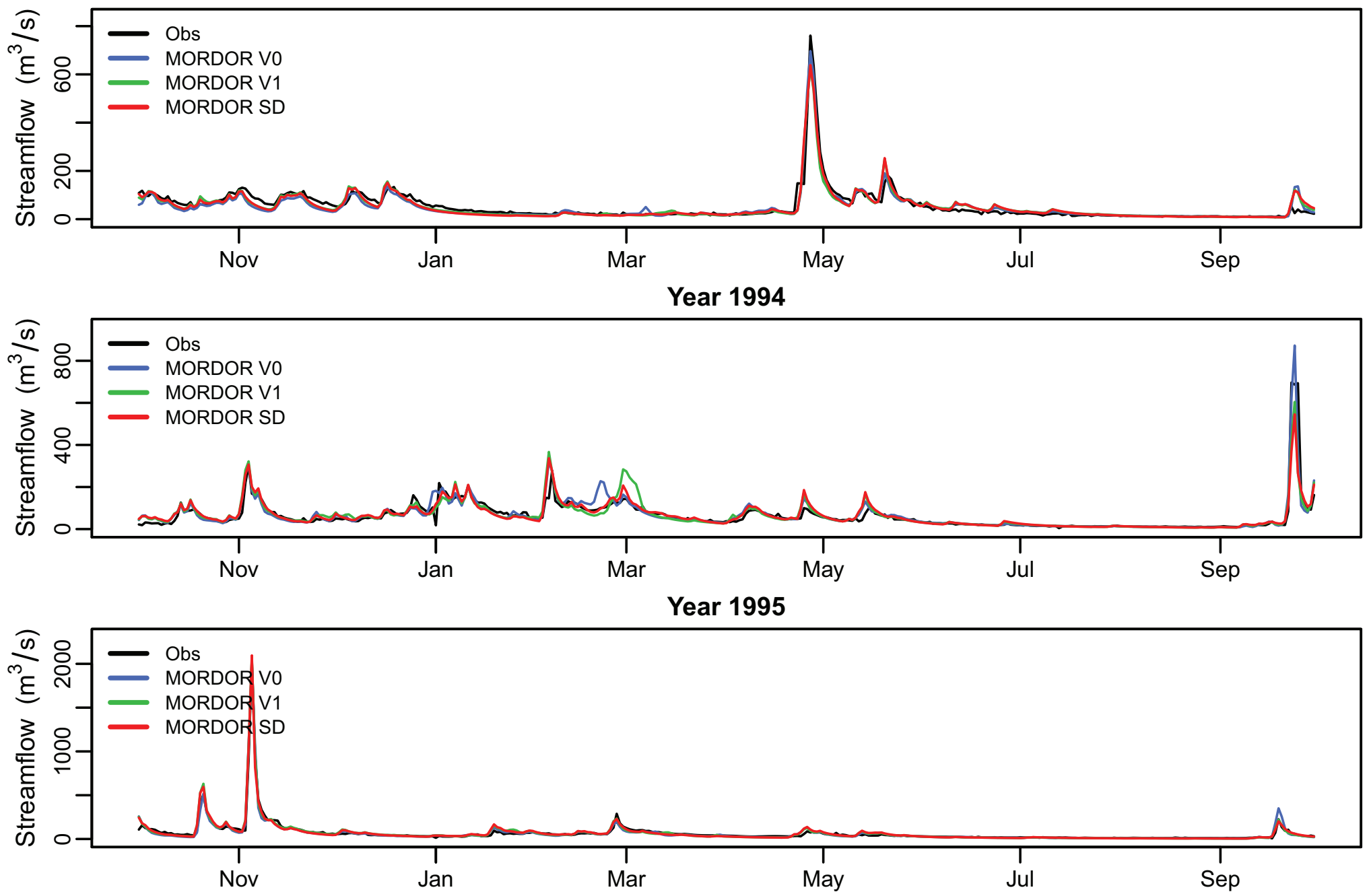


Tarnon@Florac

Year 2004
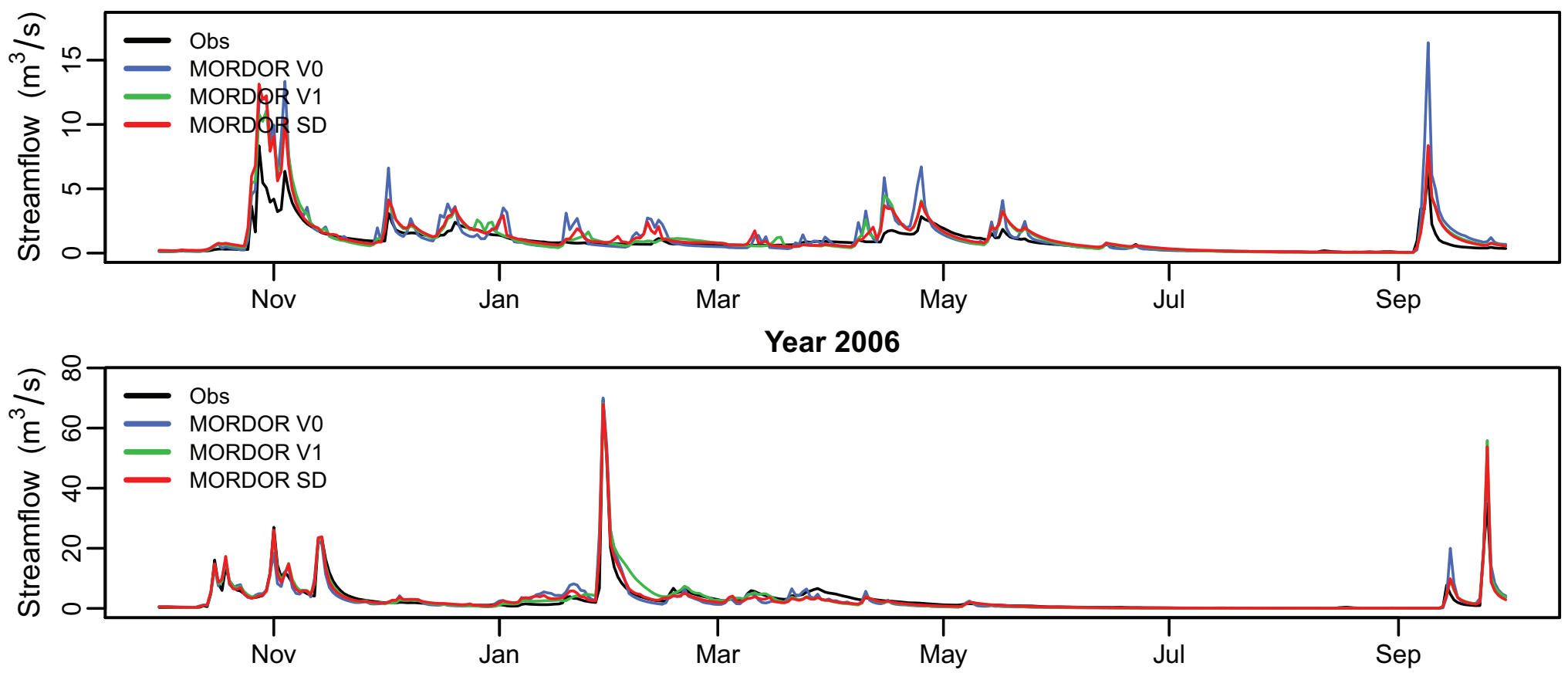
Taurion@RocheTalamie

Year 1964

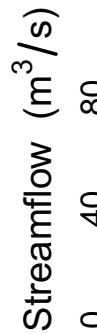

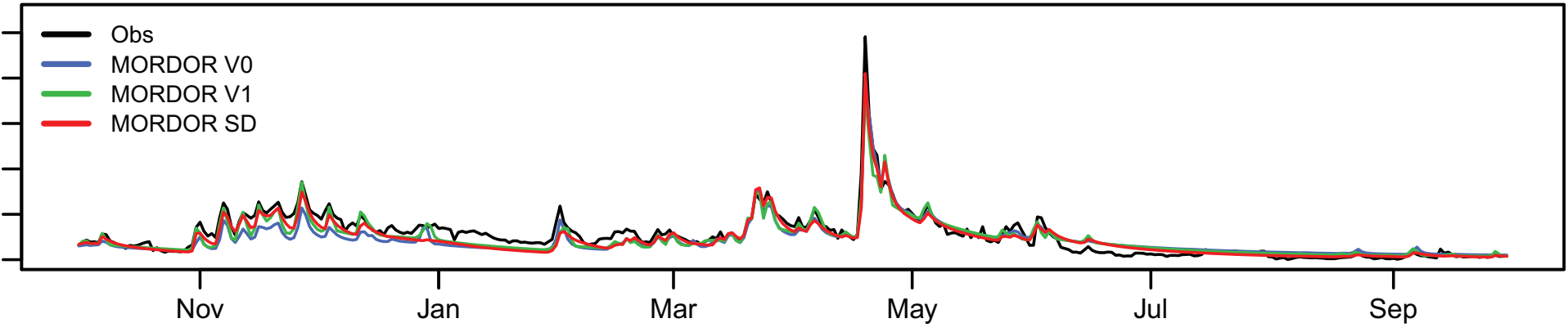

Year 1965

$\frac{\pi}{m}$
m
0
0
0

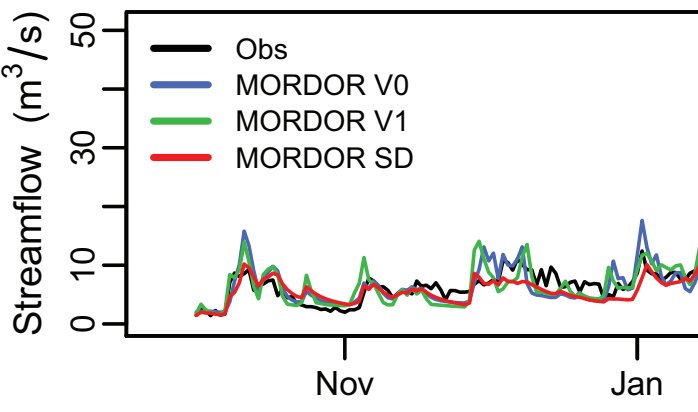

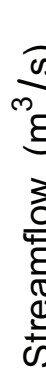

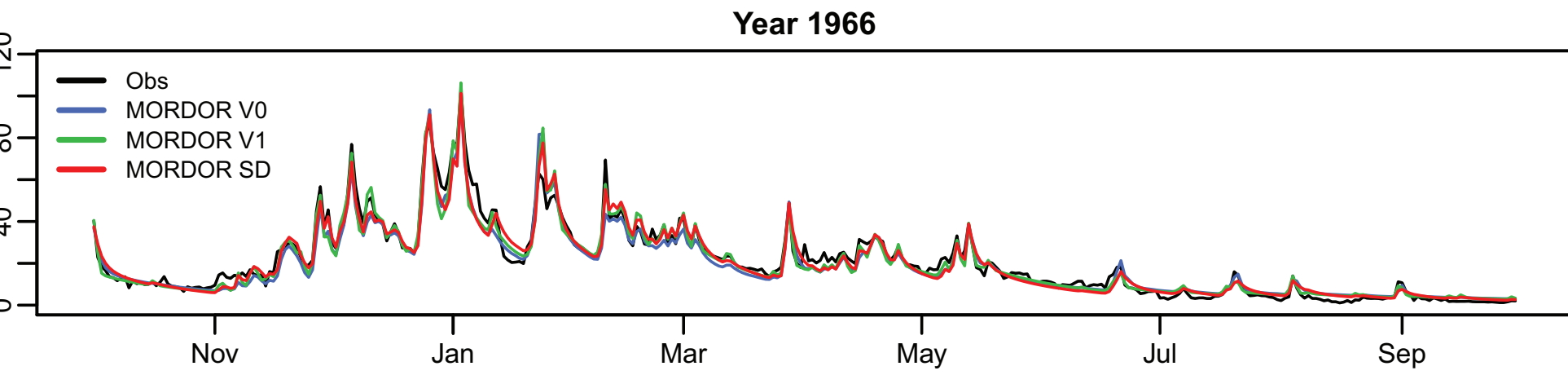

Year 1967

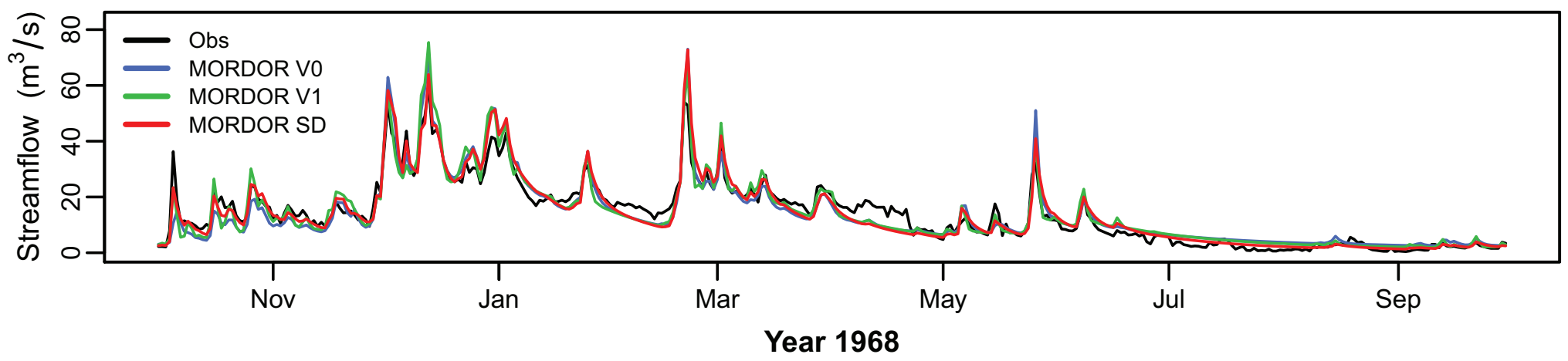

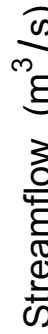
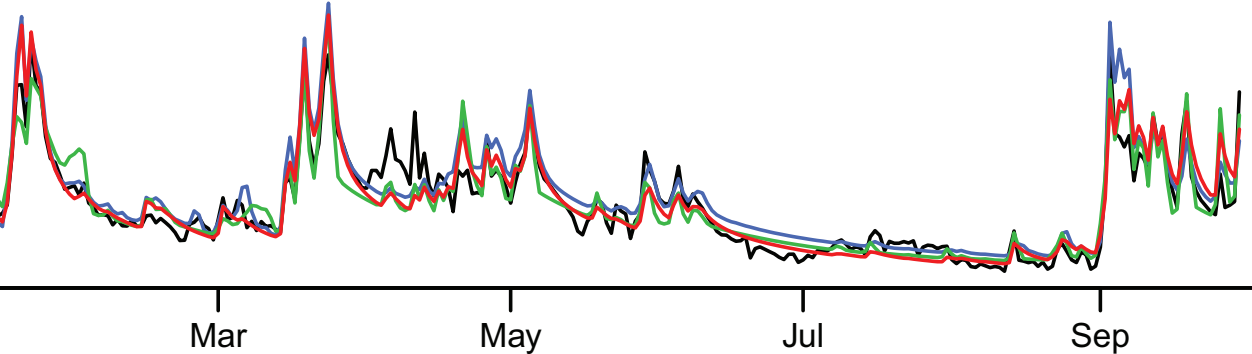

Amand Non

Sep 


\section{Taurion@RocheTalamie}

\section{Year 1970}
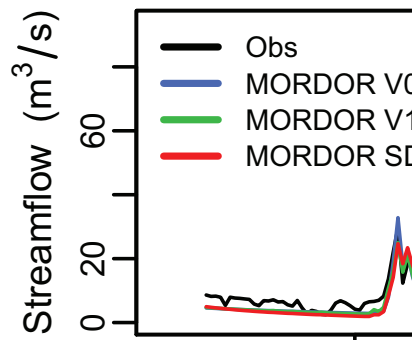

rm

Whath

Nov
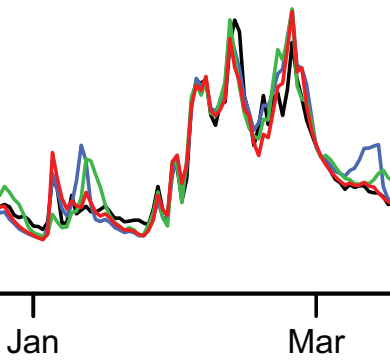

N
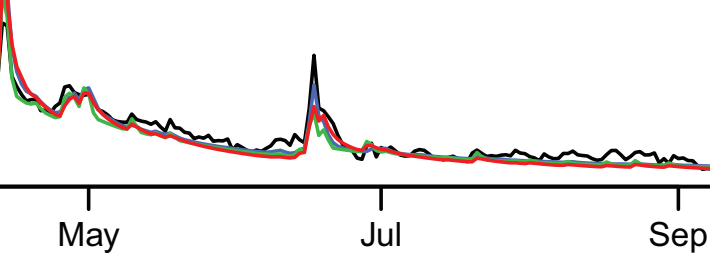

Year 1971
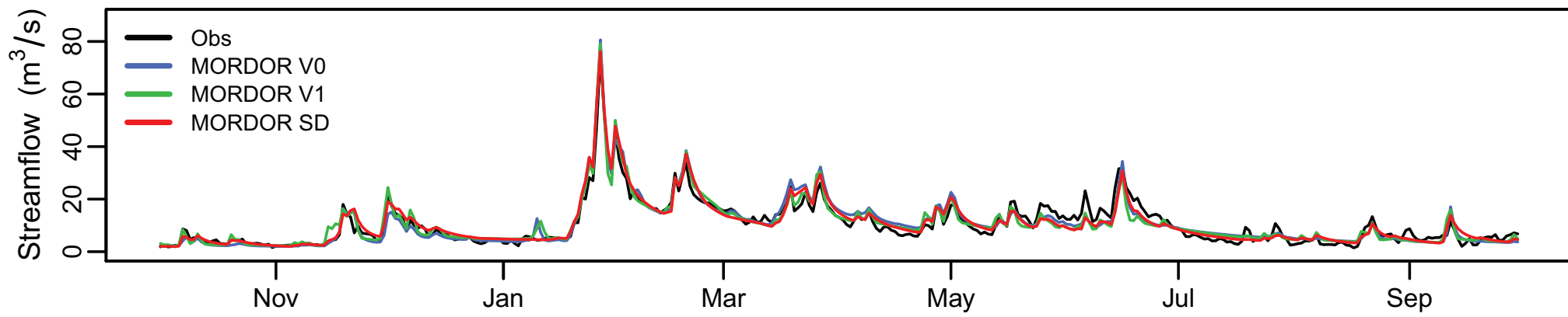

Year 1972

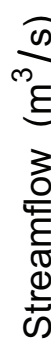

- Obs

- MORDOR V0

- MORDOR V1

- MORDOR SD

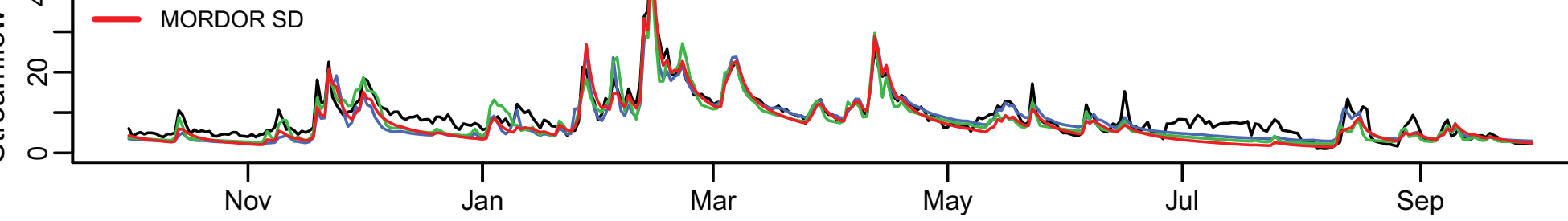

Year 1973
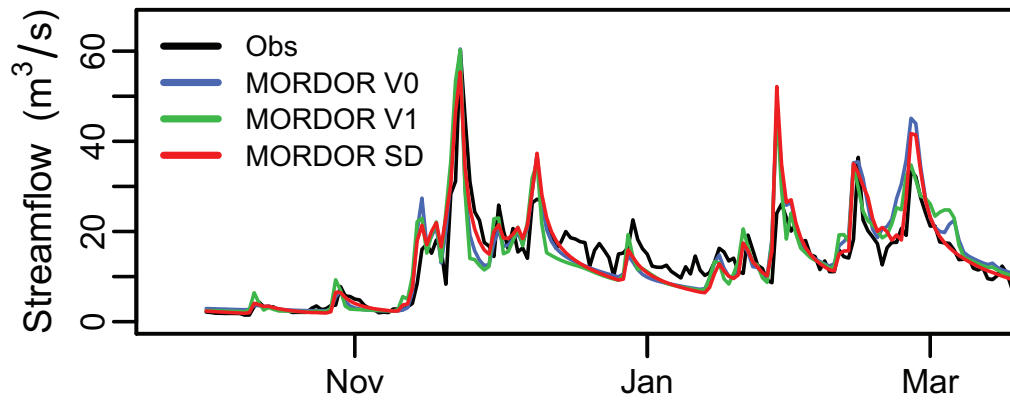

and

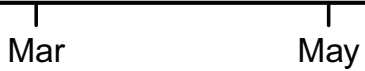

Year 1974
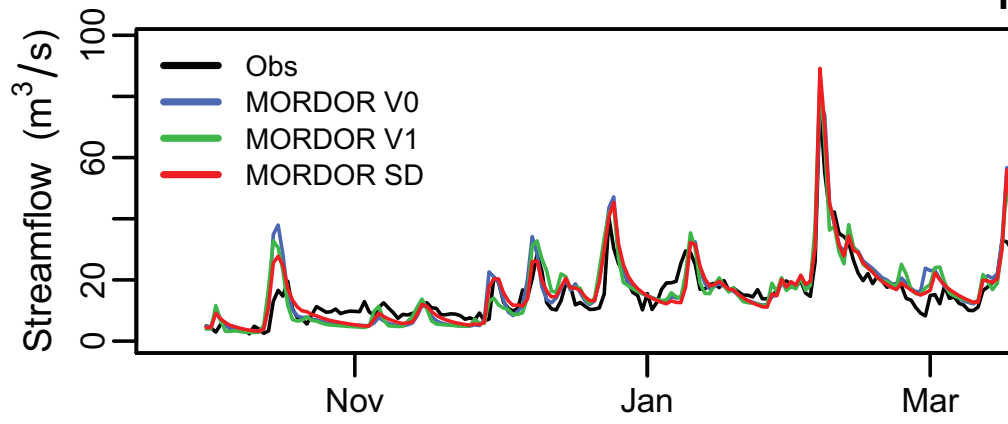

Year 1975
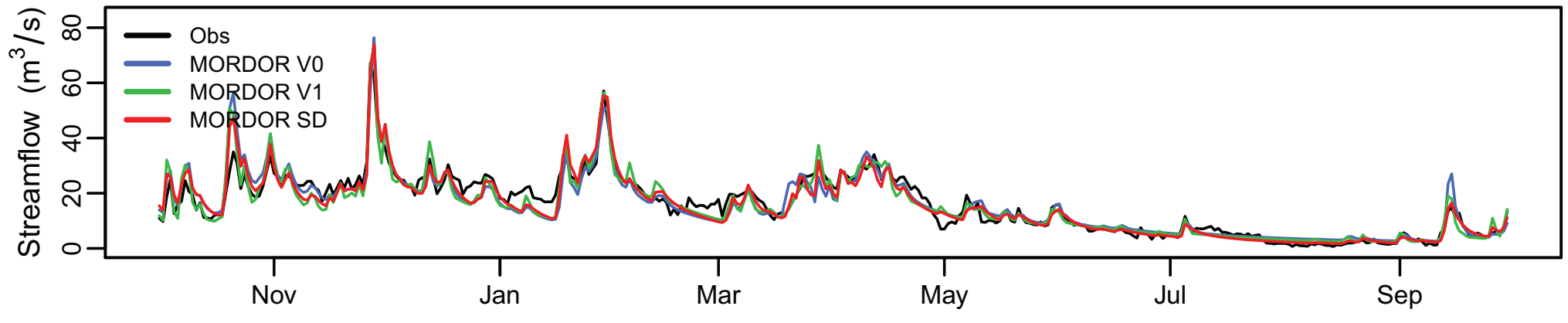


\section{Taurion@RocheTalamie}

\section{Year 1976}
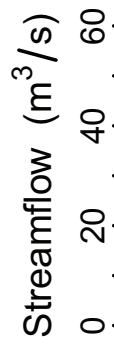

$\longrightarrow$ Obs
MORDOR V0
MORDOR V1
MORDOR S

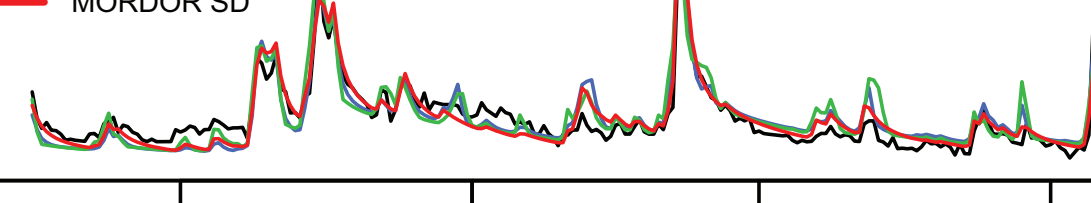

Nov

Jan

Mar

May

Year 1977

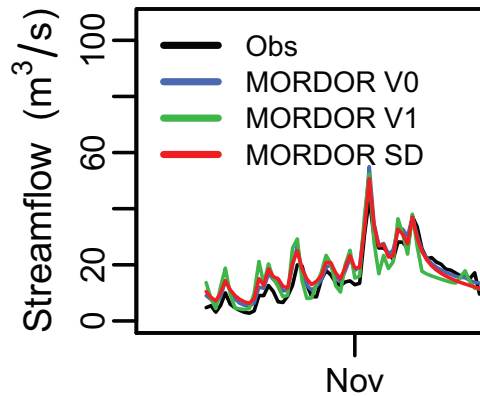
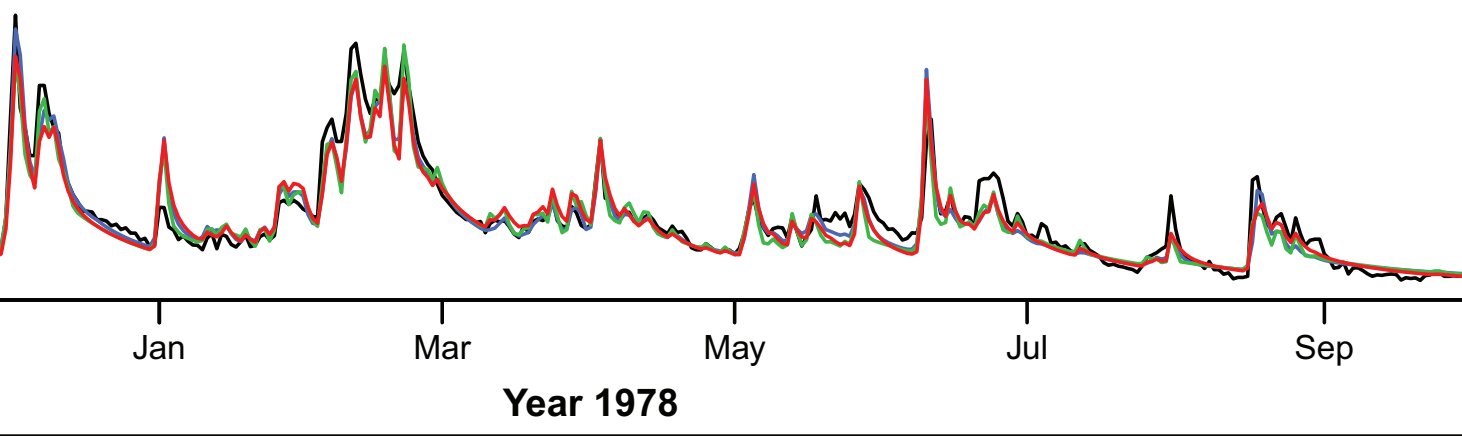

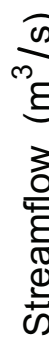

- Obs

- MORDOR V0

- MORDOR V1

- MORDOR SD

垔

Nov $\operatorname{Jan}_{\text {Mar }}^{1}$ May $\quad$ Jul

Year 1979

0
0
0
0
0

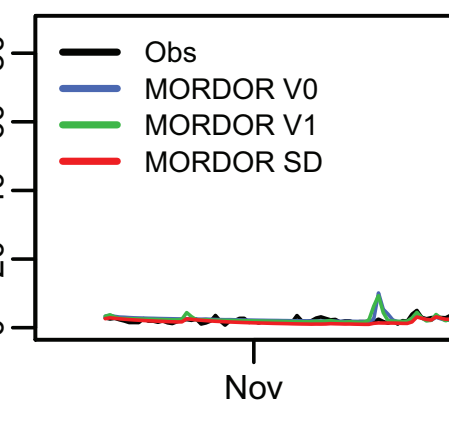

As
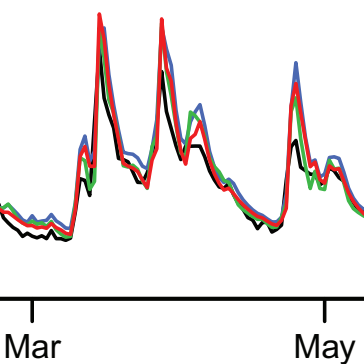

Year 1980
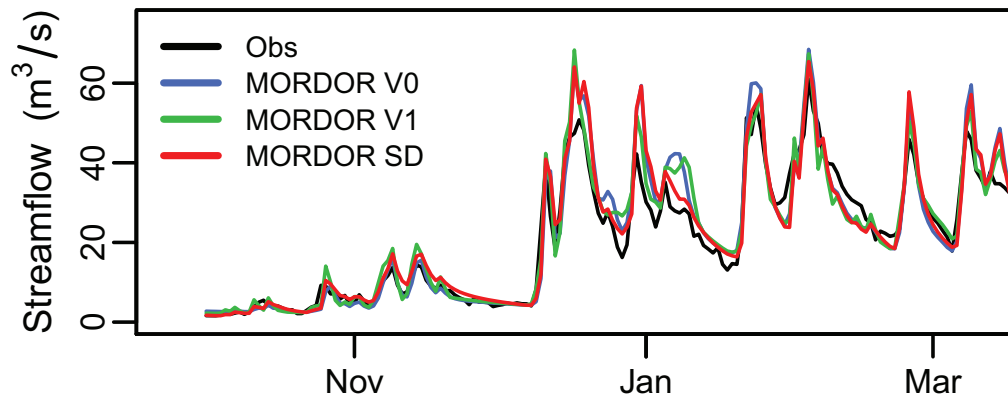

Year 1981
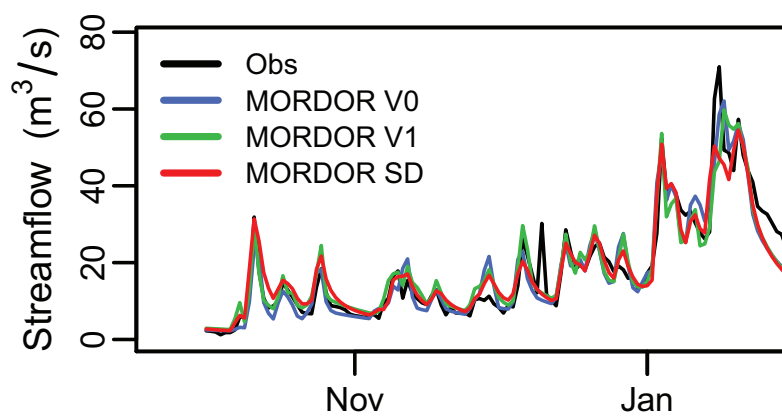


\section{Taurion@RocheTalamie}

\section{Year 1988}

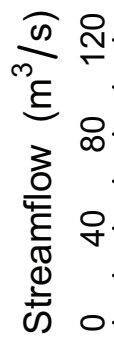

- Obs
MOR
MOR
MOR
A

A M

Mama $\mathbb{N}^{N}(\operatorname{man}$
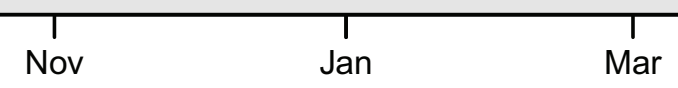

Year 1989

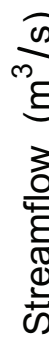

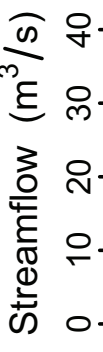

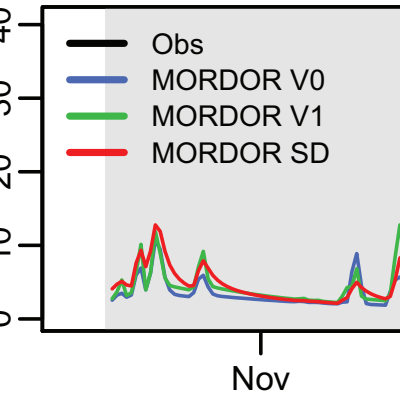

and

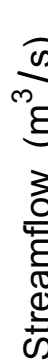

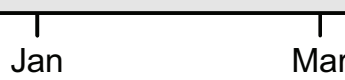

Year 1990

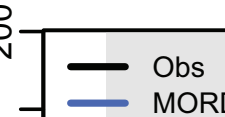

- $=$ MORDOR V0

으 MORDOR SD

으-

o-

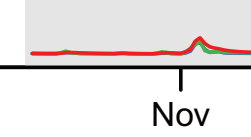

$\overbrace{\text { Nov }}^{\text {r }}$

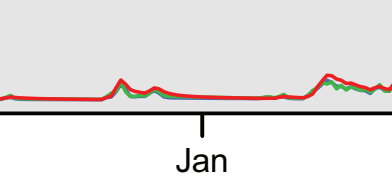

Jan

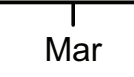

Year 1991
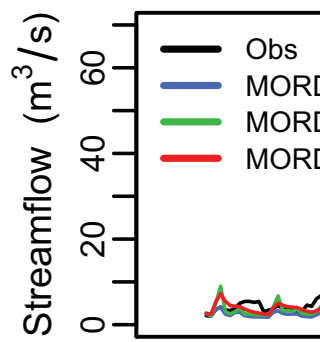

\begin{tabular}{l}
-10 \\
$O R$ V 0 \\
\hline
\end{tabular}

PQR SD

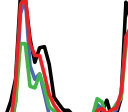

$M A c^{2}$
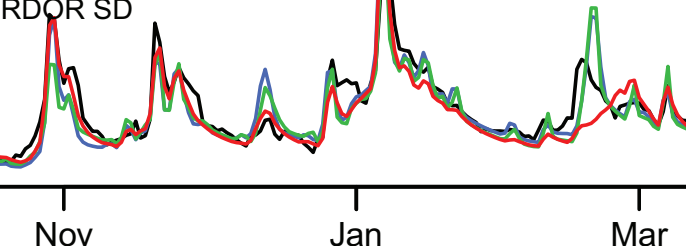

Jan

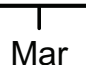

andaras May

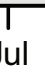

Sep 
Taurion@RocheTalamie

Year 2000

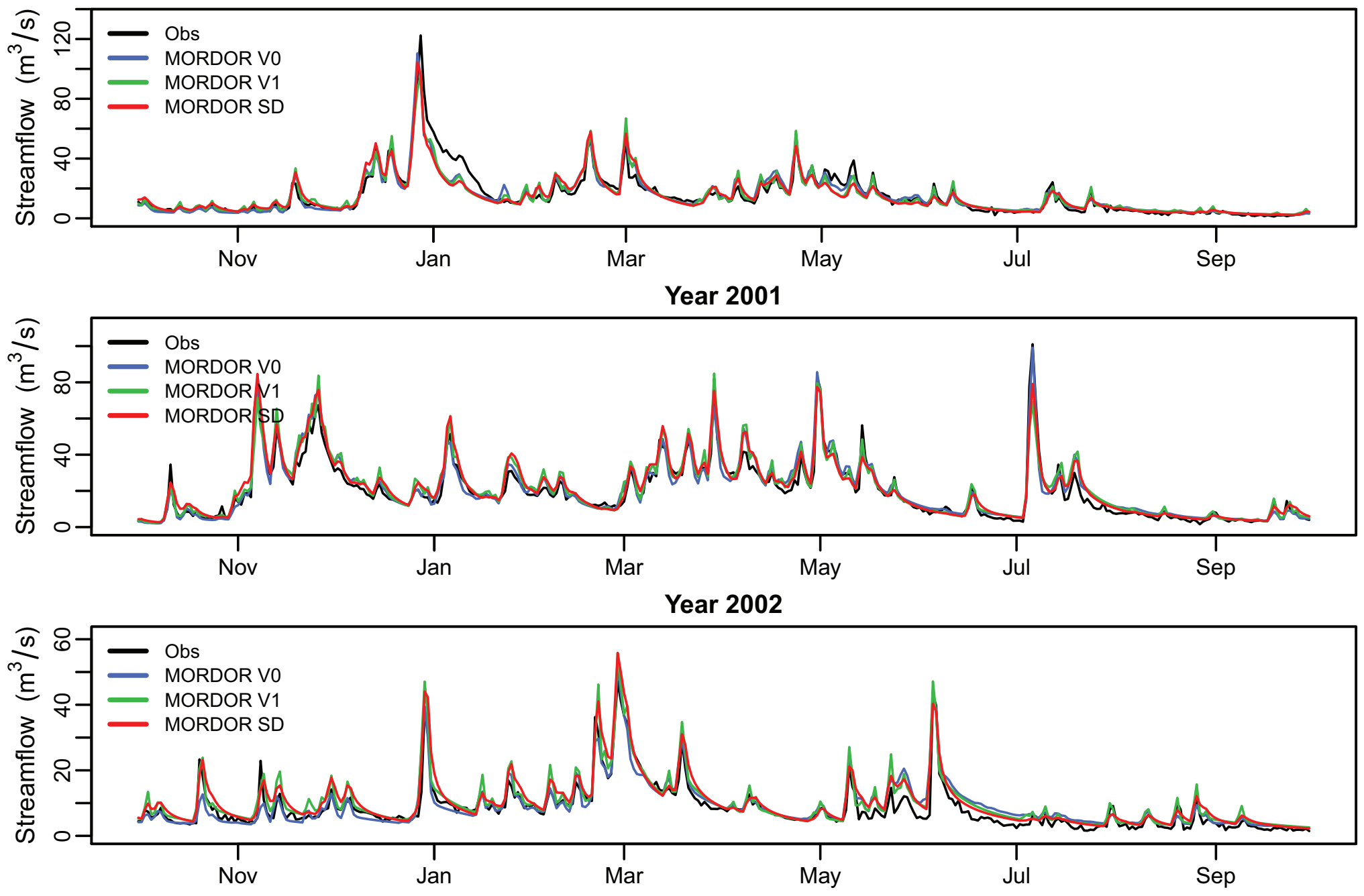




\section{Tet@Vinca}

Year 1989
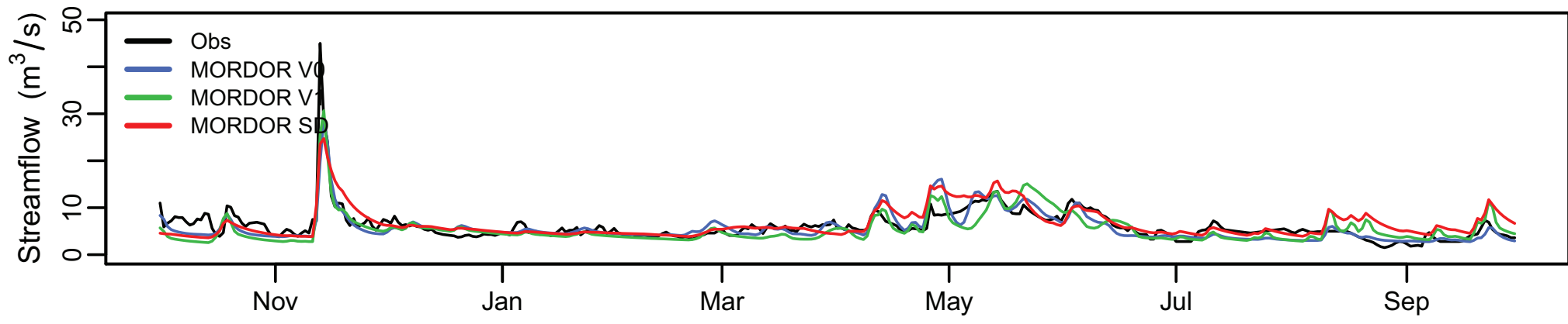

Year 1990
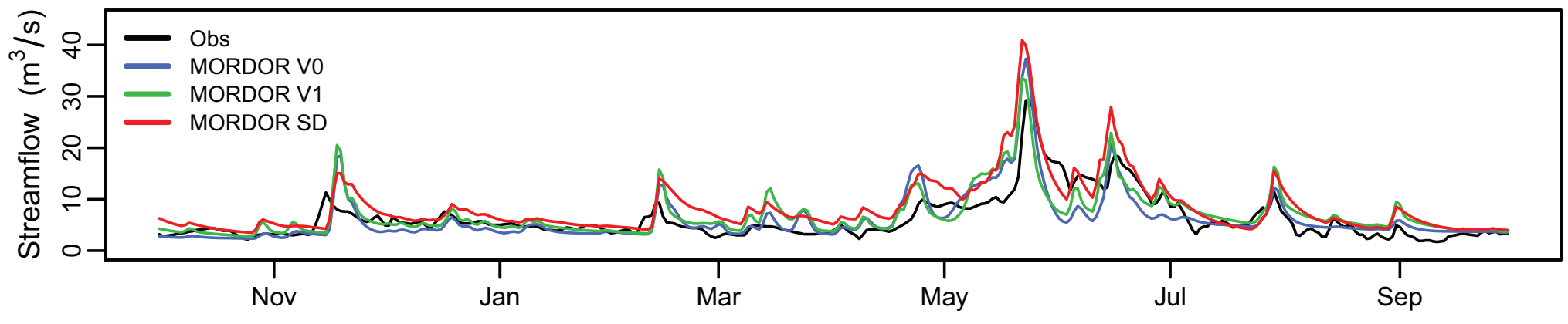

Year 1991

$\frac{0}{m}$
$m^{2}$

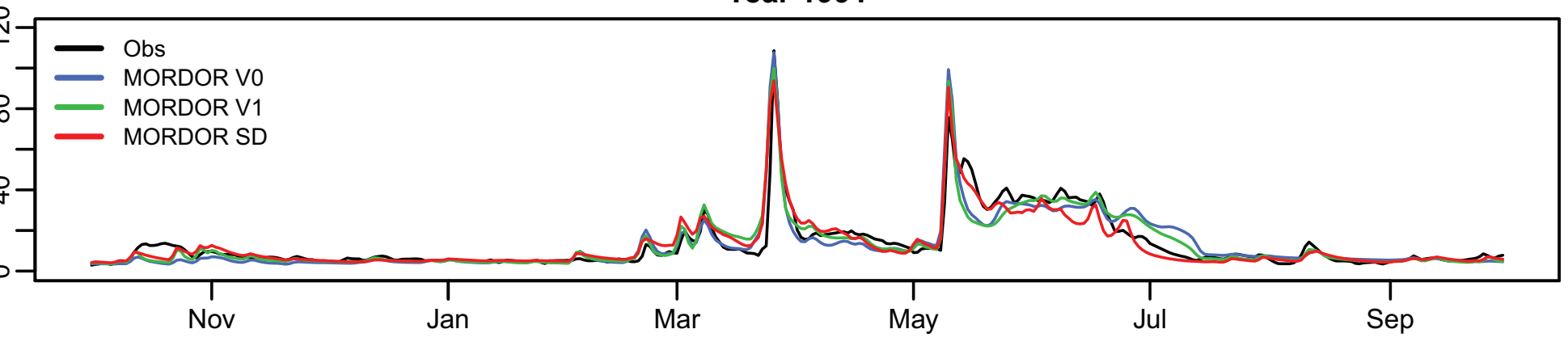

Year 1992
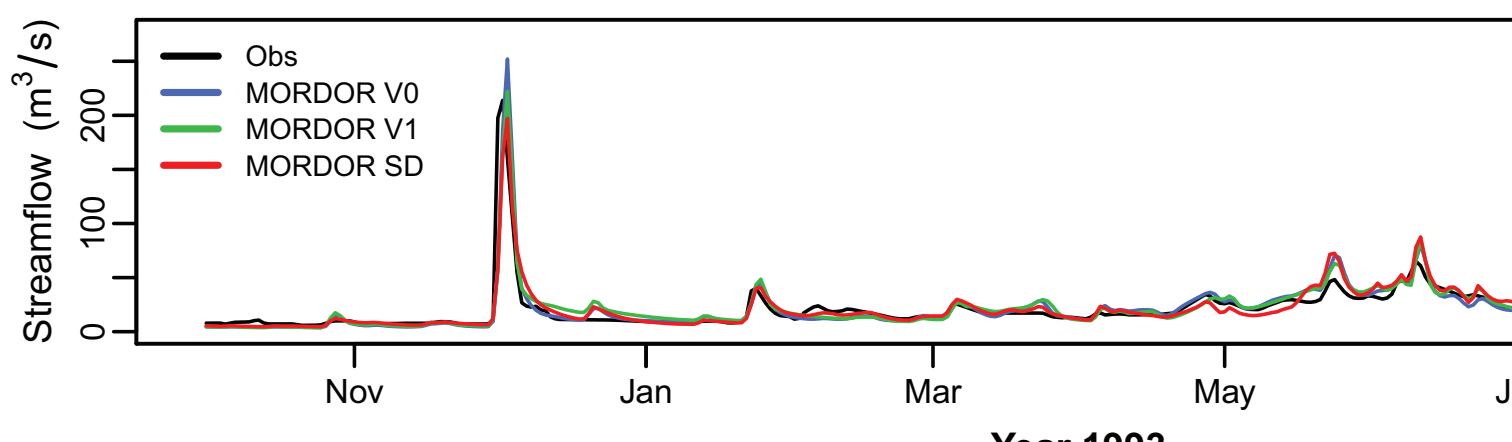

Year 1993
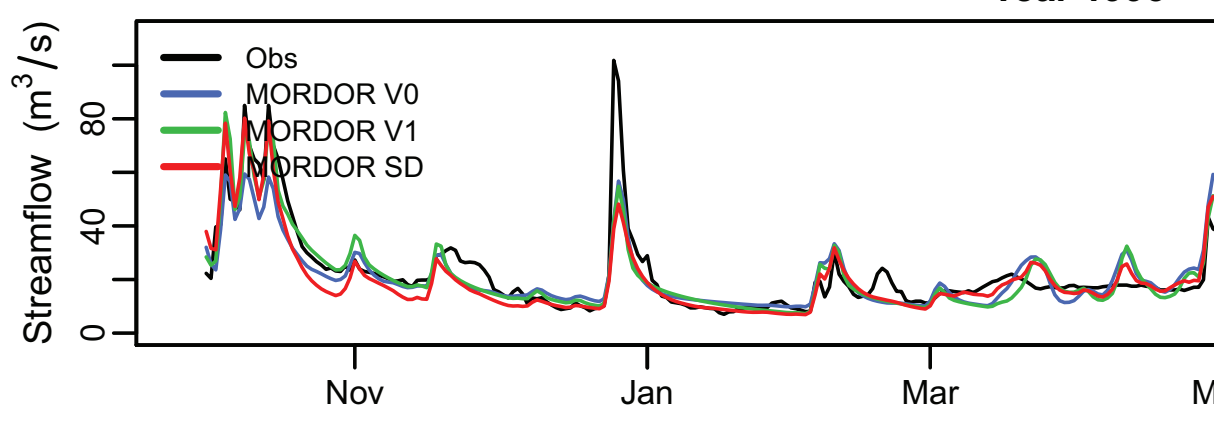

Year 1994
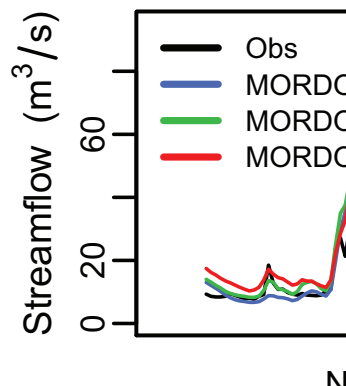


\section{Tet@Vinca}

Year 2001
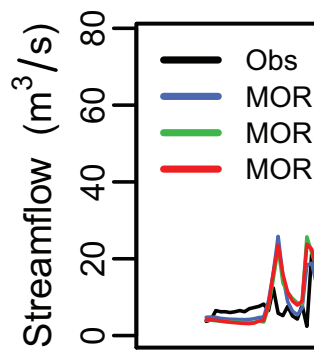

MORDOR V0

MORDOR V1

- MORDOR SD

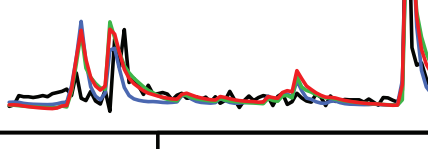
Nov

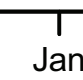

Naras

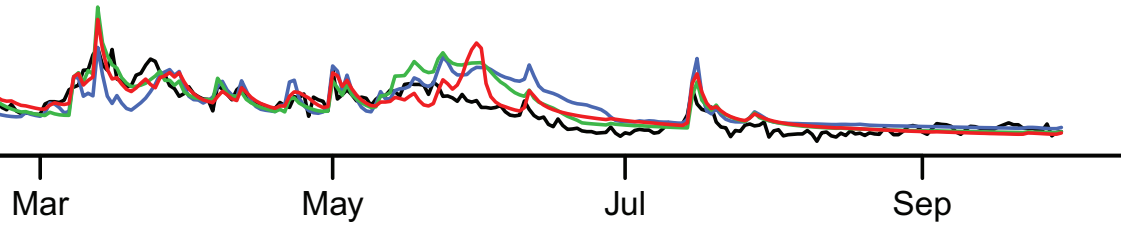

Year 2002
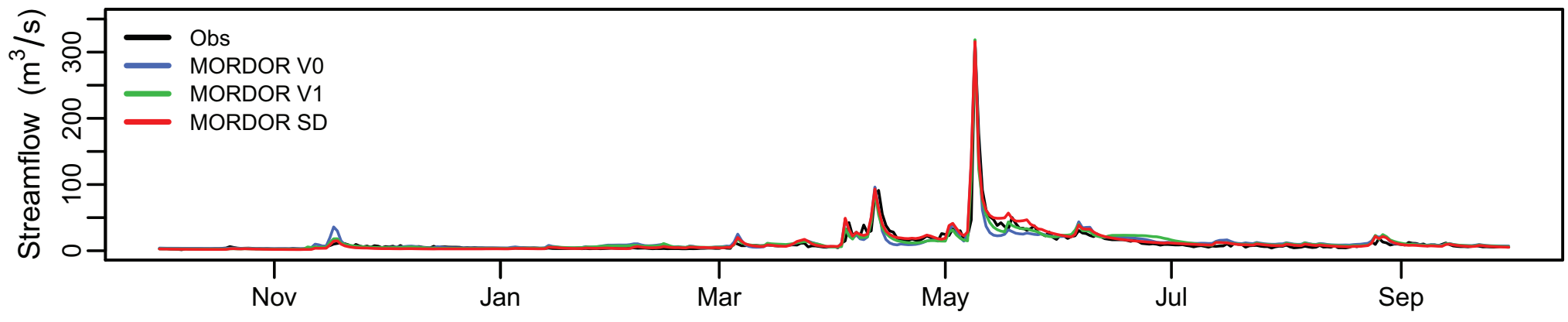

Year 2003

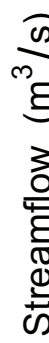

- Obs

- MORDOR V1

MORDOR SD

$\operatorname{Nov}_{\text {Nov }}^{\text {Jan }}$

Year 2004

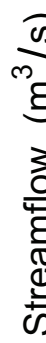

- Obs

- MORDOR V0

MdRDOR SD

岳

in-

o-

MQRDOR SD

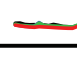

Nov

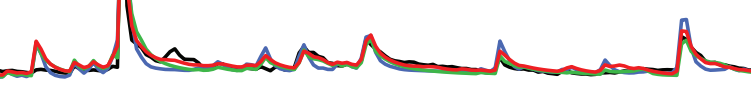

Jan Mar

Year 2005

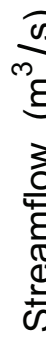

Obs

- MORDOR VO

- MORDOR V1

ㄱ-

o-

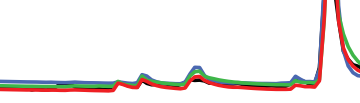

Nov

andan

Jan

Mar

Year 2006
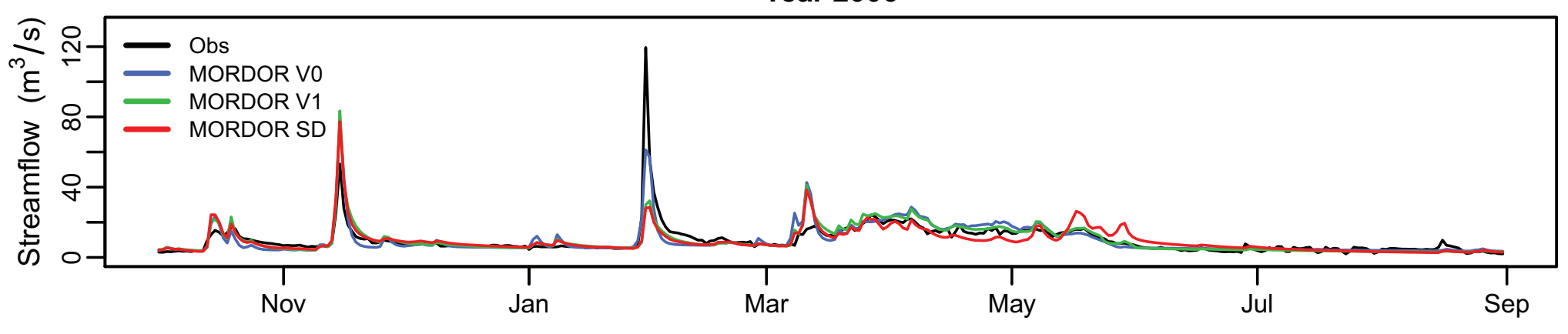


\section{Truyere@Grandval}

Year 1980

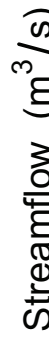

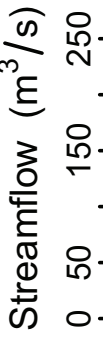

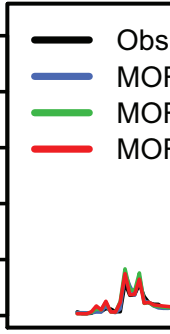

Obs
MORDOR V0

MORDOR V1

ORLOR SD

Nov

Nov

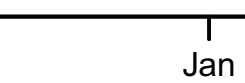

Jan Mar

Year 1981
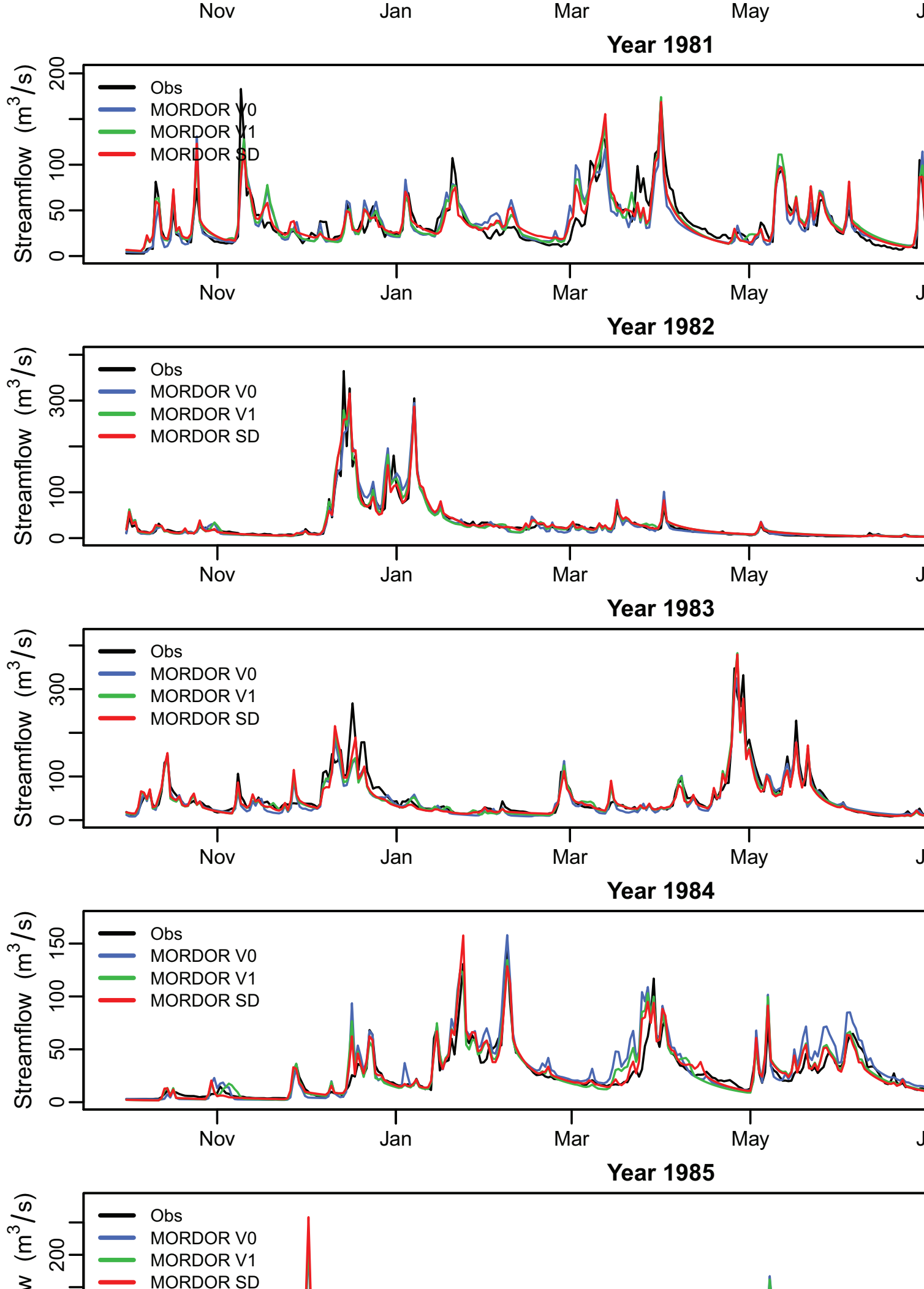

Year 1982
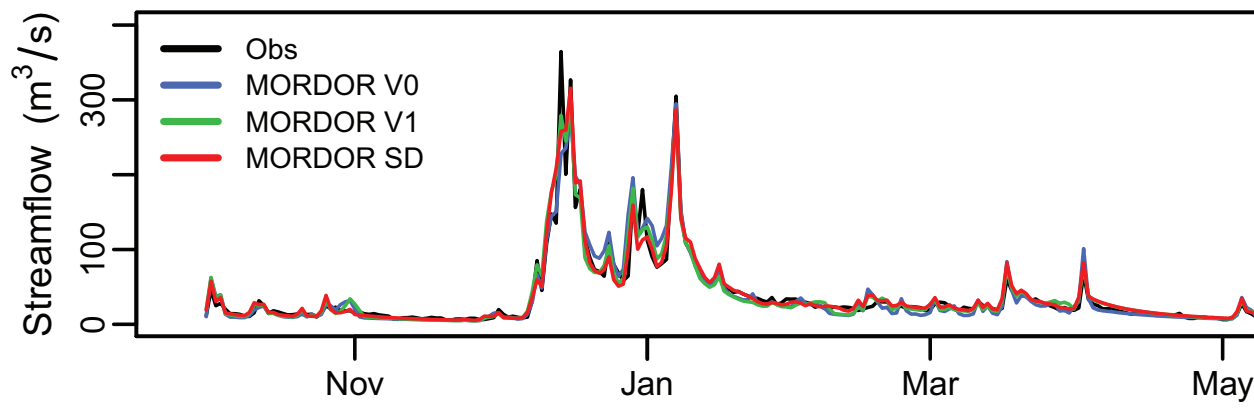

Year 1983
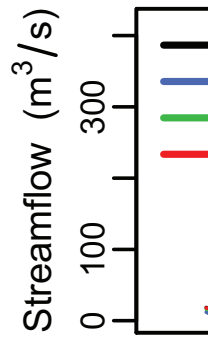

\section{Obs}

MORDOR V0

MORDOR V1

MORDOR SD

Man Amand

Nov
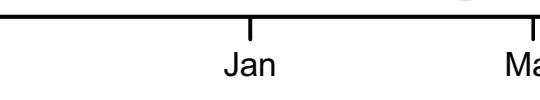

Mar

Year 1984

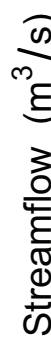

कृ

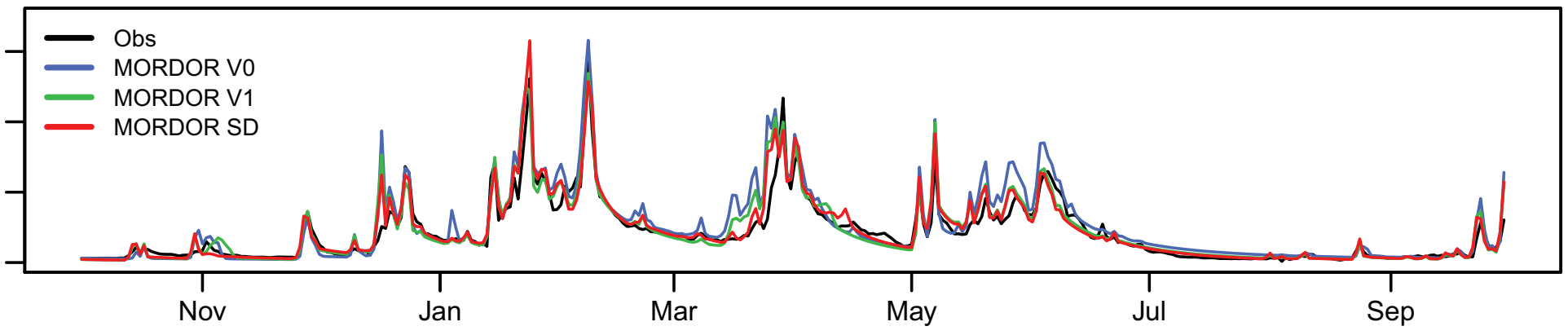

Year 1985
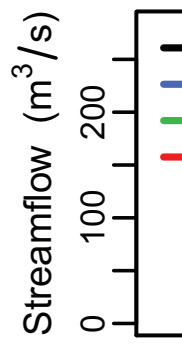

Obs

- MORDOR V0

MORDOR V1

MORDOR SD

du

huned

Sep

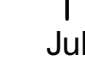




\section{Truyere@Grandval}

Year 1986
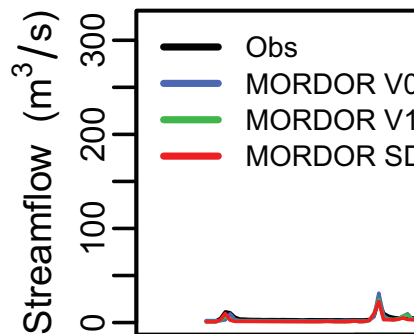

$\longrightarrow$ MORDOR VO

$\longrightarrow$ MORDOR V1

MORDOR SD

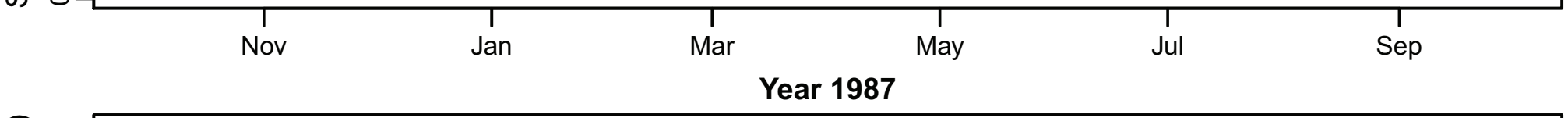

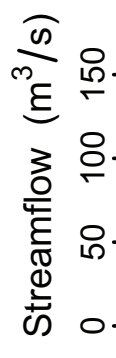

$\longrightarrow$ Obs

- MORDOR V0

$\longrightarrow$ MORDOR V1

- MORDOR SD

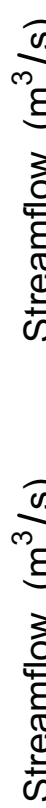

- Obs

只- MORDOR V1

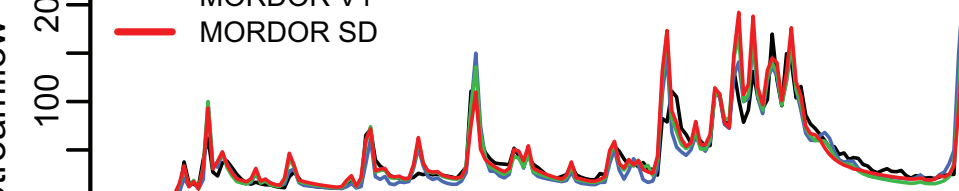

Nov Jan $_{\text {Mar }}^{1}$

Year 1989

0
$\frac{0}{m}$
5
3
0
0
0
0
0
0

只

- Obs

MORDOR V1

$\longrightarrow$ MORDOR SD

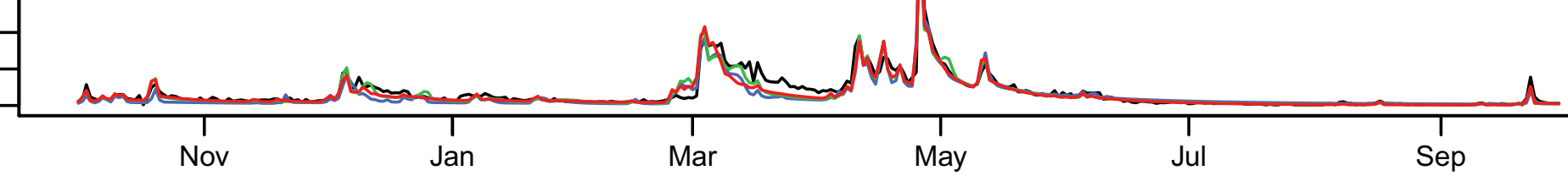

Year 1990

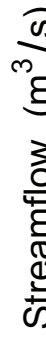

$\longrightarrow$ Obs

- MORDOR VO

MORDOR V1

- MORDOR SD

$\rightarrow$

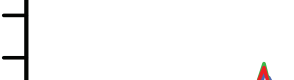

$-$

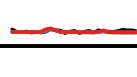

Nov

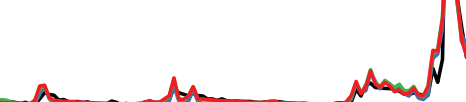

an
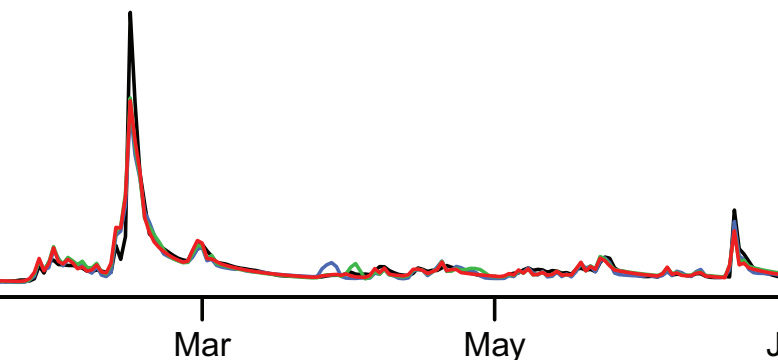

Year 1991
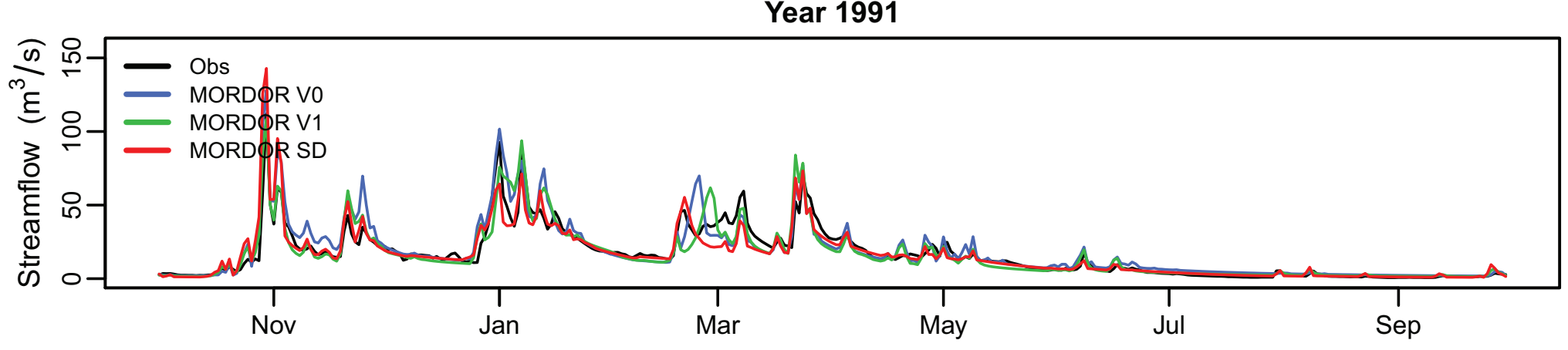
Truyere@Grandval

Year 2004

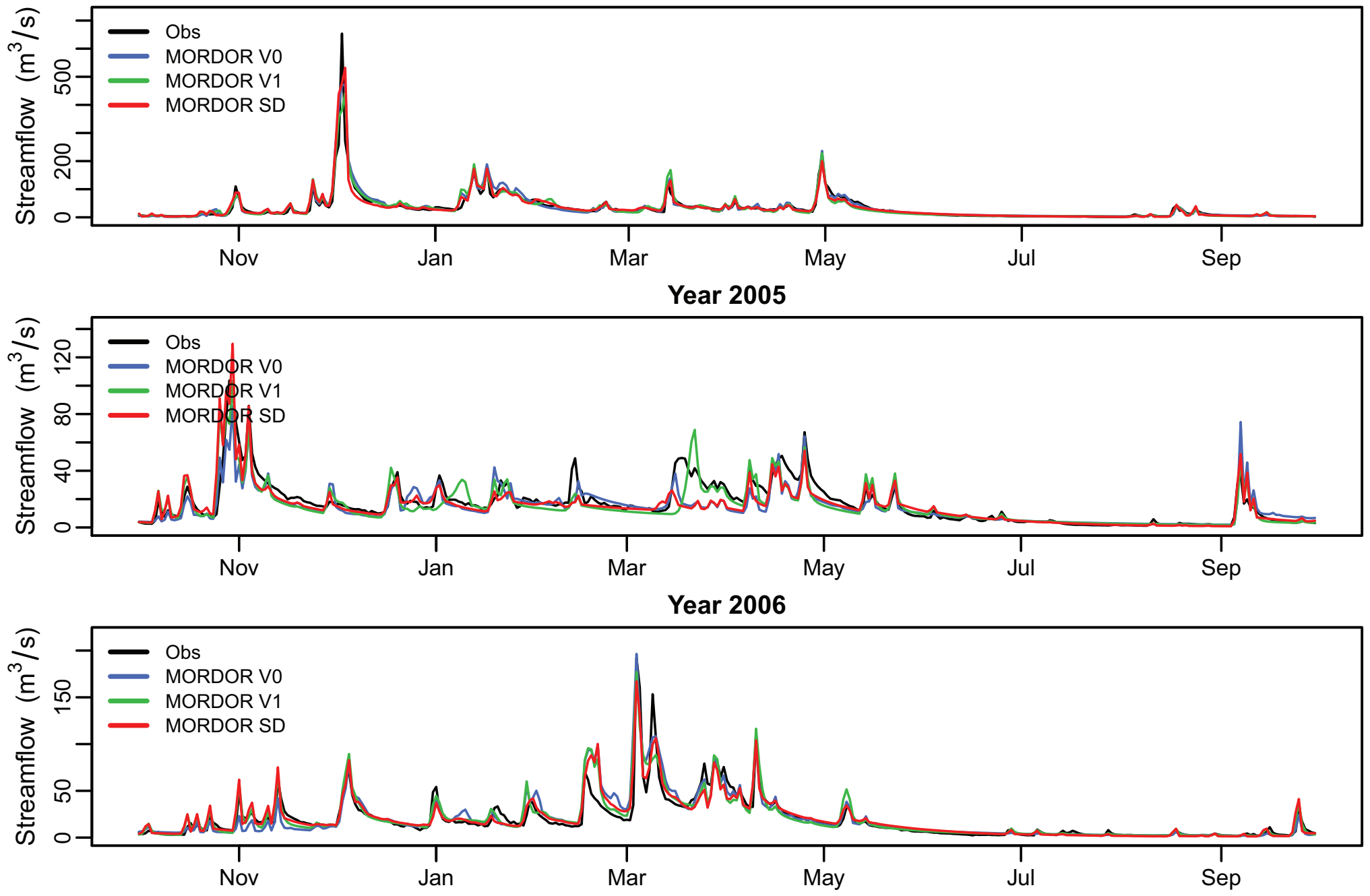




\section{Ubaye@RocheRousse}

Year 1983
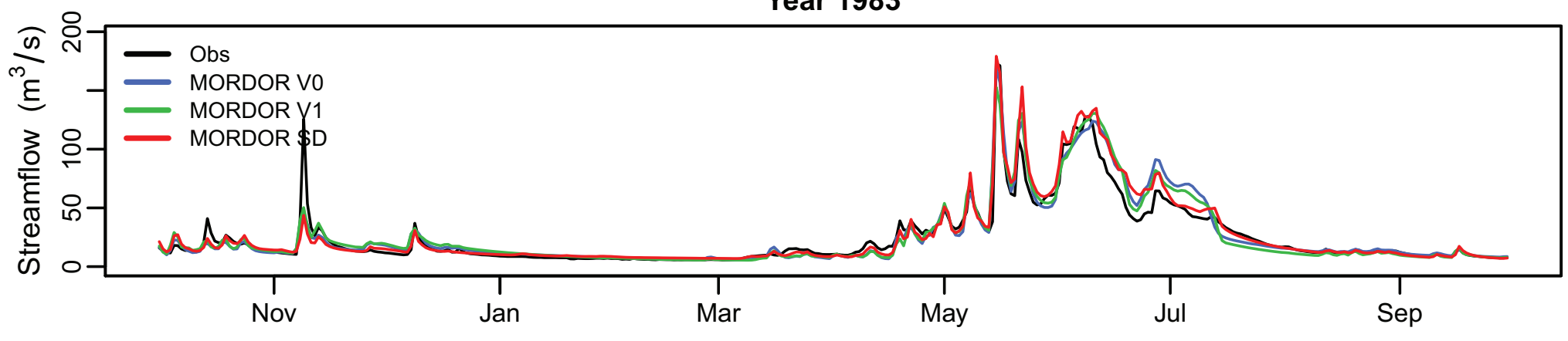

Year 1984
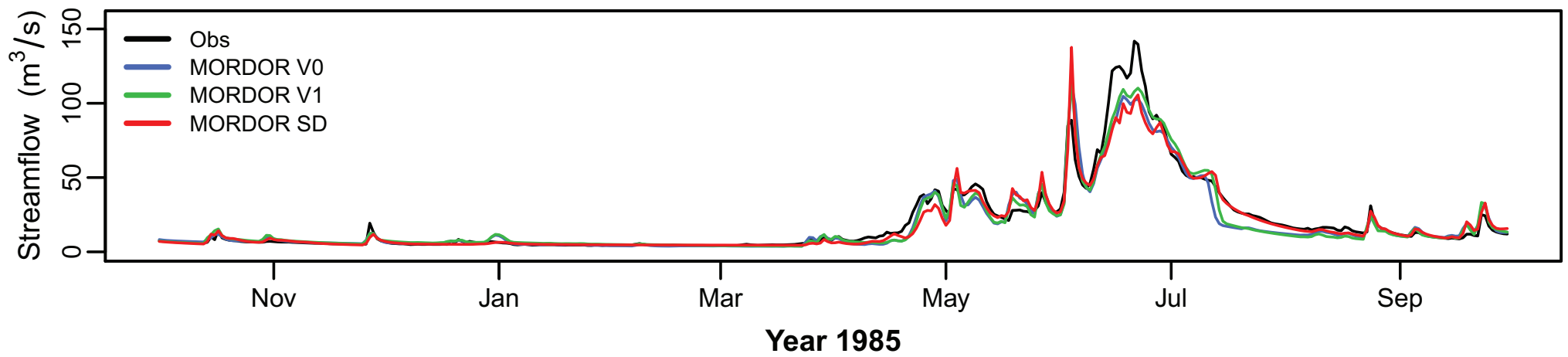

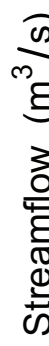

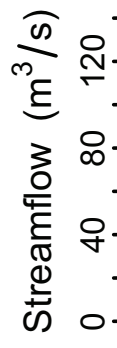

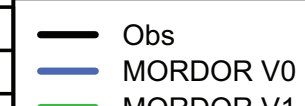

- MORDOR V1

- MORDOR SD

Man
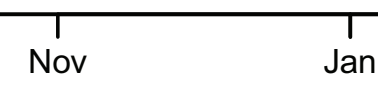

an

Mar

Mar May

Year 1986

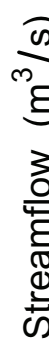

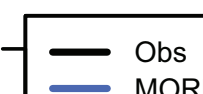

- $-\begin{aligned} & \text { MORDOR V0 } \\ & \text { MORDOR V1 } \\ & \text { MORDOR SD }\end{aligned}$

iᄋ-
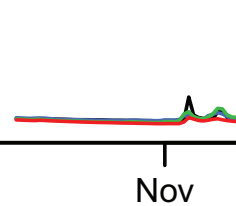

Nov

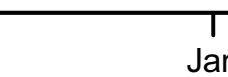

Jan

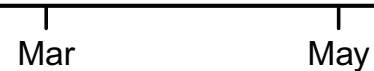

Year 1987
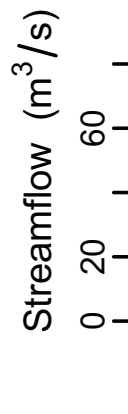

$\longrightarrow$ Obs

- MORDOR VO

- MORDOR V1

- MORDOR SD
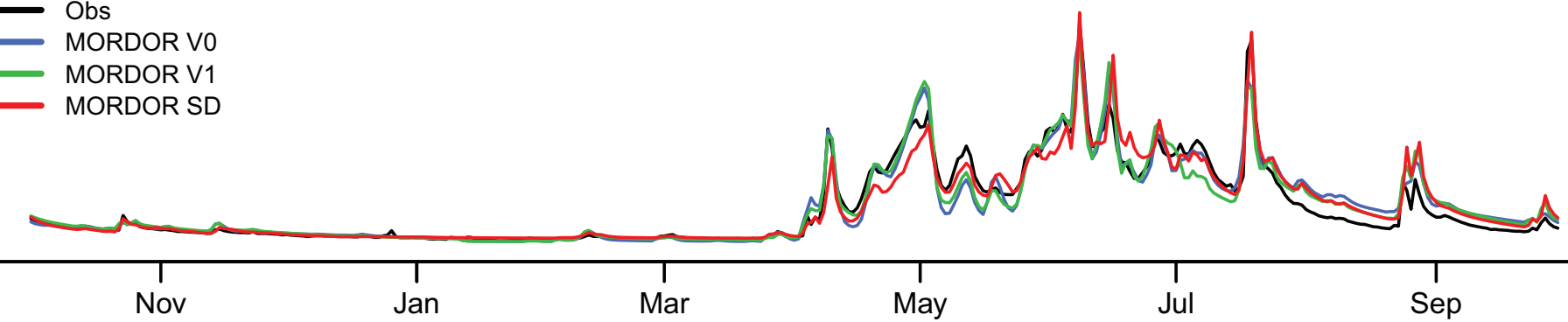

Jul

Sep

Year 1988

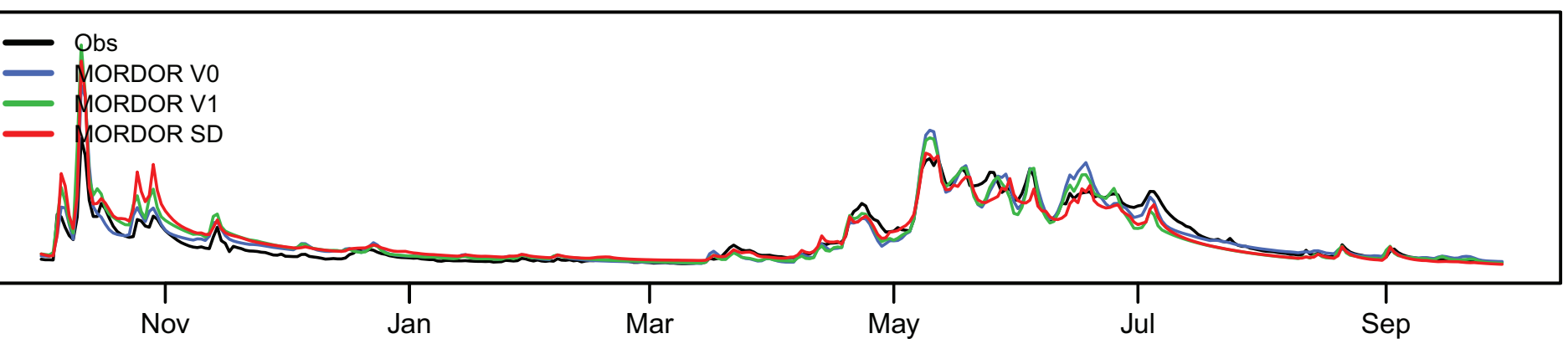


Vienne@Bussy

Year 1996

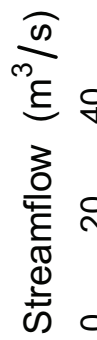

- Obs

- MORDOR V0
- MORDOR V1

- MORDOR SD

lundunt

Nov

Jan
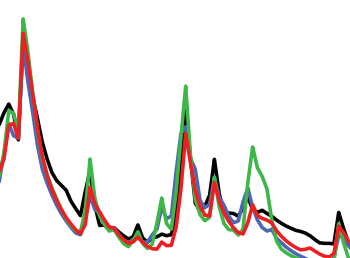

thand

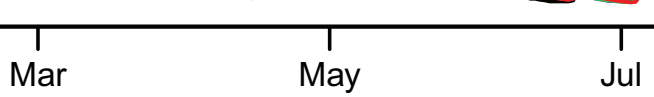

Year 1997

क

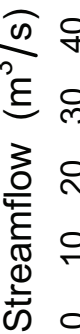

- Obs

- MORDOR V0

MORDOR V1

- MoRoorso

-

Nov

Nov
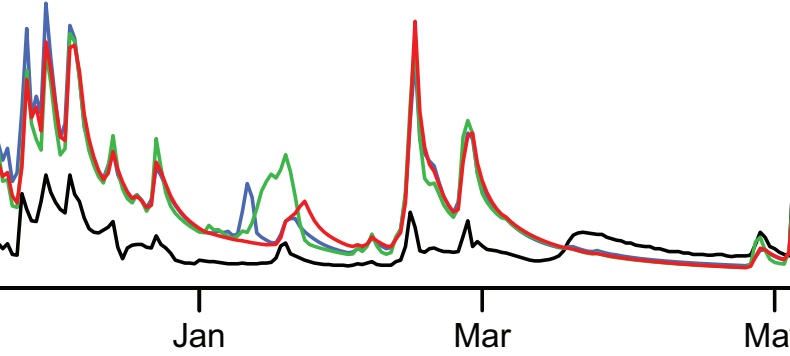

sin
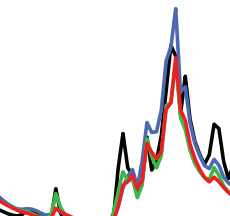

ha

Jan Mar

Year 1998

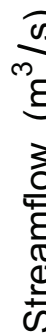

$-\begin{aligned} & \text { Obs } \\ & \text { MORDOR VO } \\ & \text { MORDOR V1 }\end{aligned}$

- MORDOR SD

an A

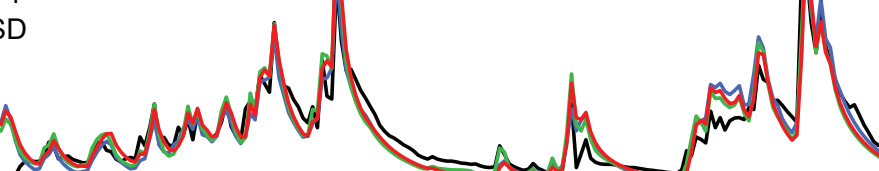

Nov

Jan

Mar May

Year 1999

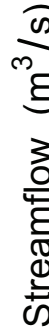

要

OR V0

DOR SD

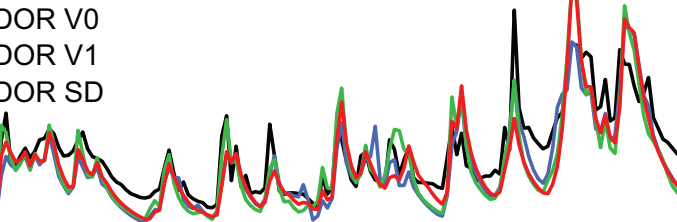

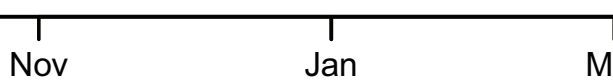

Mar May

$$
\text { c) }
$$

$$
- \text { Obs }
$$

\section{Year 2000}

May

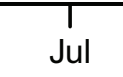

Sep

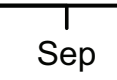

$\infty$ 


\section{S3 Model comparison over evaluation periods}

This section details the performance of MORDOR V0, V1 and V2 over the evaluation periods P1 and P2. For the three considered models, tables S4 and S5 show the values of the individual associated evaluation metrics (NSE(Q), NSE(Qsea), NSE(dQ), NSE(FDC) and NSE(Qlow)) for all the catchments over the evaluation period P1 (table S4) and the evaluation period P2 (table S5). The following figures show, for the 50 catchments, the observed hydrograph (year by year) and those modeled by MORDOR V0, V1 and SD (evaluation model). 
Table S4: The values of the individual associated evaluation metrics (NSE(Q), NSE(Qsea), NSE(dQ), NSE(FDC) and NSE(Qlow)) for MORDOR V0, V1 and SD for all the catchments over the period P1

\begin{tabular}{|c|c|c|c|c|c|c|c|c|c|c|c|c|c|c|c|c|}
\hline \multirow{2}{*}{ ID } & \multirow{2}{*}{ BV } & \multicolumn{5}{|c|}{ MORDOR VO } & \multicolumn{5}{|c|}{ MORDOR V1 } & \multicolumn{5}{|c|}{ MORDOR SD } \\
\hline & & NSE(Q) & NSE(Qsea) & NSE(dQ) & NSE(FDC) & NSE(Qlow) & NSE(Q) & NSE(Qsea) & NSE(dQ) & NSE(FDC) & NSE(Qlow) & NSE(Q) & NSE(Qsea) & NSE(dQ) & NSE(FDC) & NSE(Qlow) \\
\hline 1 & Agout@Fraisse & 0.838 & 0.849 & 0.795 & 0.747 & 0.889 & 0.925 & 0.906 & 0.889 & 0.805 & 0.917 & 0.911 & 0.896 & 0.902 & 0.813 & 0.917 \\
\hline 2 & Agout@LaRaviege & 0.342 & 0.483 & 0.452 & 0.237 & 0.701 & 0.699 & 0.725 & 0.633 & 0.406 & 0.786 & 0.706 & 0.727 & 0.693 & 0.499 & 0.789 \\
\hline 3 & Allier@Poutes & 0.759 & 0.842 & 0.818 & 0.273 & 0.752 & 0.926 & 0.915 & 0.825 & 0.582 & 0.851 & 0.888 & 0.913 & 0.868 & 0.674 & 0.880 \\
\hline 4 & Ardeche@Sauze & 0.937 & 0.935 & 0.858 & 0.875 & 0.883 & 0.938 & 0.959 & 0.860 & 0.909 & 0.927 & 0.923 & 0.954 & 0.855 & 0.896 & 0.933 \\
\hline 5 & Arn@Taillades & 0.936 & 0.957 & 0.891 & 0.848 & 0.874 & 0.939 & 0.974 & 0.930 & 0.905 & 0.957 & 0.920 & 0.972 & 0.935 & 0.930 & 0.958 \\
\hline 6 & Arve@Arthaz & 0.907 & 0.907 & 0.796 & 0.873 & 0.882 & 0.907 & 0.882 & 0.826 & 0.879 & 0.880 & 0.905 & 0.906 & 0.839 & 0.898 & 0.876 \\
\hline 7 & Behine@LaPoutroie & 0.737 & 0.639 & 0.516 & -0.341 & 0.662 & 0.564 & 0.490 & 0.656 & 0.262 & 0.641 & 0.567 & 0.656 & 0.546 & 0.368 & 0.631 \\
\hline 8 & Breze@Meyrueis & 0.889 & 0.802 & 0.442 & 0.282 & 0.779 & 0.860 & 0.840 & 0.602 & 0.602 & 0.857 & 0.863 & 0.864 & 0.553 & 0.777 & 0.874 \\
\hline 9 & Bromme@Brommat & 0.948 & 0.982 & 0.923 & 0.967 & 0.831 & 0.984 & 0.986 & 0.944 & 0.965 & 0.919 & 0.965 & 0.985 & 0.971 & 0.989 & 0.922 \\
\hline 10 & Ceze@Bagnols & 0.913 & 0.972 & 0.914 & 0.883 & 0.915 & 0.970 & 0.990 & 0.907 & 0.852 & 0.954 & 0.944 & 0.982 & 0.938 & 0.930 & 0.957 \\
\hline 11 & Chassezac@SteMarguerite & 0.898 & 0.866 & 0.821 & 0.656 & 0.879 & 0.916 & 0.903 & 0.868 & 0.798 & 0.895 & 0.908 & 0.890 & 0.879 & 0.790 & 0.907 \\
\hline 12 & Creuse@Age & 0.630 & 0.577 & 0.235 & 0.362 & 0.192 & 0.801 & 0.572 & 0.417 & 0.550 & 0.586 & 0.791 & 0.563 & 0.608 & 0.540 & 0.673 \\
\hline 13 & Dordogne@Bort & 0.867 & 0.821 & 0.933 & 0.657 & 0.760 & 0.886 & 0.845 & 0.806 & 0.637 & 0.804 & 0.865 & 0.826 & 0.798 & 0.661 & 0.829 \\
\hline 14 & Doubs@Brenet & 0.851 & 0.945 & 0.944 & 0.549 & 0.932 & 0.972 & 0.970 & 0.951 & 0.924 & 0.982 & 0.975 & 0.971 & 0.942 & 0.912 & 0.978 \\
\hline 15 & Doubs@Neublans & 0.902 & 0.968 & 0.899 & 0.674 & 0.920 & 0.952 & 0.980 & 0.934 & 0.857 & 0.975 & 0.952 & 0.980 & 0.929 & 0.859 & 0.975 \\
\hline 16 & Drac@Sautet & 0.843 & 0.828 & 0.792 & 0.815 & 0.783 & 0.907 & 0.873 & 0.837 & 0.808 & 0.896 & 0.918 & 0.839 & 0.871 & 0.811 & 0.888 \\
\hline 17 & Durance@Clapiere & 0.631 & 0.597 & 0.459 & 0.616 & 0.268 & 0.801 & 0.640 & 0.540 & 0.628 & 0.867 & 0.842 & 0.491 & 0.699 & 0.578 & 0.844 \\
\hline 18 & Eyrieux@Pontpierre & 0.748 & 0.756 & 0.874 & 0.701 & 0.706 & 0.802 & 0.812 & 0.835 & 0.489 & 0.827 & 0.829 & 0.756 & 0.868 & 0.433 & 0.678 \\
\hline 19 & Gage@Gagell & 0.934 & 0.888 & 0.912 & 0.818 & 0.764 & 0.966 & 0.945 & 0.969 & 0.903 & 0.950 & 0.971 & 0.933 & 0.962 & 0.913 & 0.942 \\
\hline 20 & Gardon@Corbes & 0.953 & 0.969 & 0.952 & 0.873 & 0.886 & 0.970 & 0.972 & 0.979 & 0.940 & 0.934 & 0.968 & 0.969 & 0.977 & 0.950 & 0.943 \\
\hline 21 & Gardon@Generargues & 0.803 & 0.871 & 0.840 & 0.863 & 0.798 & 0.854 & 0.914 & 0.926 & 0.880 & 0.776 & 0.870 & 0.904 & 0.921 & 0.884 & 0.767 \\
\hline 22 & GaveEstaube@Gloriettes & 0.701 & 0.666 & 0.477 & 0.580 & 0.408 & 0.480 & 0.685 & 0.729 & 0.607 & 0.339 & 0.483 & 0.644 & 0.703 & 0.670 & 0.293 \\
\hline 23 & Goussant@PontRolland & 0.849 & 0.773 & 0.634 & 0.850 & 0.487 & 0.784 & 0.825 & 0.815 & 0.886 & 0.509 & 0.904 & 0.813 & 0.829 & 0.901 & 0.674 \\
\hline 24 & Loire@Basset & 0.909 & 0.896 & 0.940 & 0.973 & 0.825 & 0.964 & 0.980 & 0.905 & 0.979 & 0.940 & 0.963 & 0.974 & 0.930 & 0.980 & 0.940 \\
\hline 25 & Loire@LaPalisse & 0.871 & 0.960 & 0.910 & 0.964 & 0.877 & 0.959 & 0.988 & 0.869 & 0.970 & 0.953 & 0.952 & 0.984 & 0.902 & 0.969 & 0.952 \\
\hline 26 & Lot@Castelnau & 0.865 & 0.811 & 0.879 & 0.866 & 0.384 & 0.859 & 0.900 & 0.901 & 0.851 & 0.830 & 0.866 & 0.919 & 0.901 & 0.859 & 0.904 \\
\hline 27 & Mimente@Florac & 0.618 & 0.391 & 0.504 & 0.688 & -0.310 & 0.684 & 0.634 & 0.640 & 0.686 & 0.457 & 0.736 & 0.666 & 0.616 & 0.627 & 0.774 \\
\hline 28 & Montane@Eyrein & 0.757 & 0.744 & 0.914 & 0.644 & 0.898 & 0.863 & 0.702 & 0.908 & 0.630 & 0.478 & 0.877 & 0.830 & 0.903 & 0.761 & 0.685 \\
\hline 29 & Oriege@Campauleil & 0.958 & 0.977 & 0.898 & 0.928 & 0.899 & 0.957 & 0.981 & 0.965 & 0.967 & 0.895 & 0.956 & 0.967 & 0.965 & 0.968 & 0.913 \\
\hline 30 & Ouveze@Bedarrides & 0.948 & 0.955 & 0.890 & 0.941 & 0.560 & 0.951 & 0.964 & 0.955 & 0.948 & 0.859 & 0.953 & 0.960 & 0.947 & 0.951 & 0.884 \\
\hline 31 & Rizzanese@Barrage & 0.880 & 0.851 & 0.880 & 0.811 & 0.857 & 0.838 & 0.873 & 0.915 & 0.882 & 0.878 & 0.799 & 0.939 & 0.906 & 0.882 & 0.876 \\
\hline 32 & Romanche@Chambon & 0.574 & 0.209 & 0.620 & 0.605 & 0.460 & 0.569 & 0.366 & 0.709 & 0.535 & 0.601 & 0.356 & 0.528 & 0.693 & 0.541 & 0.591 \\
\hline 33 & Roya@Breil & 0.457 & 0.716 & 0.674 & 0.342 & 0.898 & 0.804 & 0.577 & 0.890 & 0.584 & 0.906 & 0.803 & 0.589 & 0.874 & 0.657 & 0.908 \\
\hline 34 & Salat@Roquefort & 0.885 & 0.985 & 0.898 & 0.890 & 0.934 & 0.937 & 0.982 & 0.945 & 0.912 & 0.961 & 0.925 & 0.984 & 0.947 & 0.920 & 0.964 \\
\hline 35 & Sioule@Fades & 0.900 & 0.860 & 0.969 & 0.770 & 0.961 & 0.936 & 0.898 & 0.950 & 0.840 & 0.974 & 0.937 & 0.923 & 0.954 & 0.859 & 0.967 \\
\hline 36 & Souloise@Infernet & 0.889 & 0.865 & 0.892 & 0.854 & 0.881 & 0.884 & 0.949 & 0.913 & 0.896 & 0.898 & 0.871 & 0.945 & 0.890 & 0.889 & 0.916 \\
\hline 37 & Stura@Lanzo & 0.756 & 0.504 & 0.564 & 0.449 & 0.454 & 0.641 & 0.880 & 0.640 & 0.687 & 0.509 & 0.640 & 0.876 & 0.553 & 0.675 & 0.602 \\
\hline 38 & Tarn@Cocures & 0.789 & 0.763 & 0.889 & 0.648 & 0.788 & 0.868 & 0.845 & 0.888 & 0.875 & 0.569 & 0.824 & 0.855 & 0.873 & 0.849 & 0.628 \\
\hline 39 & Tarn@Millau & 0.875 & 0.941 & 0.979 & 0.872 & 0.976 & 0.946 & 0.930 & 0.983 & 0.970 & 0.985 & 0.940 & 0.928 & 0.984 & 0.962 & 0.979 \\
\hline 40 & Tarn@Montbrun & 0.898 & 0.914 & 0.977 & 0.956 & 0.866 & 0.942 & 0.945 & 0.979 & 0.967 & 0.921 & 0.941 & 0.945 & 0.976 & 0.967 & 0.930 \\
\hline 41 & Tarn@Pinet & 0.829 & 0.881 & 0.764 & 0.900 & 0.846 & 0.882 & 0.888 & 0.812 & 0.934 & 0.873 & 0.860 & 0.879 & 0.806 & 0.933 & 0.874 \\
\hline 42 & Tarnon@Florac & 0.555 & 0.663 & 0.203 & 0.786 & 0.601 & 0.605 & 0.657 & 0.138 & 0.904 & 0.636 & 0.613 & 0.679 & -0.051 & 0.893 & 0.643 \\
\hline 43 & Taurion@RocheTalamie & 0.827 & 0.682 & 0.481 & 0.830 & 0.688 & 0.803 & 0.436 & 0.135 & 0.856 & 0.817 & 0.785 & 0.336 & 0.310 & 0.843 & 0.639 \\
\hline 44 & Tech@Reynes & 0.905 & 0.911 & 0.971 & 0.938 & 0.930 & 0.934 & 0.943 & 0.976 & 0.968 & 0.975 & 0.915 & 0.916 & 0.972 & 0.964 & 0.975 \\
\hline 45 & Tet@Vinca & 0.931 & 0.907 & 0.913 & 0.962 & 0.918 & 0.957 & 0.920 & 0.951 & 0.948 & 0.964 & 0.933 & 0.885 & 0.935 & 0.944 & 0.967 \\
\hline 46 & Tinee@PontLune & 0.825 & 0.843 & 0.794 & 0.868 & 0.869 & 0.853 & 0.887 & 0.886 & 0.927 & 0.906 & 0.857 & 0.879 & 0.888 & 0.924 & 0.927 \\
\hline 47 & Truyere@Grandval & 0.572 & 0.578 & -0.018 & 0.667 & 0.584 & 0.673 & 0.695 & 0.584 & 0.770 & 0.574 & 0.719 & 0.674 & 0.520 & 0.770 & 0.636 \\
\hline 48 & Ubaye@RocheRousse & 0.611 & 0.568 & 0.224 & 0.862 & 0.771 & 0.676 & 0.747 & 0.709 & 0.907 & 0.909 & 0.314 & 0.769 & 0.780 & 0.903 & 0.900 \\
\hline 49 & Vence@Francheville & 0.756 & 0.846 & 0.868 & 0.906 & 0.935 & 0.955 & 0.869 & 0.963 & 0.964 & 0.971 & 0.957 & 0.899 & 0.953 & 0.967 & 0.965 \\
\hline 50 & Vienne@Bussy & 0.745 & 0.897 & 0.832 & 0.947 & 0.941 & 0.958 & 0.910 & 0.928 & 0.968 & 0.977 & 0.946 & 0.940 & 0.918 & 0.975 & 0.976 \\
\hline
\end{tabular}


Table S5: The values of the individual associated evaluation metrics (NSE(Q), NSE(Qsea), NSE(dQ), NSE(FDC) and NSE(Qlow)) for MORDOR V0, V1 and SD for all the catchments over the period P2

\begin{tabular}{|c|c|c|c|c|c|c|c|c|c|c|c|c|c|c|c|c|}
\hline \multirow{2}{*}{ ID } & \multirow{2}{*}{ BV } & \multicolumn{5}{|c|}{ MORDOR VO } & \multicolumn{5}{|c|}{ MORDOR V1 } & \multicolumn{5}{|c|}{ MORDOR SD } \\
\hline & & NSE(Q) & NSE(Qsea) & NSE(dQ) & NSE(FDC) & NSE(Qlow) & NSE(Q) & NSE(Qsea) & NSE(dQ) & NSE(FDC) & NSE(Qlow) & NSE(Q) & NSE(Qsea) & NSE(dQ) & NSE(FDC) & NSE(Qlow) \\
\hline 1 & Agout@Fraisse & 0.904 & 0.899 & 0.867 & 0.864 & 0.916 & 0.965 & 0.949 & 0.943 & 0.873 & 0.960 & 0.958 & 0.929 & 0.948 & 0.870 & 0.949 \\
\hline 2 & Agout@LaRaviege & 0.927 & 0.950 & 0.937 & 0.788 & 0.908 & 0.961 & 0.977 & 0.965 & 0.780 & 0.955 & 0.957 & 0.973 & 0.964 & 0.905 & 0.952 \\
\hline 3 & Allier@Poutes & 0.871 & 0.897 & 0.782 & 0.827 & 0.802 & 0.894 & 0.916 & 0.797 & 0.820 & 0.909 & 0.883 & 0.914 & 0.802 & 0.794 & 0.916 \\
\hline 4 & Ardeche@Sauze & 0.562 & 0.737 & 0.426 & 0.539 & 0.591 & 0.647 & 0.704 & 0.477 & 0.451 & 0.787 & 0.656 & 0.709 & 0.506 & 0.215 & 0.788 \\
\hline 5 & Arn@Taillades & 0.843 & 0.847 & 0.852 & 0.763 & 0.687 & 0.772 & 0.850 & 0.782 & 0.589 & 0.823 & 0.714 & 0.868 & 0.766 & 0.365 & 0.826 \\
\hline 6 & Arve@Arthaz & 0.954 & 0.980 & 0.932 & 0.967 & 0.917 & 0.973 & 0.983 & 0.951 & 0.979 & 0.917 & 0.973 & 0.988 & 0.966 & 0.971 & 0.915 \\
\hline 7 & Behine@LaPoutroie & 0.944 & 0.979 & 0.901 & 0.791 & 0.937 & 0.953 & 0.975 & 0.936 & 0.920 & 0.950 & 0.961 & 0.980 & 0.962 & 0.910 & 0.946 \\
\hline 8 & Breze@Meyrueis & 0.845 & 0.893 & 0.797 & 0.854 & 0.796 & 0.947 & 0.925 & 0.813 & 0.880 & 0.890 & 0.939 & 0.925 & 0.847 & 0.917 & 0.888 \\
\hline 9 & Bromme@Brommat & 0.393 & 0.660 & 0.538 & 0.263 & 0.356 & 0.862 & 0.562 & 0.586 & 0.030 & 0.595 & 0.870 & 0.509 & 0.611 & 0.365 & 0.593 \\
\hline 10 & Ceze@Bagnols & 0.759 & 0.832 & 0.418 & 0.710 & 0.814 & 0.905 & 0.799 & 0.327 & 0.451 & 0.691 & 0.872 & 0.826 & 0.589 & 0.760 & 0.830 \\
\hline 11 & Chassezac@SteMarguerite & 0.937 & 0.927 & 0.889 & 0.758 & 0.934 & 0.926 & 0.974 & 0.944 & 0.835 & 0.984 & 0.912 & 0.975 & 0.944 & 0.841 & 0.986 \\
\hline 12 & Creuse@Age & 0.919 & 0.937 & 0.877 & 0.739 & 0.957 & 0.943 & 0.979 & 0.924 & 0.828 & 0.972 & 0.952 & 0.979 & 0.919 & 0.890 & 0.977 \\
\hline 13 & Dordogne@Bort & 0.869 & 0.871 & 0.862 & 0.584 & 0.781 & 0.951 & 0.902 & 0.910 & 0.860 & 0.915 & 0.953 & 0.912 & 0.897 & 0.857 & 0.917 \\
\hline 14 & Doubs@Brenet & 0.646 & 0.506 & 0.575 & 0.106 & -0.038 & 0.818 & 0.546 & 0.574 & 0.599 & 0.497 & 0.823 & 0.610 & 0.512 & 0.621 & 0.570 \\
\hline 15 & Doubs@Neublans & 0.887 & 0.814 & 0.764 & 0.586 & 0.749 & 0.869 & 0.896 & 0.890 & 0.610 & 0.884 & 0.895 & 0.888 & 0.871 & 0.641 & 0.888 \\
\hline 16 & Drac@Sautet & 0.928 & 0.910 & 0.905 & 0.880 & 0.847 & 0.954 & 0.953 & 0.949 & 0.898 & 0.896 & 0.956 & 0.950 & 0.954 & 0.893 & 0.874 \\
\hline 17 & Durance@Clapiere & 0.946 & 0.953 & 0.906 & 0.901 & 0.815 & 0.970 & 0.980 & 0.973 & 0.949 & 0.938 & 0.966 & 0.978 & 0.964 & 0.946 & 0.907 \\
\hline 18 & Eyrieux@Pontpierre & 0.883 & 0.840 & 0.805 & 0.706 & 0.771 & 0.911 & 0.872 & 0.860 & 0.846 & 0.924 & 0.917 & 0.850 & 0.881 & 0.866 & 0.920 \\
\hline 19 & Gage@Gagell & 0.646 & 0.654 & 0.558 & 0.318 & 0.542 & 0.800 & 0.609 & 0.806 & 0.757 & 0.803 & 0.802 & 0.538 & 0.810 & 0.766 & 0.781 \\
\hline 20 & Gardon@Corbes & 0.825 & 0.846 & 0.811 & -0.121 & 0.816 & 0.753 & 0.866 & 0.836 & 0.745 & 0.772 & 0.829 & 0.865 & 0.882 & 0.824 & 0.818 \\
\hline 21 & Gardon@Generargues & 0.930 & 0.925 & 0.917 & 0.967 & 0.891 & 0.950 & 0.980 & 0.947 & 0.979 & 0.916 & 0.952 & 0.977 & 0.947 & 0.977 & 0.904 \\
\hline 22 & GaveEstaube@Gloriettes & 0.897 & 0.960 & 0.856 & 0.968 & 0.934 & 0.952 & 0.986 & 0.927 & 0.976 & 0.953 & 0.952 & 0.985 & 0.918 & 0.975 & 0.942 \\
\hline 23 & Goussant@PontRolland & 0.784 & 0.858 & 0.897 & 0.849 & 0.683 & 0.869 & 0.934 & 0.853 & 0.883 & 0.846 & 0.857 & 0.936 & 0.886 & 0.891 & 0.854 \\
\hline 24 & Loire@Basset & 0.293 & 0.656 & 0.642 & 0.603 & 0.054 & 0.596 & 0.762 & 0.621 & 0.683 & 0.574 & 0.598 & 0.739 & 0.666 & 0.726 & 0.604 \\
\hline 25 & Loire@LaPalisse & 0.853 & 0.834 & 0.548 & 0.884 & 0.397 & 0.873 & 0.887 & 0.514 & 0.857 & 0.577 & 0.879 & 0.877 & 0.649 & 0.882 & 0.530 \\
\hline 26 & Lot@Castelnau & 0.947 & 0.965 & 0.930 & 0.943 & 0.636 & 0.935 & 0.978 & 0.974 & 0.944 & 0.962 & 0.945 & 0.985 & 0.976 & 0.958 & 0.983 \\
\hline 27 & Mimente@Florac & 0.937 & 0.939 & 0.941 & 0.902 & 0.486 & 0.938 & 0.958 & 0.977 & 0.927 & 0.850 & 0.939 & 0.968 & 0.976 & 0.958 & 0.926 \\
\hline 28 & Montane@Eyrein & 0.886 & 0.889 & 0.794 & 0.774 & 0.679 & 0.883 & 0.896 & 0.920 & 0.896 & 0.801 & 0.880 & 0.902 & 0.901 & 0.923 & 0.813 \\
\hline 29 & Oriege@Campauleil & 0.652 & 0.598 & 0.462 & 0.186 & 0.350 & 0.592 & 0.615 & 0.771 & 0.614 & 0.515 & 0.602 & 0.683 & 0.713 & 0.766 & 0.529 \\
\hline 30 & Ouveze@Bedarrides & 0.868 & 0.808 & 0.833 & 0.771 & 0.278 & 0.804 & 0.885 & 0.883 & 0.773 & 0.750 & 0.815 & 0.881 & 0.894 & 0.796 & 0.815 \\
\hline 31 & Rizzanese@Barrage & 0.917 & 0.976 & 0.921 & 0.905 & 0.934 & 0.887 & 0.971 & 0.934 & 0.959 & 0.972 & 0.866 & 0.990 & 0.928 & 0.953 & 0.970 \\
\hline 32 & Romanche@Chambon & 0.865 & 0.847 & 0.924 & 0.808 & 0.957 & 0.918 & 0.817 & 0.974 & 0.915 & 0.982 & 0.890 & 0.922 & 0.974 & 0.915 & 0.979 \\
\hline 33 & Roya@Breil & 0.811 & 0.838 & 0.873 & 0.783 & 0.829 & 0.896 & 0.904 & 0.918 & 0.833 & 0.859 & 0.886 & 0.905 & 0.922 & 0.856 & 0.869 \\
\hline 34 & Salat@Roquefort & 0.284 & -0.057 & 0.547 & 0.353 & 0.543 & 0.648 & -0.135 & 0.694 & 0.532 & 0.479 & 0.635 & 0.156 & 0.702 & 0.586 & 0.435 \\
\hline 35 & Sioule@Fades & 0.847 & 0.684 & 0.889 & 0.452 & 0.869 & 0.617 & 0.760 & 0.891 & 0.766 & 0.883 & 0.546 & 0.784 & 0.894 & 0.547 & 0.885 \\
\hline 36 & Souloise@Infernet & 0.928 & 0.897 & 0.982 & 0.930 & 0.970 & 0.928 & 0.964 & 0.983 & 0.919 & 0.978 & 0.912 & 0.961 & 0.975 & 0.911 & 0.986 \\
\hline 37 & Stura@Lanzo & 0.909 & 0.936 & 0.974 & 0.900 & 0.910 & 0.939 & 0.943 & 0.980 & 0.961 & 0.920 & 0.933 & 0.942 & 0.975 & 0.956 & 0.937 \\
\hline 38 & Tarn@Cocures & 0.851 & 0.927 & 0.883 & 0.848 & 0.799 & 0.907 & 0.906 & 0.904 & 0.936 & 0.896 & 0.902 & 0.901 & 0.908 & 0.926 & 0.901 \\
\hline 39 & Tarn@Millau & 0.632 & 0.793 & 0.508 & 0.494 & 0.081 & 0.719 & 0.729 & 0.599 & 0.750 & 0.536 & 0.733 & 0.721 & 0.594 & 0.734 & 0.612 \\
\hline 40 & Tarn@Montbrun & 0.768 & 0.723 & 0.858 & 0.861 & 0.658 & 0.824 & 0.866 & 0.861 & 0.610 & 0.693 & 0.835 & 0.838 & 0.864 & 0.620 & 0.682 \\
\hline 41 & Tarn@Pinet & 0.899 & 0.939 & 0.968 & 0.926 & 0.946 & 0.910 & 0.927 & 0.970 & 0.956 & 0.956 & 0.876 & 0.921 & 0.977 & 0.955 & 0.937 \\
\hline 42 & Tarnon@Florac & 0.923 & 0.898 & 0.936 & 0.897 & 0.903 & 0.939 & 0.914 & 0.925 & 0.971 & 0.934 & 0.922 & 0.891 & 0.919 & 0.970 & 0.919 \\
\hline 43 & Taurion@RocheTalamie & 0.875 & 0.867 & 0.804 & 0.901 & 0.808 & 0.870 & 0.883 & 0.809 & 0.924 & 0.926 & 0.858 & 0.862 & 0.785 & 0.917 & 0.926 \\
\hline 44 & Tech@Reynes & 0.596 & 0.631 & 0.026 & 0.853 & 0.579 & 0.588 & 0.610 & 0.284 & 0.812 & 0.717 & 0.560 & 0.581 & 0.313 & 0.815 & 0.698 \\
\hline 45 & Tet@Vinca & 0.787 & 0.321 & 0.382 & 0.780 & 0.713 & 0.859 & 0.776 & 0.351 & 0.873 & 0.683 & 0.876 & 0.704 & 0.456 & 0.837 & 0.721 \\
\hline 46 & Tinee@PontLune & 0.838 & 0.895 & 0.854 & 0.936 & 0.943 & 0.900 & 0.928 & 0.892 & 0.954 & 0.972 & 0.901 & 0.930 & 0.903 & 0.950 & 0.979 \\
\hline 47 & Truyere@Grandval & 0.623 & 0.935 & 0.842 & 0.903 & 0.962 & 0.913 & 0.965 & 0.834 & 0.975 & 0.980 & 0.902 & 0.965 & 0.863 & 0.972 & 0.979 \\
\hline 48 & Ubaye@RocheRousse & 0.707 & 0.759 & 0.823 & 0.895 & 0.872 & 0.945 & 0.811 & 0.936 & 0.924 & 0.868 & 0.951 & 0.844 & 0.927 & 0.931 & 0.864 \\
\hline 49 & Vence@Francheville & 0.386 & 0.384 & 0.390 & 0.655 & 0.556 & 0.931 & 0.494 & 0.774 & 0.779 & 0.614 & 0.935 & 0.582 & 0.740 & 0.788 & 0.525 \\
\hline 50 & Vienne@Bussy & 0.438 & 0.641 & 0.569 & 0.863 & 0.825 & 0.818 & 0.601 & 0.740 & 0.854 & 0.816 & 0.726 & 0.617 & 0.830 & 0.836 & 0.789 \\
\hline
\end{tabular}




\section{Agout@Fraisse}

Year 1986

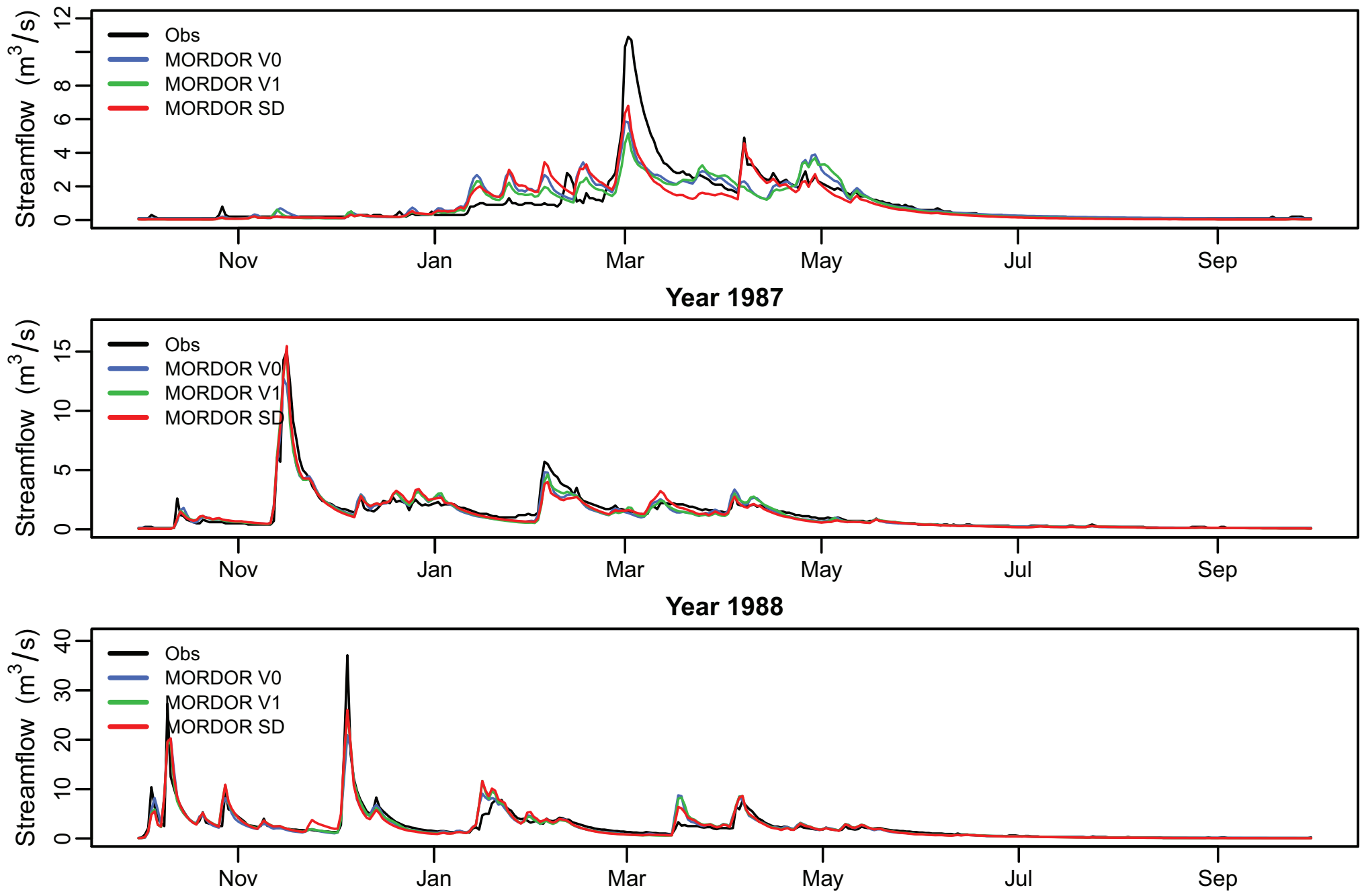




\section{Agout@LaRaviege}

Year 1986

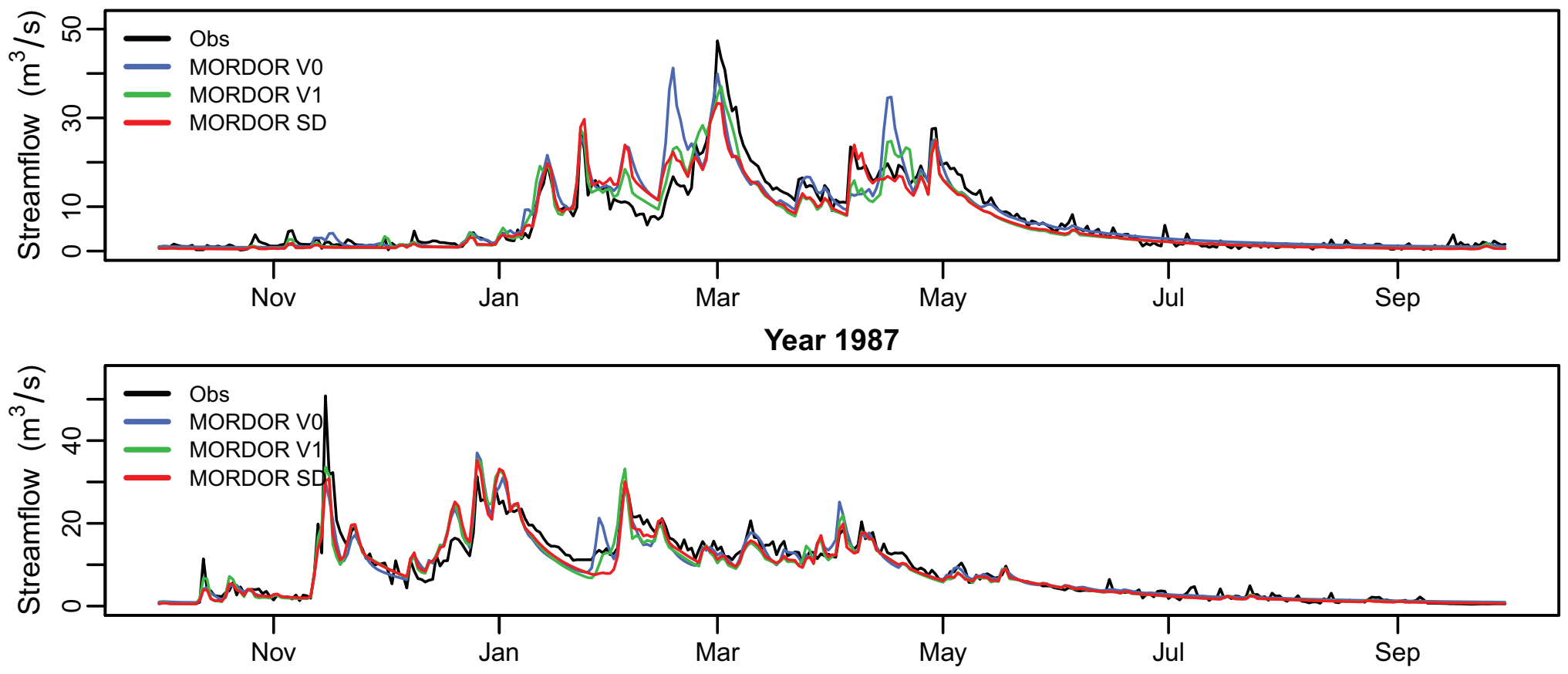


Allier@Poutes

Year 1989

क

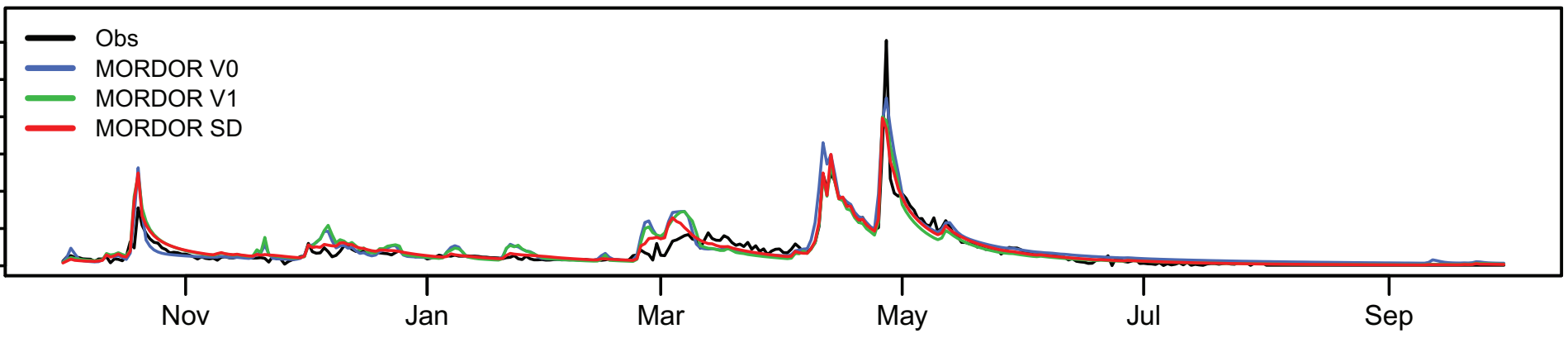

Year 1990
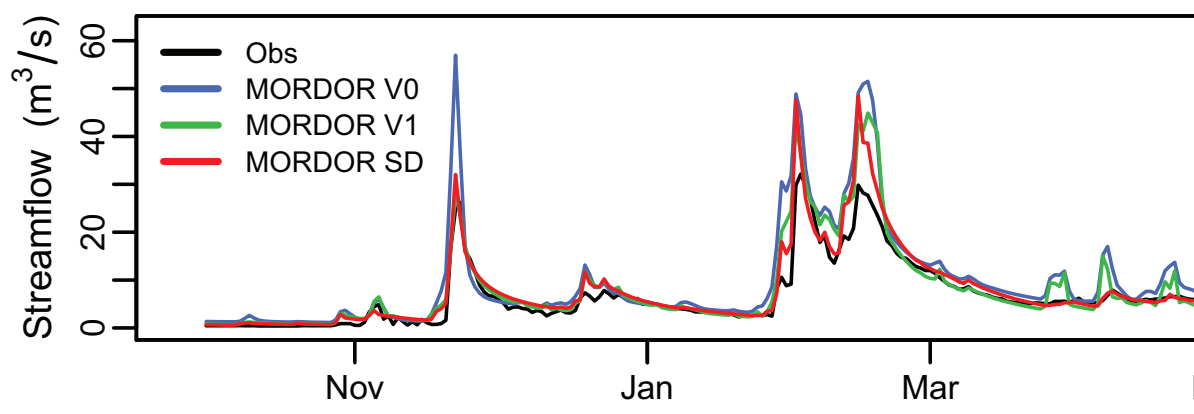

Year 1991
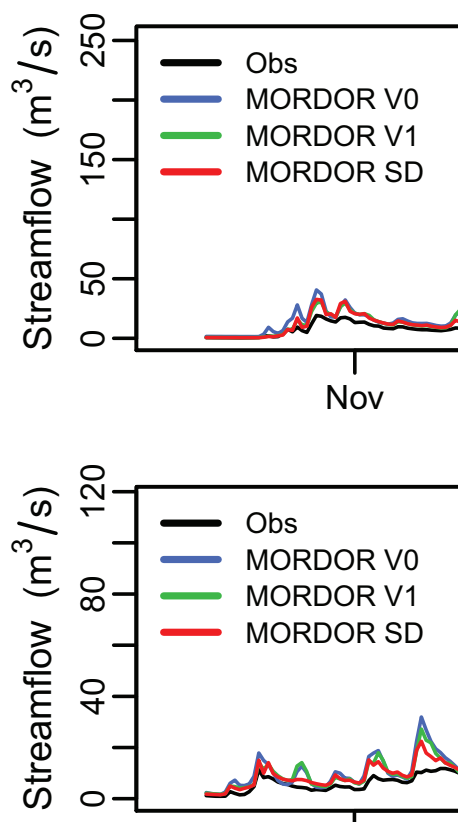

MORDOR V0

$\longrightarrow$ MORDOR SD

政

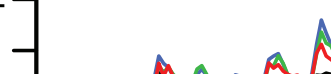

Nov

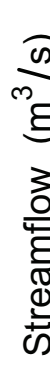

。

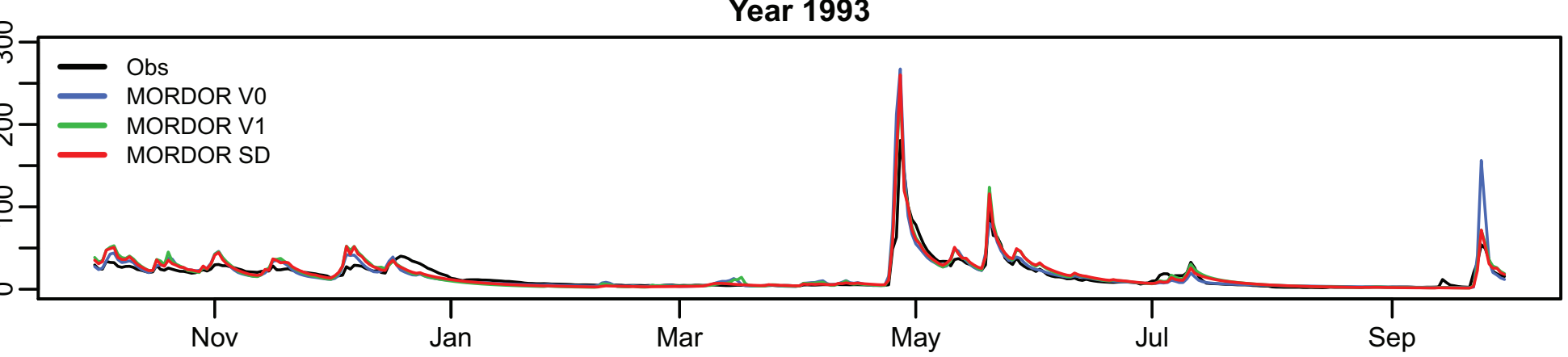

Year 1994

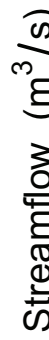

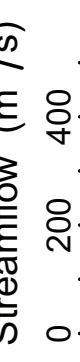
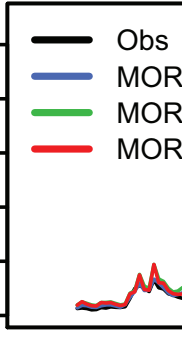

RDOR VO 

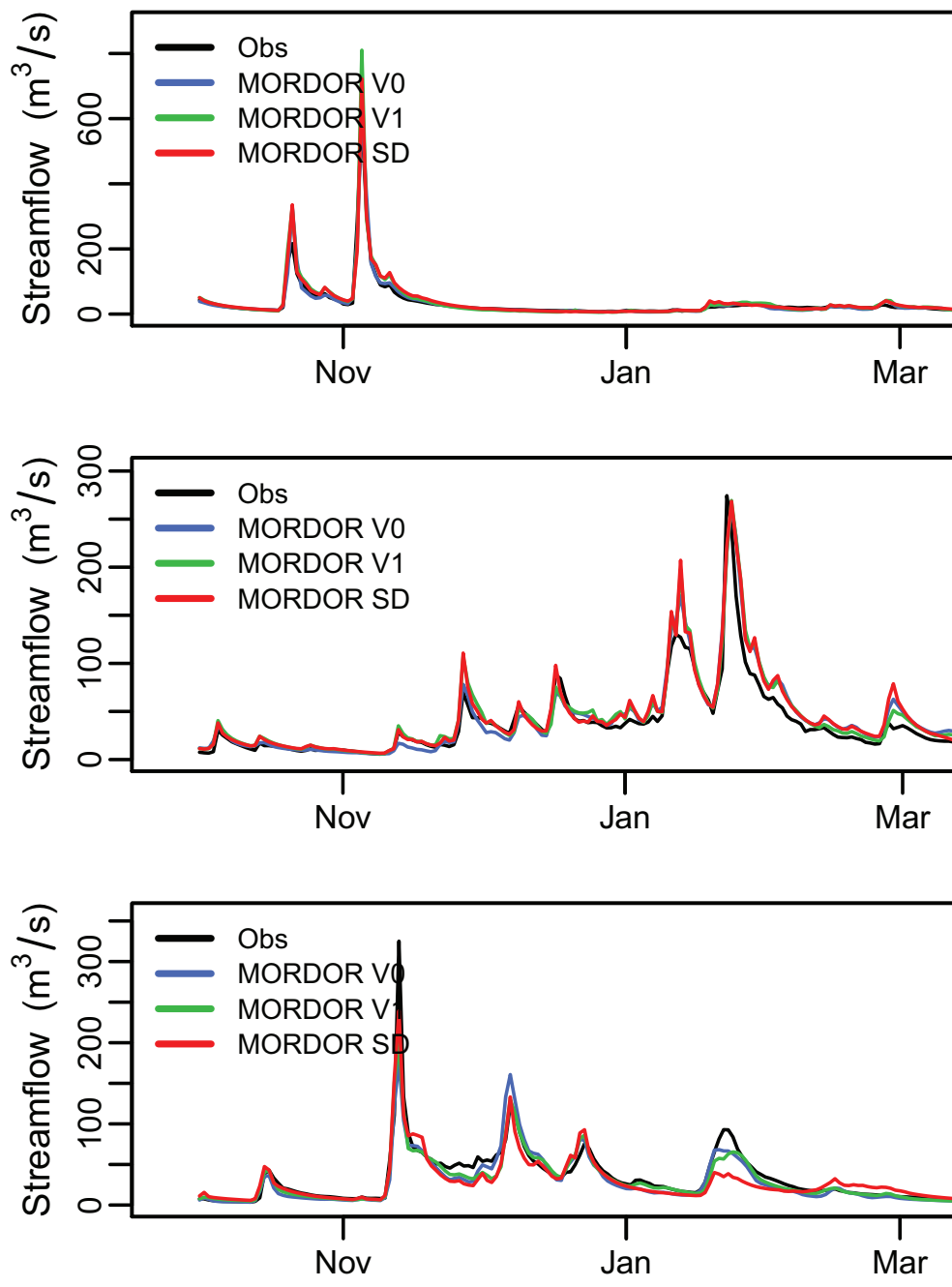

Year 1997

Year 1996

May $\quad$ Jul $\quad$ Sep

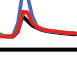




\section{Arn@Taillades}

\section{Year 1994}
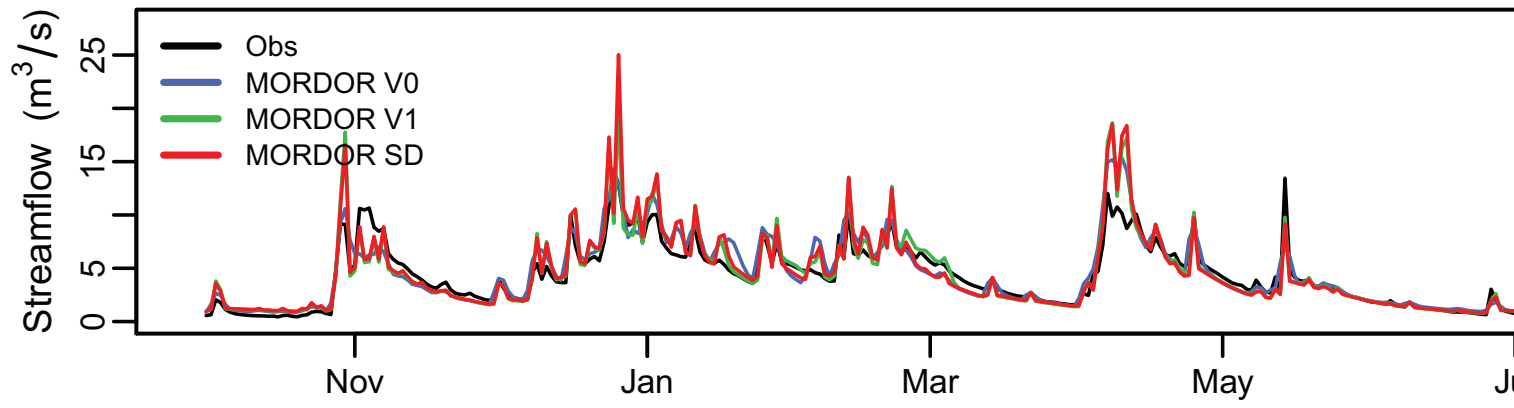

Year 1995

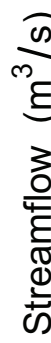

三 Obs

- MORDOR V0

- MORDOR V1

moroos 50

andarberand

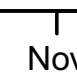

Nov

Jan

Year 1996

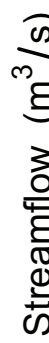

- Obs

- MORDOR VO

- MORDOR SD

ㅇ-
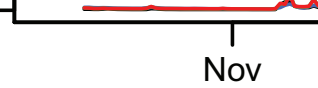

dathow ha

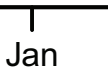

Year 1997

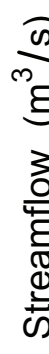

- Ops

- MpRDOR V0

- MORDOR V1
MPRDOR SD

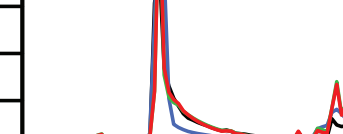

Nov
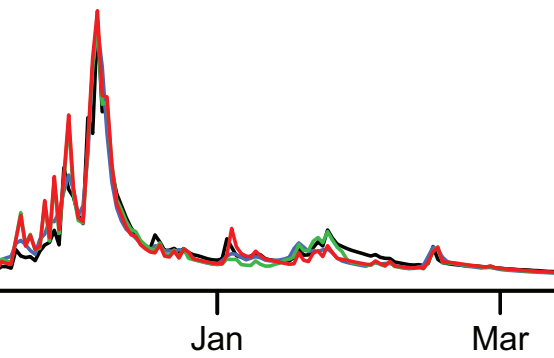

Year 1998

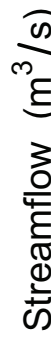

- Obs

- MORDOR V0

OP- MORDOR V1

m- MORDOR SD

宽

o- an

Nov

hand
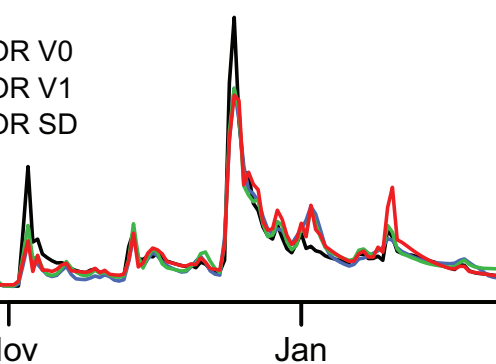

Jan
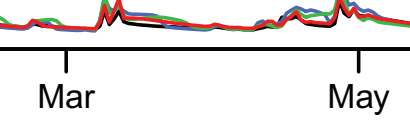

1

Year 1999

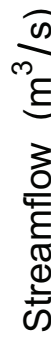

$-\begin{array}{ll}- & \text { Obs } \\ \text { MORDOR V0 } & \text { MORDOR V1 }\end{array}$

으- $=$ MORDOR V1

है

0

Musarasos

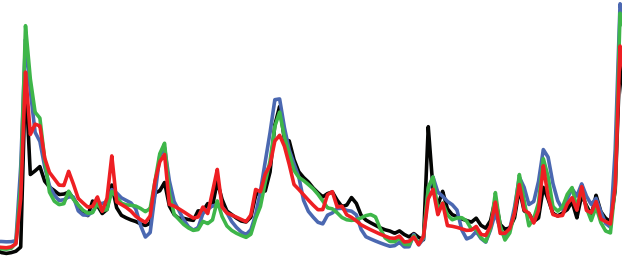




\section{Arn@Taillades}

\section{Year 2000}
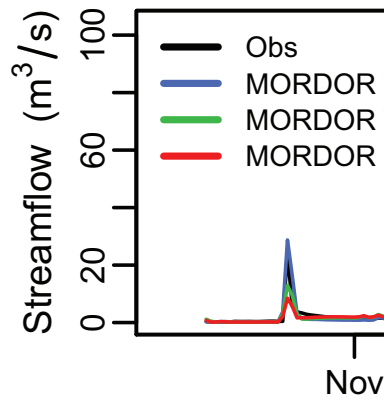

近

dina

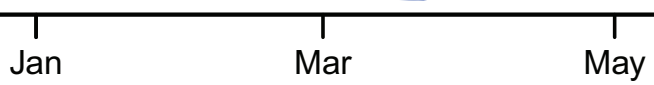

Year 2001

क

\section{$\longrightarrow$ Obs}

$\longrightarrow$ MORDOR V0

MORDOR V1

$\longrightarrow$ MORDOR SD

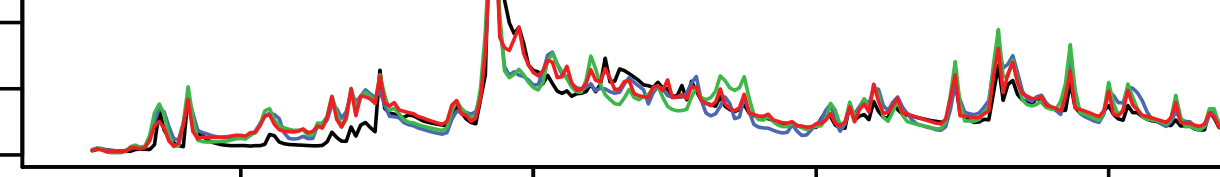
Nov Jan Mar May Jul

Year 2002

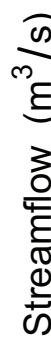

$\sqrt{- \text { Obs }}$

$\stackrel{2}{2}=$ MORDOR V0

MORDOR SD
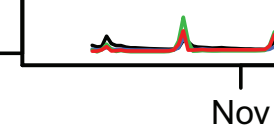

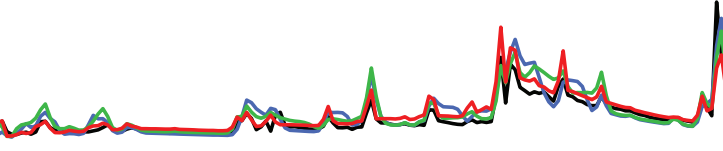

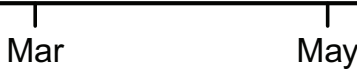

Year 2003

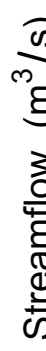

$\begin{array}{ll}- & \text { Obs } \\ - & \text { MORDOR V0 } \\ \text { MORDOR V1 }\end{array}$

MORDOR SD

趂 을

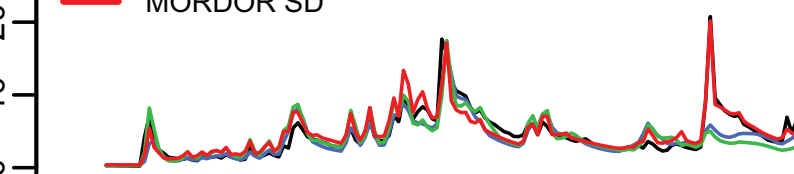

Nov

Jan

Mar

Year 2004

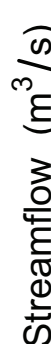

वे
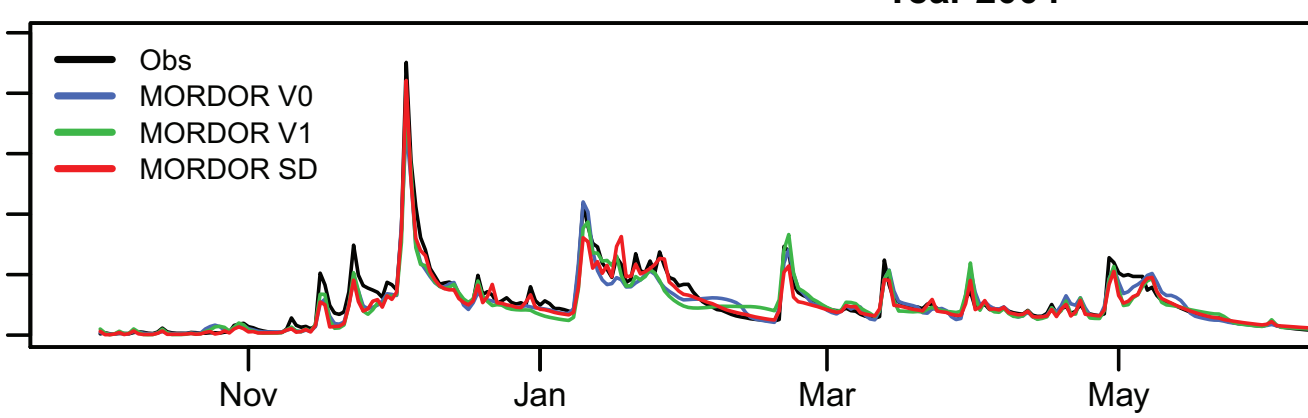

Mar

Year 2005
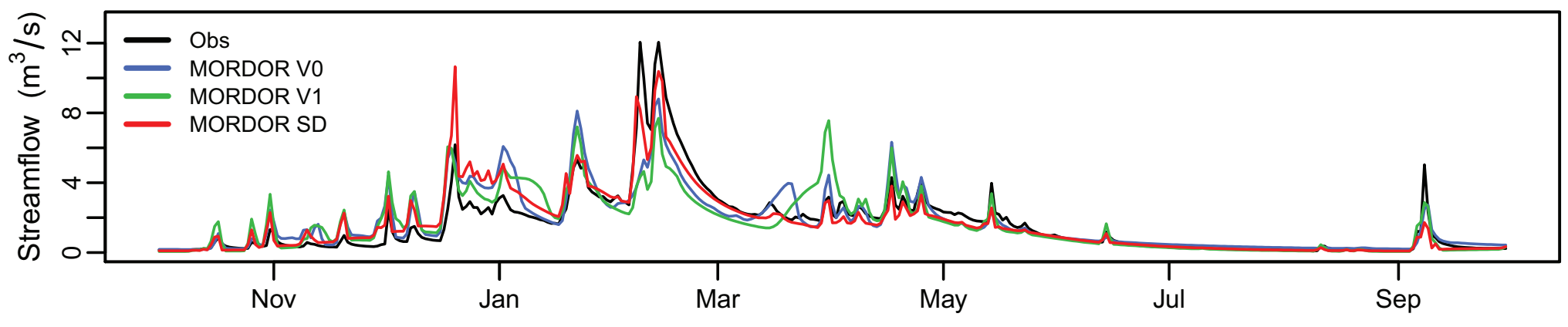


\section{Arn@Taillades}

\section{Year 2006}

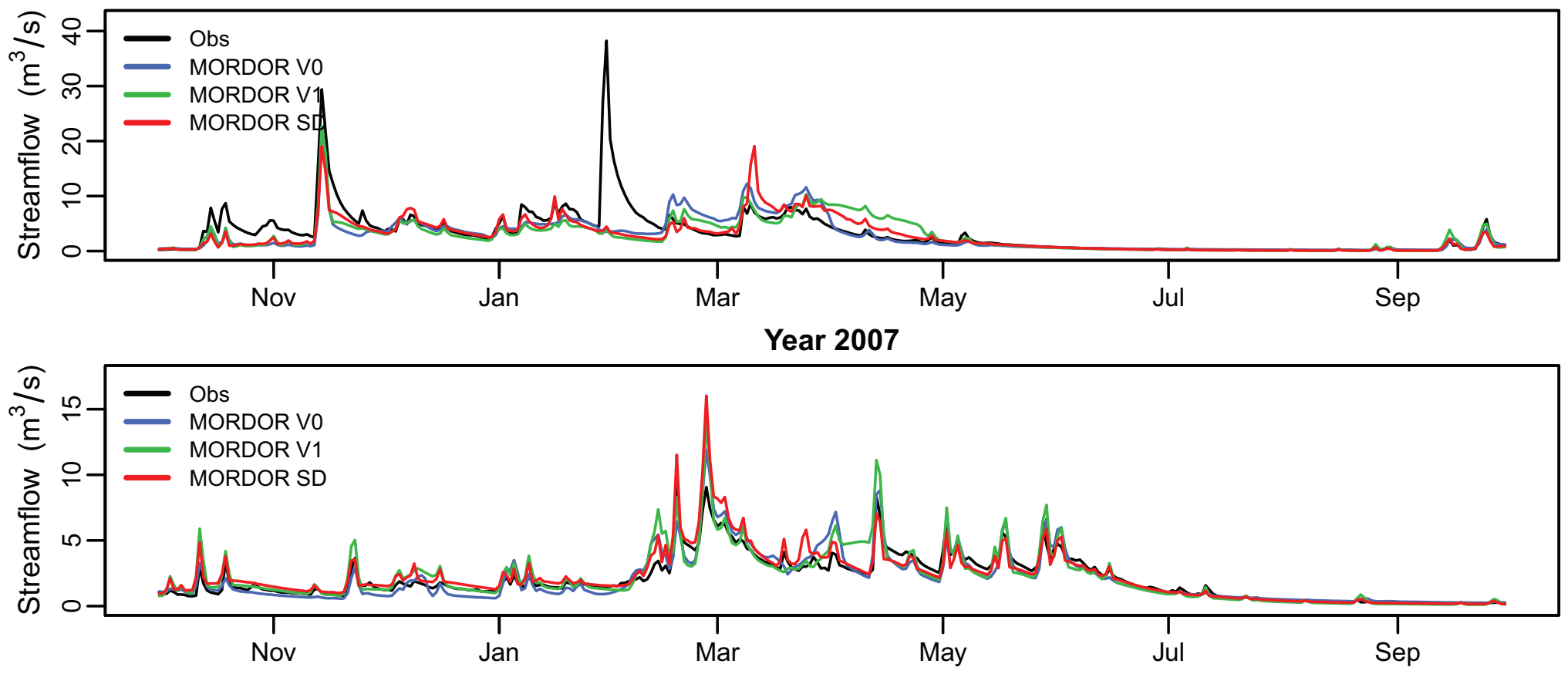


Arve@Arthaz

Year 1999

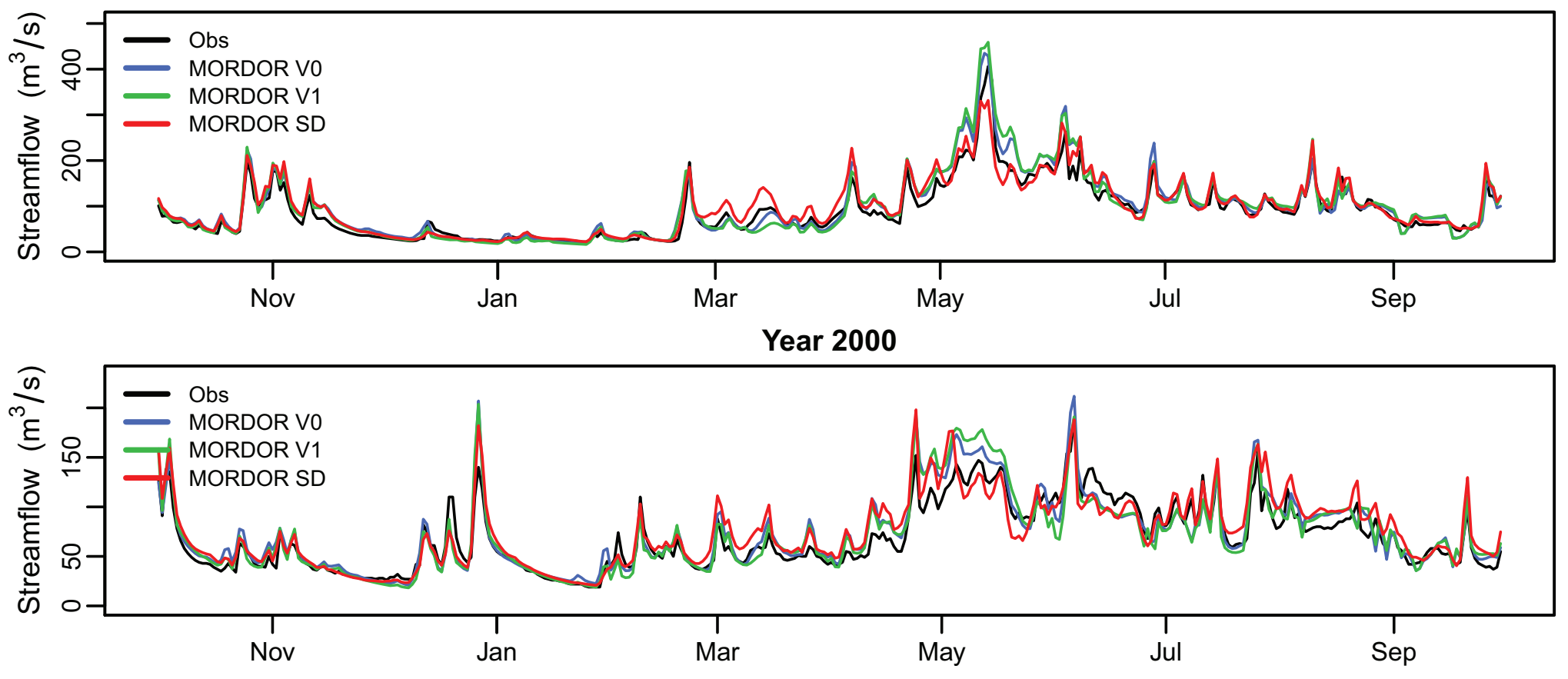




\section{Breze@Meyrueis}

Year 1992
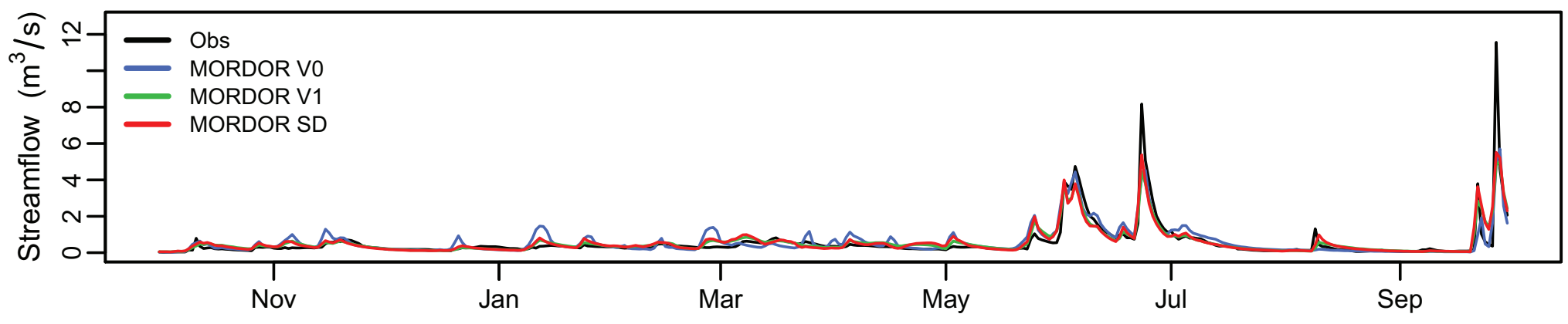

Year 1993
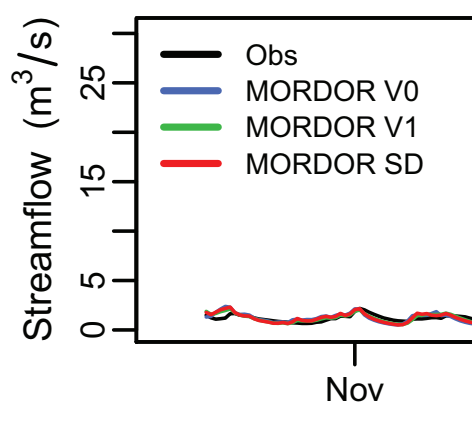

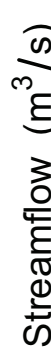

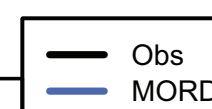

Obs

MORDOR V0

- MORDOR V1

- MORDOR SD

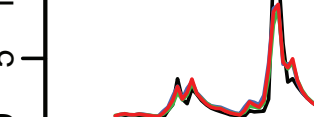

$0-120$

Nov

Jan

Mar

Year 1995
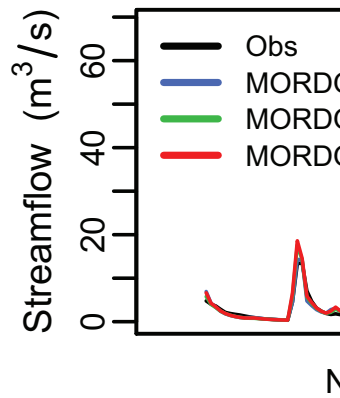

\section{V0}

SD

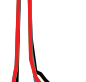

1

Jan

Mar

Year 1994

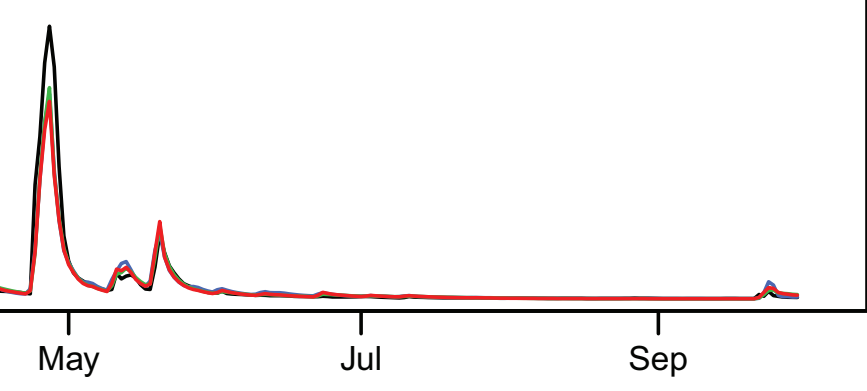

Year 1994 


\section{Breze@Meyrueis}

Year 2004
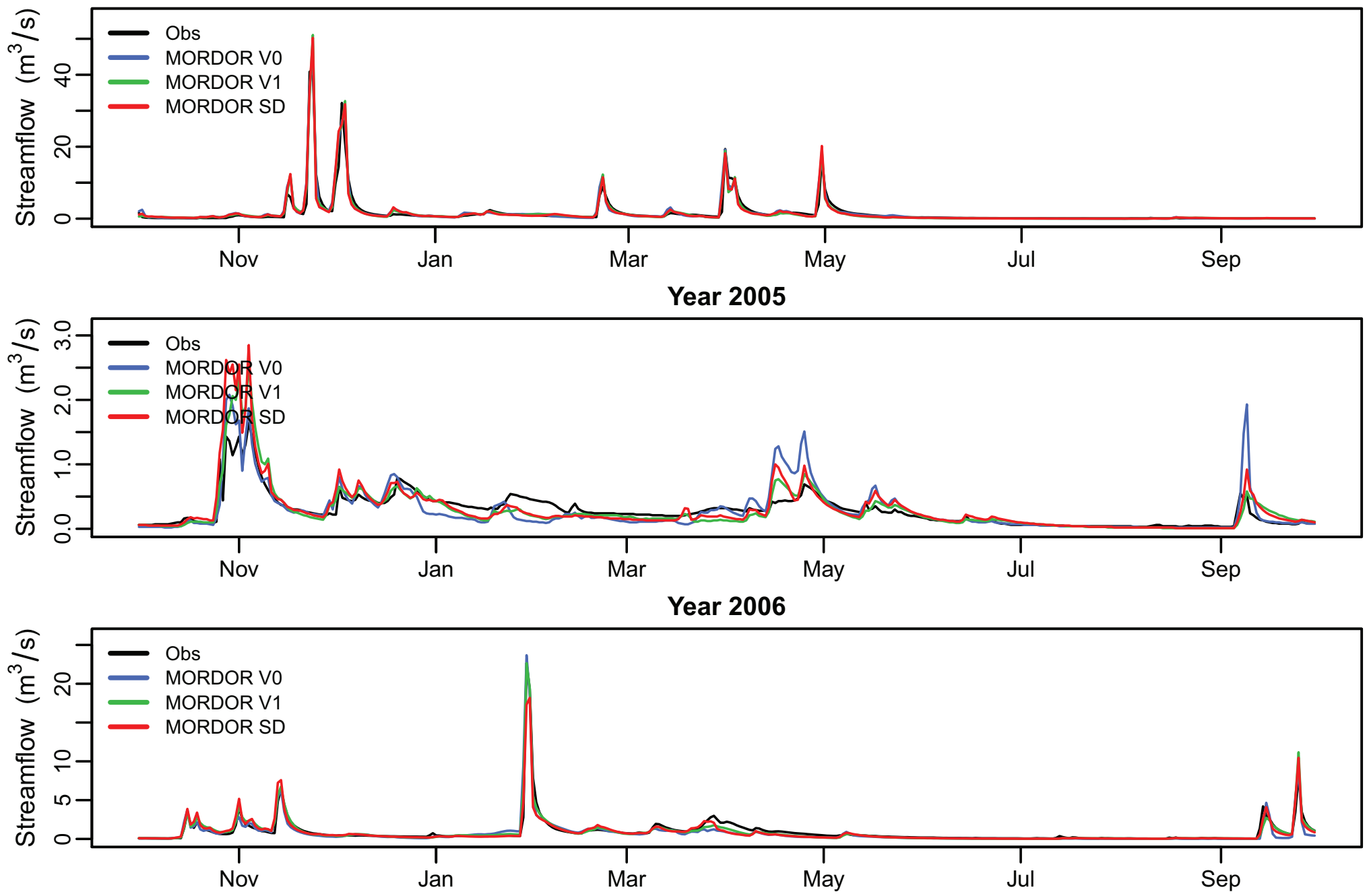
Bromme@Brommat

Year 1990

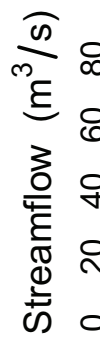

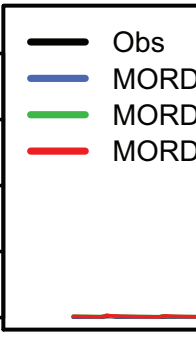

Year 1991

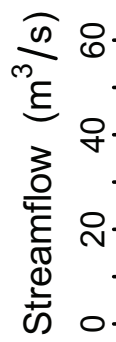

- Obs

- MORDфR V0

- MORDQR V1

MORDQR SD

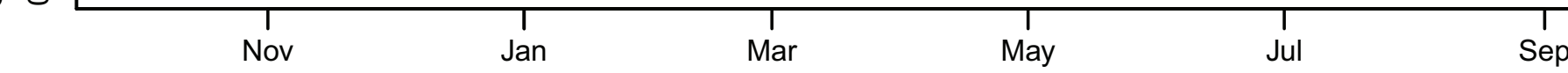

Year 1992

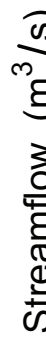

ما

- obs

MORDOR VO

- MORDOR SD

N

Nov

Nov

a

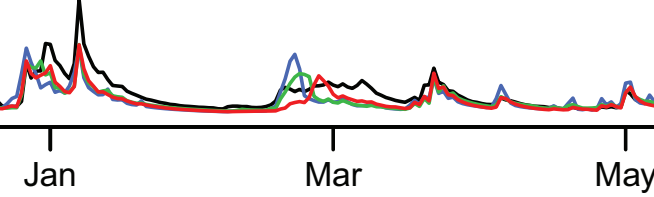

May

Jul

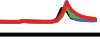

(1)




\section{Ceze@Bagnols}

\section{Year 1995}
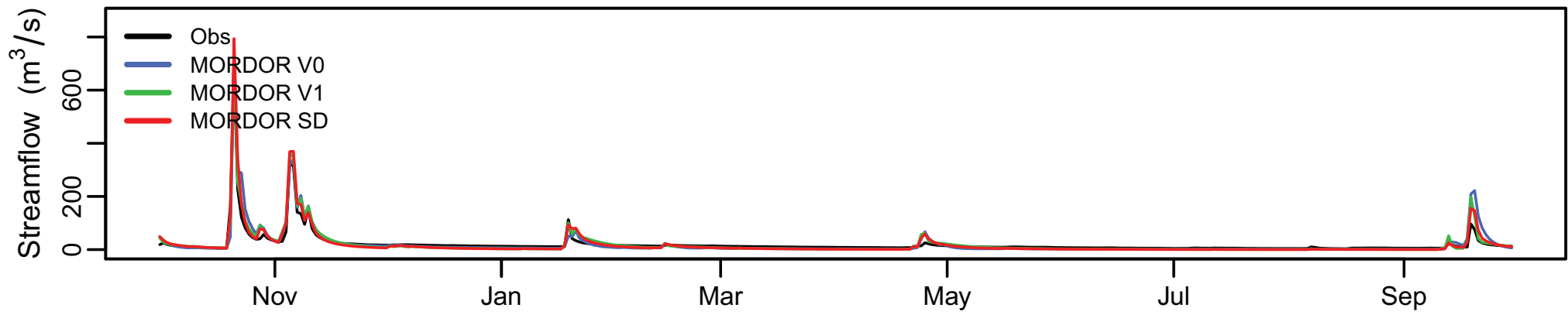

Year 1996
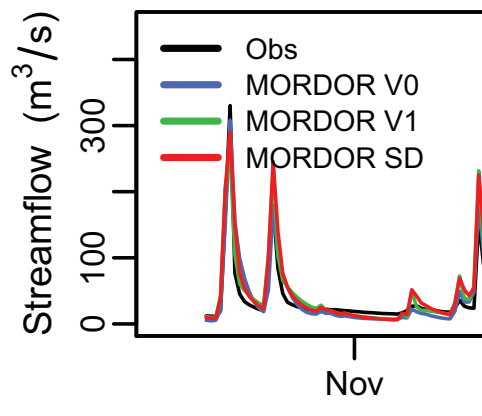

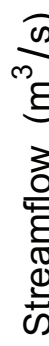
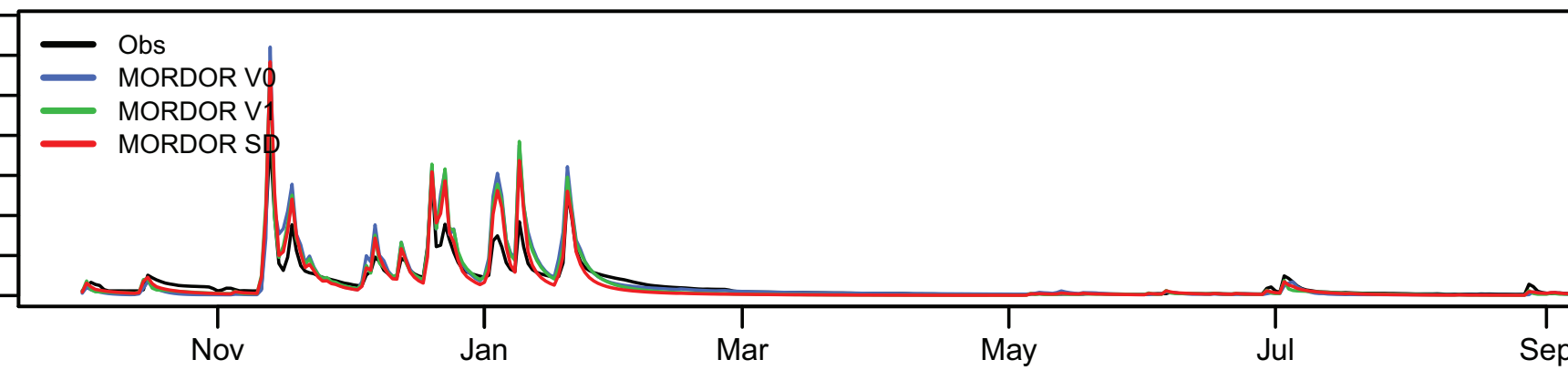

Year 1998
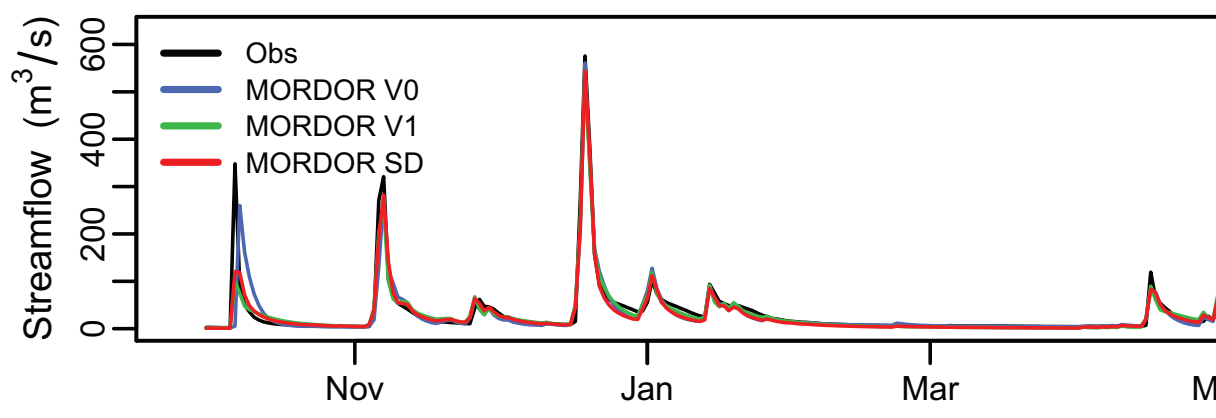

Year 1999

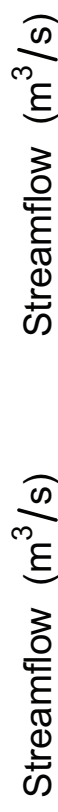

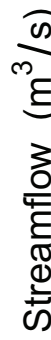

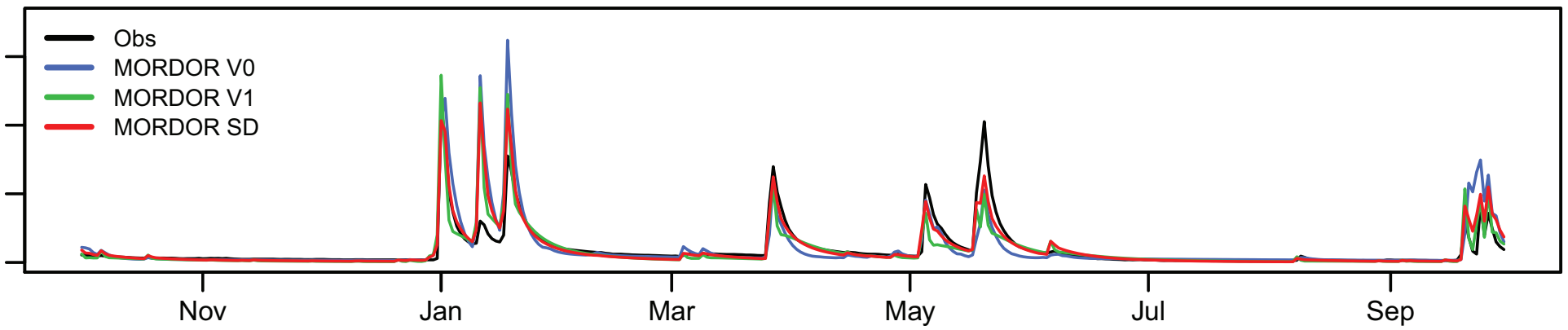

Year 2000
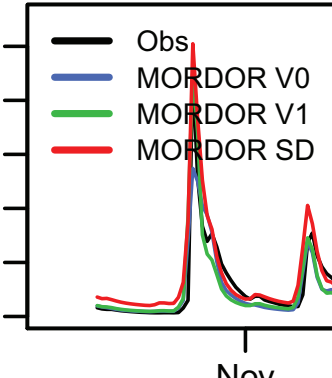

n 


\section{Chassezac@SteMarguerite}

Year 1987

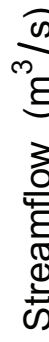

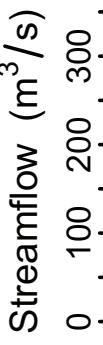

$\longrightarrow$ Obs

MORDOR VO

$\longrightarrow$ MORDOR SD

N

Nov

Jan

Mar

May

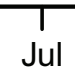

Year 1988

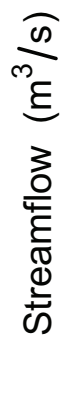

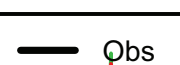

- Obs

MORDOR VO

- MORDOR

h

:- Nh

Nov $\quad$ Jan $\quad$ Mar $\quad$ May $\quad$ Jul Sep

Year 1989

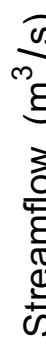

- Obs

- MORDOR V0

- MORDOR V1

○- - MORDOR SD

毫

o- $\alpha$ W
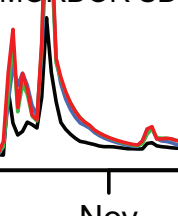

Nov

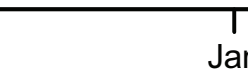

Jan Mar

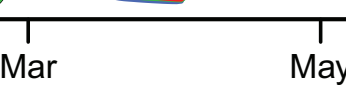

Year 1990

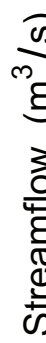

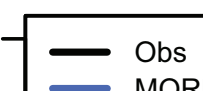

- MORDOR V0

- MORDOR SD

으-

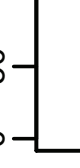
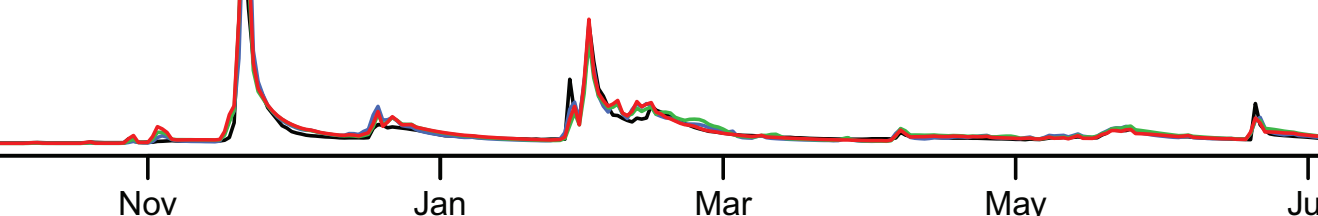

Year 1991

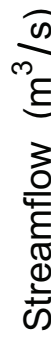

Obs

MORDOR V0

MORDOR V1

$-1-$ MORDOR SD

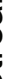
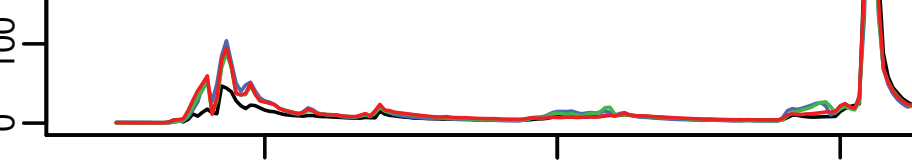

Nov

Jan

Mar

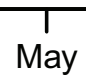

Jul

Year 1992

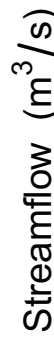

(2)

Obs

- MORDOR V0

MORDOR V1

$-$

MORDOR SD

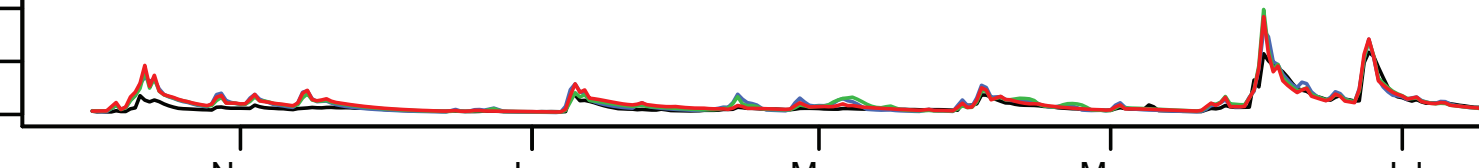




\section{Chassezac@SteMarguerite}

Year 1993

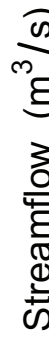

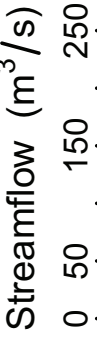

$\longrightarrow$ Obs

$\longrightarrow$ MORDOR V0

$\longrightarrow$ MORDOR V1

MORDOR SD

.

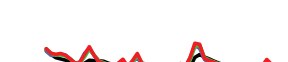
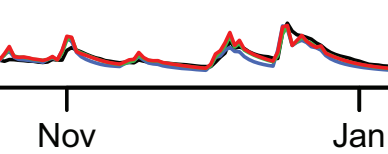

Jan Mar

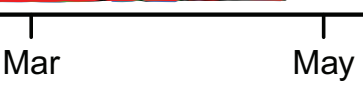

Year 1994
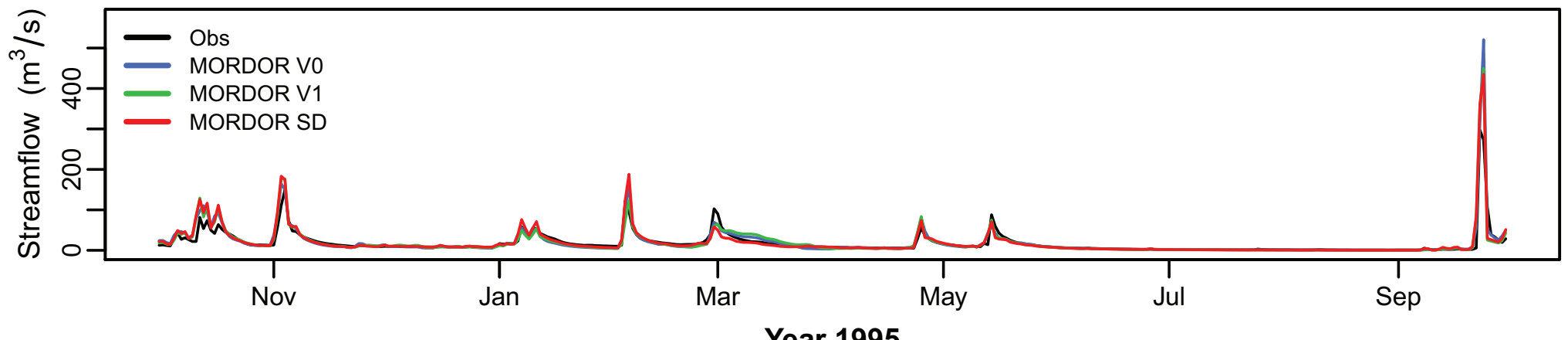

Year 1995

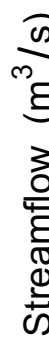

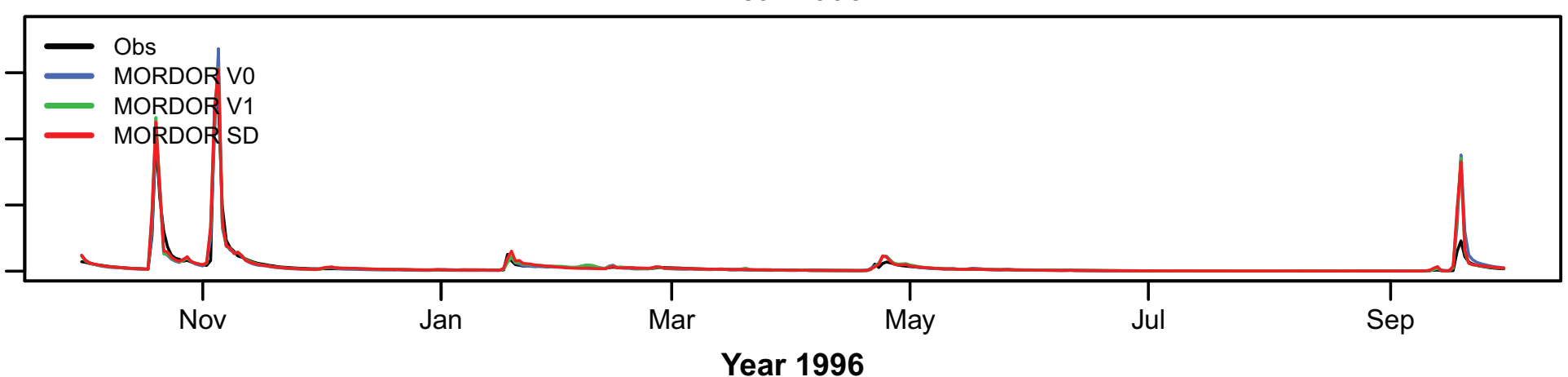

c)

- Obs

Year 1996

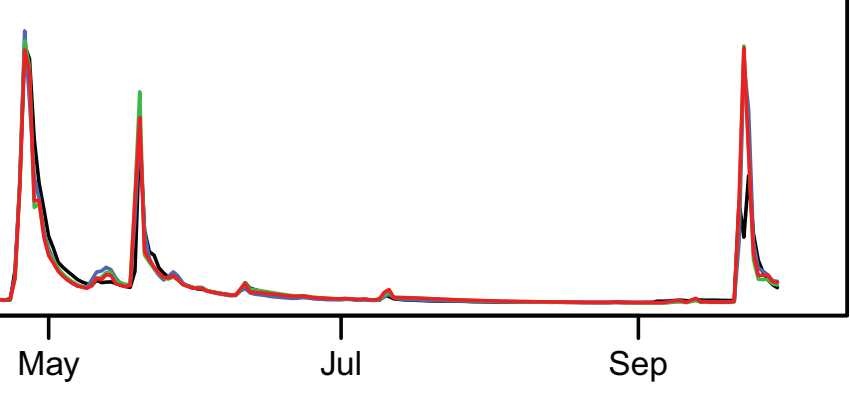


Chassezac@SteMarguerite

Year 2005

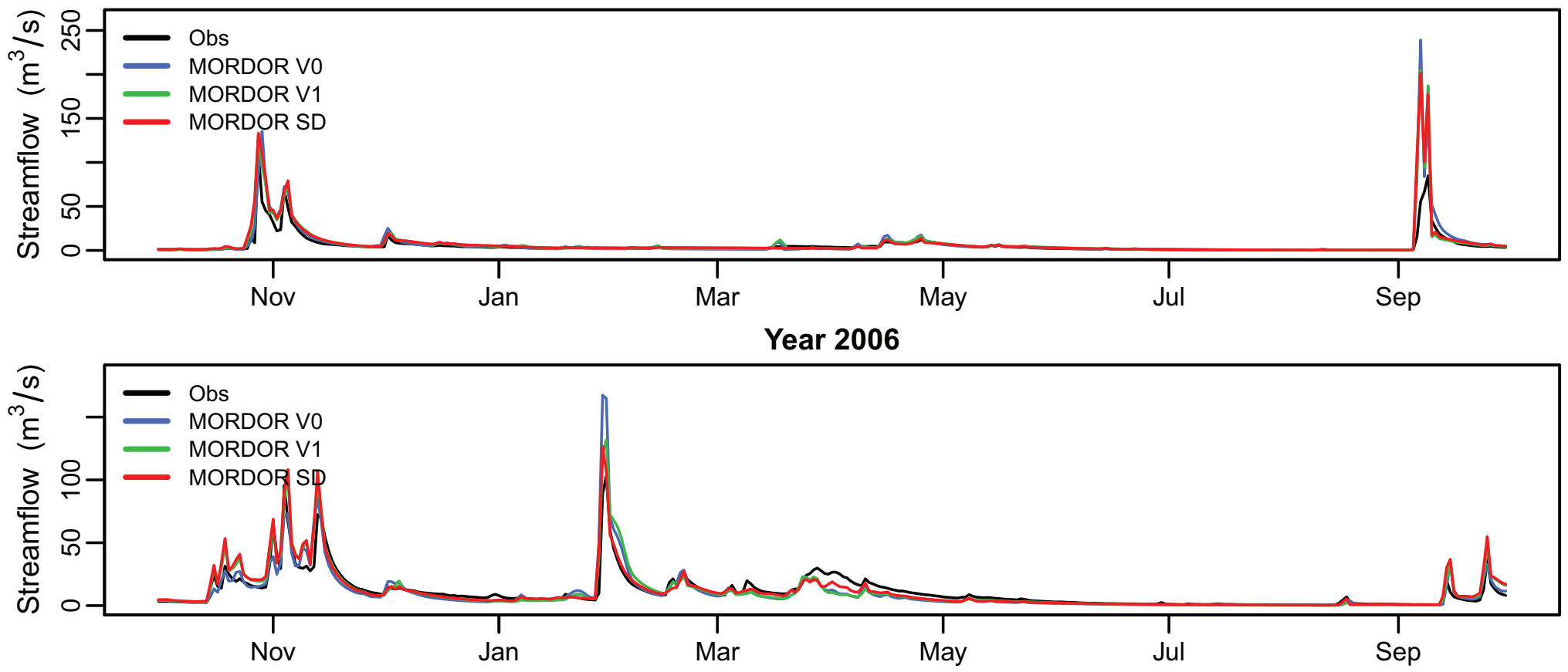




\section{Creuse@Age}

Year 1961
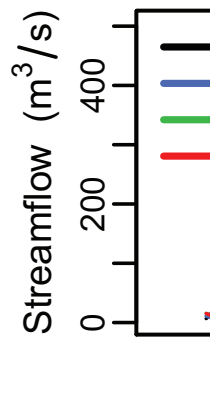

Obs

MORDOR V0

MORDOR V1

MORDOR SD

a. M
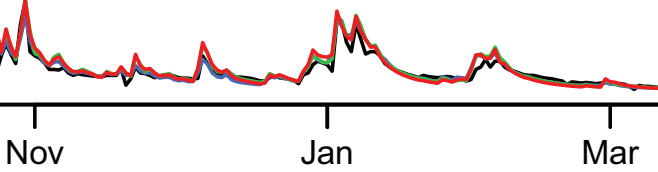

May
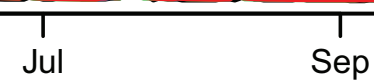

Year 1962

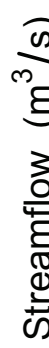

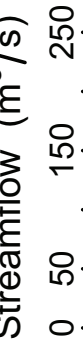
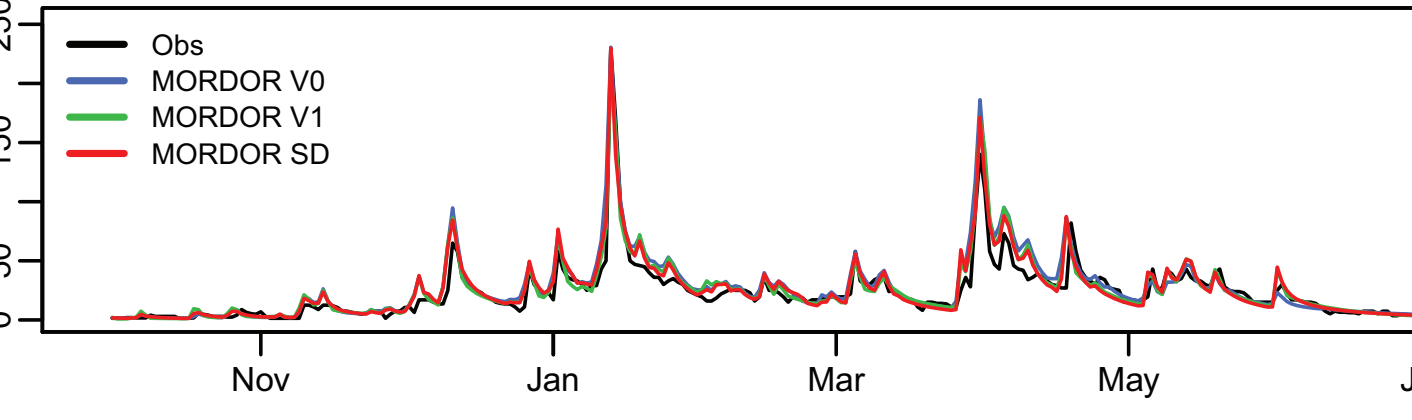

Year 1963
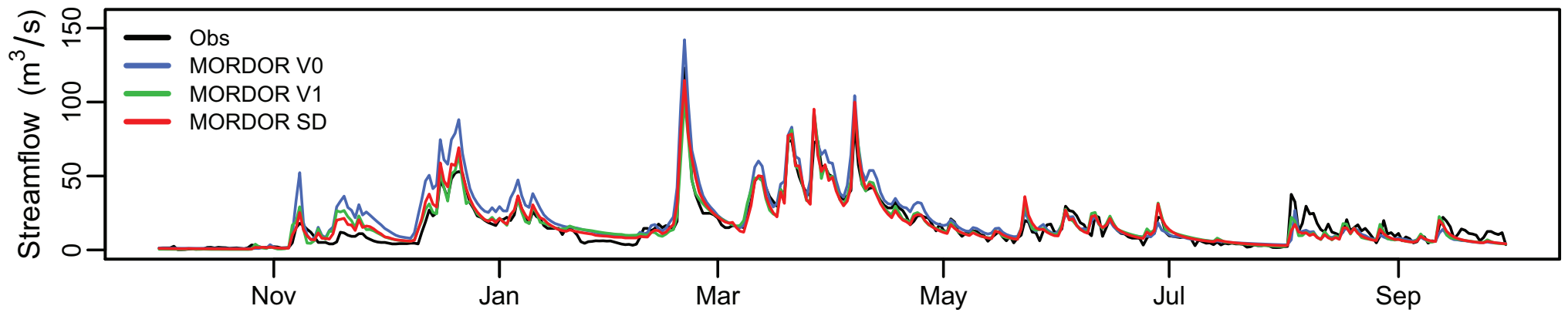

Year 1964
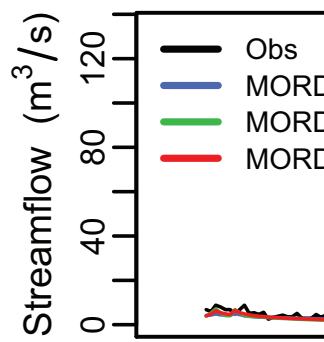

- MORDOR V0

$\longrightarrow$ MORDOR V1

$\infty-$ MORDOR SD

우

○

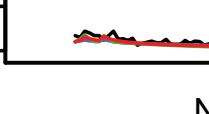

Mmade

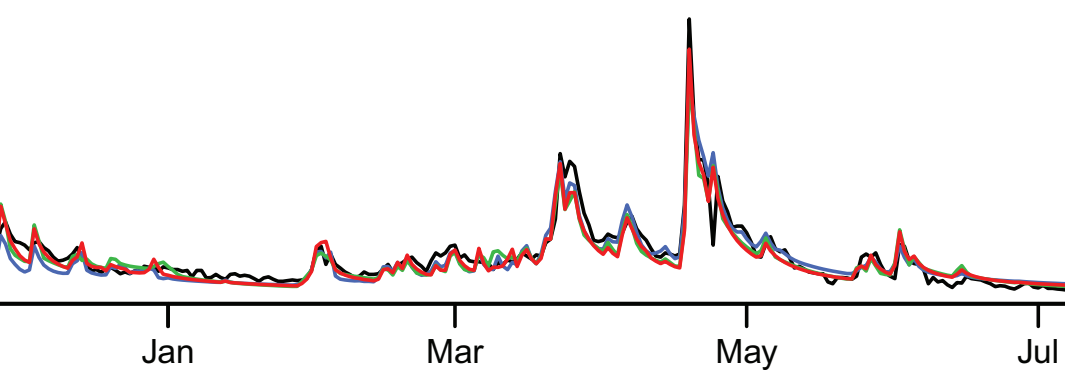

Year 1965
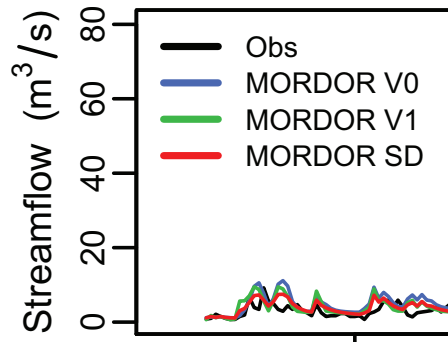

으 $\begin{aligned} & \text { MORDOR V0 } \\ & \text { MORDOR V1 }\end{aligned}$

- MORDOR SD

。

and

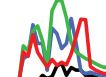
and
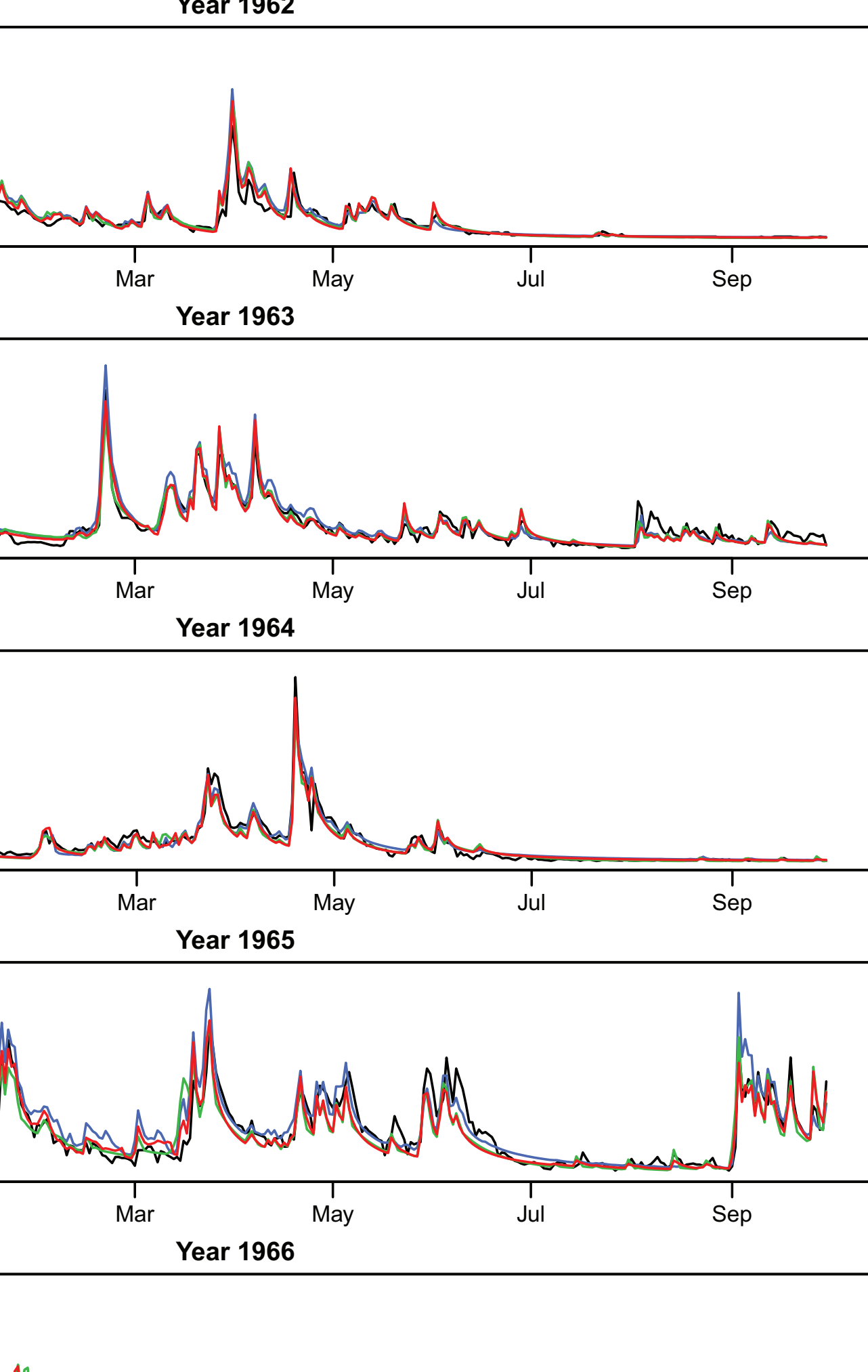

Year 1966
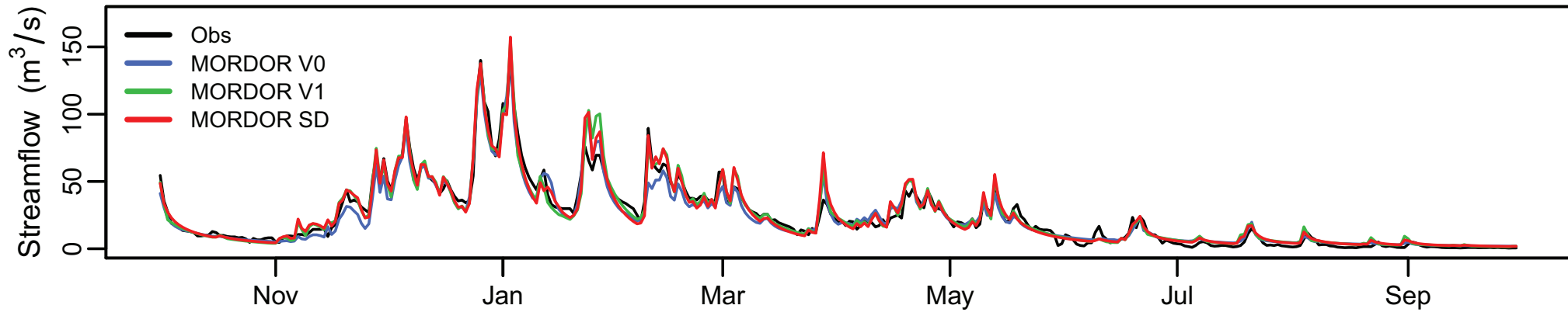


\section{Creuse@Age}

Year 1979
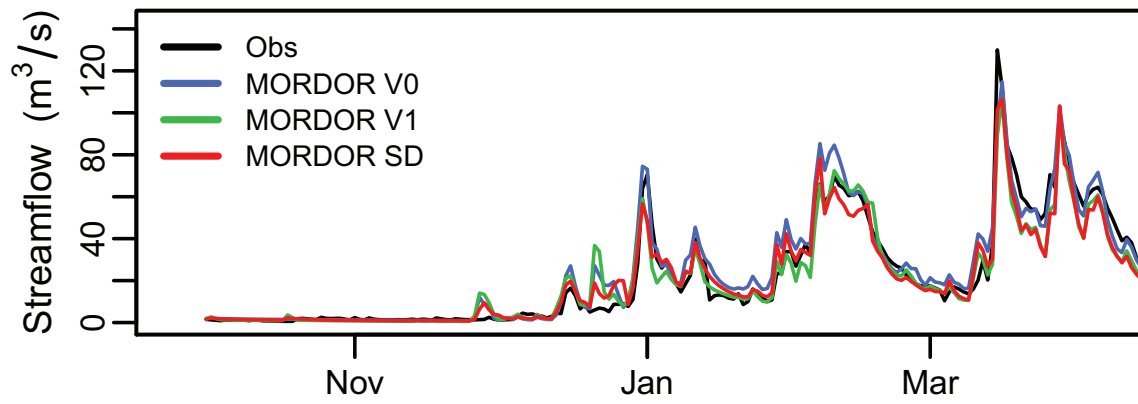

\section{Year 1980}
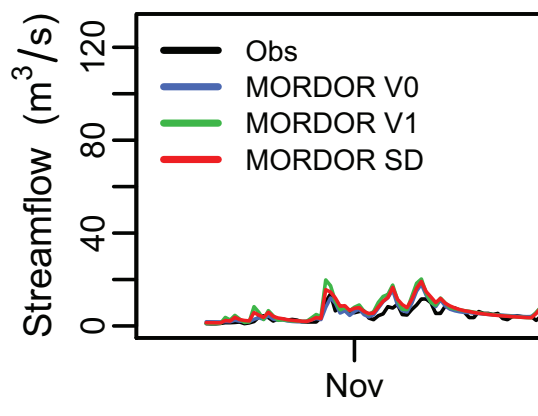

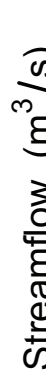

- Obs

- MORDOR V1

o- MORDOR SD

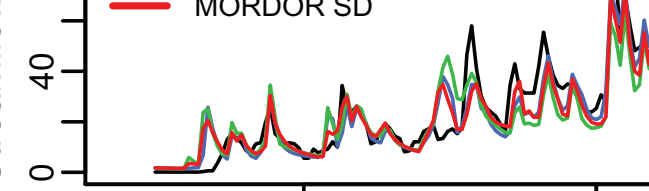

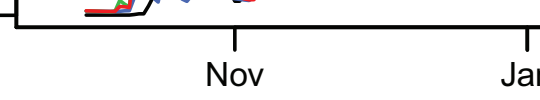

Jan

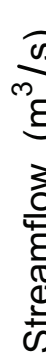

क
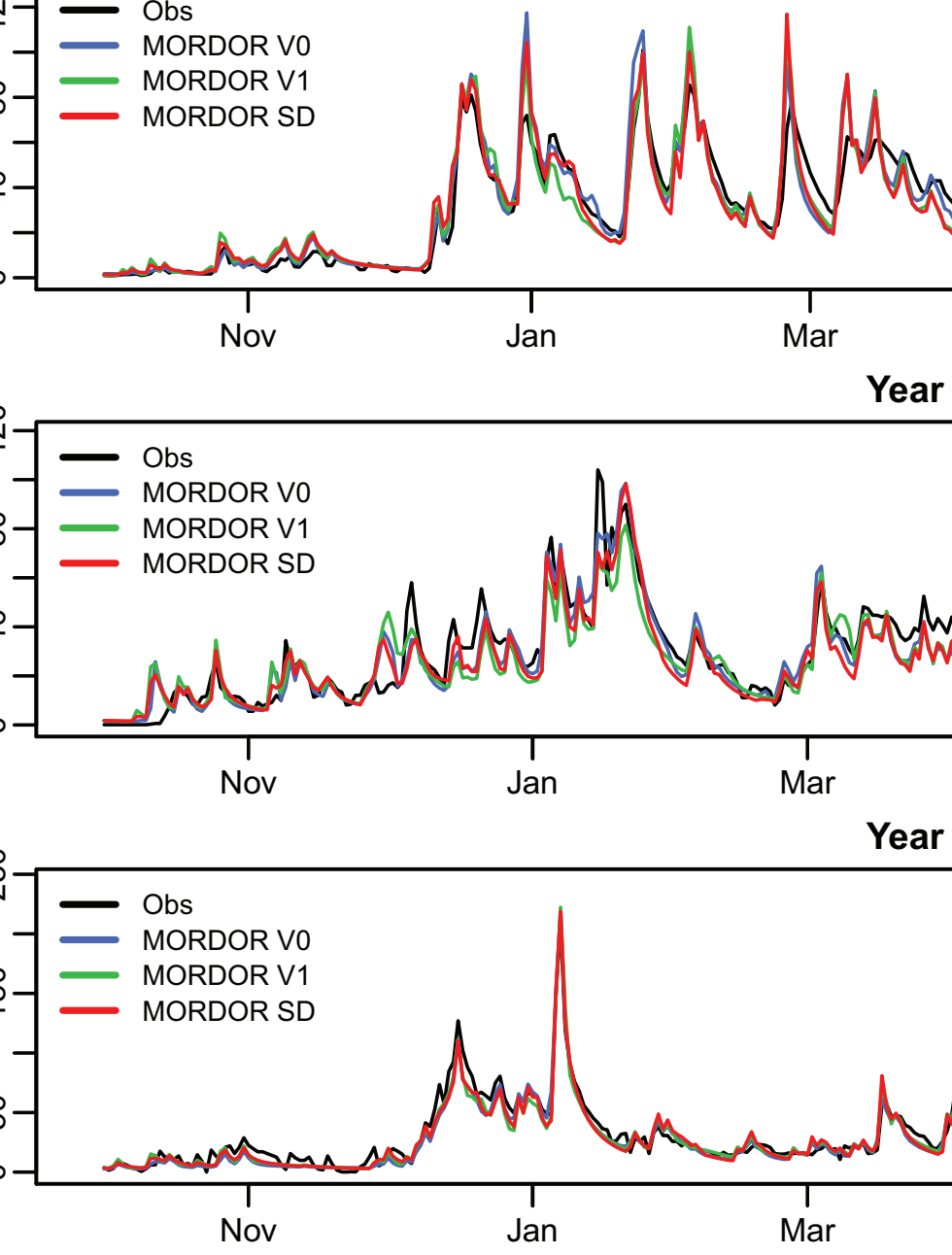

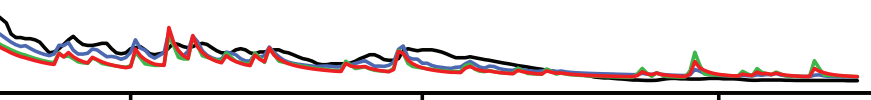

Year 1981

May Jul

Sep

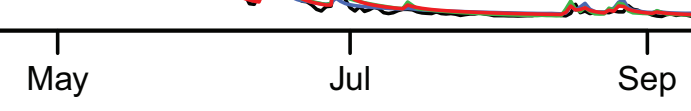

Sep 


\section{Creuse@Age}

Year 1991

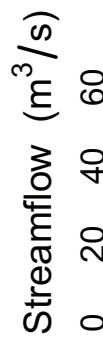

Obs
- MORDOR V0
MORDOR V1

ㅇ- MORDQR SD

Year 1992

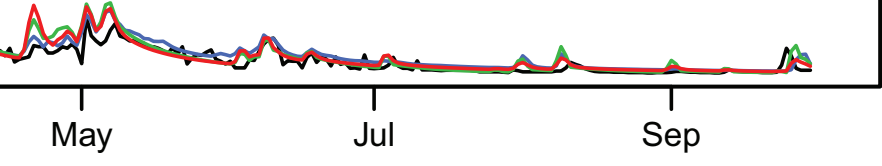

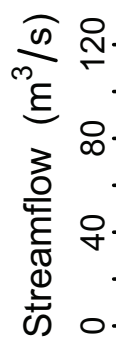

- Obs

- MORDOR V0

MORDOR V1

MORDOR SD

ㅇ-

ancosta

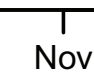

Nov

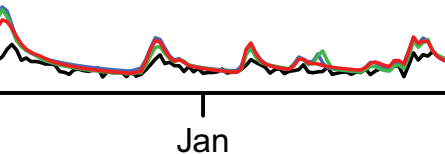

Year 1993

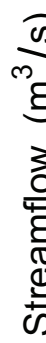

- Obs

- MORDOR V0

-1
$\infty-1$
$D_{-1}$

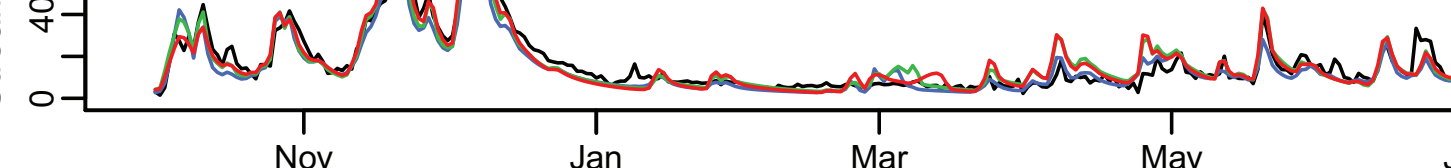

Jan Mar

May

Year 1994

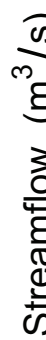
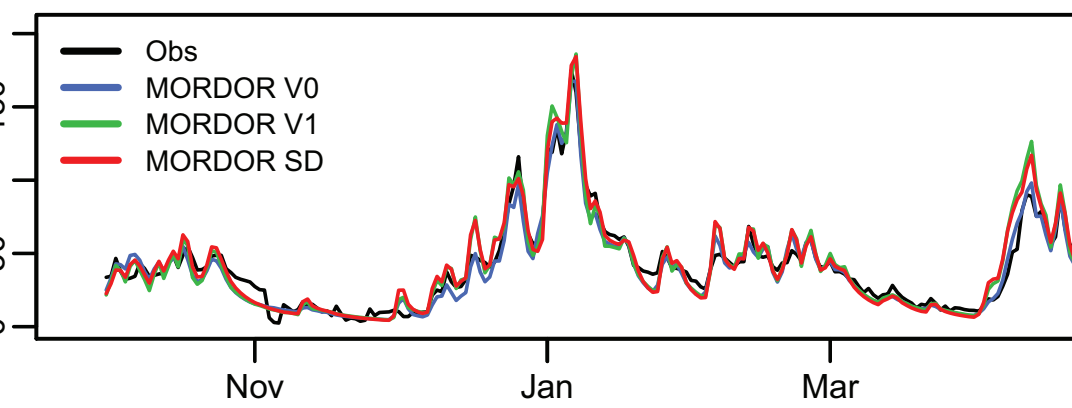

y.am

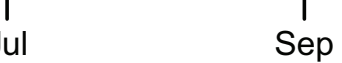

Sep

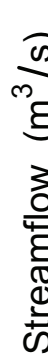

Year 1995
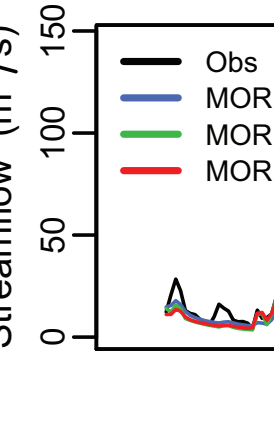

$-\begin{aligned} & - \text { MORDOR V0 } \\ & \text { MORDOR V1 }\end{aligned}$

$\longrightarrow$ MORDOR SD

M M Monk
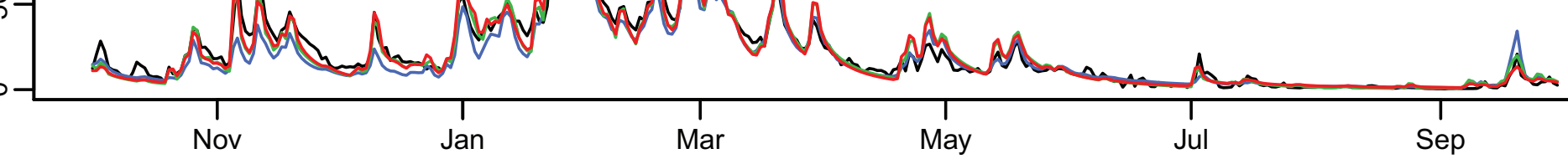

Year 1996
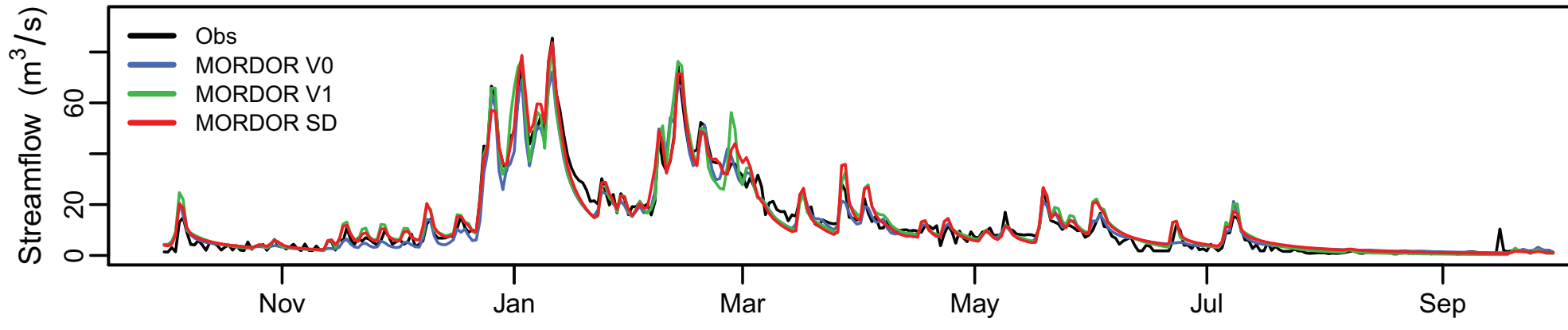
Dordogne@Bort

Year 1980

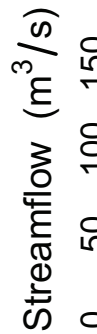

- obs

- MORDOR V0

MORDOR V1

- MORDOR SD

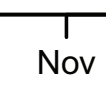

Jan

Mar

Year 1981

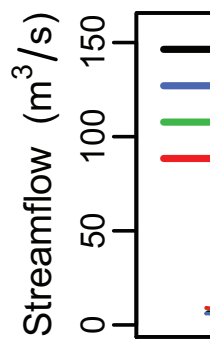

Obs

- MORDOR V0

- MORDOR V1

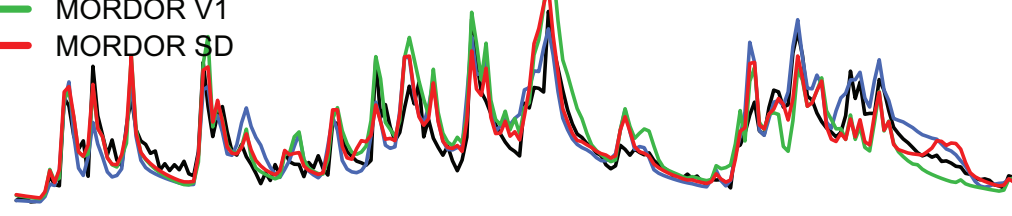
Nov Jan

Mar

Year 1982

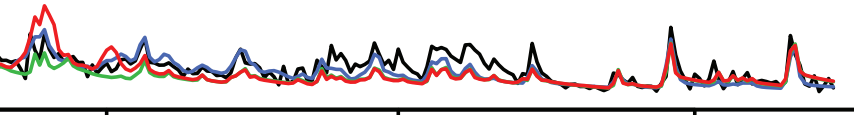
May

Sep

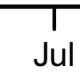

$\frac{0}{3}$

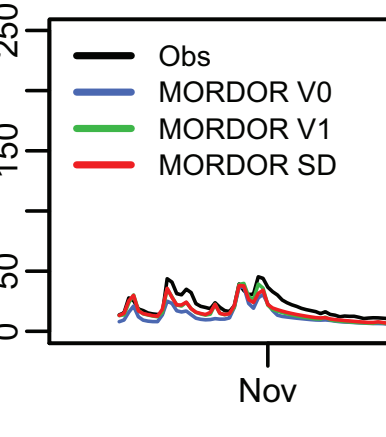

-
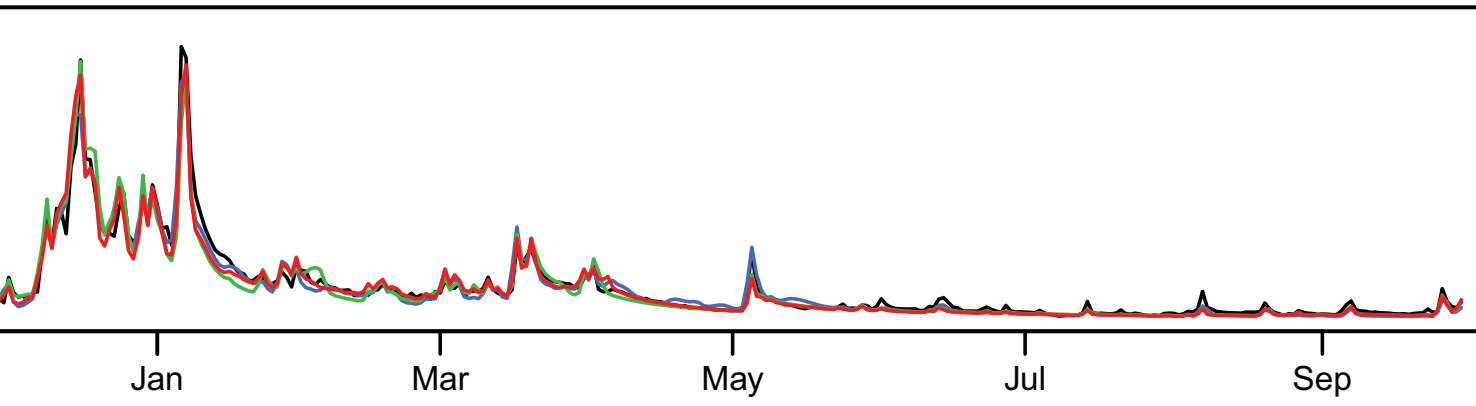

Year 1983

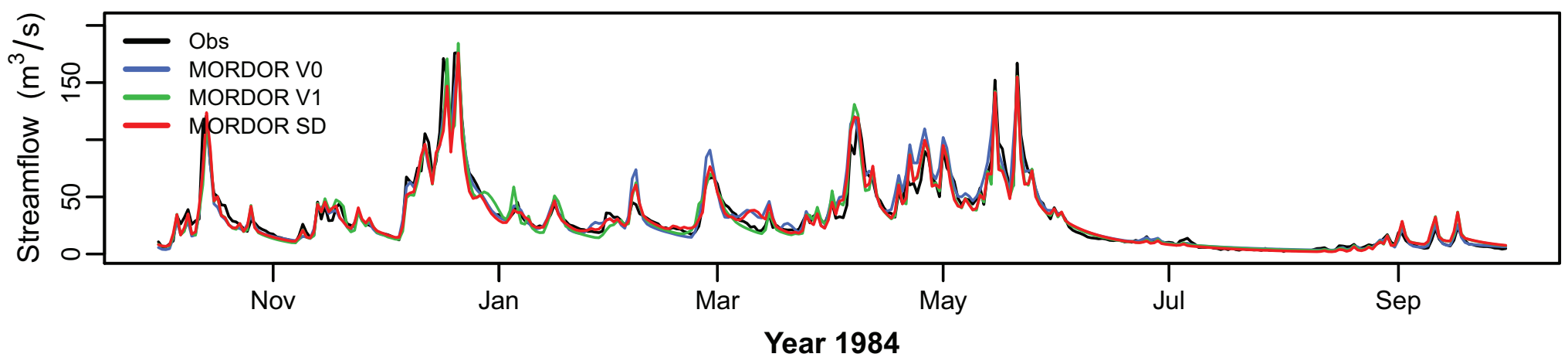

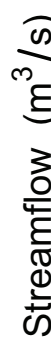
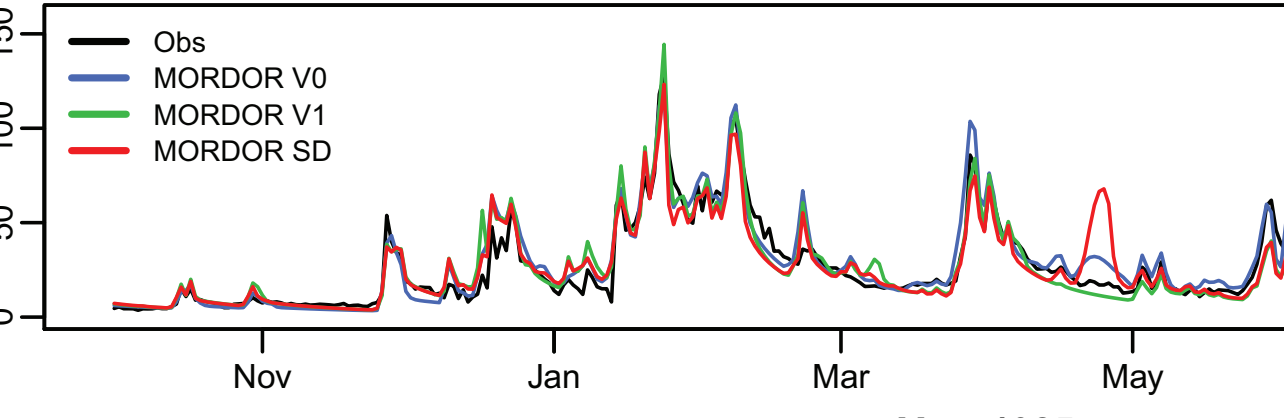

Year 1985
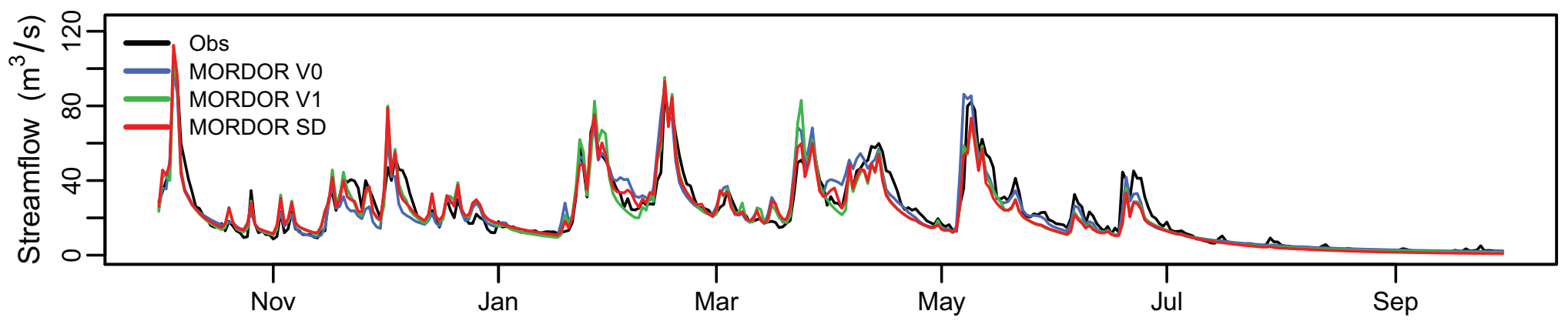
Doubs@Brenet

Year 1987

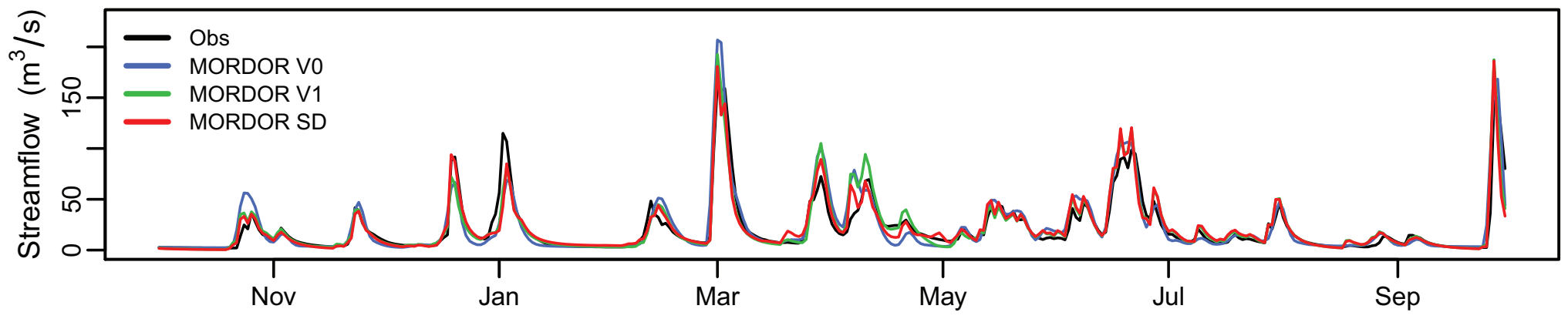

Year 1988
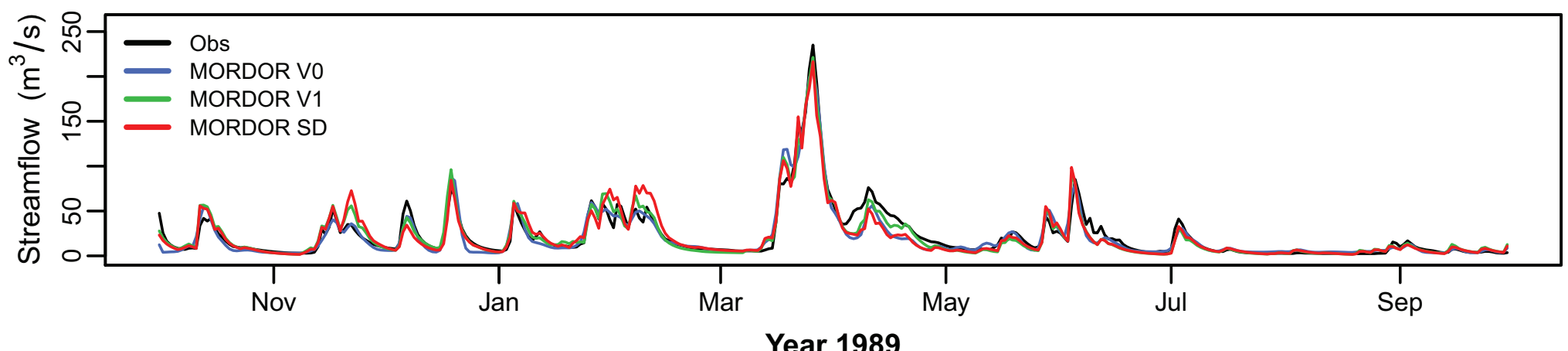

Year 1989

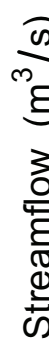

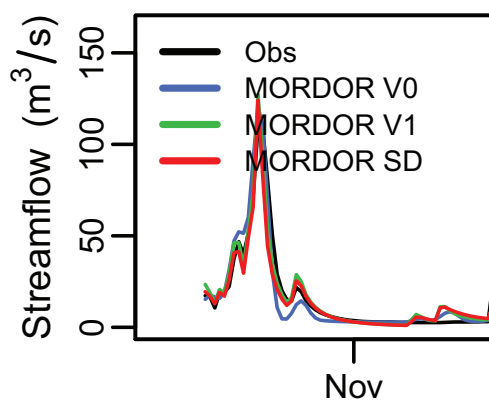

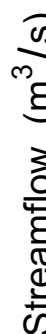

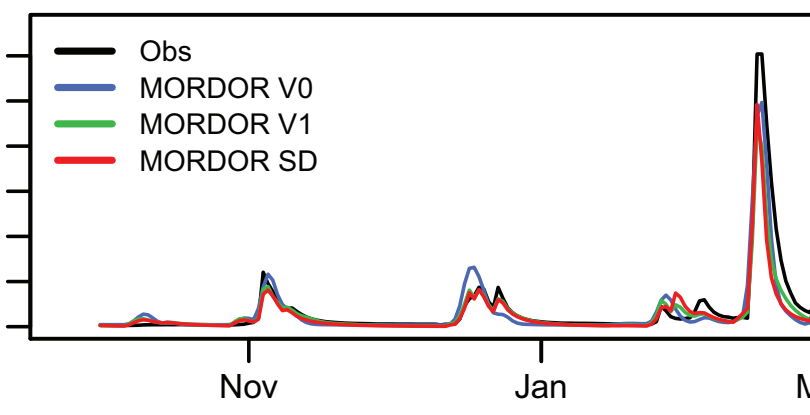

Year 1990

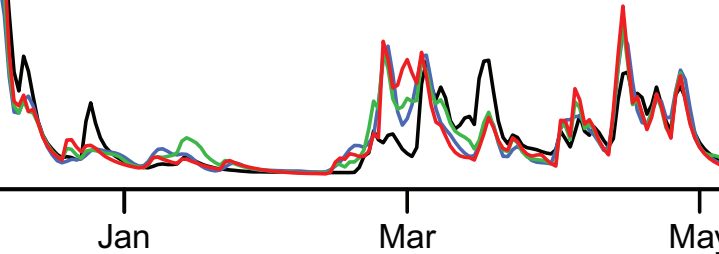

Year 1991
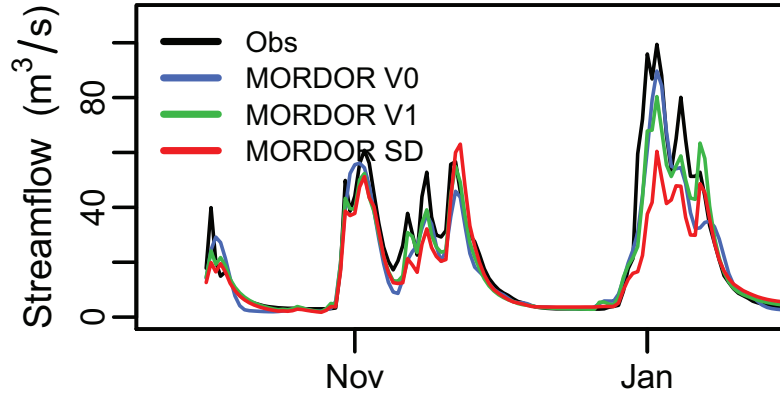

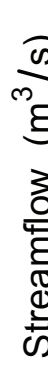
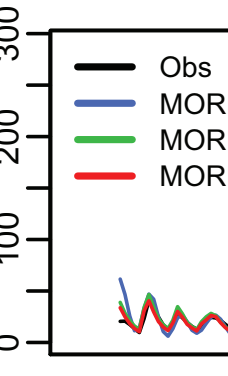

- MORDOR VO

- MORDOR V1

(MDOR

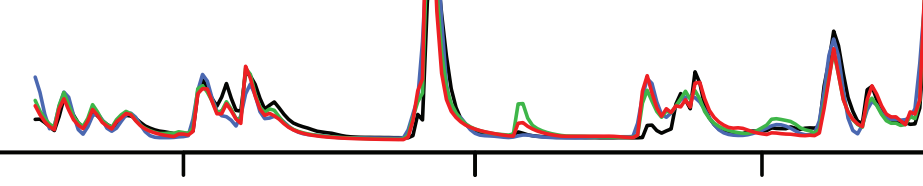


Doubs@Brenet

Year 1999

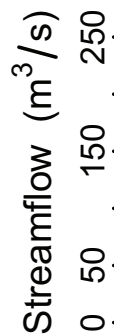

Obs

MORDOR V0

MORDOR V1

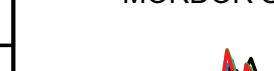

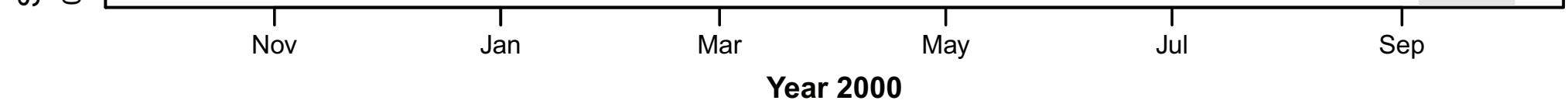

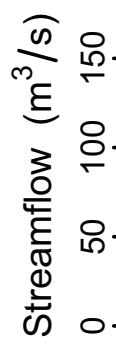
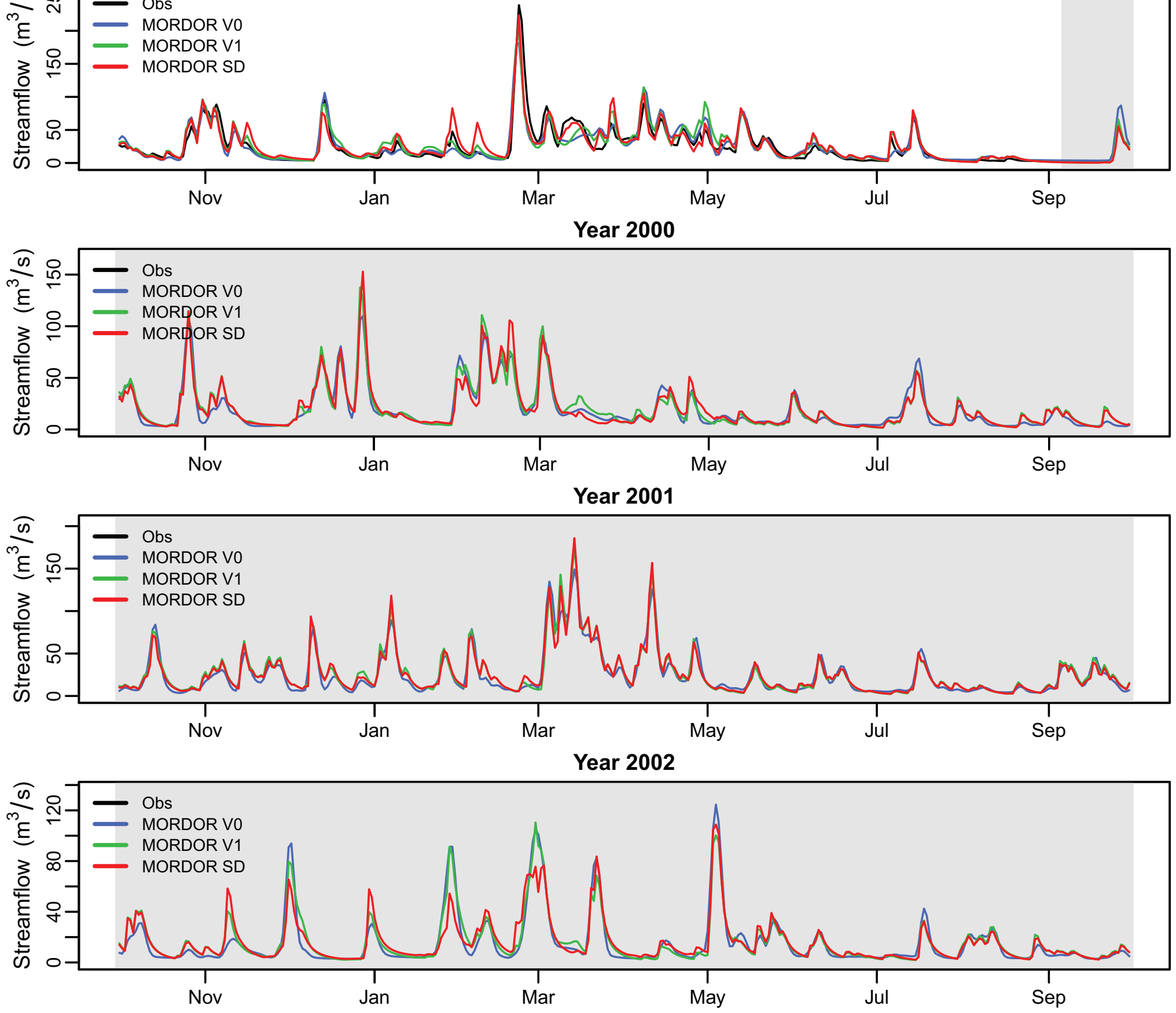

0
m
है
3
0
0
$\frac{1}{0}$
0
0

Year 2003

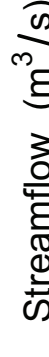

है
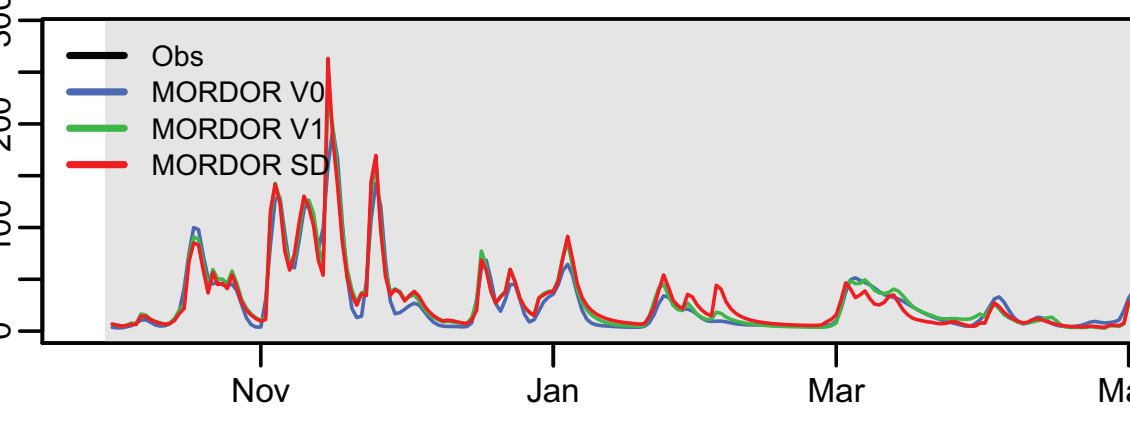

Year 2004
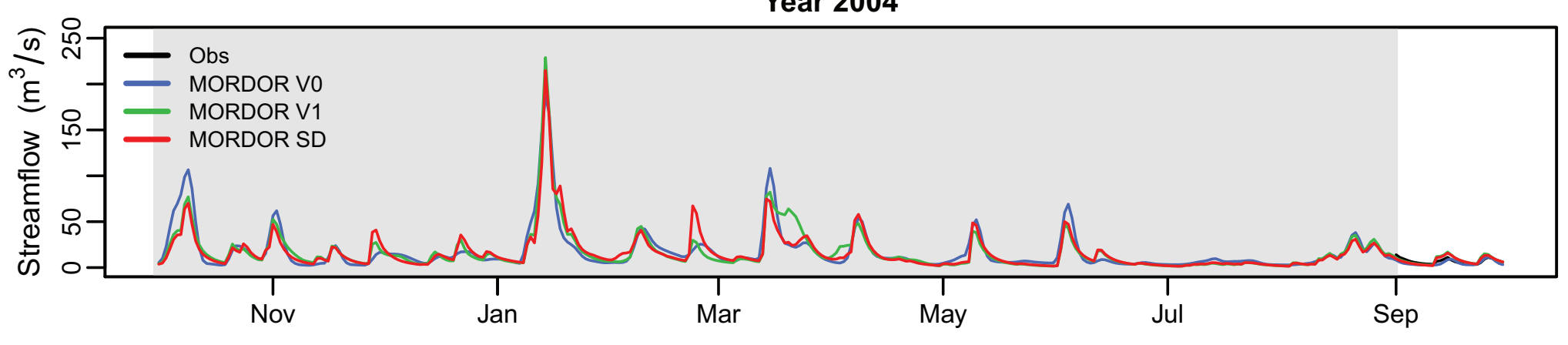


\section{Doubs@Neublans}

\section{Year 1974}

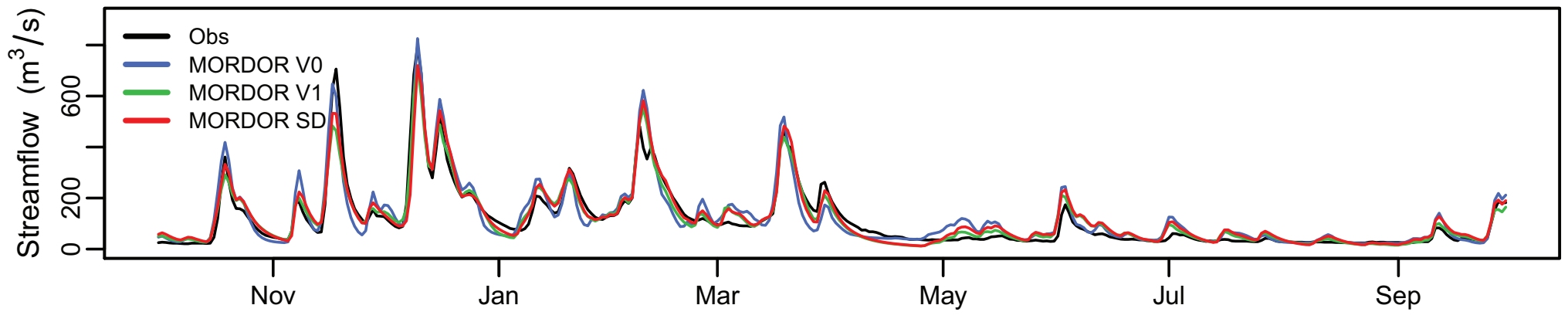

Year 1975
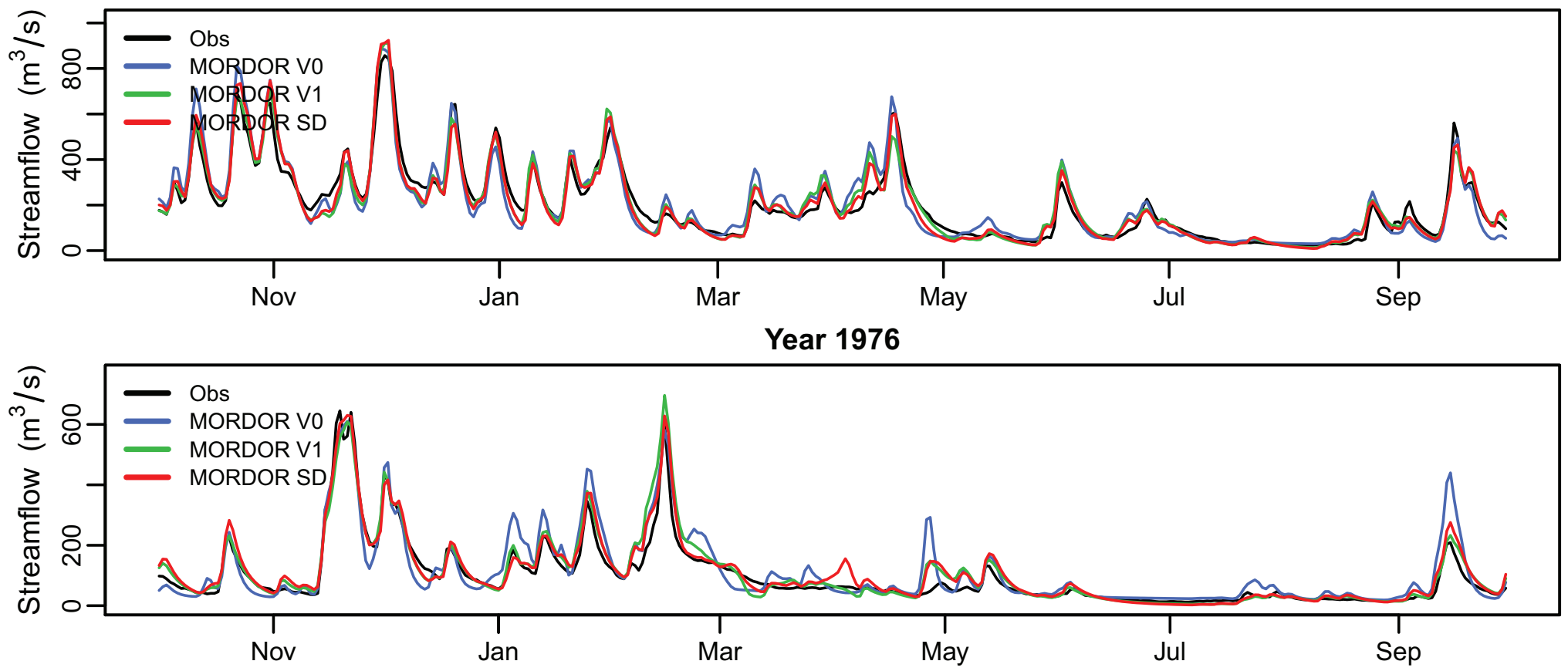

$\frac{1}{3}$

Year 1977

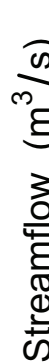

है

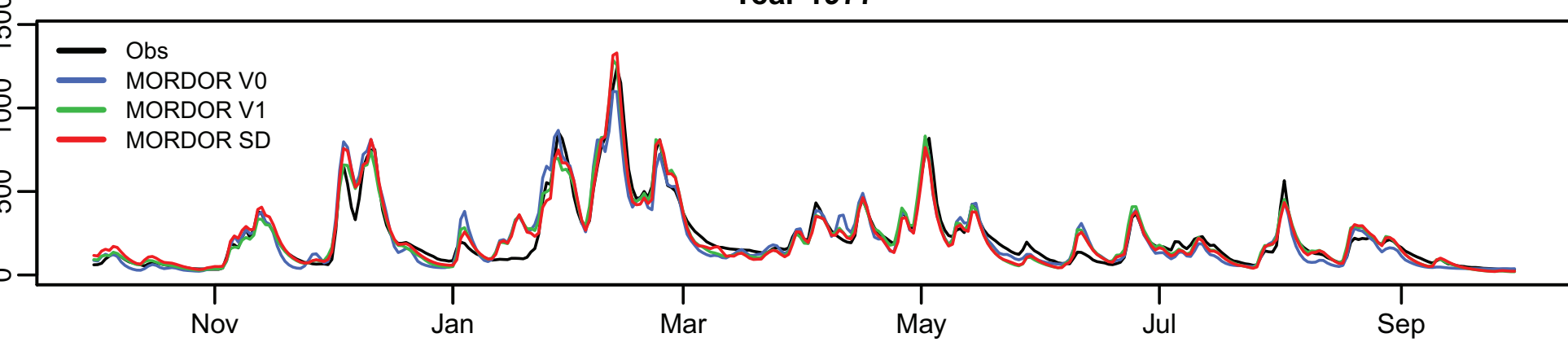

Year 1978

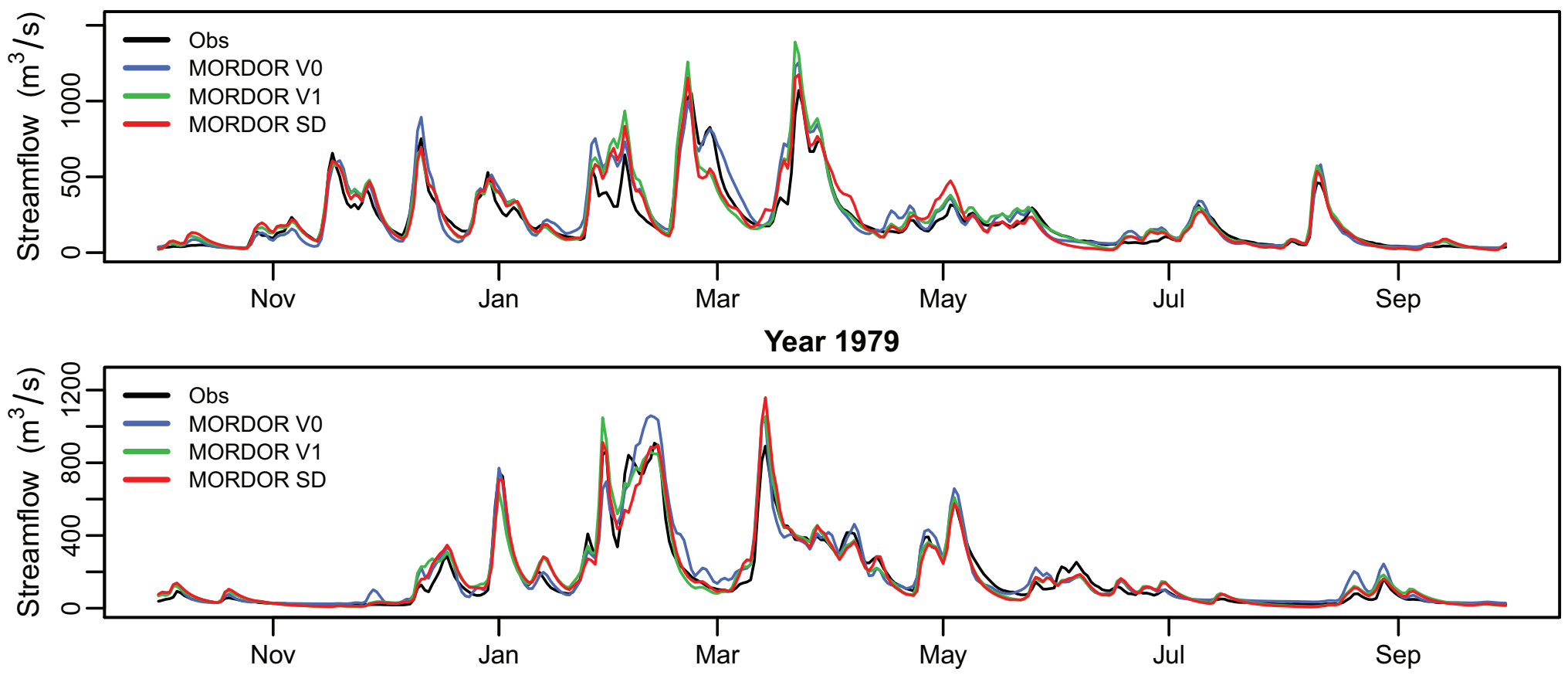




\section{Doubs@Neublans}

Year 1998
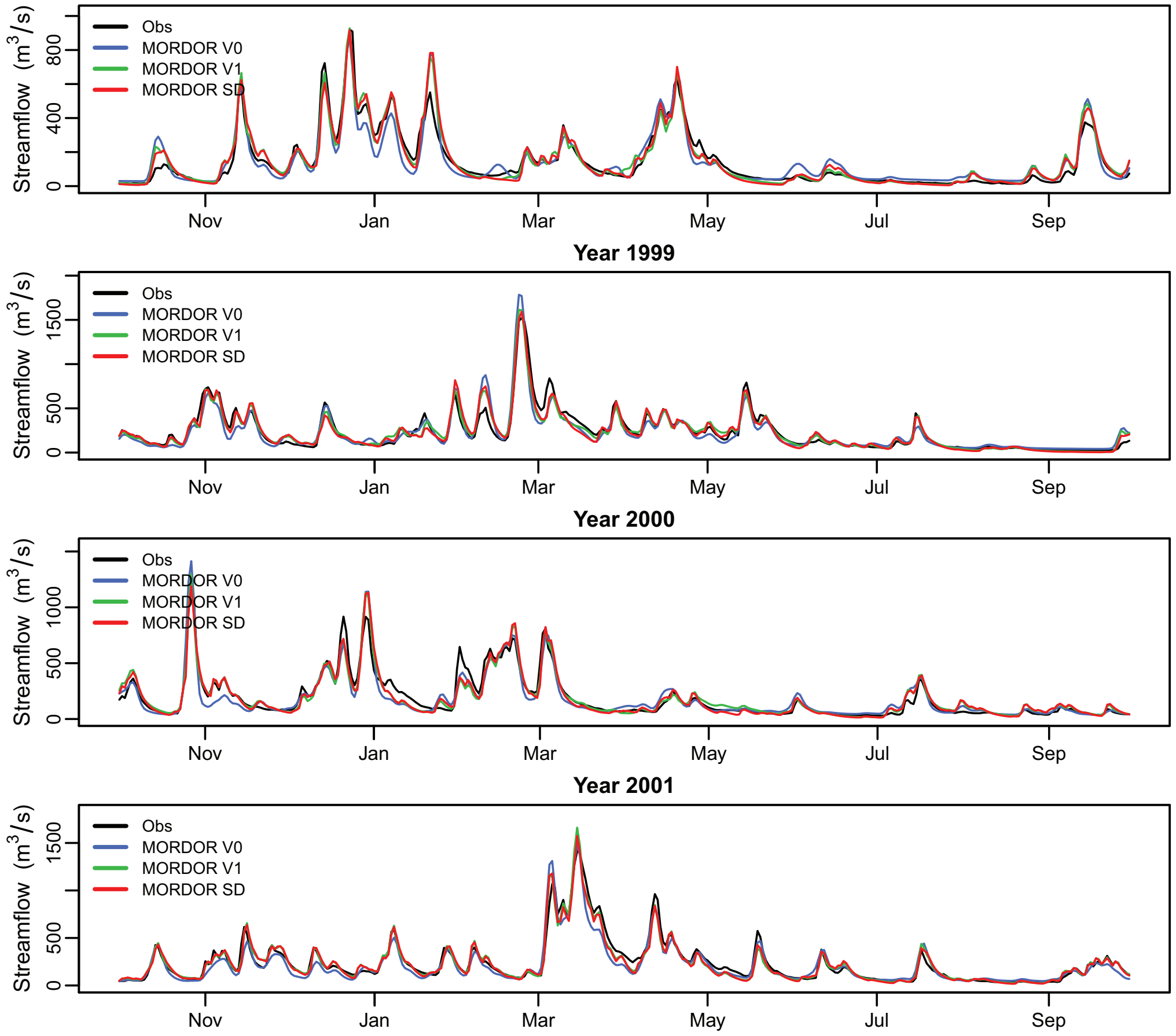


\section{Drac@Sautet}

\section{Year 1976}

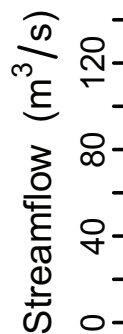

Obs

MORDOR V0

$\longrightarrow$ MORDOR V1

$\infty-\longrightarrow$ MORDOR SD

.

be Nov

nh

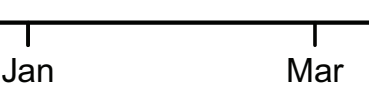

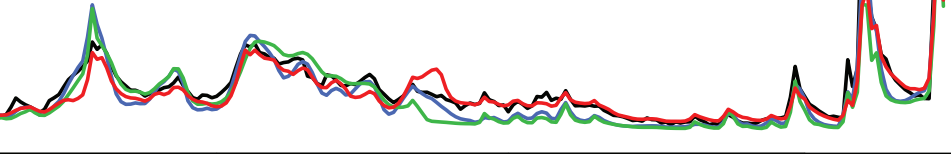

Year 1977

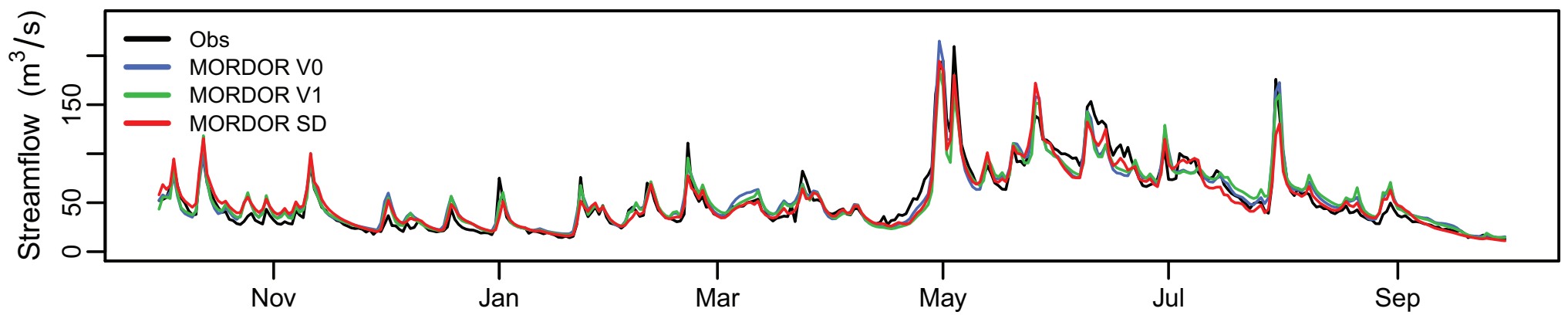

Year 1978

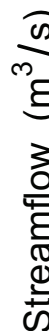

$-{ }_{-}$

Obs

MORD V

MORDOR SD

the

Nov

Jan

Year 1979

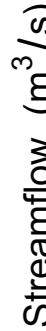

$\longrightarrow$ Obs

- MORDOR V0

으- MORDOR SD

.

.

No

Nov

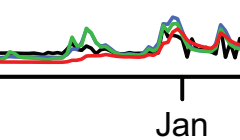

Jan

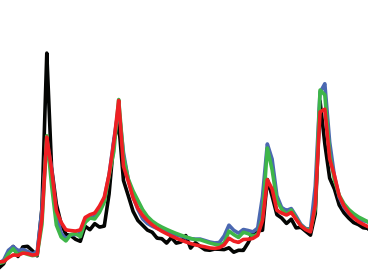

nomb

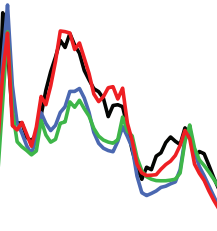

Mar May

Year 1980

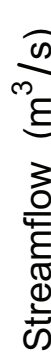

西

- Obs

- M MRDOR vo

- MORDOR SD

.

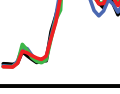

nana a n

Jan

Mar

Year 1981

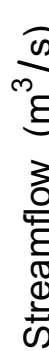

年

- obs

- MORDOR V0

- MORDOR V1

- MQRDOR SD

M Mana 


\section{Durance@Clapiere}

Year 1995
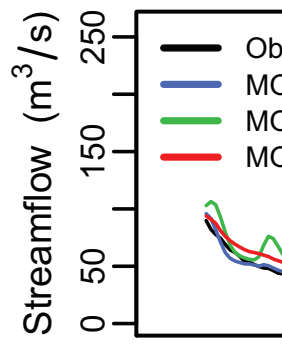

\section{Obs}

ORDOR V1

ORDORjSD

ad

Nov

2
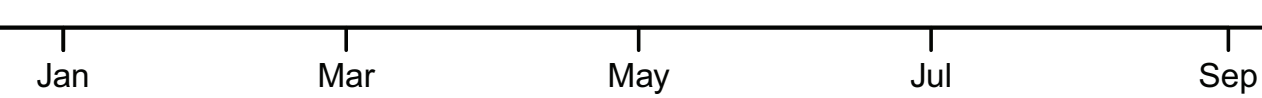

Year 1996

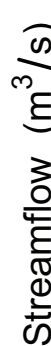

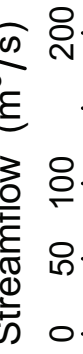

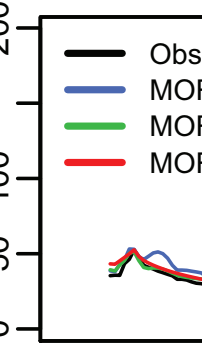

\section{bs}

RDOR V0

ORDOR SD
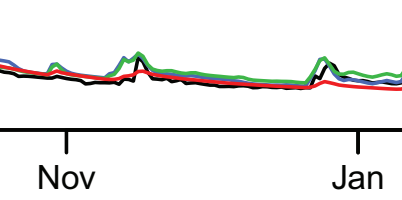

Mar

Year 1997

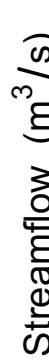

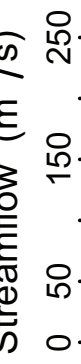

-

- Obs

- MORDOR VO

- MORDOR V1

- MORDOR SD

A

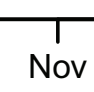

Nov

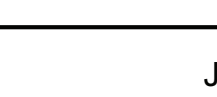

Jan

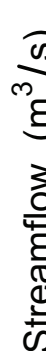
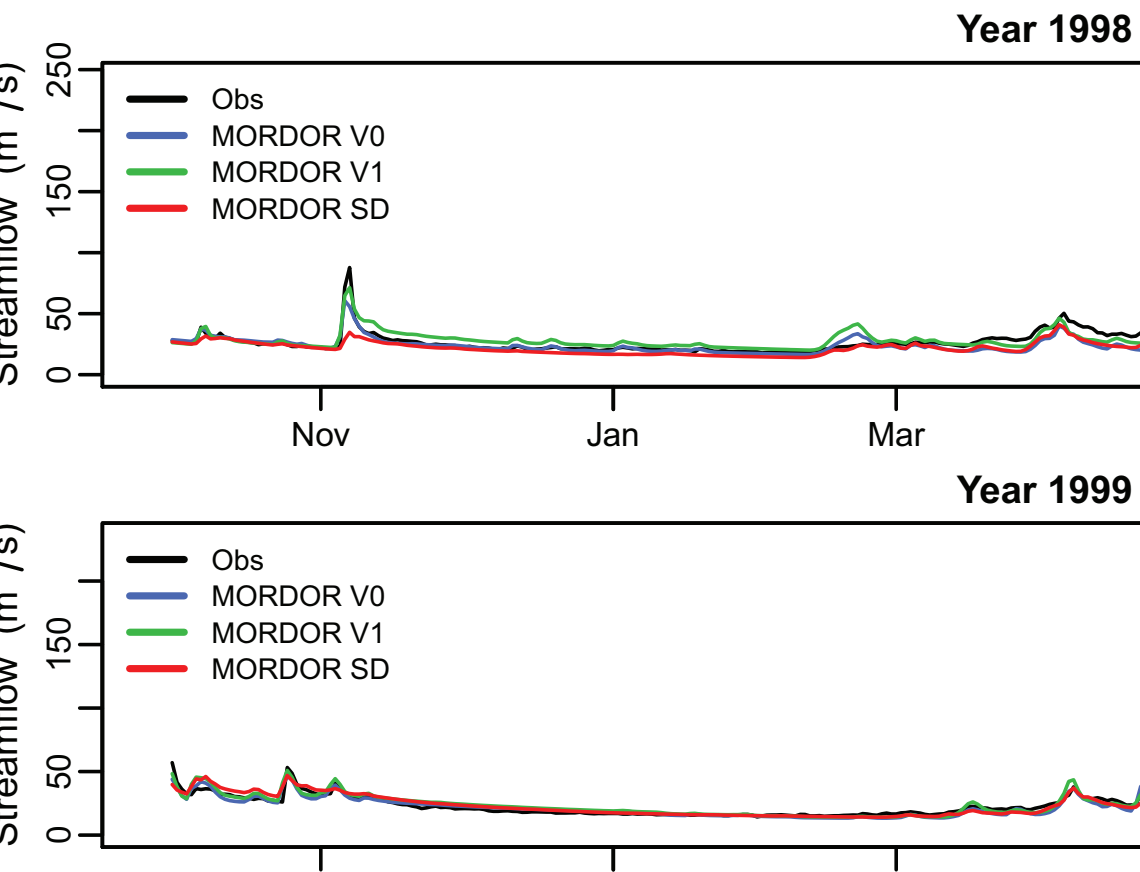

Year 1998
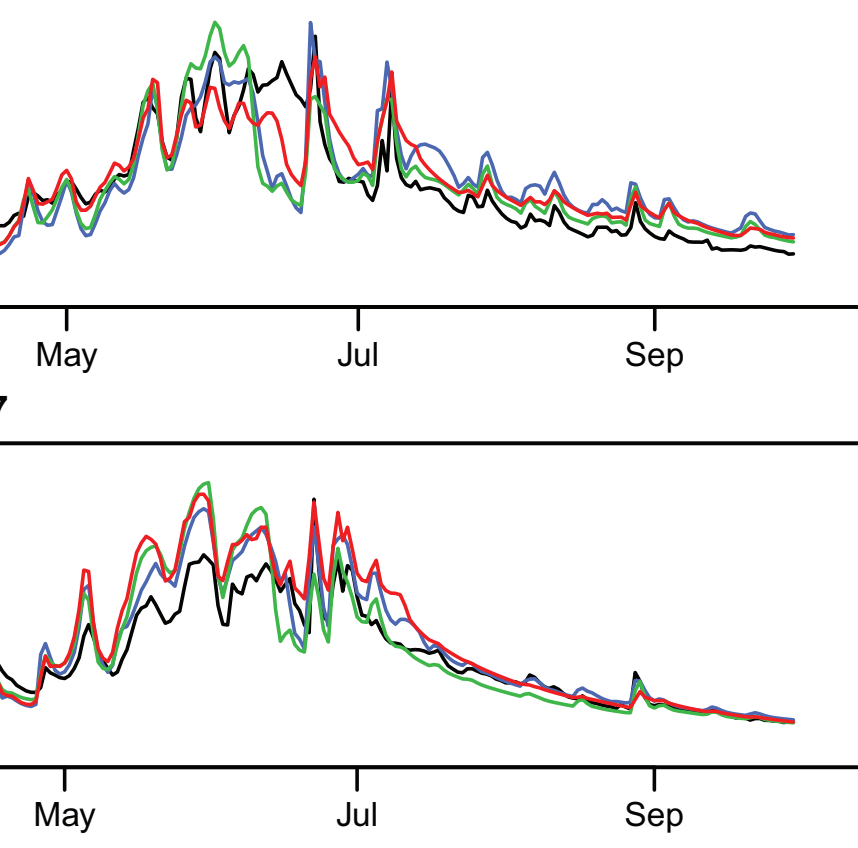


\section{Durance@Clapiere}

Year 2001
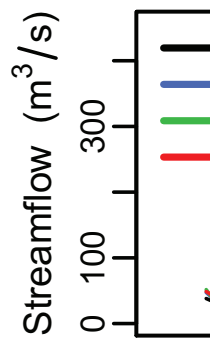

$$
\text { Obs }
$$

MORDOR V0

MORDOR V1

MPRDOR SD

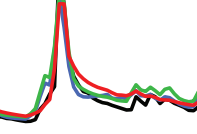

Nov
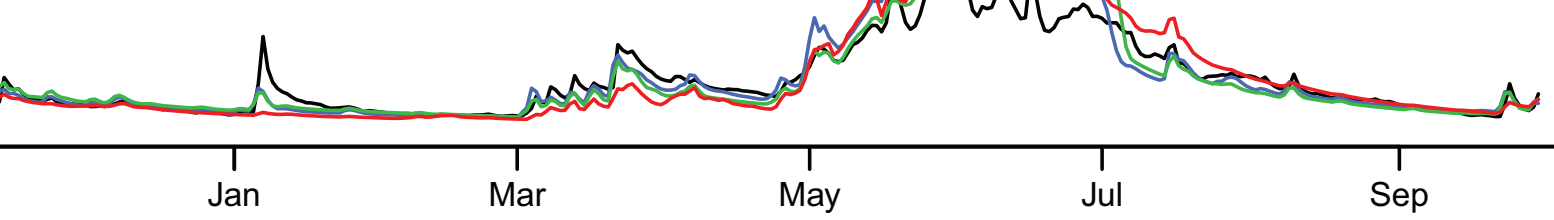

Year 2002
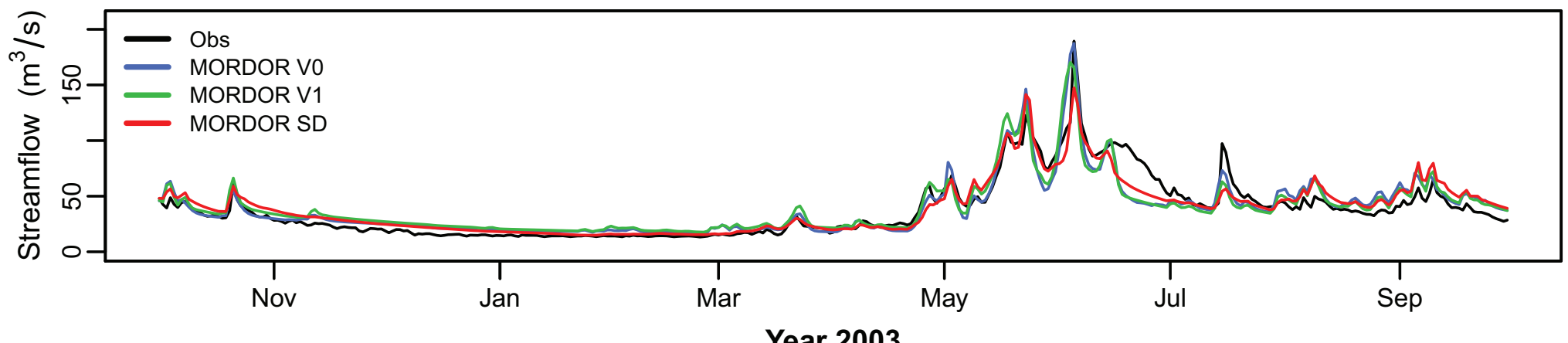

Year 2003

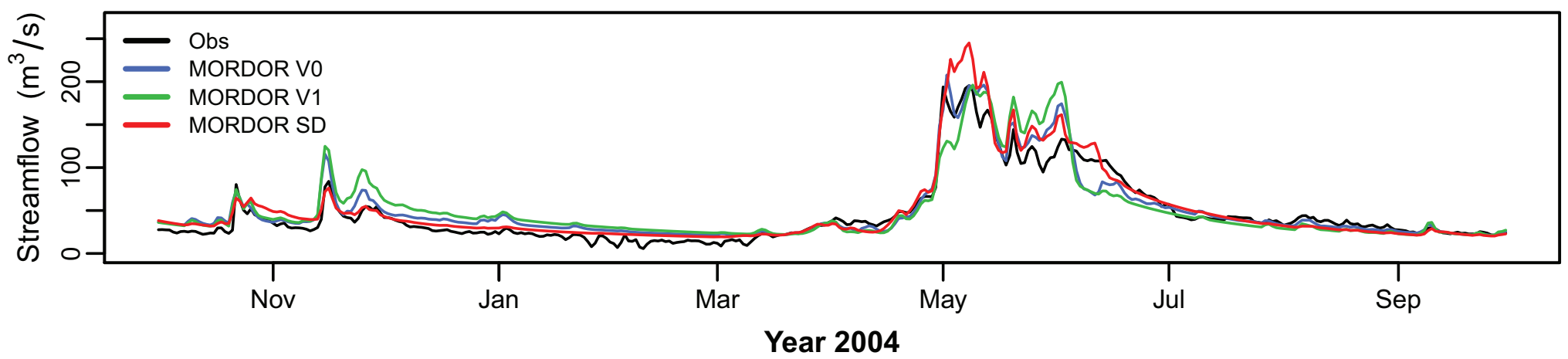

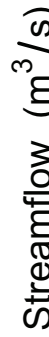

- Obs

MORDOR V0

MORDOR V 1

— MORDOR SD

$-$

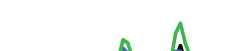




\section{Gage@Gagell}

Year 1993

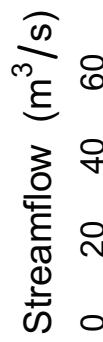

- Obs
MORDOR VO
MORDOR V1

$\longrightarrow$ MORDOR SD
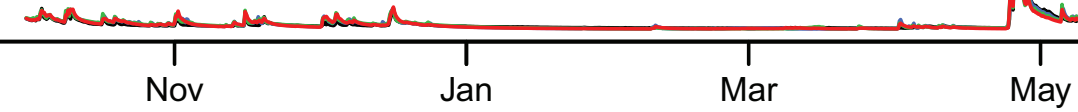

Year 1994
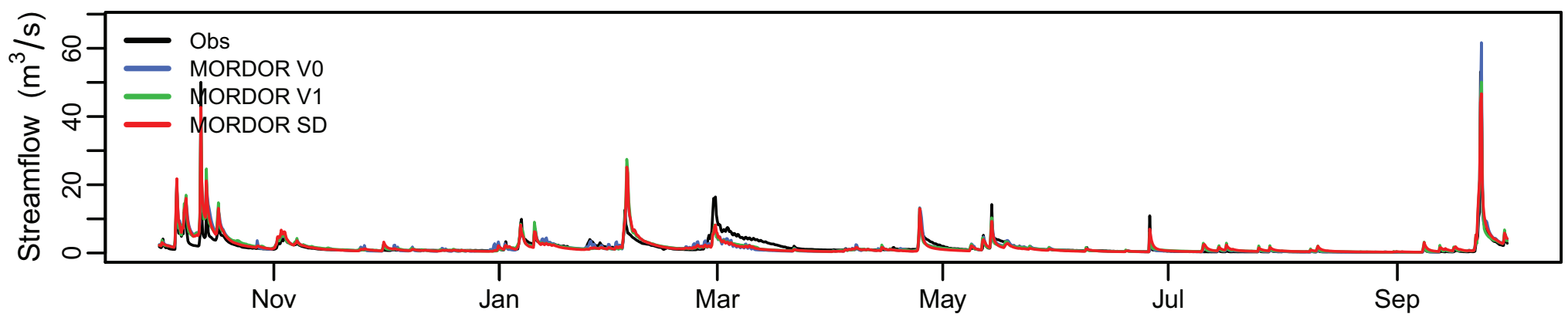

Year 1995

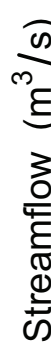

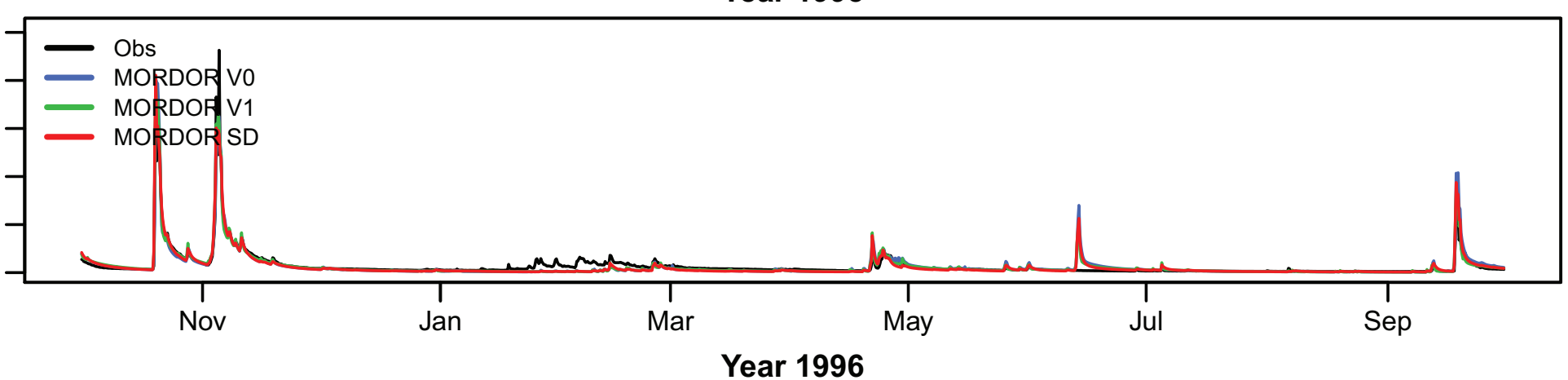

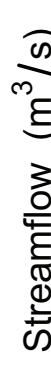

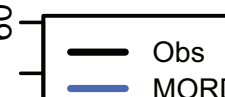

- - MORDOR VO

$\checkmark-\longrightarrow$ MORDOR V1

MORDOR SD

s le culut lla

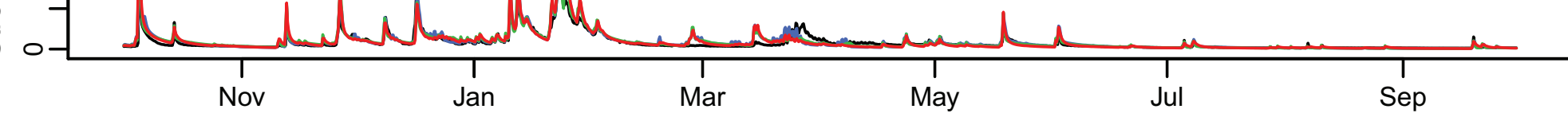

Year 1997

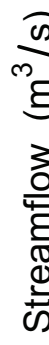

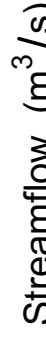

ह

- Obs
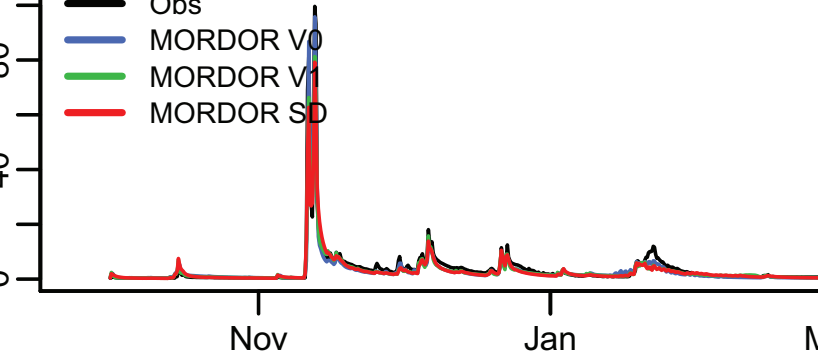

Mar

Year 1998
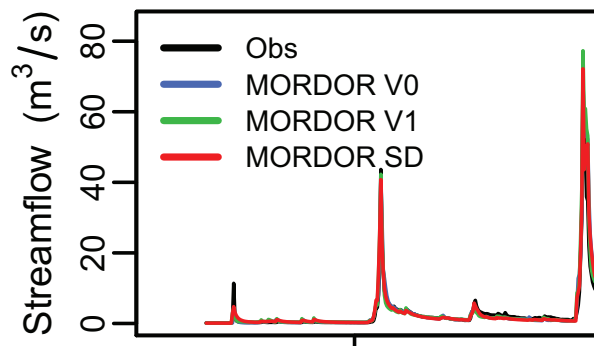
Gardon@Corbes

Year 1999
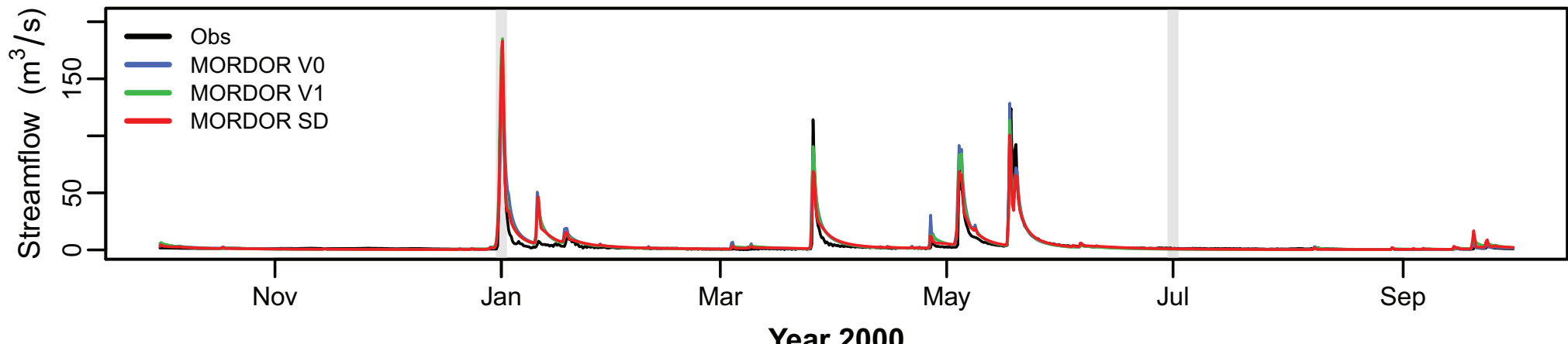

Year 2000

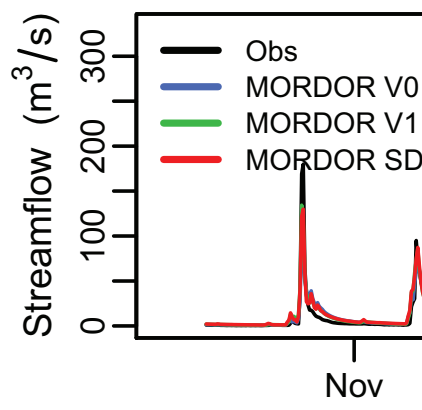

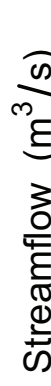

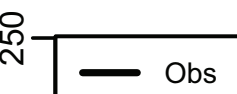

8

MORD

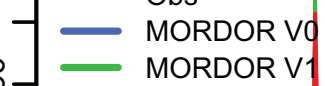

moroors

Nov

Jan

Year 2001

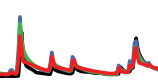

May

1

Sep

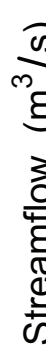

- Obs

- MORDOR V0

MORDOR V1

+- MORDOR SD

ㅇ-

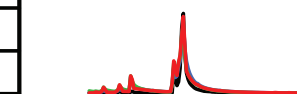

.

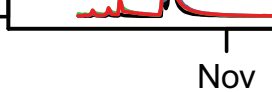

Nov

Jan

Mar

Year 2003

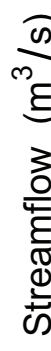

$-\mathrm{Obs}$

- MORDOR V0

- MORDOR V1

-1 MORDOR SD

은

$\circ-$
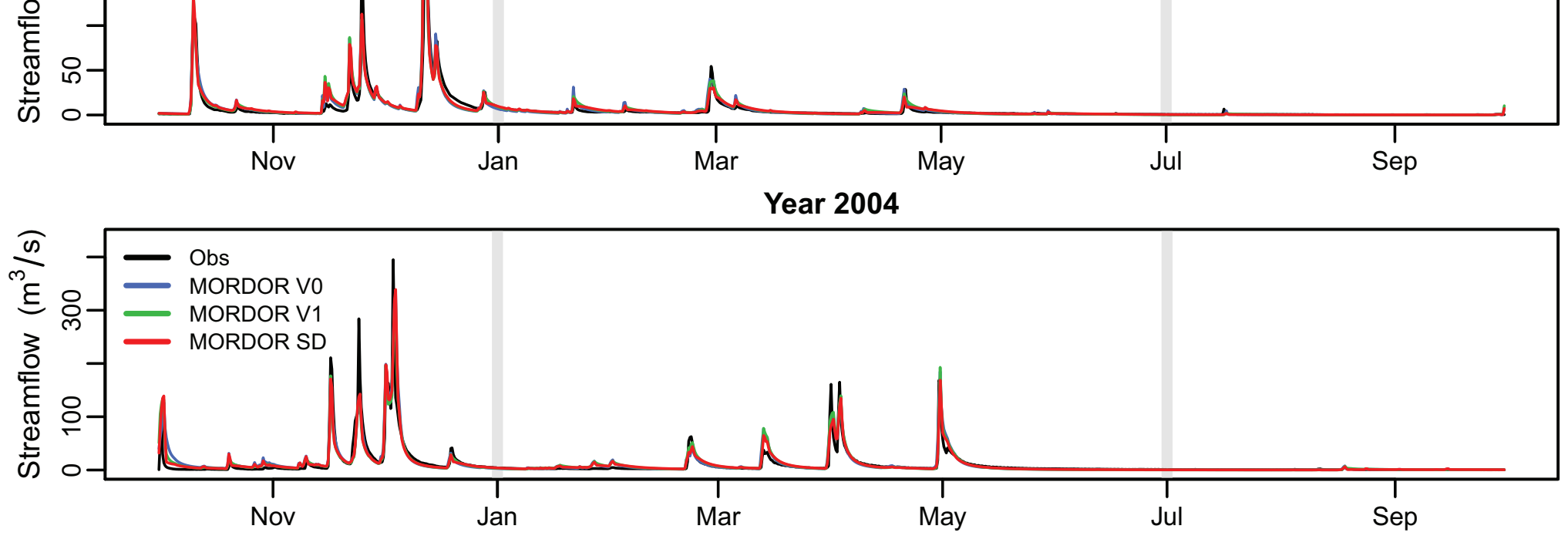
GaveEstaube@Gloriettes

Year 1994

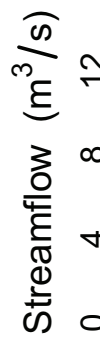

E⿰冫欠

RDOR V0

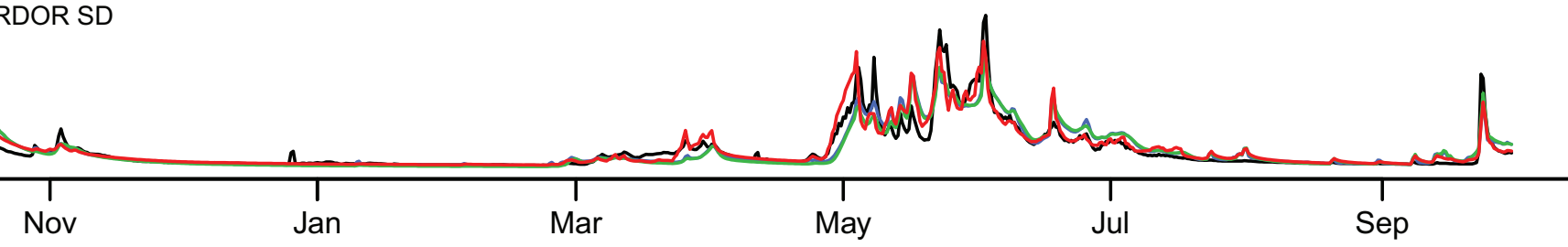

Year 1995

$\frac{\pi}{m}$
$m$
0
0
0

م

- Obs

- MORDOH vo

MORDOHV1

TD

- Ana

Nov

Nov Jan

Mar

Year 1996

$\frac{\pi}{0}$
m
$\xi$
3
0
0
0
0
0

- Obs

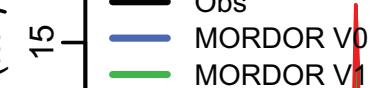

으-- MORDOR Sp

s-

Nov

Jan

Jan Mar

Year 1997

0
m
ह
3
0
0
$\frac{1}{4}$
0
0
0

Obs
MOR

- MORDOR V0

u

(n)

Nov

$M(M$

L

Jan

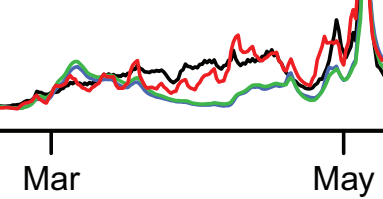

Year 1998

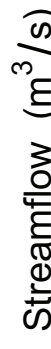
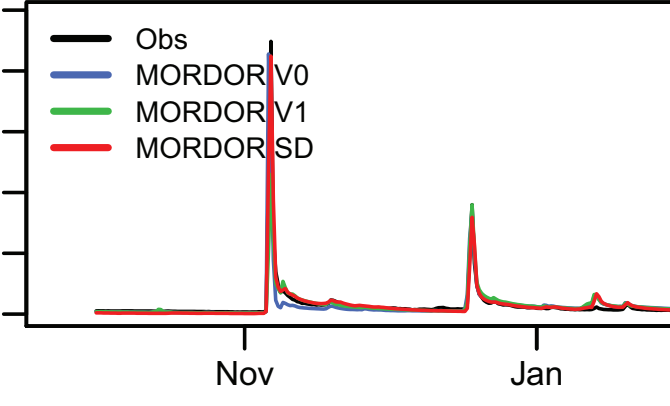

Jan

Mar
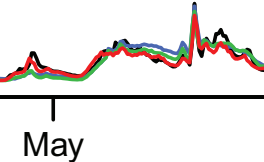

Sep

Year 1999
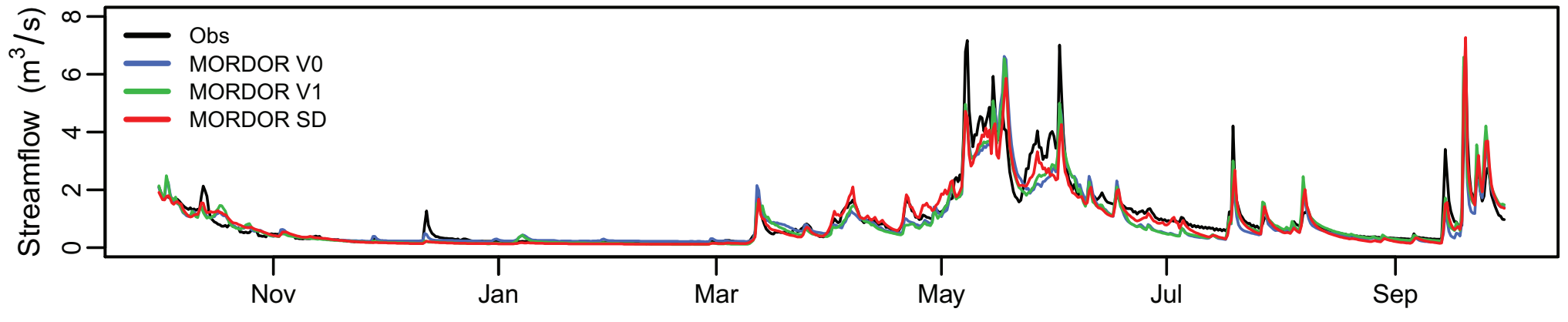
GaveEstaube@Gloriettes

Year 2000

क

0
$\infty$
+
$v$
0
0

- Obs

- MORDOR V1

MORPOR SD

Nov

Nov Jan

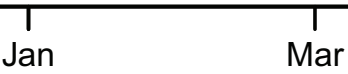

Mar May

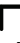

Year 2001
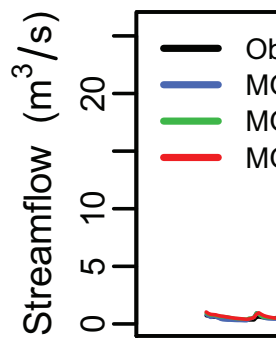

Obs

MORDOR V0

RDOR $V 1$

OR SD

lu

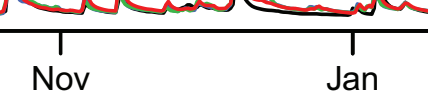

Jan

Year 2002

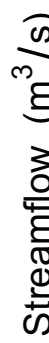

- Obs
- MOkDOR V0
MORDOR V1
MORDOR SD

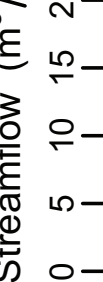

Nov

Jan Mar

Year 2003

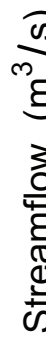

- Obs
MORDOR Vo
MORDOR V

MORDOR V1
MORDOR SD

$M \cup$

Nov

Jan
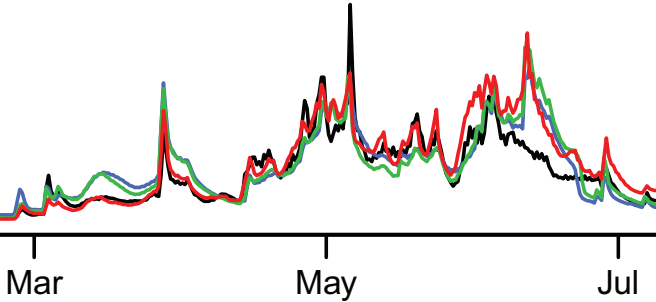

Jul

Jul Sep

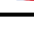

Year 2004

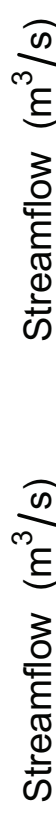

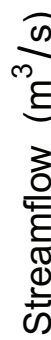

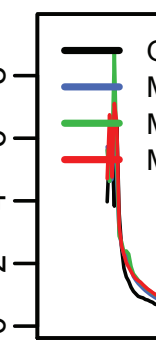

Obs

MORDOR VO

MORDOR V1

MORDOR SD

11

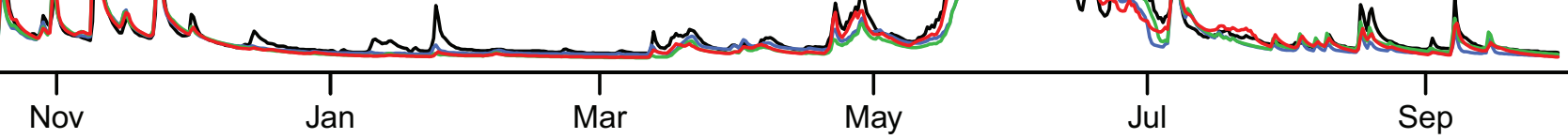

Year 2005
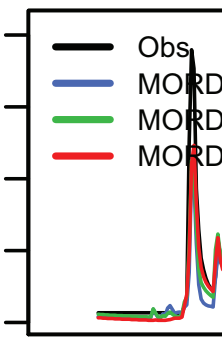

DOR V0

ROR SD

1 
GaveEstaube@Gloriettes

Year 2006

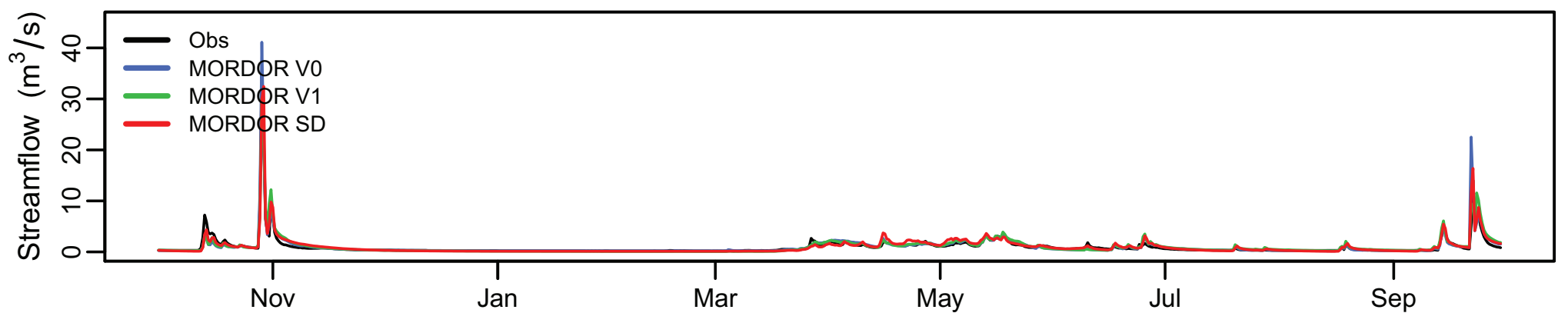




\section{Loire@Basset}

Year 1987
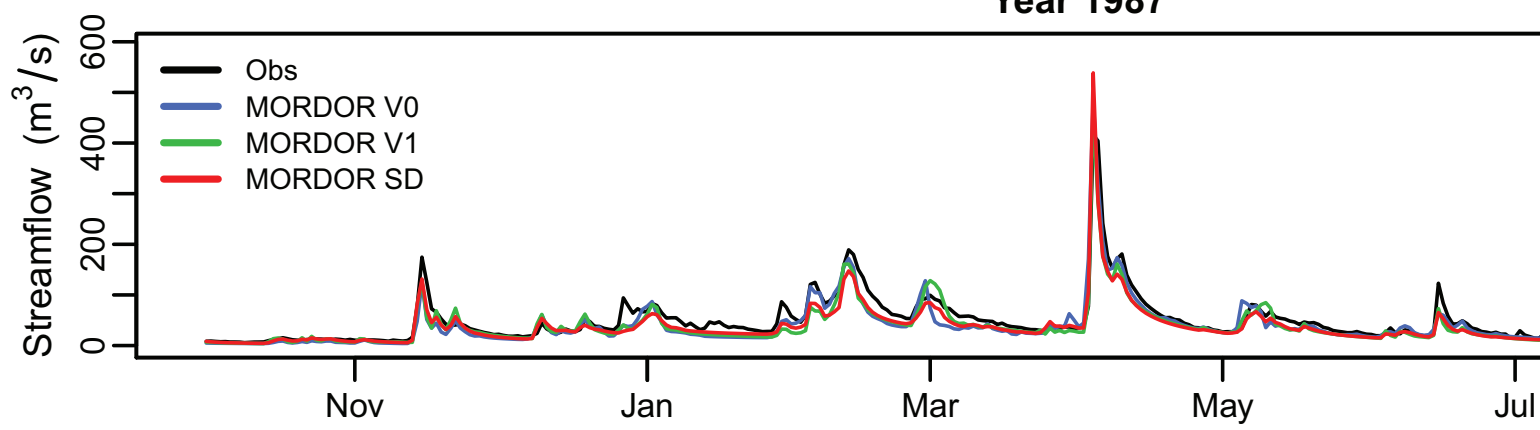

Year 1988
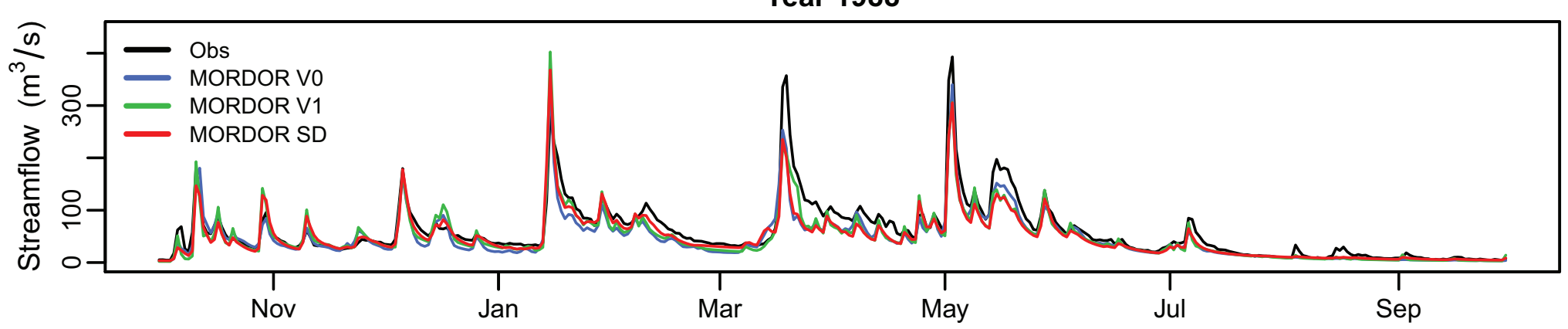

Year 1989

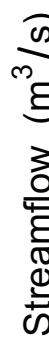
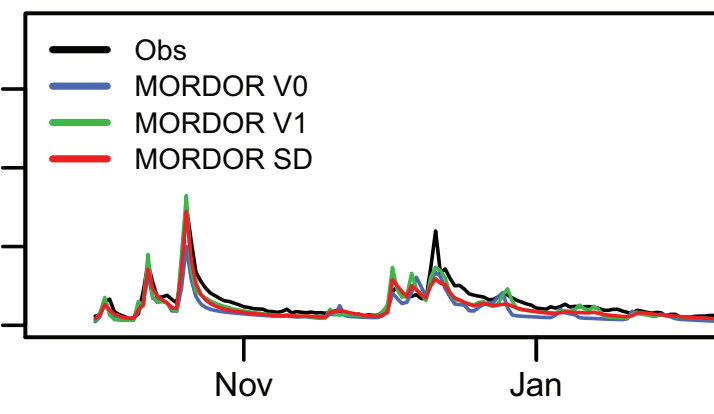

Year 1989 


\section{Loire@Basset}

Year 1993

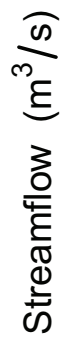

O Obs
- MORDOR V0
MORDOR V1

- MORDOR SD

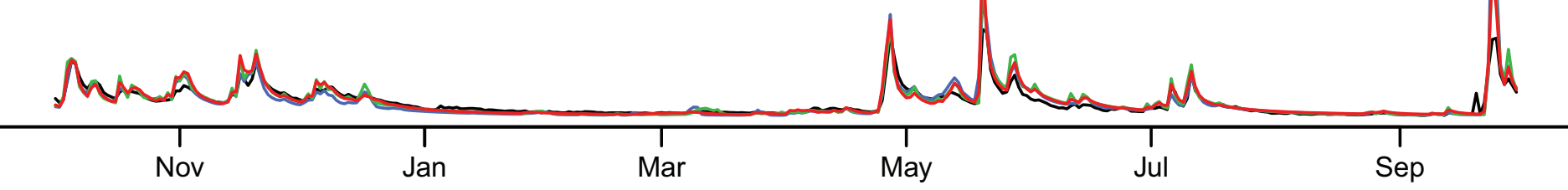

Year 1994

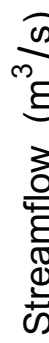

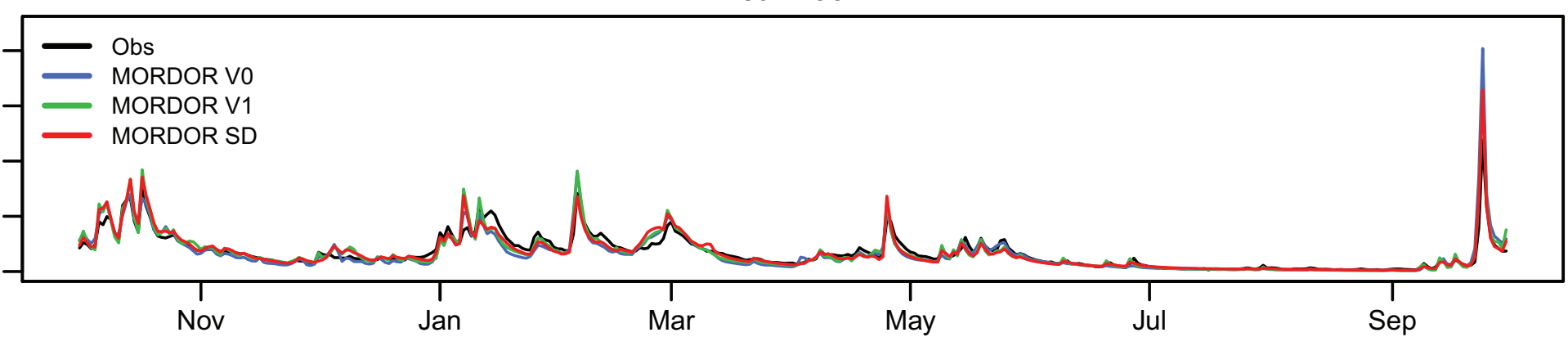

Year 1995

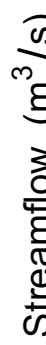

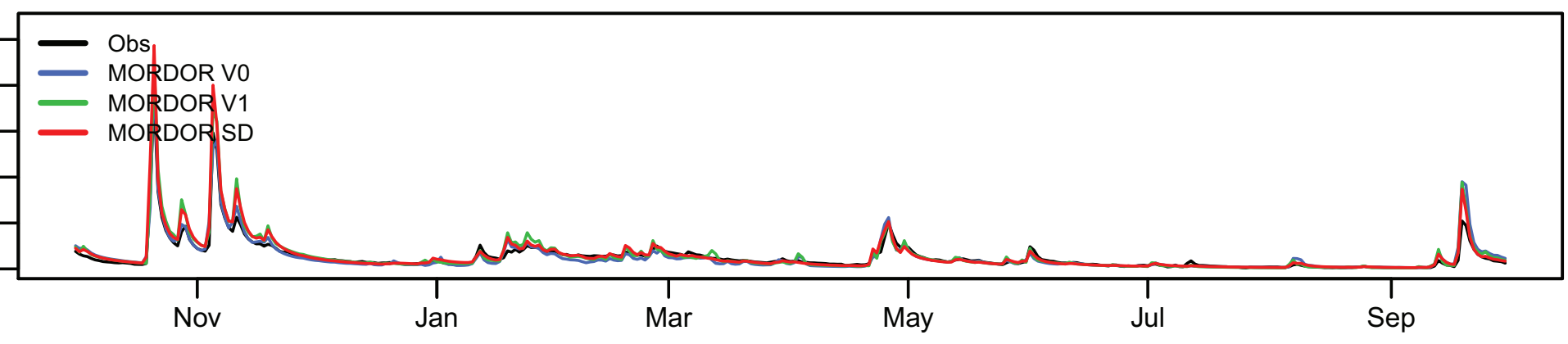

Year 1996

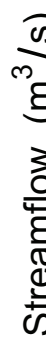

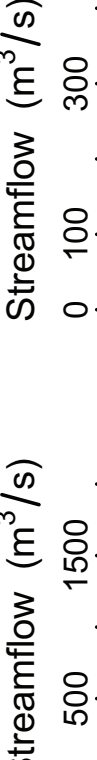
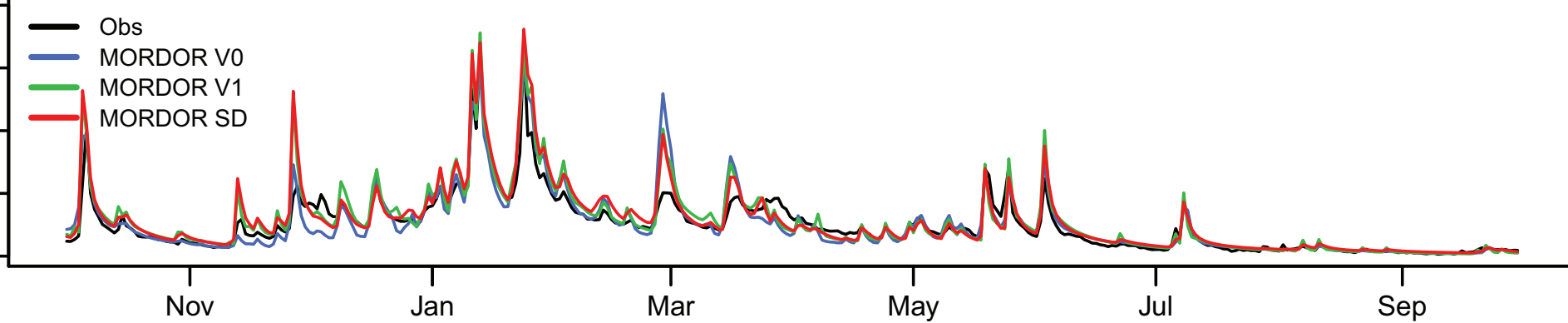

Year 1997
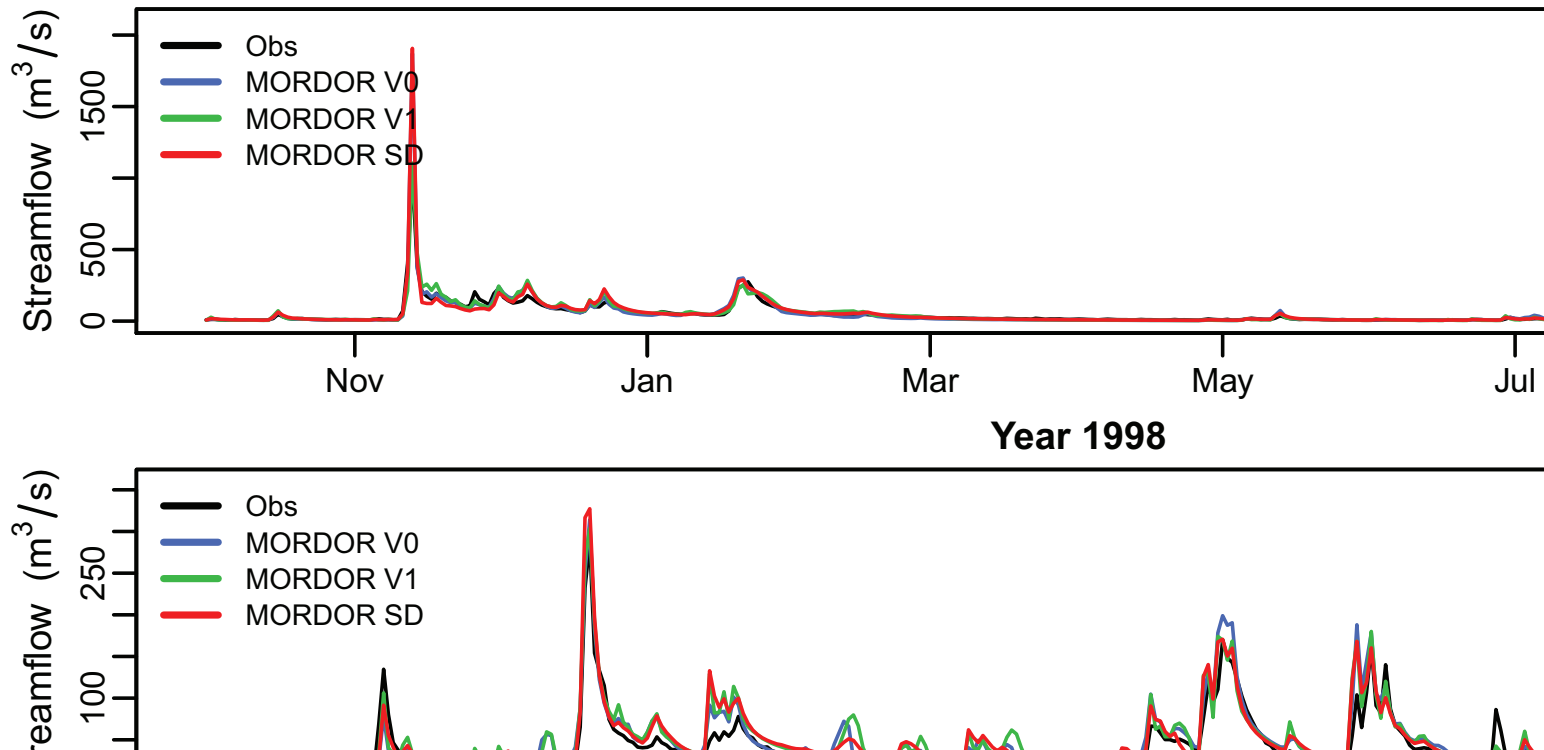

Sep

Year 1998

क

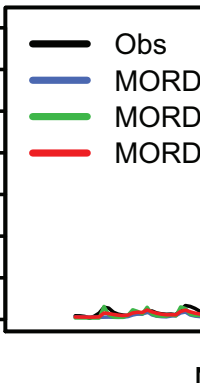




\section{Loire@Basset}

\section{Year 2005}
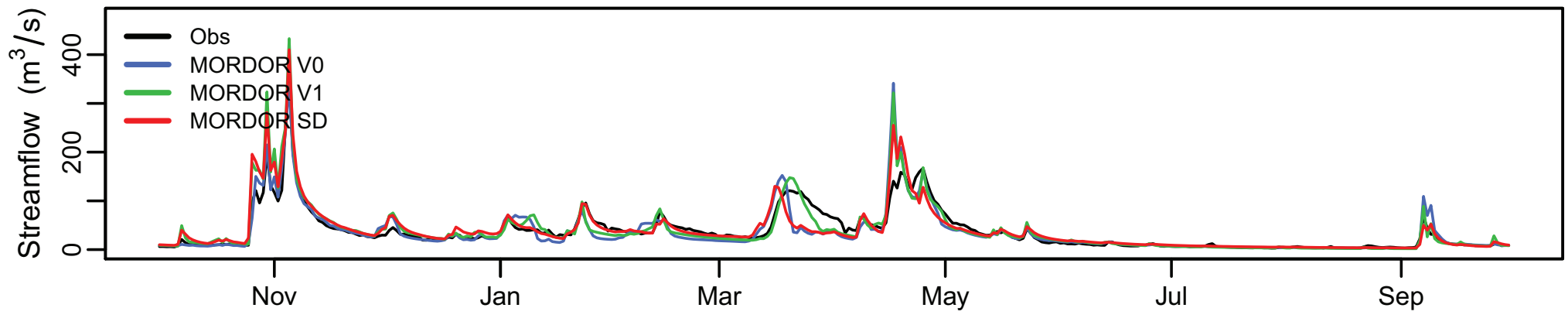

Year 2006

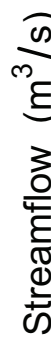

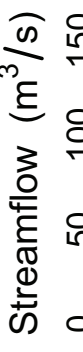

$\longrightarrow$ Obs

$\longrightarrow$ MORDOR V0

MORDOR V1

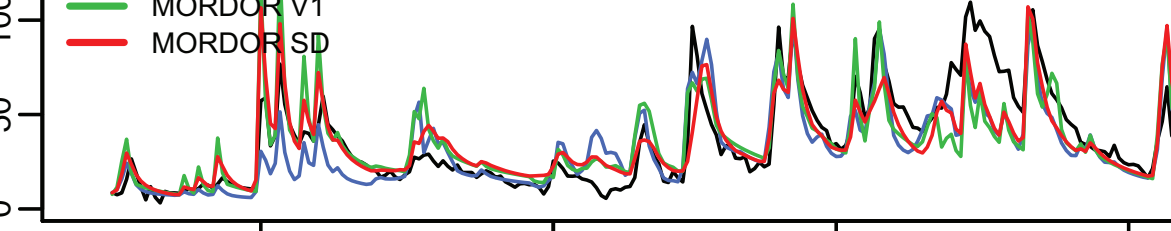

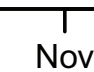

Jan

Mar

Year 2007

क
m
$\xi$
3
30
0
0
0
0
0

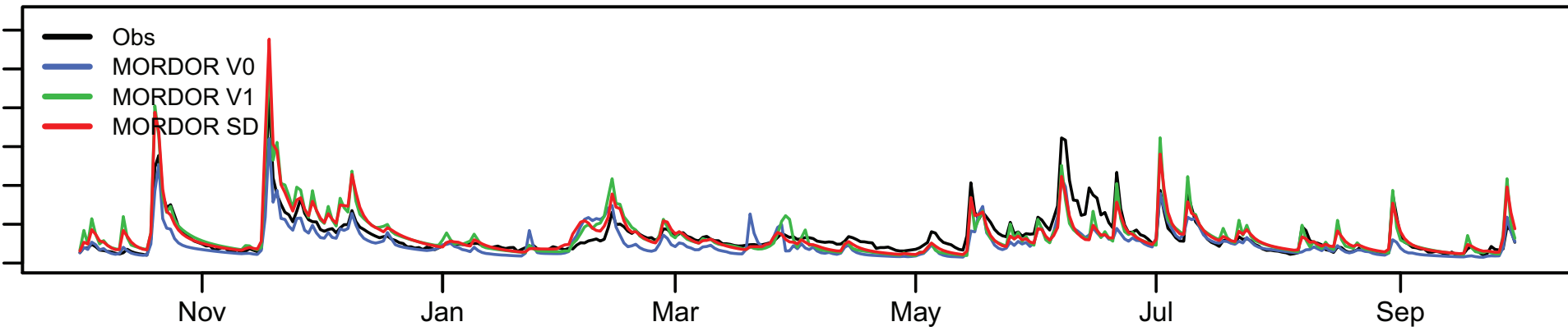

Year 2008

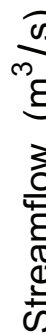

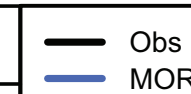

- MORDOR V0

- MORDOR V1

$-\longrightarrow$ MORDOR SD

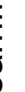

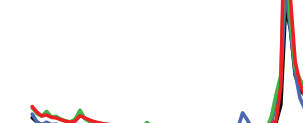

Nov Jan

Jan

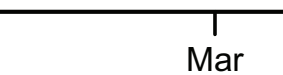

Mar

Year 2009
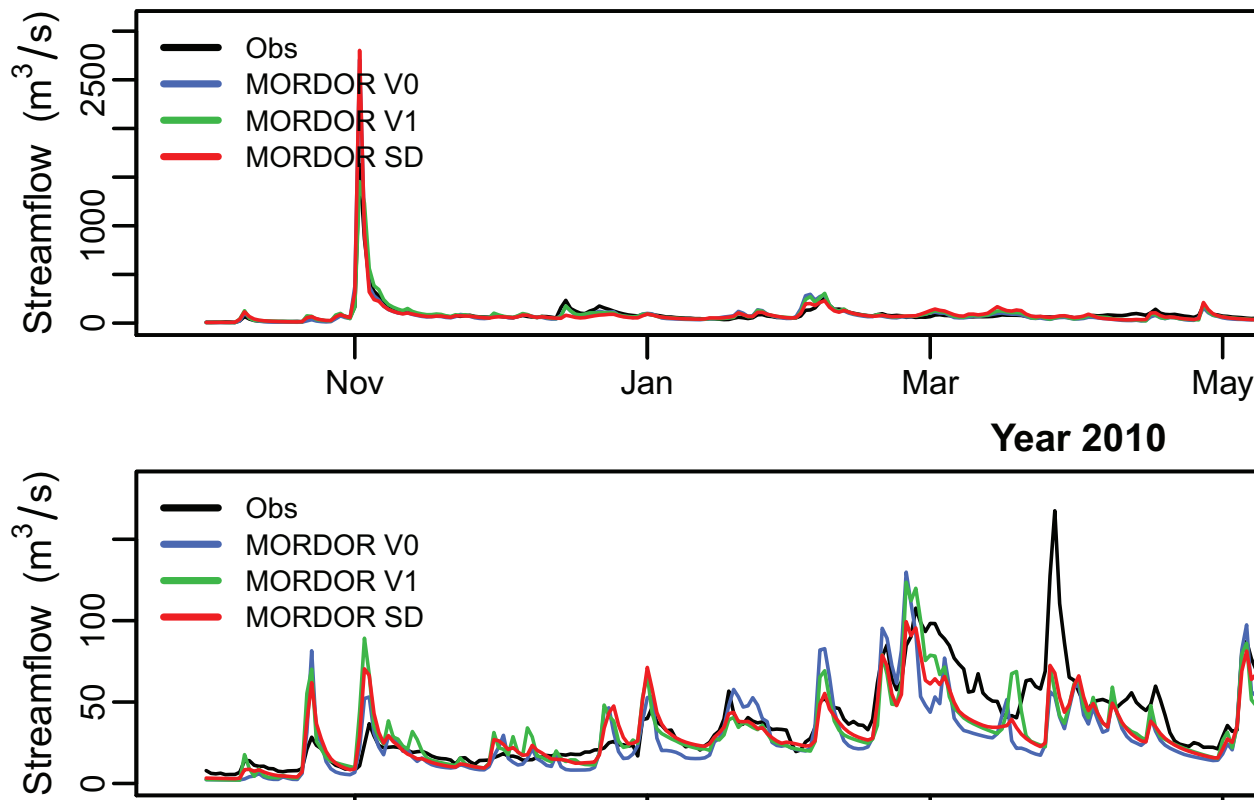

Jul

Year 2010

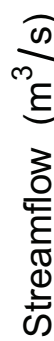

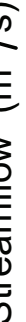

$$
\text { 正 }
$$$$
\text { Obs }
$$$$
\text { MORDOR V0 }
$$$$
\text { MORDOR V1 }
$$$$
\text { MORDOR SD }
$$ 


\section{Mimente@Florac}

Year 1986
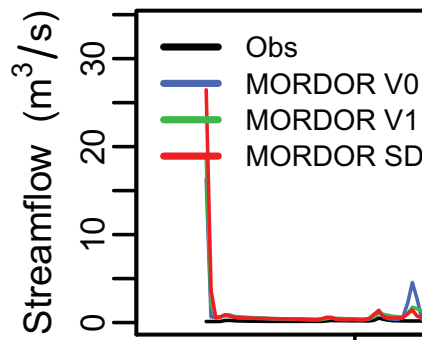

Year 1986

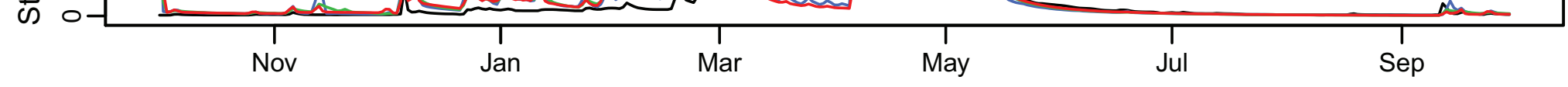

Year 1987
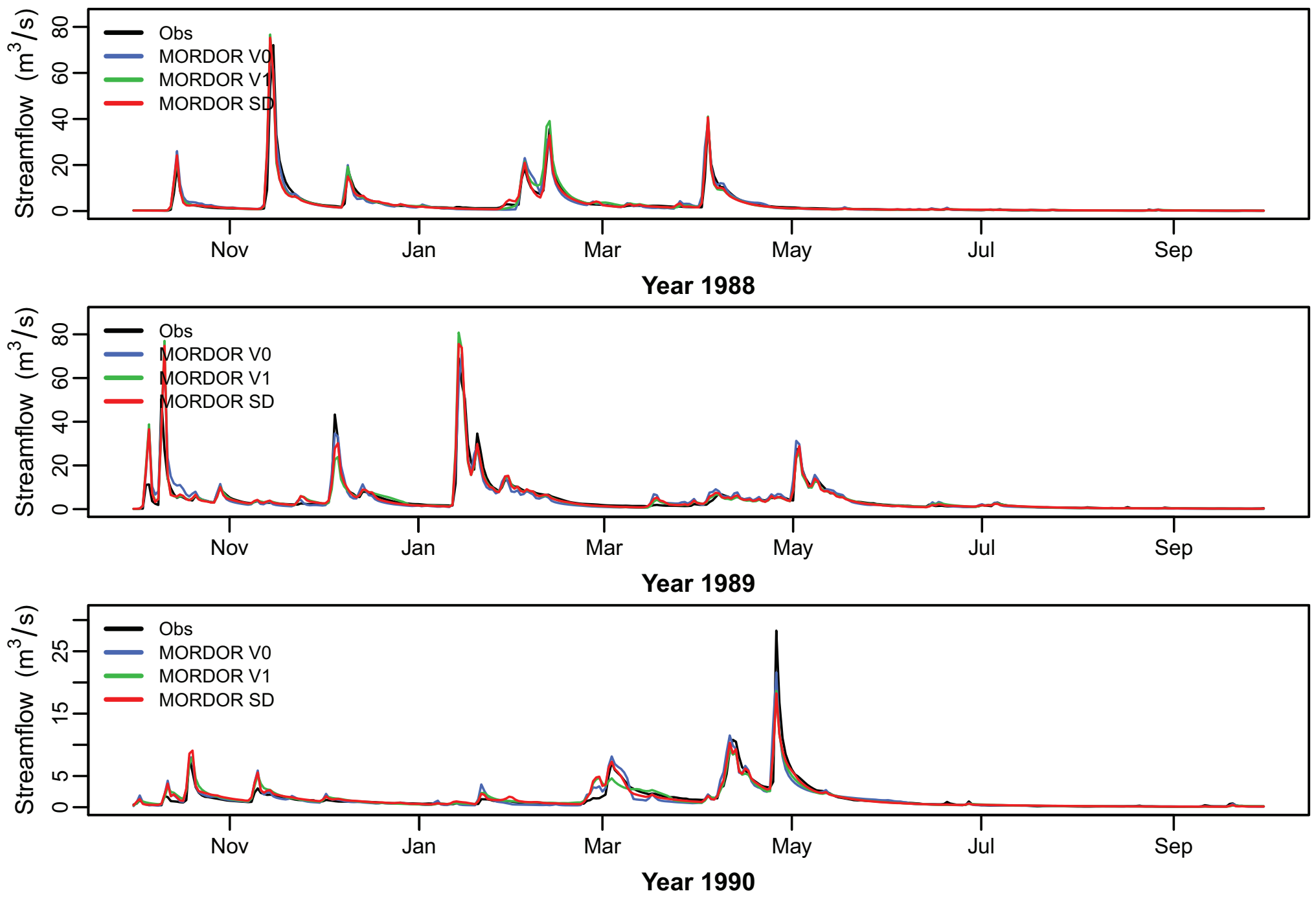

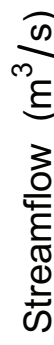

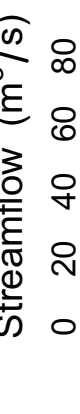

1990

- Obs

MORDOR V

$\longrightarrow$ MORDOR SD

O
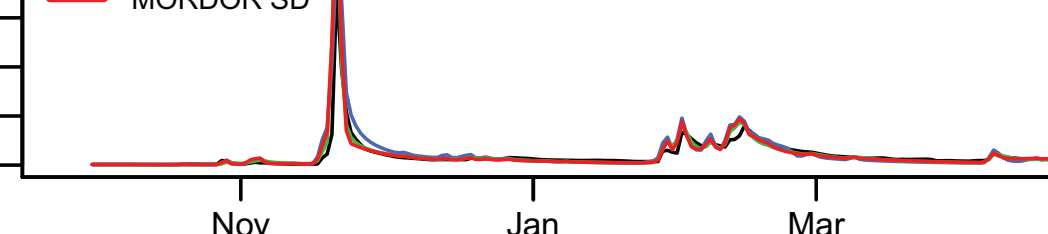

A

Mar May

Jul

Sep

Year 1991

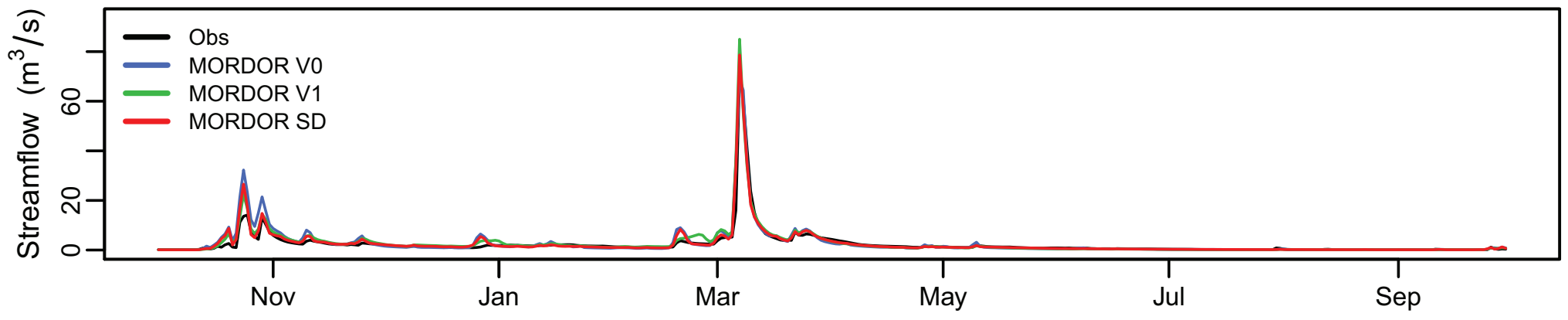




\section{Mimente@Florac}

Year 1992

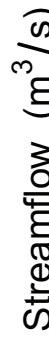

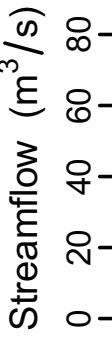

$\longrightarrow$ Obs

$\longrightarrow$ MORDOR V0

- MORDOR V1

MORDOR SD

.
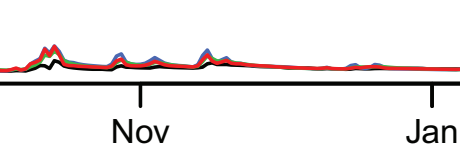

Mar

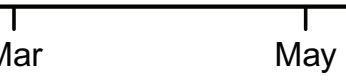

Year 1993
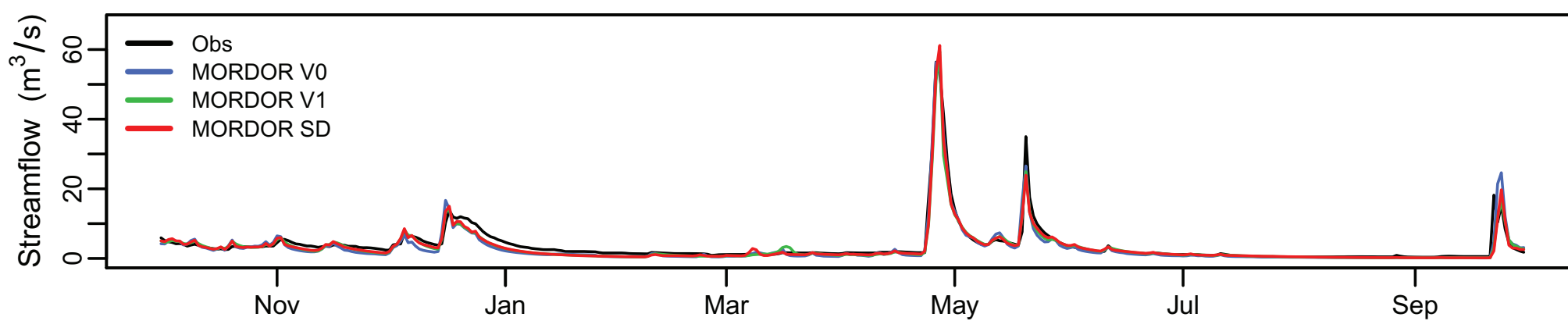

Year 1994

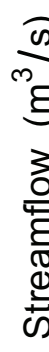

- Obs
MORDOR V0
MORDOR V1

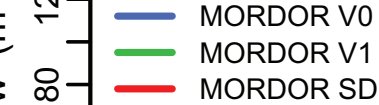

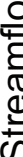

o-
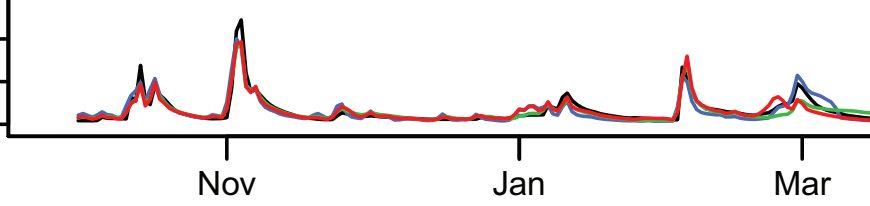

Year 1995
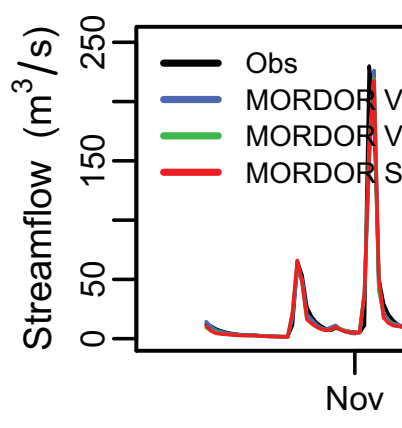

- Obs

- MORDOR VO

MORDOR V1

MORDOR SD

- limula N

Nov

Jan

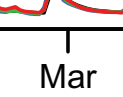

Year 1997
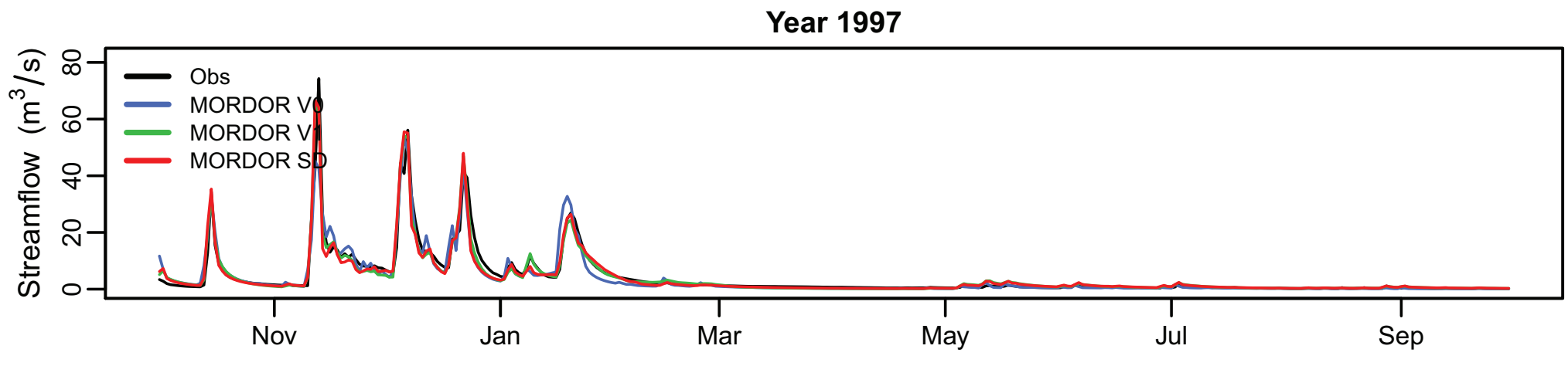
Montane@Eyrein

Year 1959

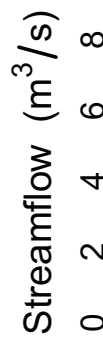

Obs

- MORDOR VO

— MORDOR V1

- MORDOR SD

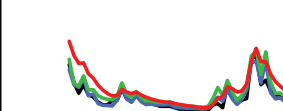

Nov
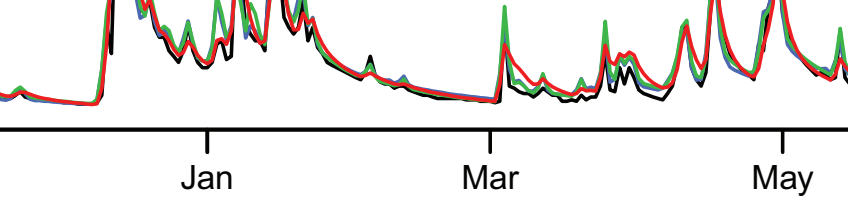

Year 1960
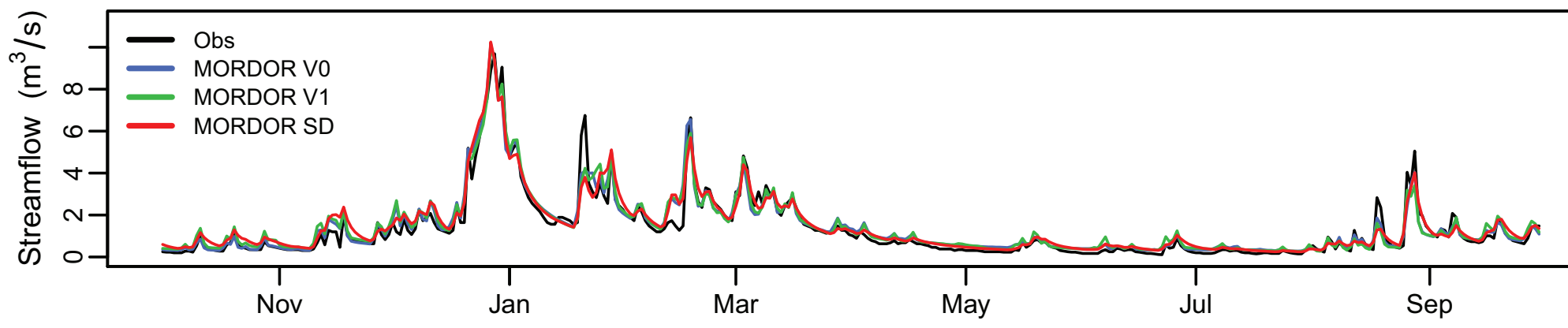

Year 1961
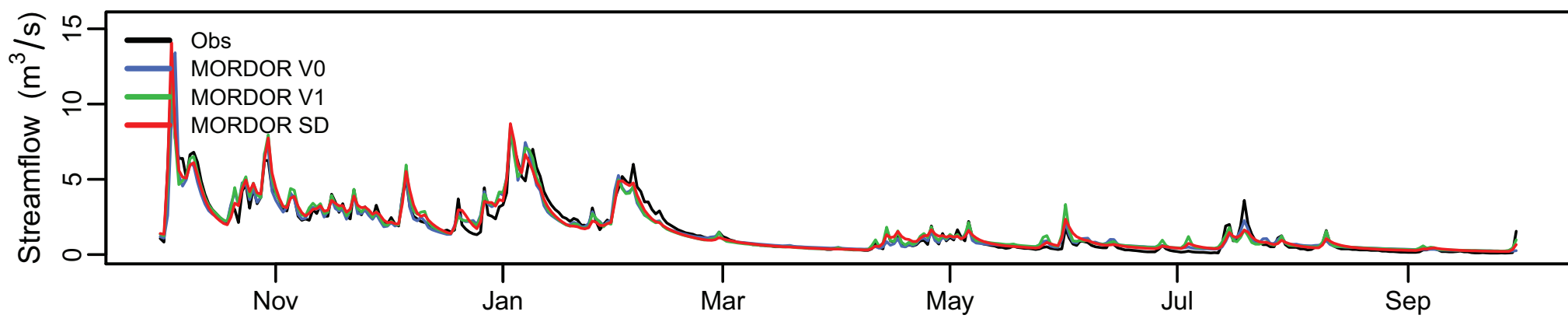

Year 1962
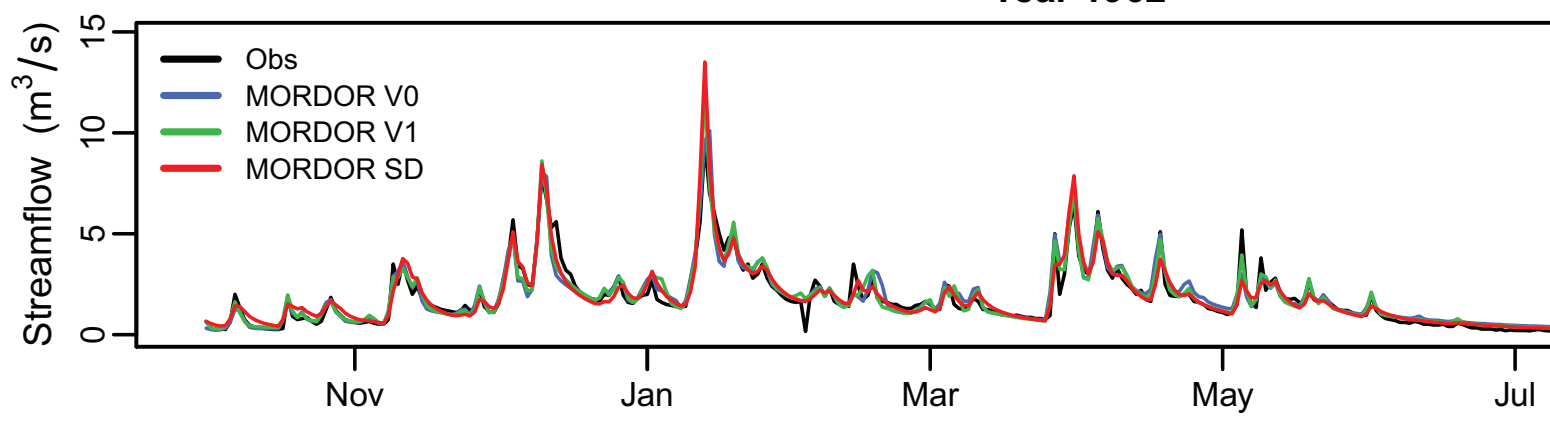

Na

Year 1963
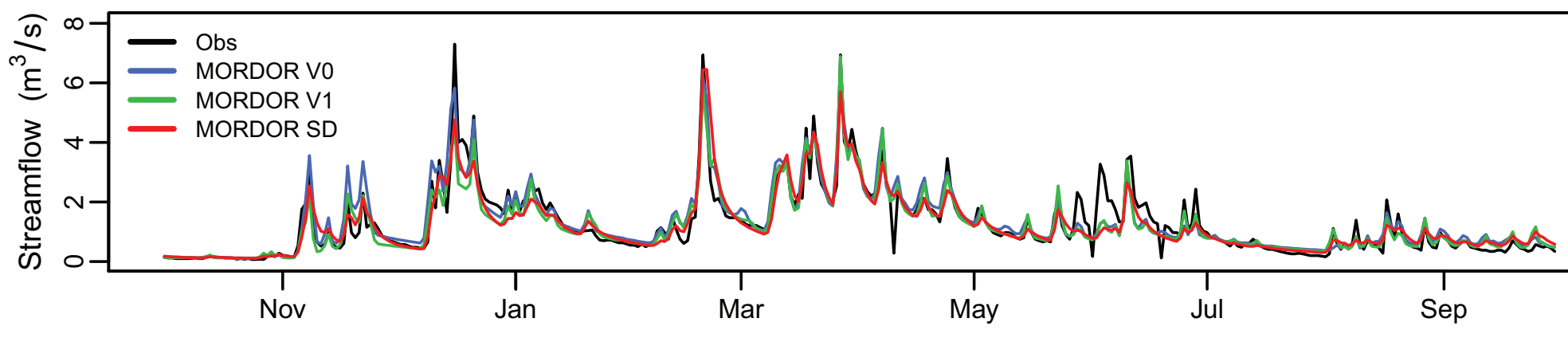

Year 1964
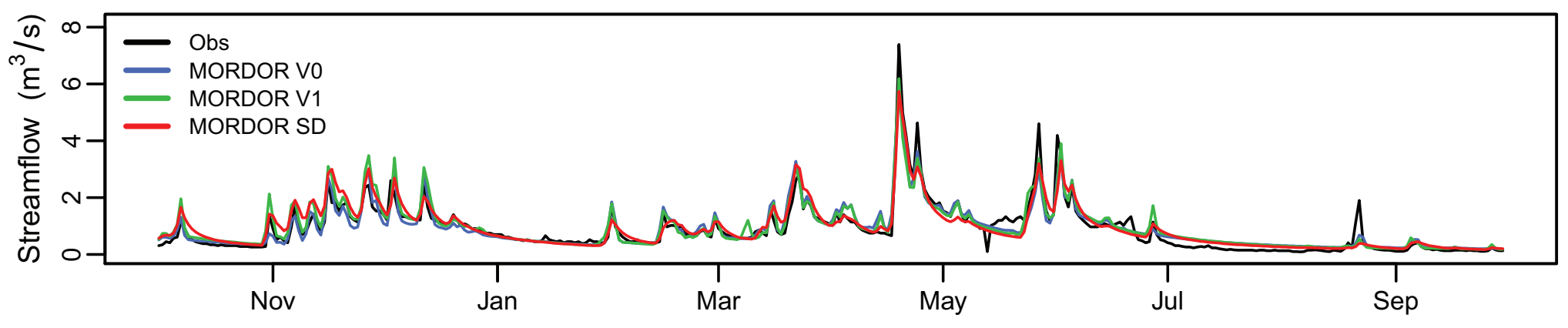
Montane@Eyrein

Year 1983

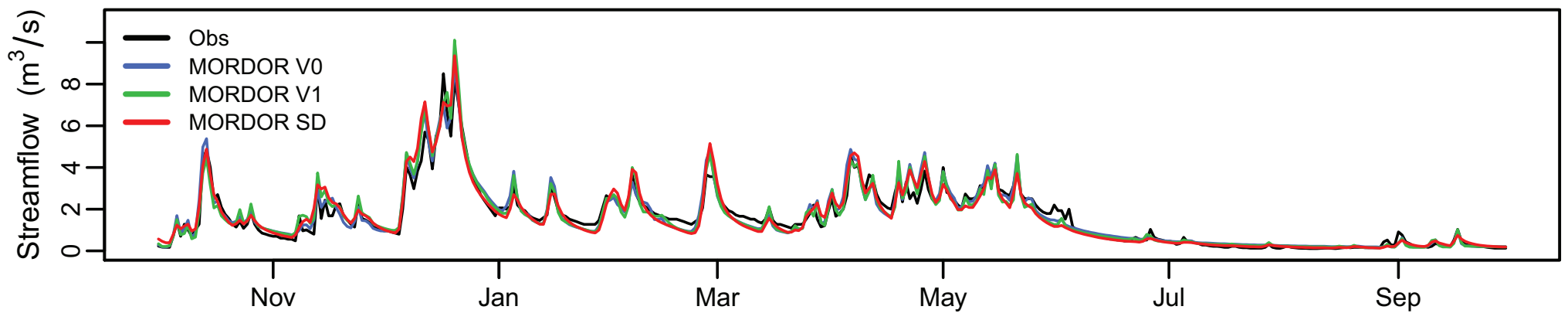

Year 1984
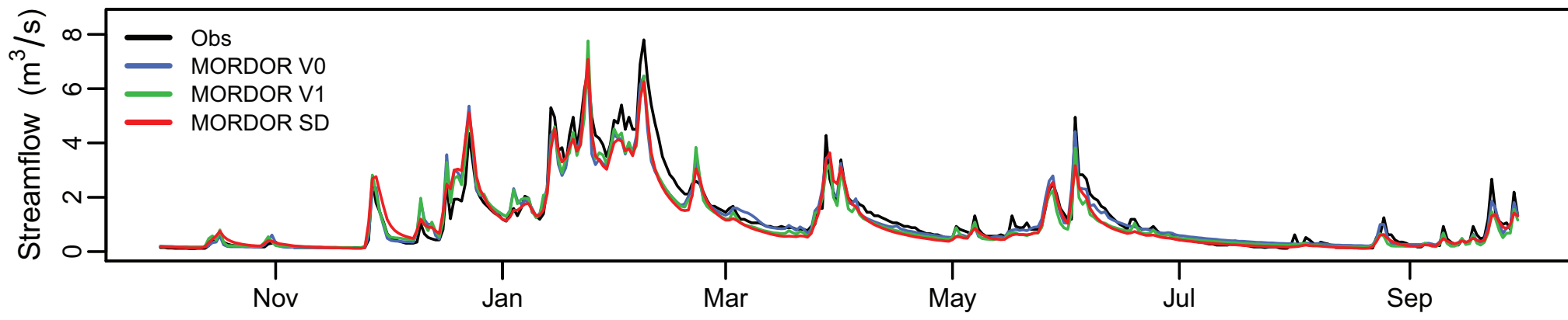

Year 1985

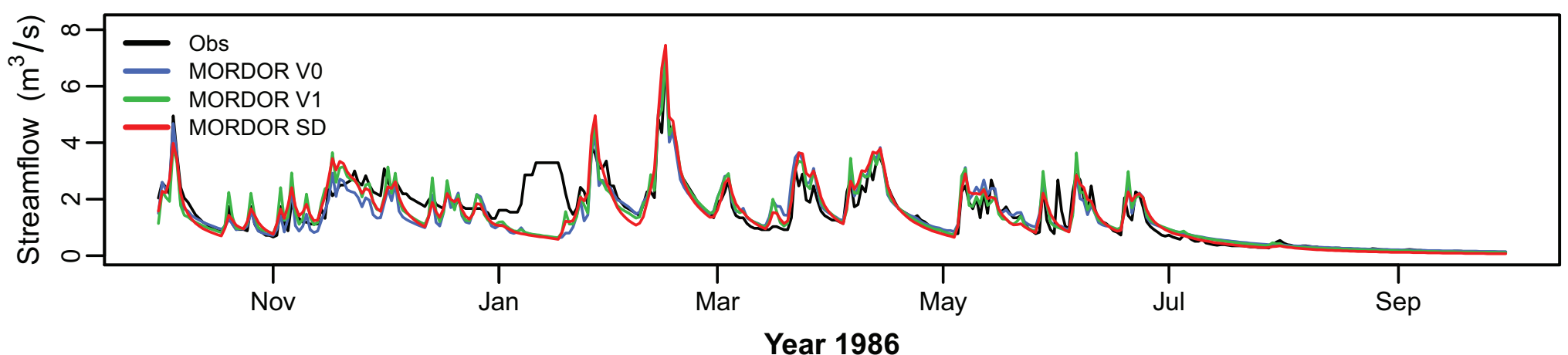

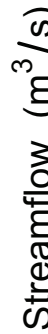

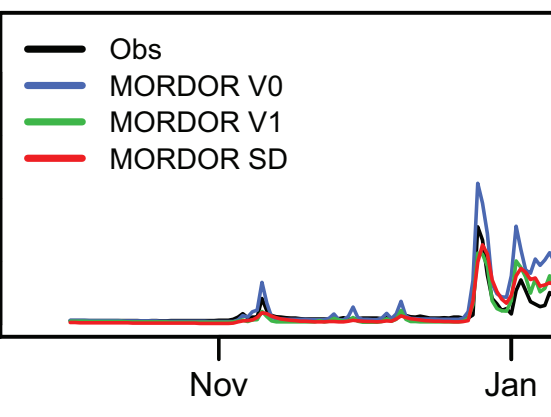

Year 1986 
Montane@Eyrein

Year 1989

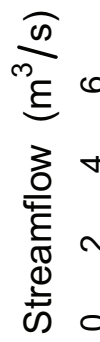

Obs

MORDOR Vo

MORDOR V1

— MORDOR SD
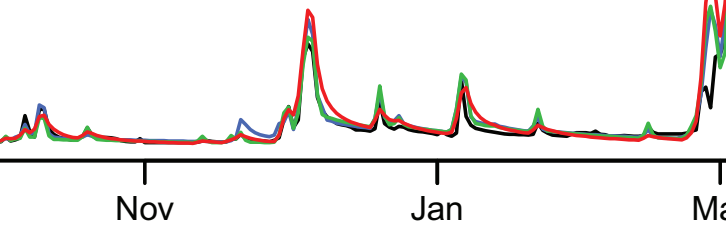

Mar May

Year 1990

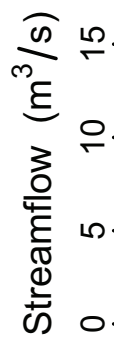

- Obs

- MORDOR V0

- MORDOR V1

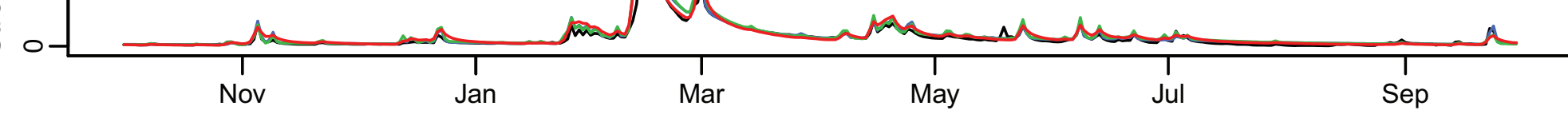

Year 1991

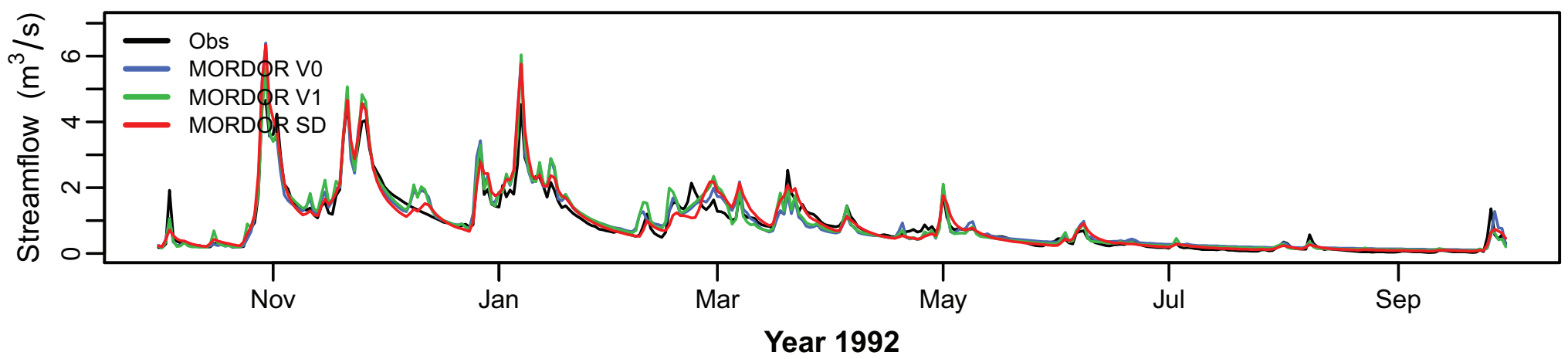

$\frac{\pi}{m}$
m
$\xi$
3
0
5
0
0
0

- Obs

- MORDOR Vo

- MORDOR SD

Mas

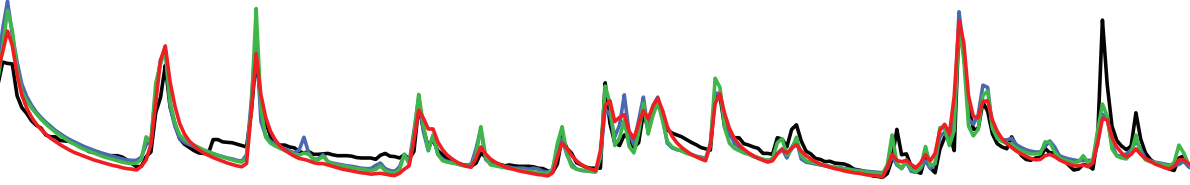

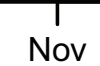

Jan

Mar

May

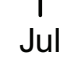

Year 1993

0
m
है
3
0
0
0
0
0
0

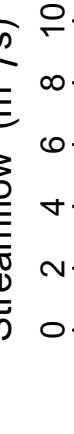

$-$

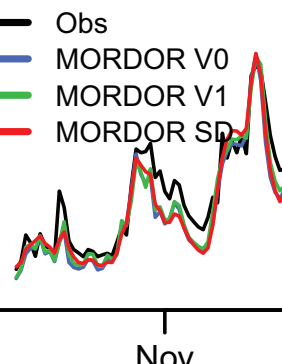

Nov
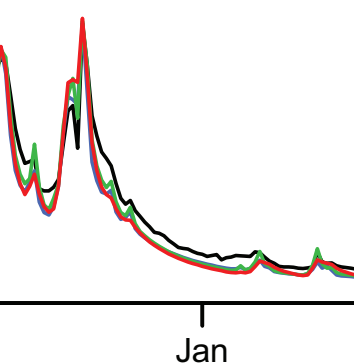

Jul

Sep 


\section{Rizzanese@Barrage}

Year 2000
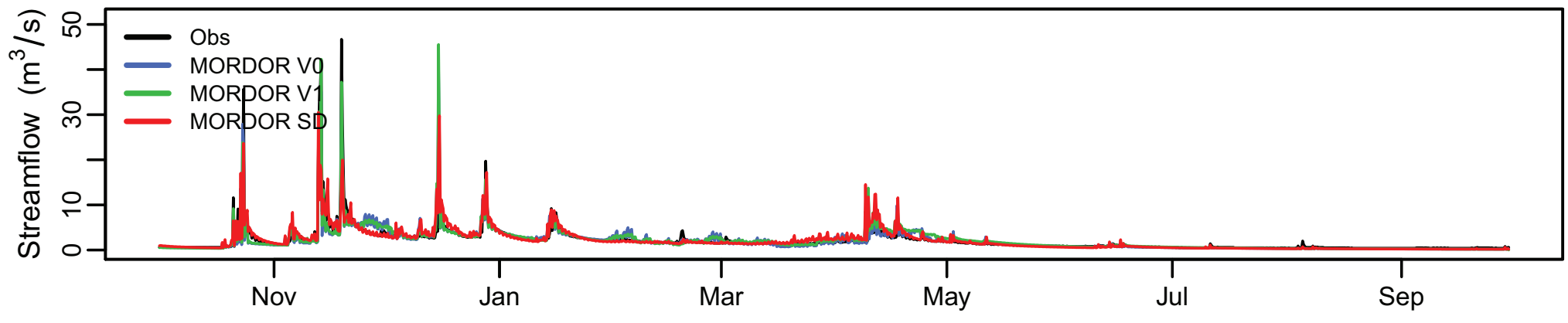

Year 2001
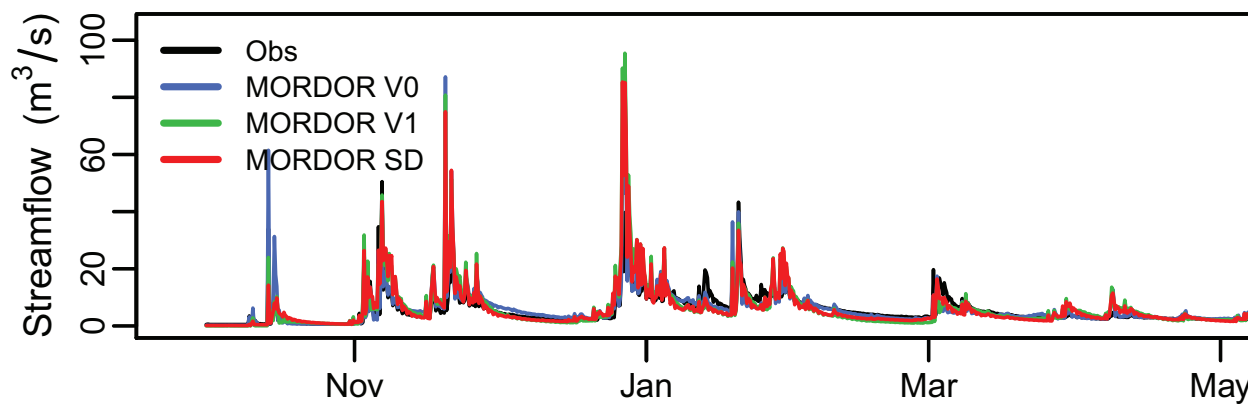

Year 2002

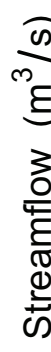

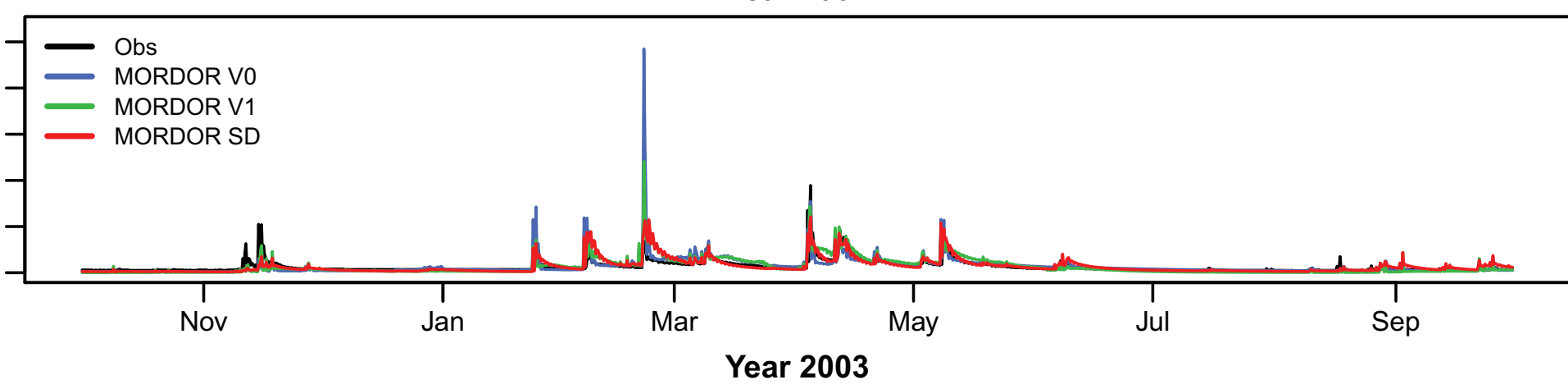

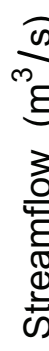

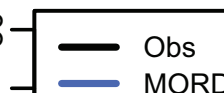

工

MORDOR V1

m- MORDOR SD

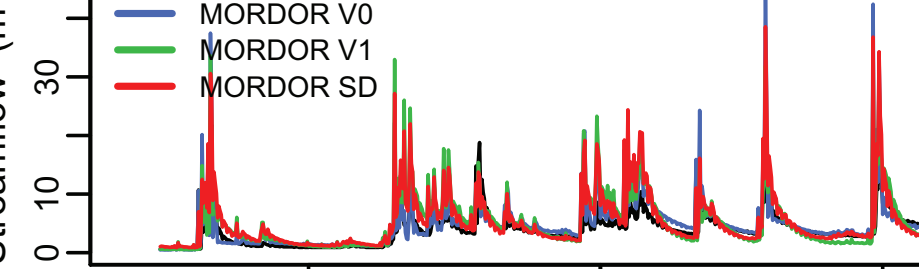

Nov Jan J

Year 2004

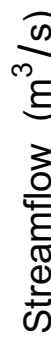

m

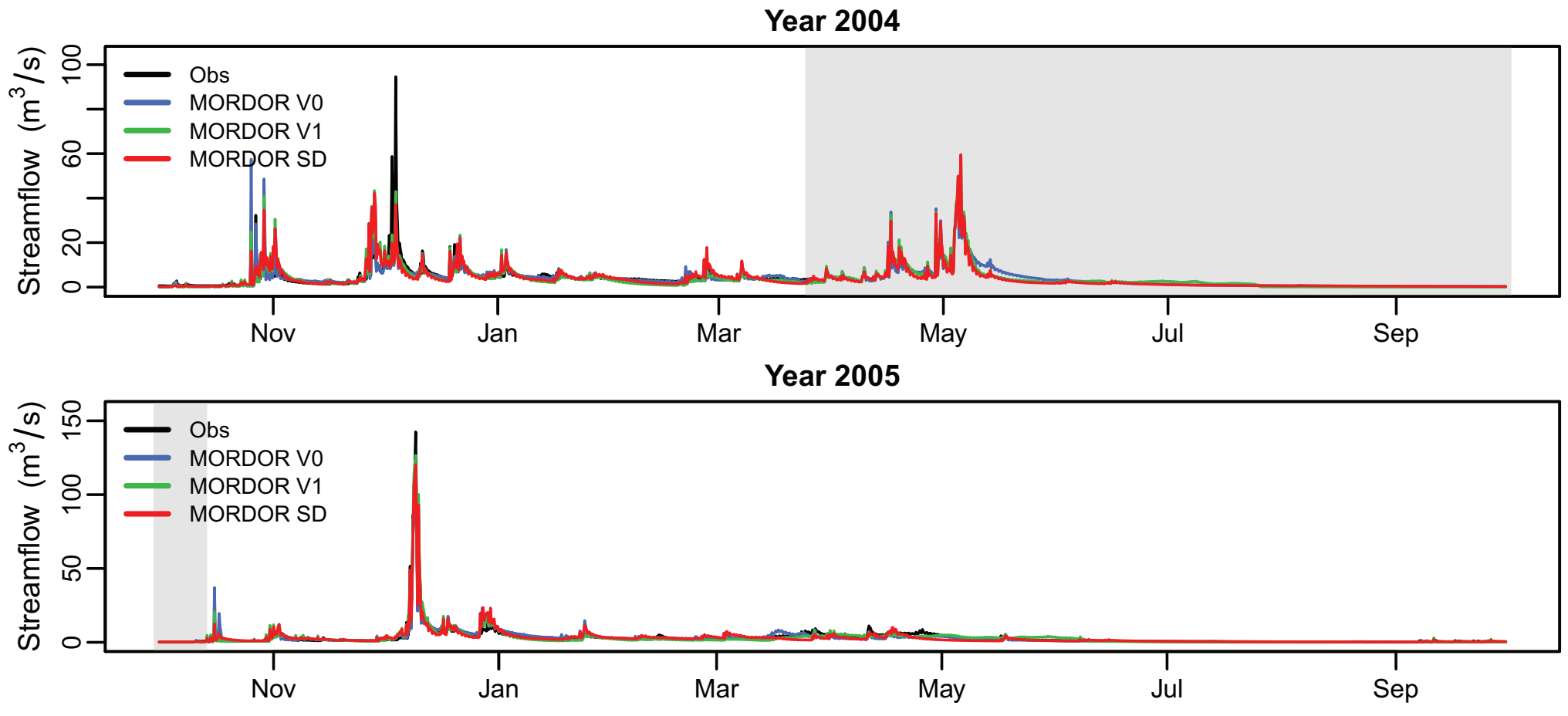



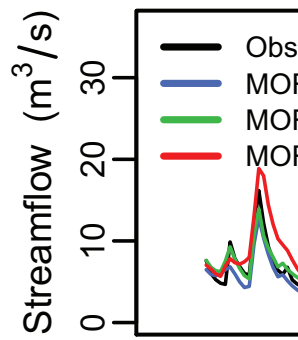

A

MORDOR V0

MORDOR V

- MORDOR SD

으-

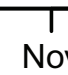

ov

Year 1993
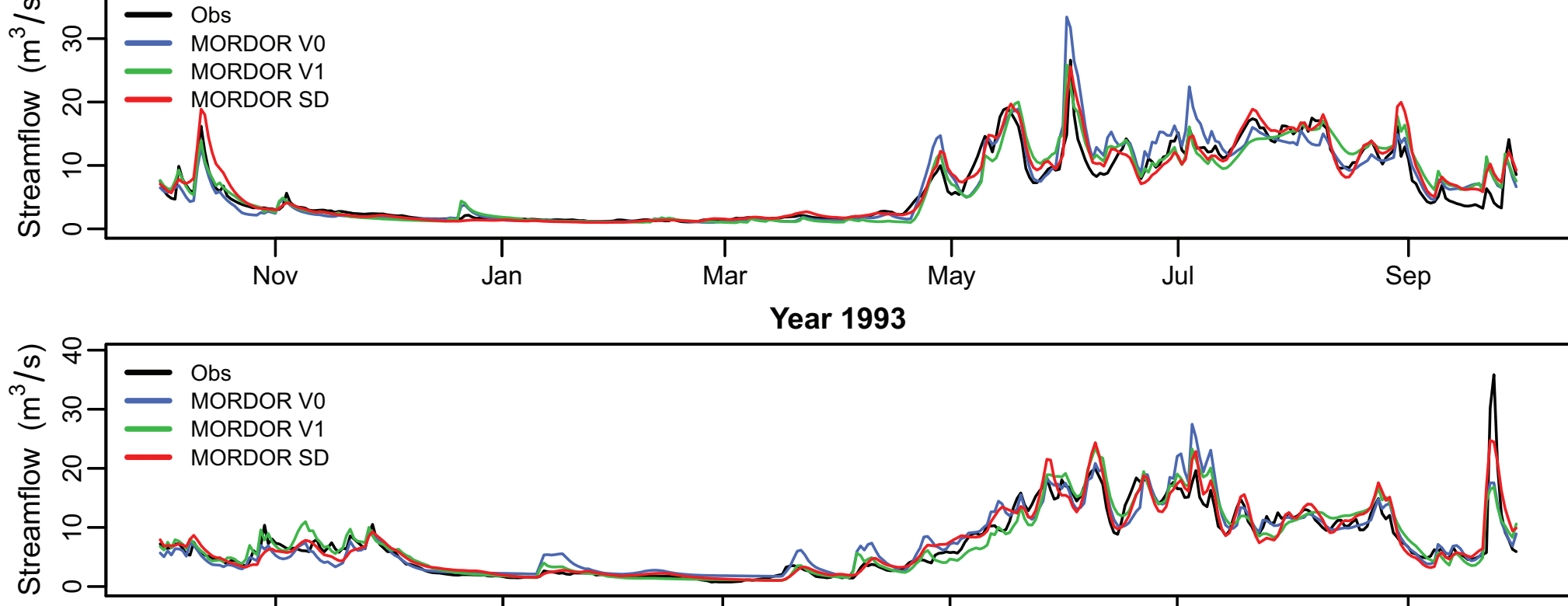

$\longrightarrow$ Obs

$\longrightarrow$ MORDOR V0

$\longrightarrow$ MORDOR V1

$\longrightarrow$ MORDOR SD

Nov

Jan Mar

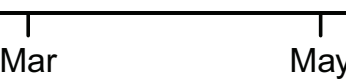

May

Year 1994

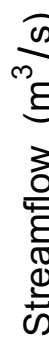

- Obs

- MORDOR V0

- MORDOR V1

MORDOR SD

ㄱ.
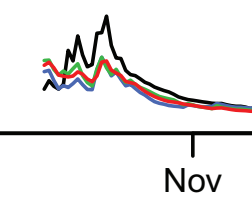

$\Lambda$

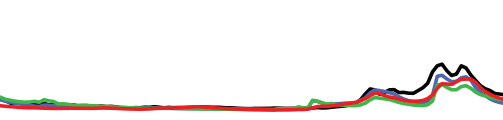

Jan

Mar

Year 1995

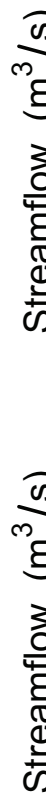

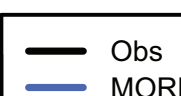

- MORDOR V0

- MORDOR V1

MORDOR SD

ban

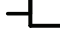
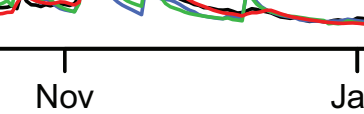

Jan
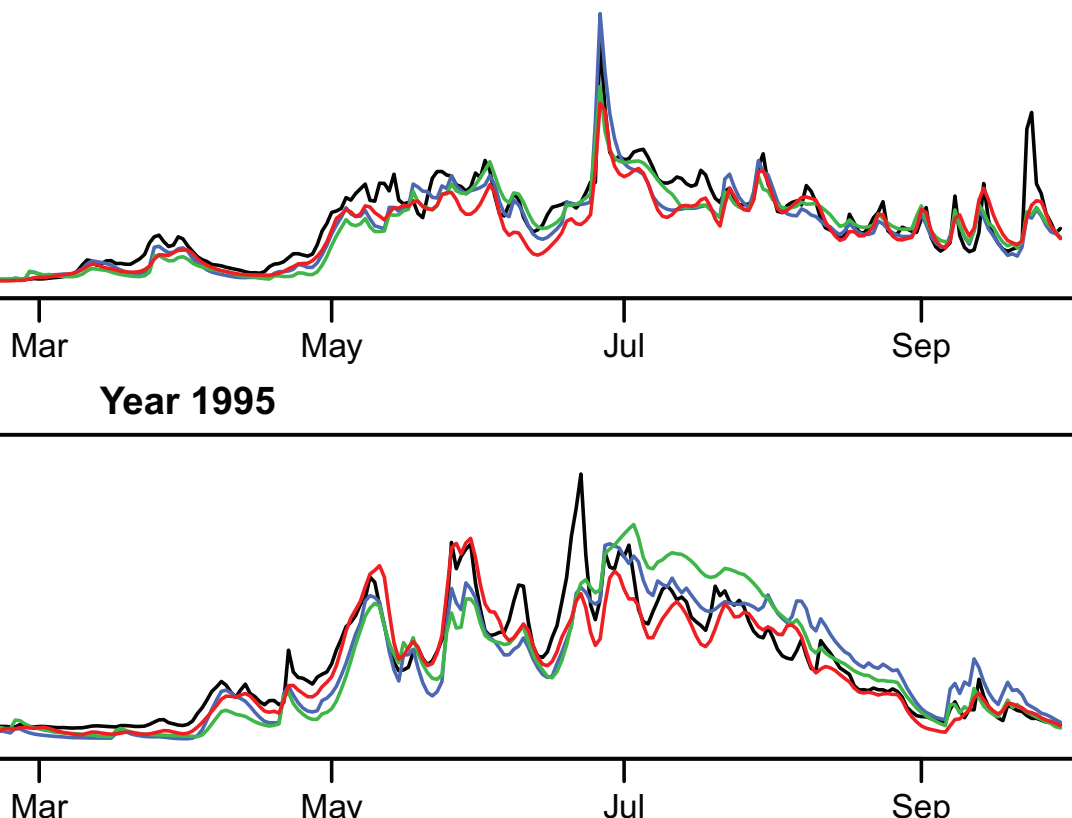

Jul

Sep 
Romanche@Chambon

Year 2004

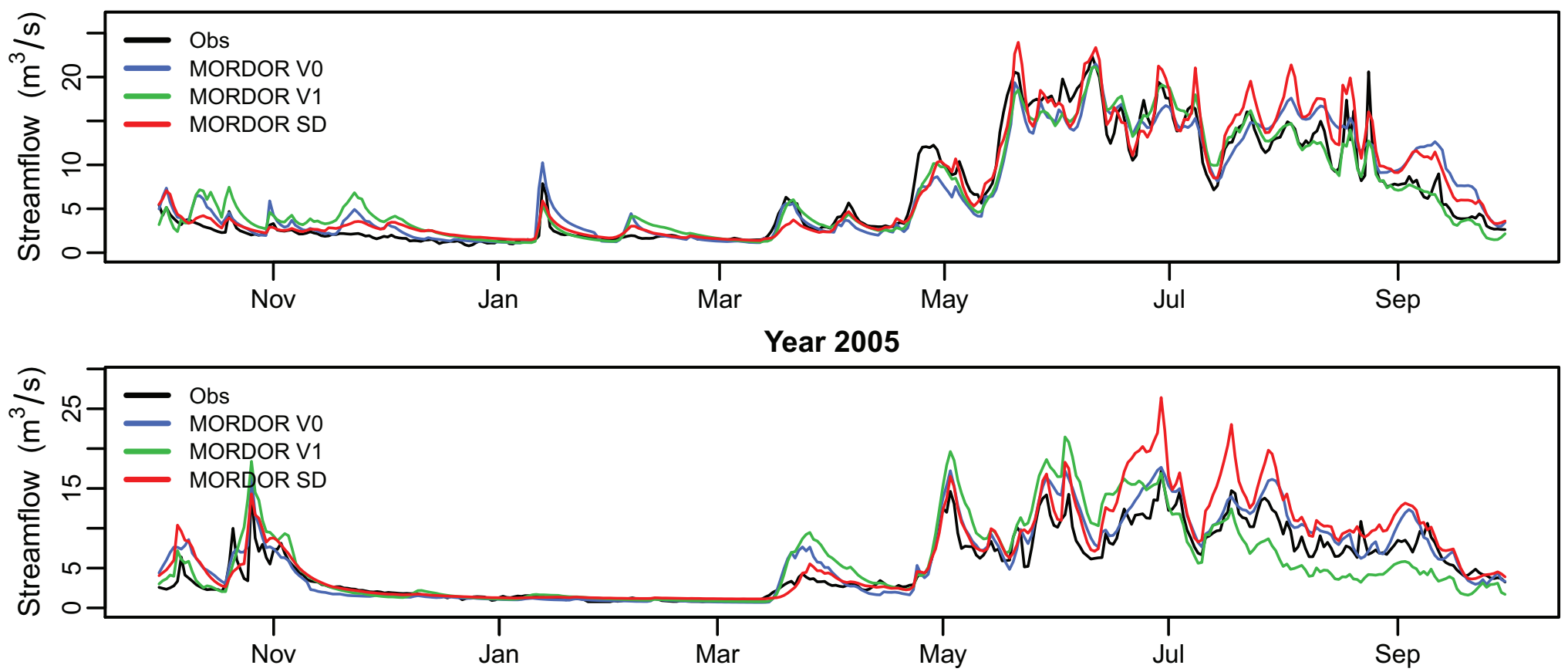


Sioule@Fades

Year 1993
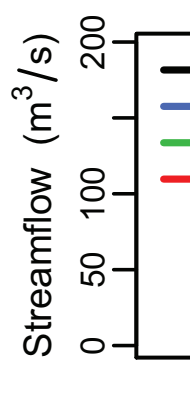

Obs

- MORDOR V0

- MORDOR V1

MORDOR SD

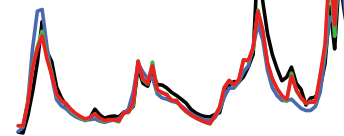

Nov

Jan

Mar

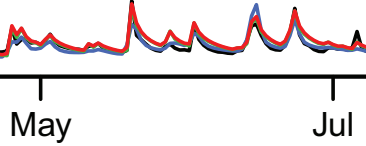

a

Year 1994

क

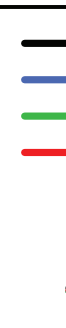

Obs

MORDOR V0

- MORDOR V1

- MORDOR SD

Nher.

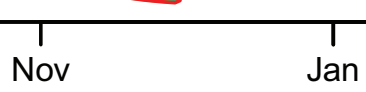

an Mar

Year 1995
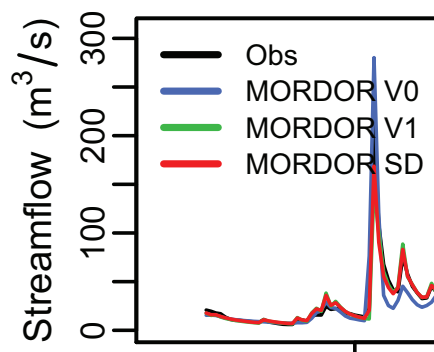

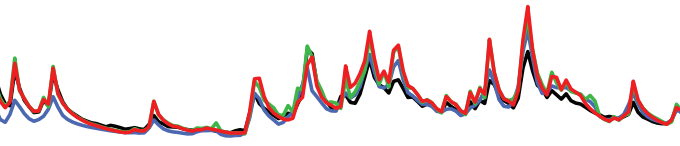

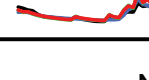

Nov

Jan

Mar

Year 1996

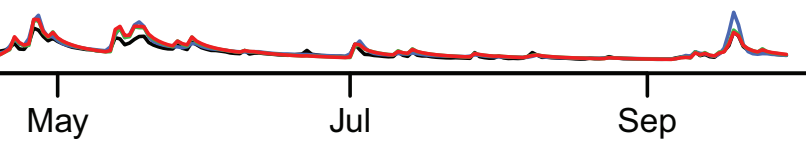

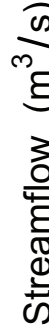

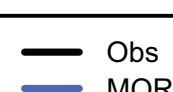

- Obs

MORDOR V0

- MORDOR SD

西

arand

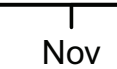

Nov
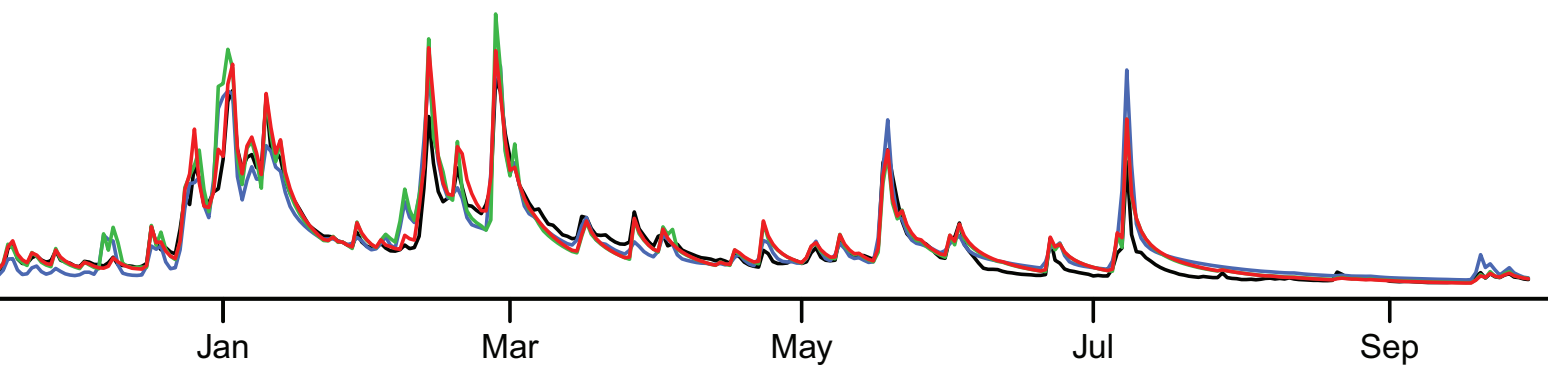

Year 1997

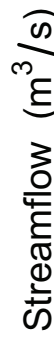

- Obs

- MORDOR VO

MORDOR V1

ס
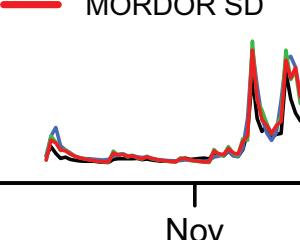

Nov
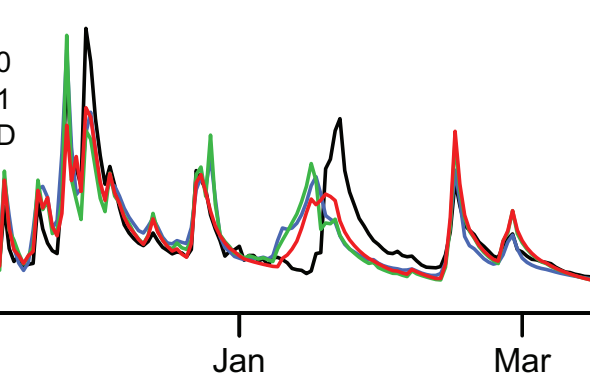

Mn

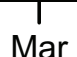

May

Ana

Jul Sep

Year 1998

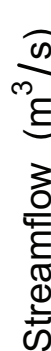

응

- obs

- MORDOR V0

- MORDOR V1

$\longrightarrow$ MORDOR SD

-

Anam M 


\section{Sioule@Fades}

Year 1999
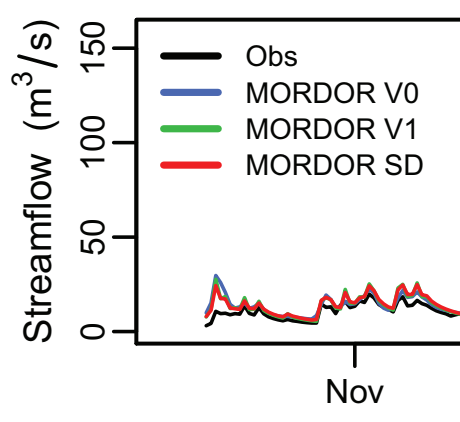

Ammanchand M
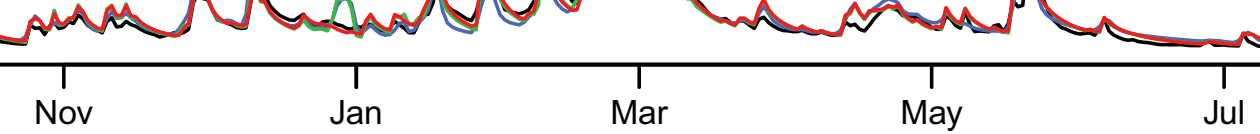

Year 2000
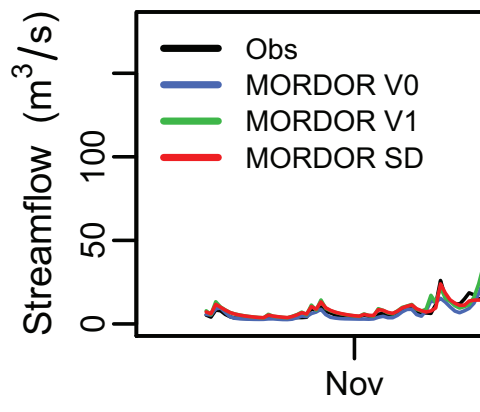

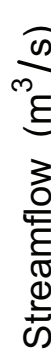

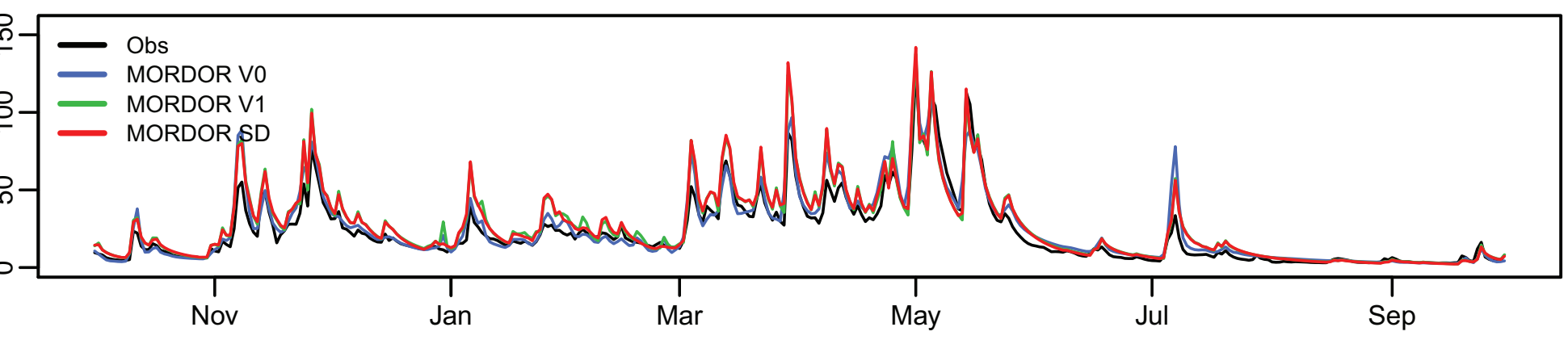

Year 2002
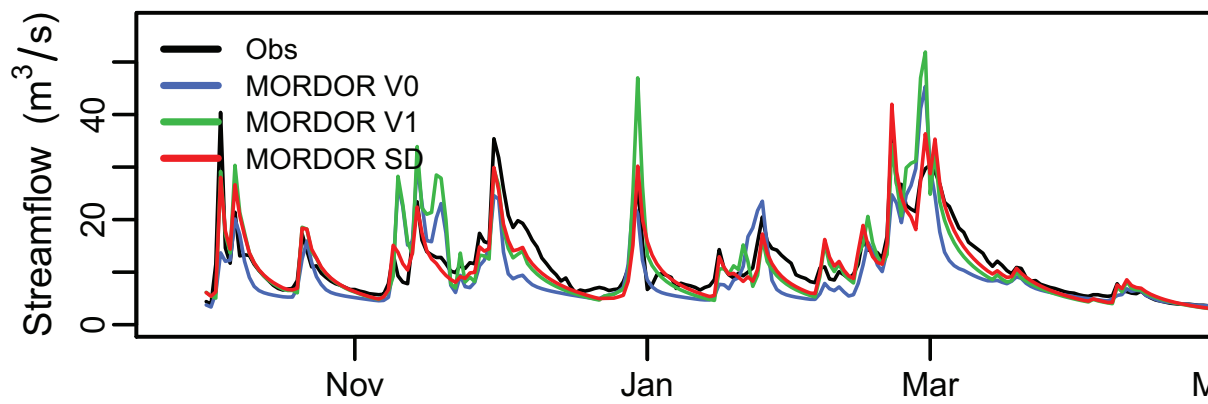

Year 2003

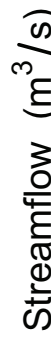

- Obs

- MORDOR VO

$\longrightarrow$ MORDOR V1

ㄴ- - MORDOR SD

ह

政

은
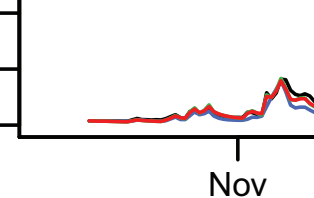

Lan

Jan

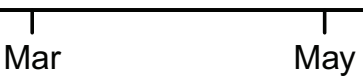

Jul

Year 2004

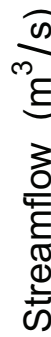

- Obs
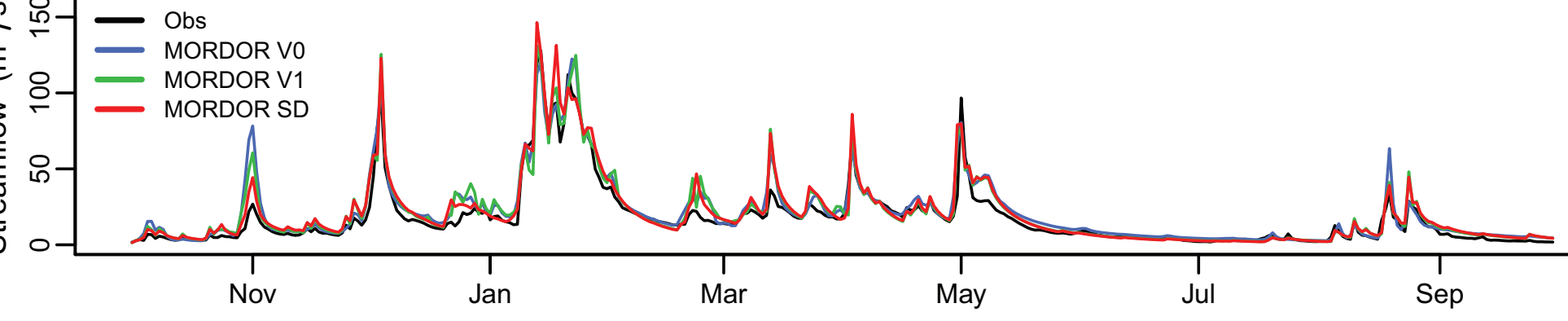
Souloise@Infernet

Year 1992
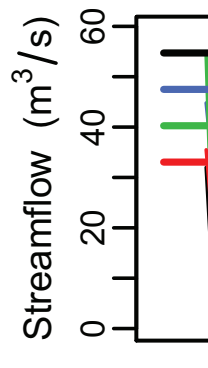

Obs

MORDOR V0

MORDOR V1

MORDOR SD

1

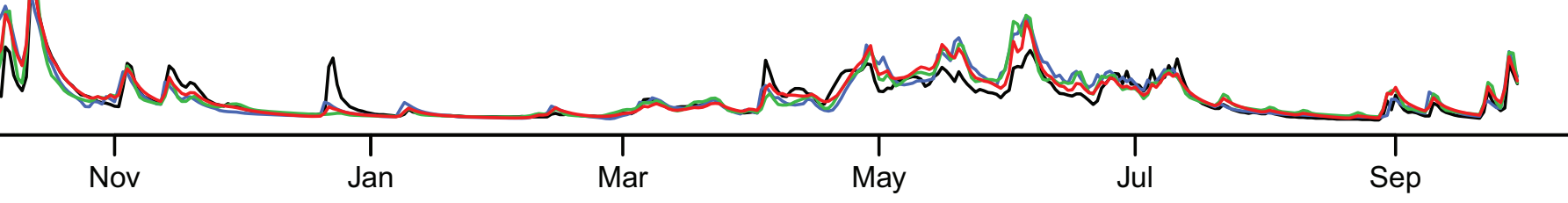

Year 1993
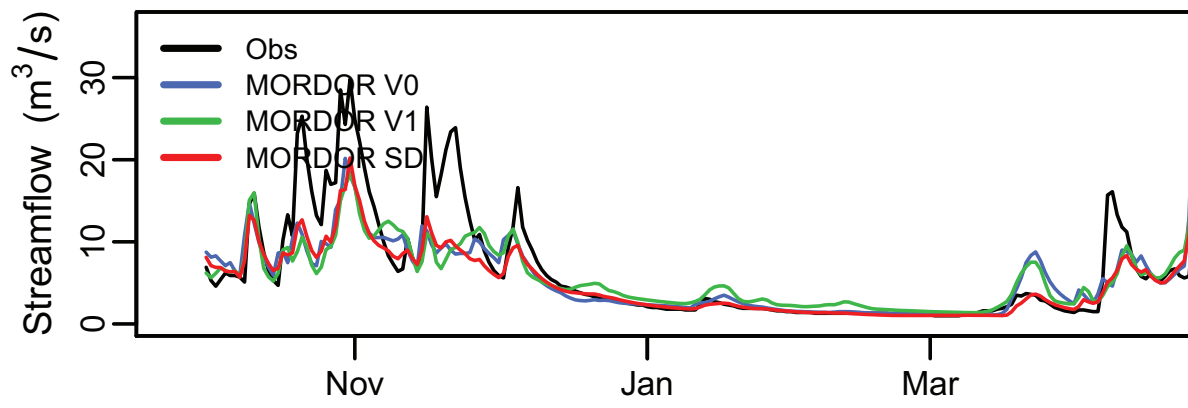

Year 1994

$\frac{\pi}{m}$
m
$\xi$
3
0
0
0
0
0

$-$

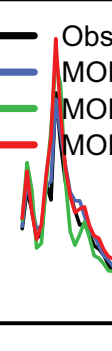

MOR

RDOR SD

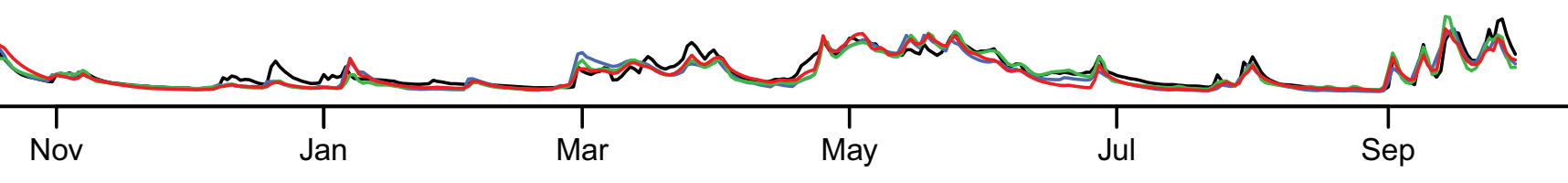

Year 1995

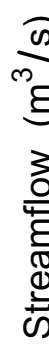

- Obs

$-\begin{aligned} & - \text { MORDOR V0 } \\ & \text { MORDORIV1 }\end{aligned}$

- MORDOR SD

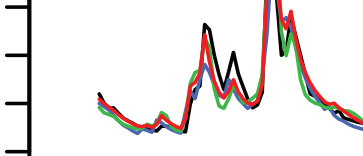

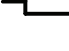

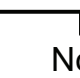

Nov
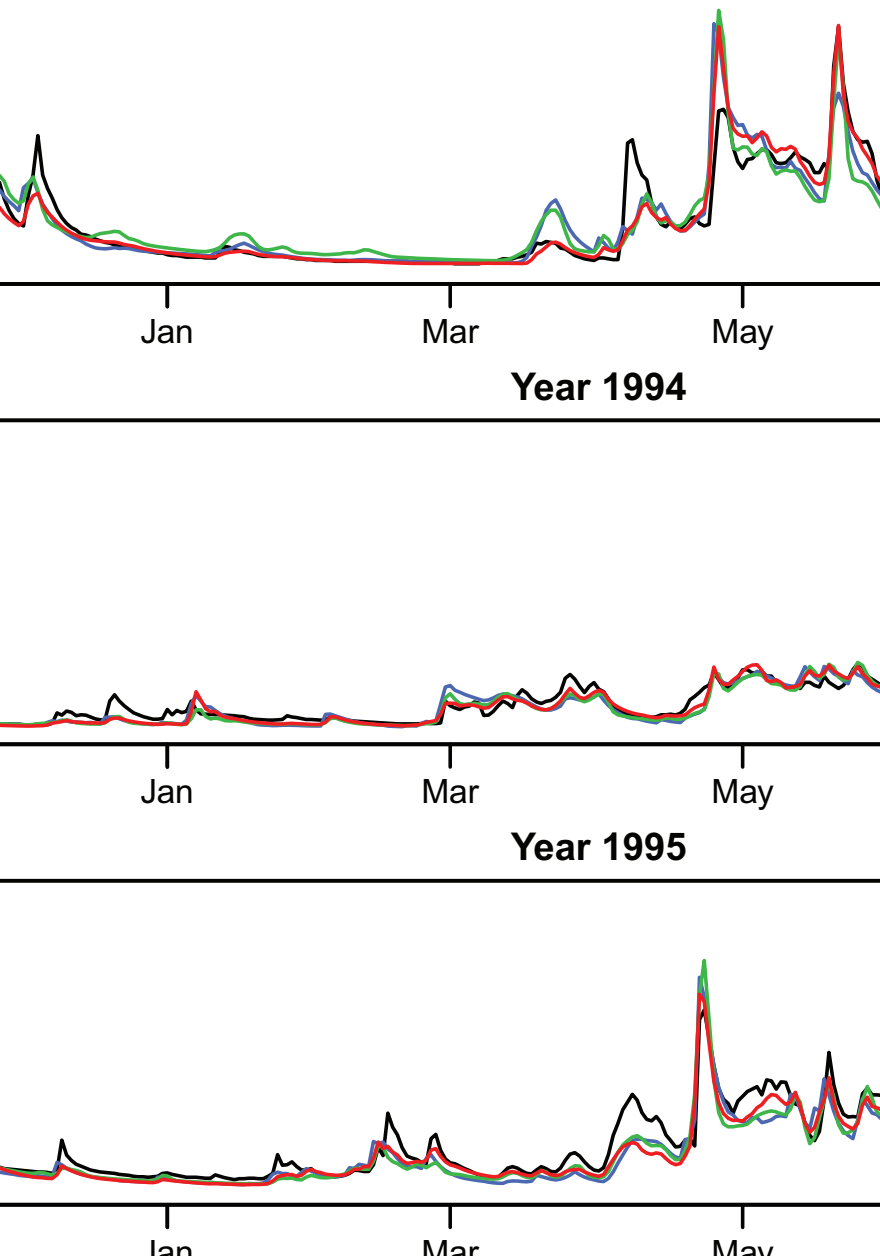

May
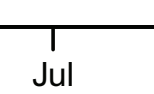

ep 
Souloise@Infernet

Year 2004
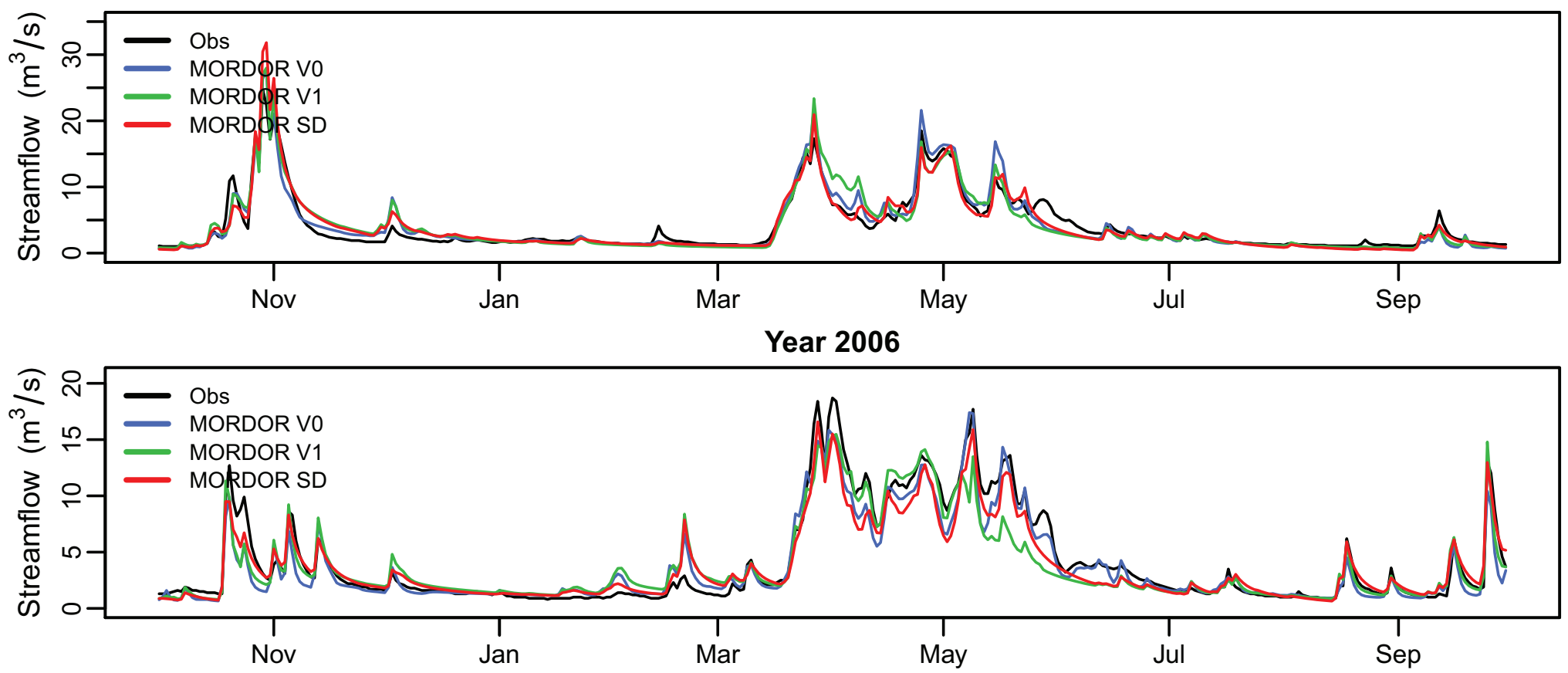
Tarn@Cocures

Year 1986
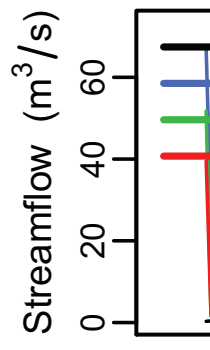

Obs

MORDOR V0

MORDOR V1

MORDOR SD
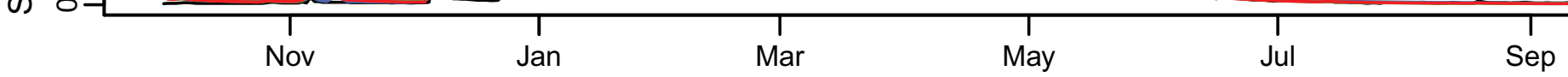

Year 1987

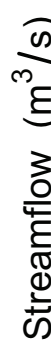

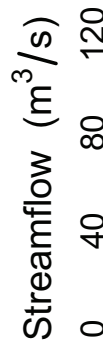
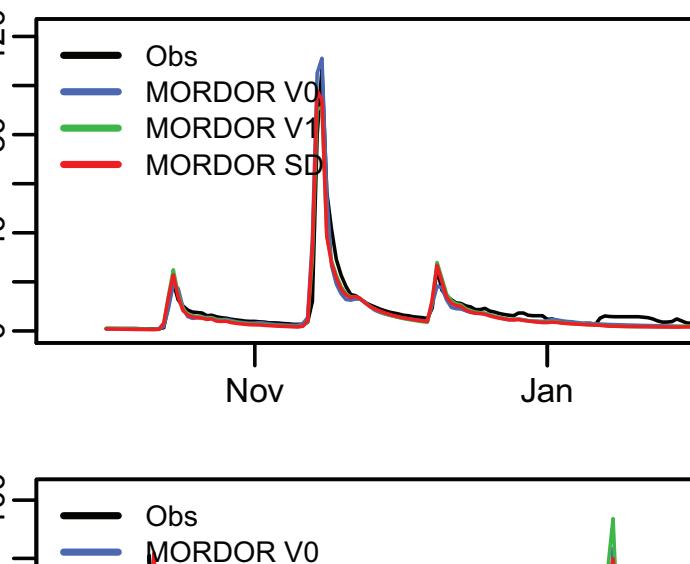

OR V0

-1 MORDOR V1

-1 MORDOR SD

है

sis
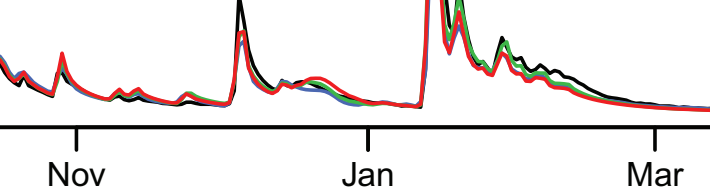

anan

Year 1988

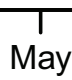

Jul

Sep

Year 1988
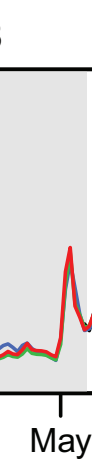

Year 1989
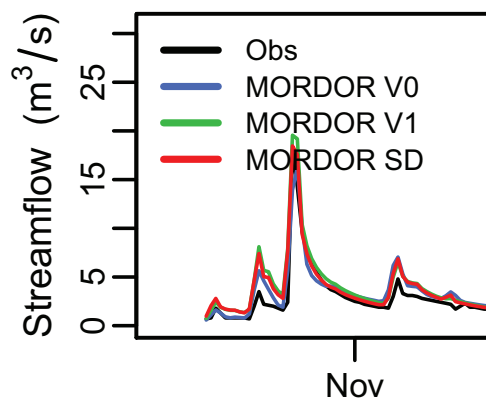

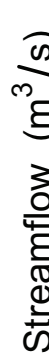

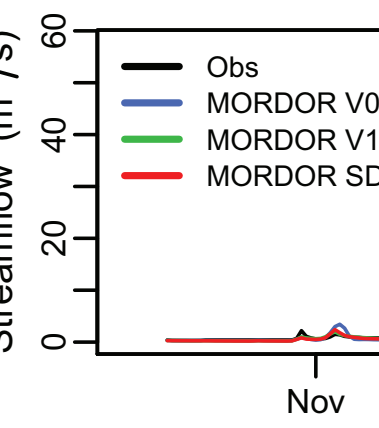

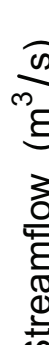

은-1

Obs

- MORDOR V0

- MORDOR V1

- MORDOR SD
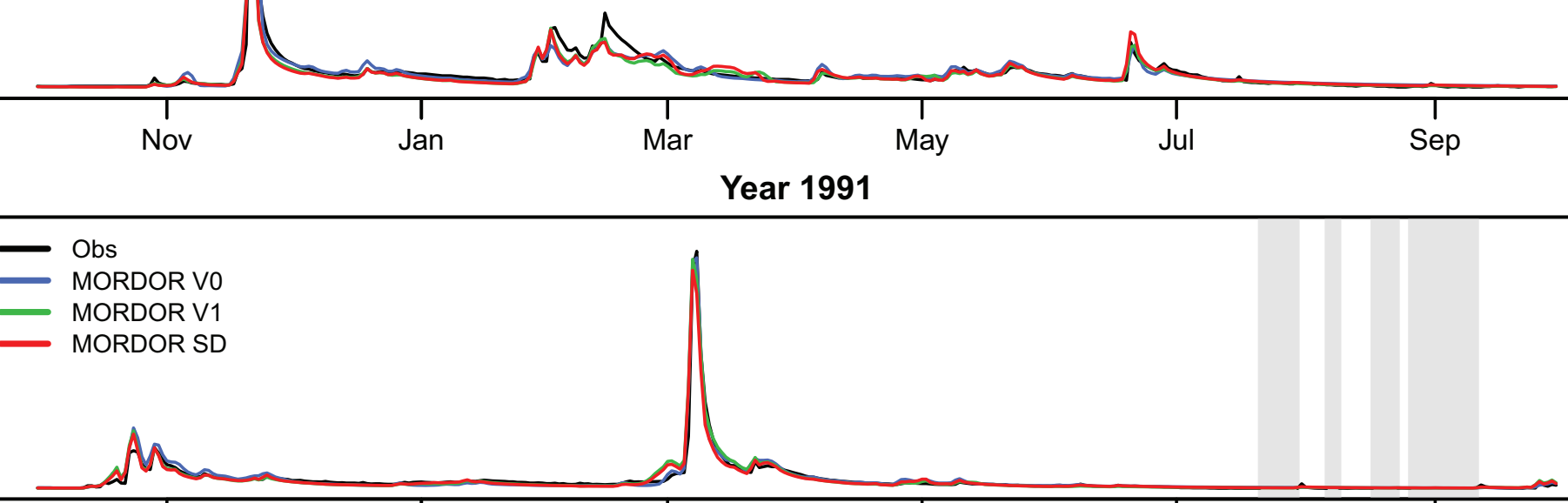

Year 1991 

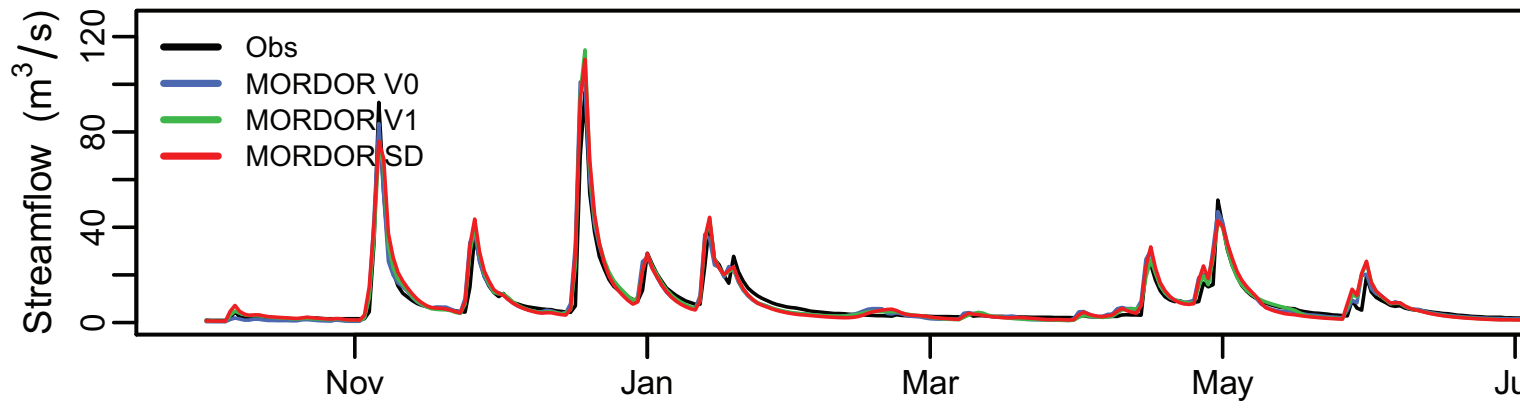

Year 1999
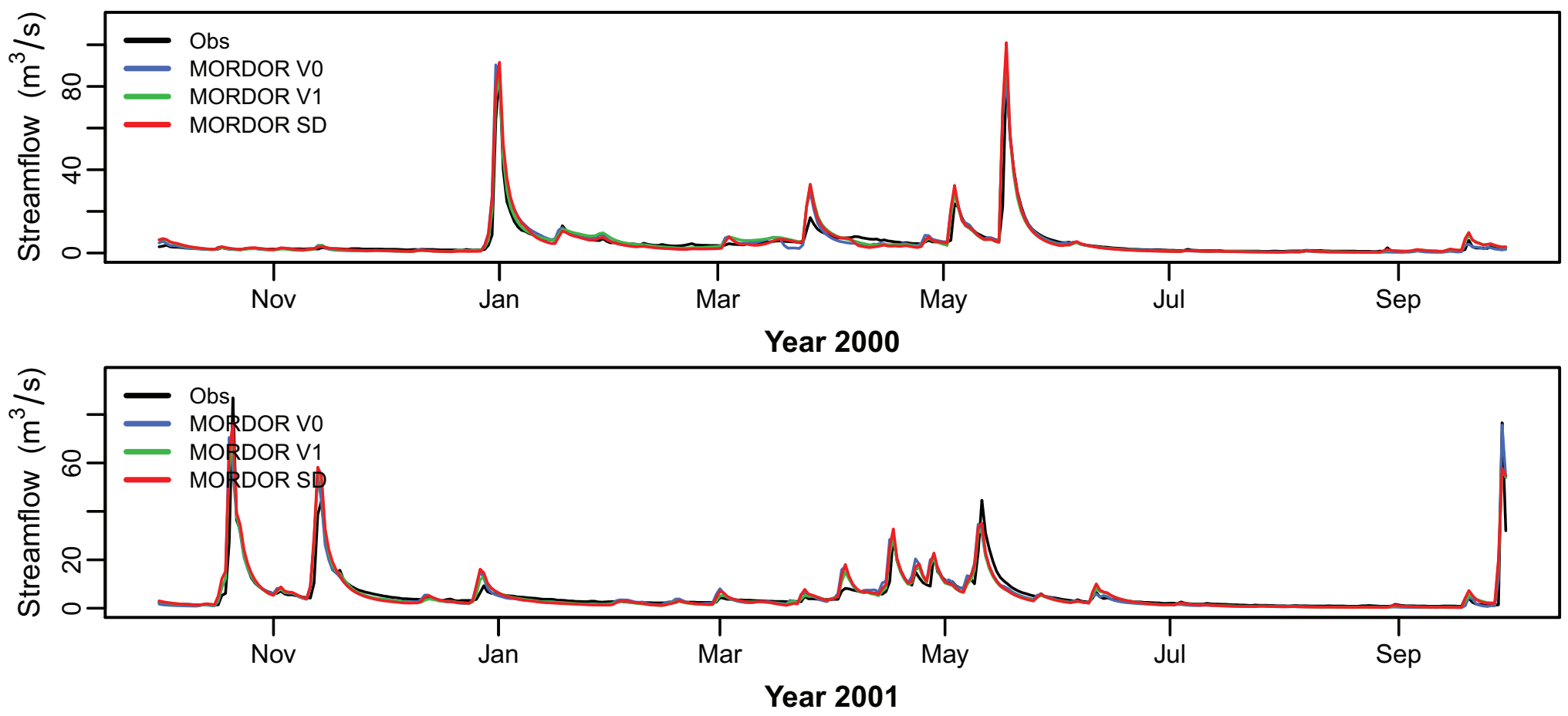

Jan

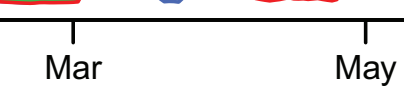

Year 2000
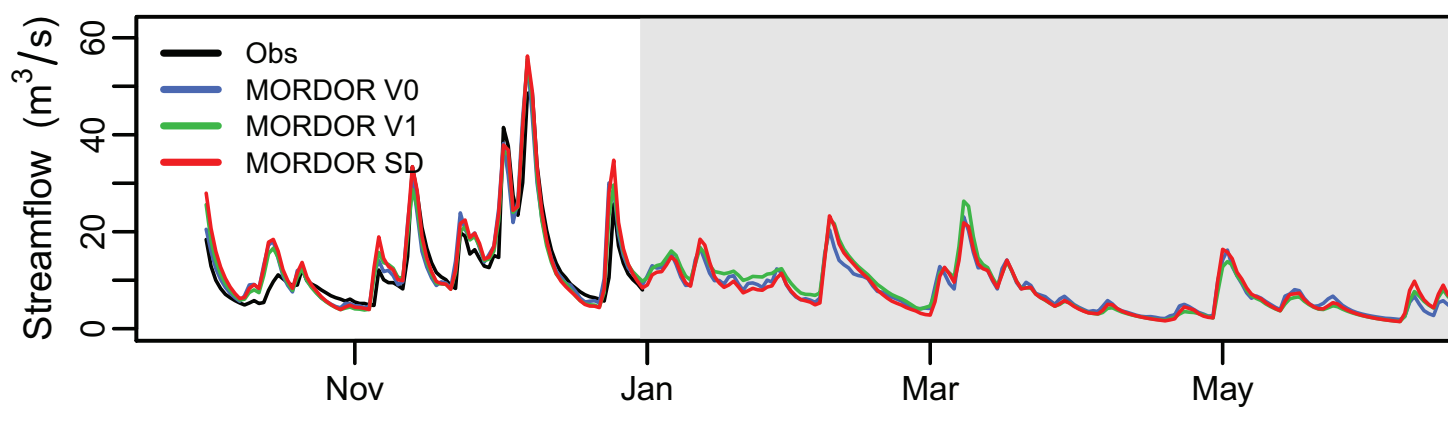

Year 2002
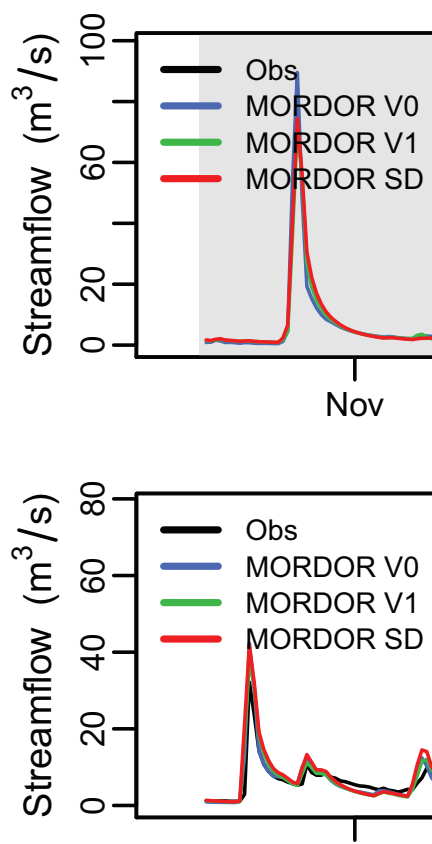

MORDOR V0
MORDOR V1

$$
\text { MORDOR V1 }
$$

- MORDOR SD

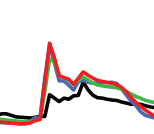


Tarn@Cocures

Year 2004
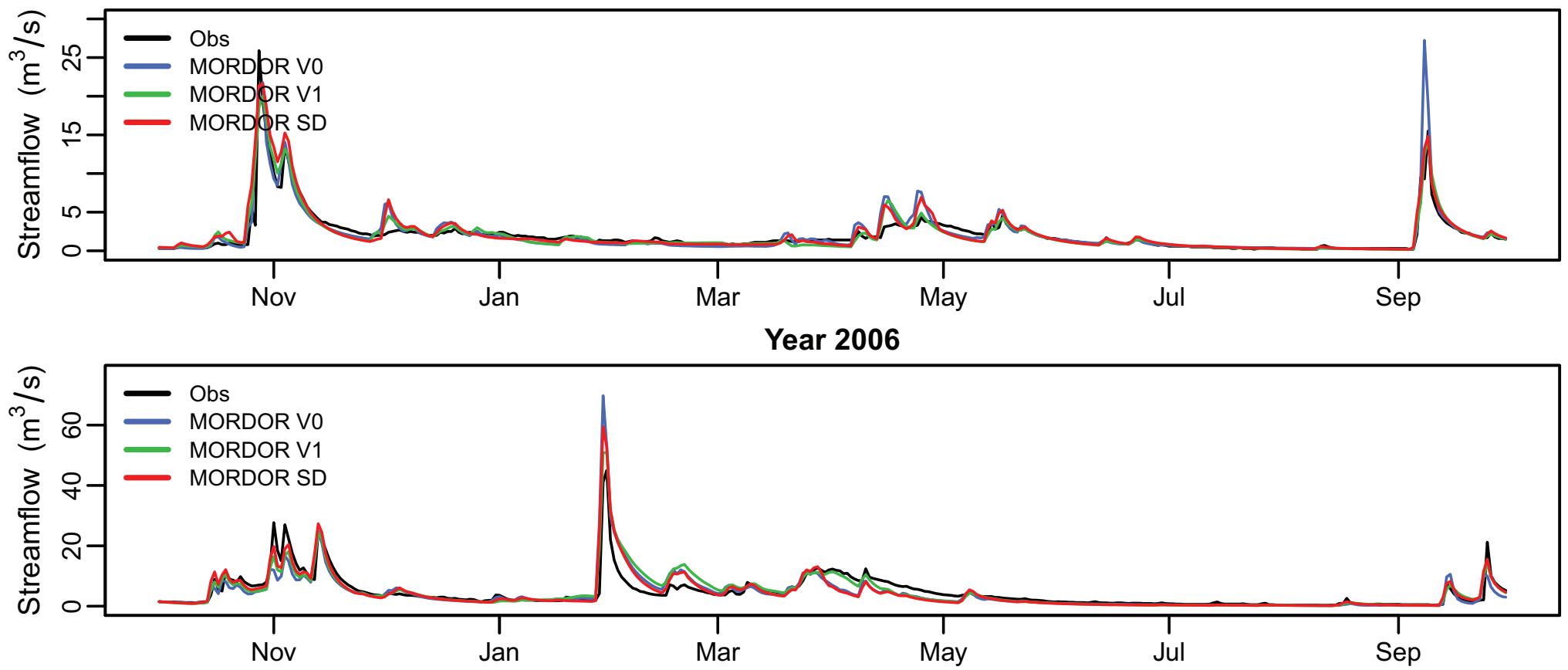


\section{Tarn@Millau}

Year 1993
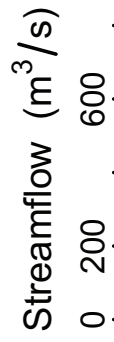

— MORDOR V0

- MORDOR V1

- MORDOR SD

-

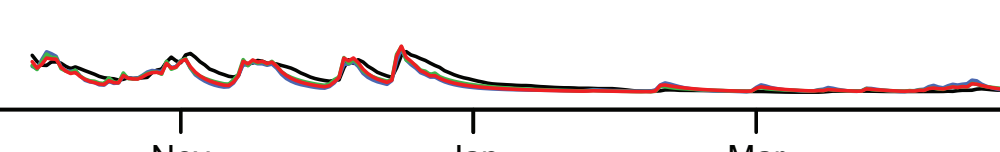

Year 1994

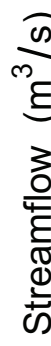

$\longrightarrow$ Obs

○- $=\begin{aligned} & \text { MORDOR V0 } \\ & \text { MORDOR V1 }\end{aligned}$

O- -7 MORDOR SD

(n)

$\circ-1$

$\rightarrow M$

Nov

Jan

Mar May

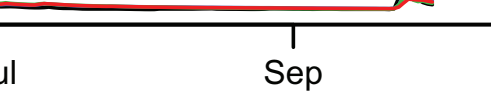


Tarn@Pinet

Year 1993
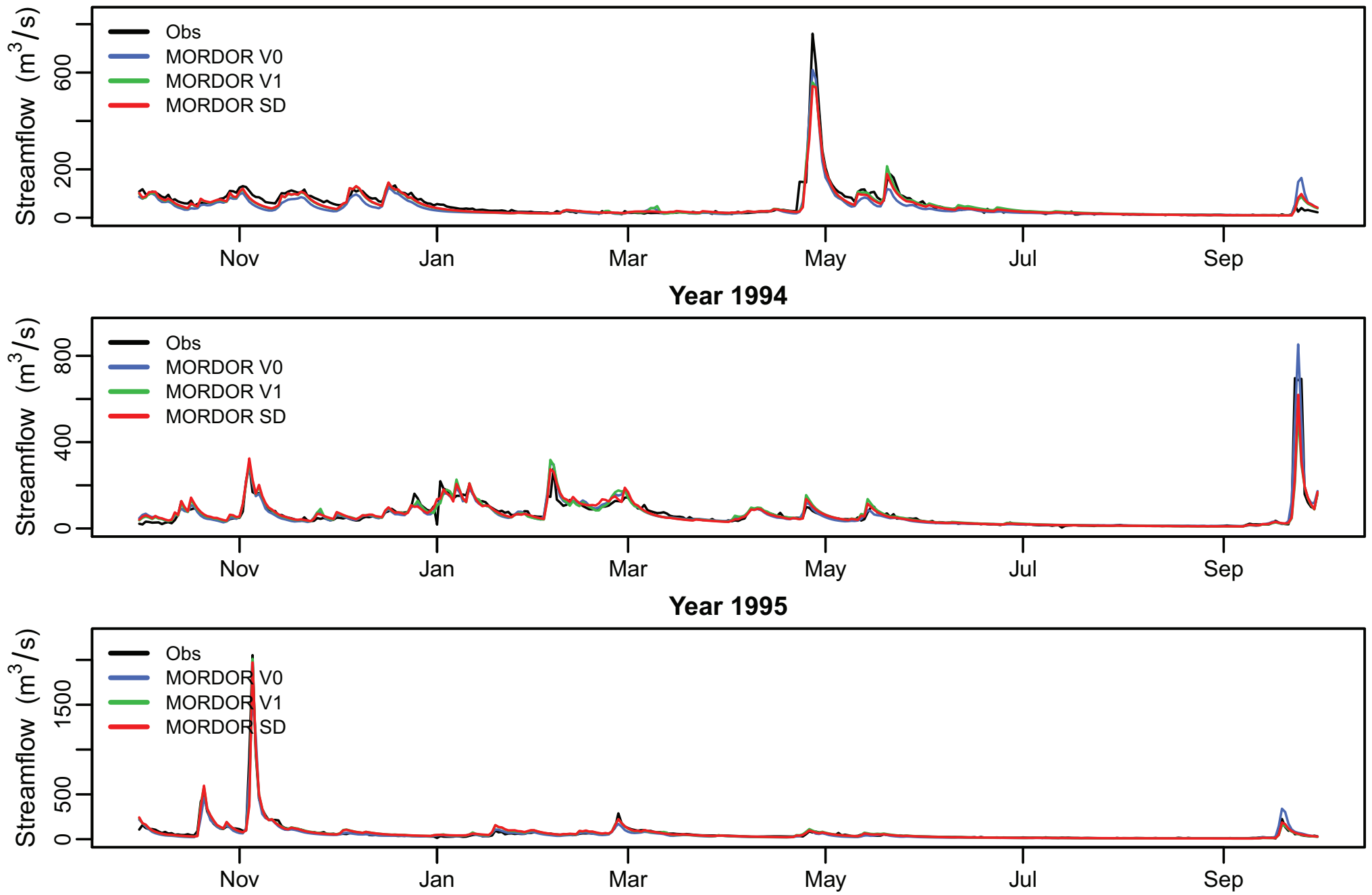
Taurion@RocheTalamie

Year 1964

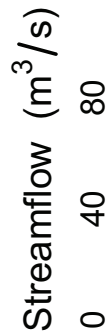

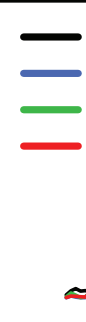

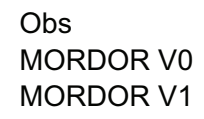

Year 1965

क

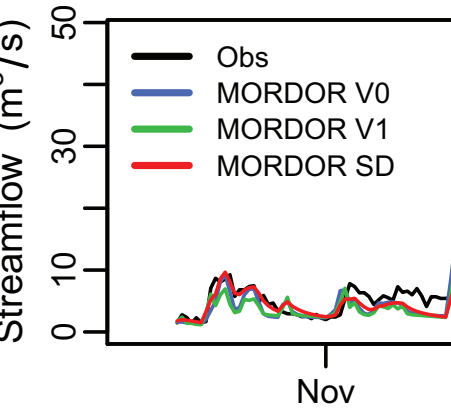

Antmand
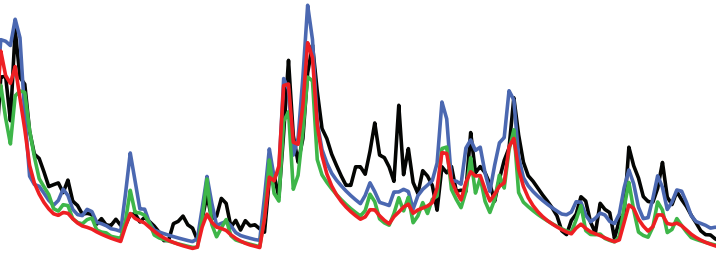

Mar May

Year 1966

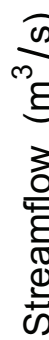
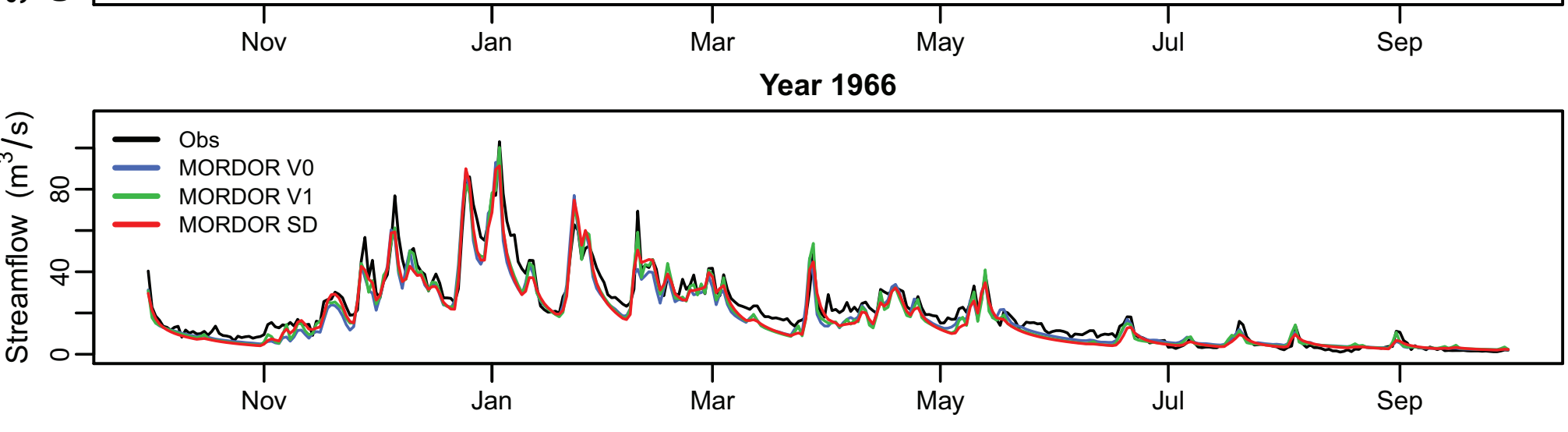

Year 1967

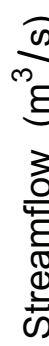

$\frac{0}{0}$
m
5
3
0
0
$\frac{1}{0}$
0
0
0
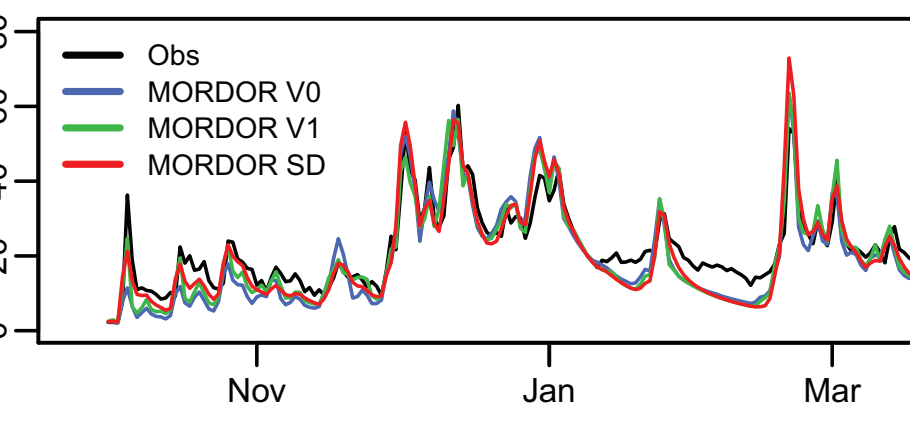

somadule

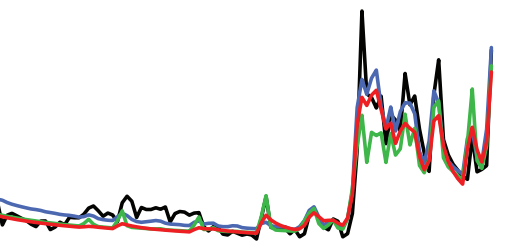

Jul Sep

Sep
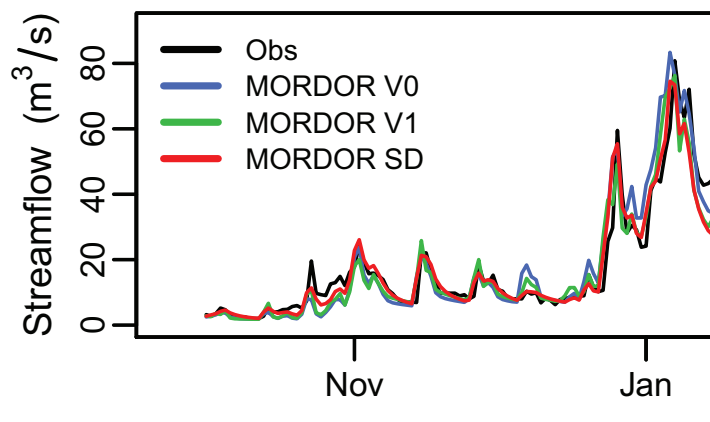

\section{Year 1968}

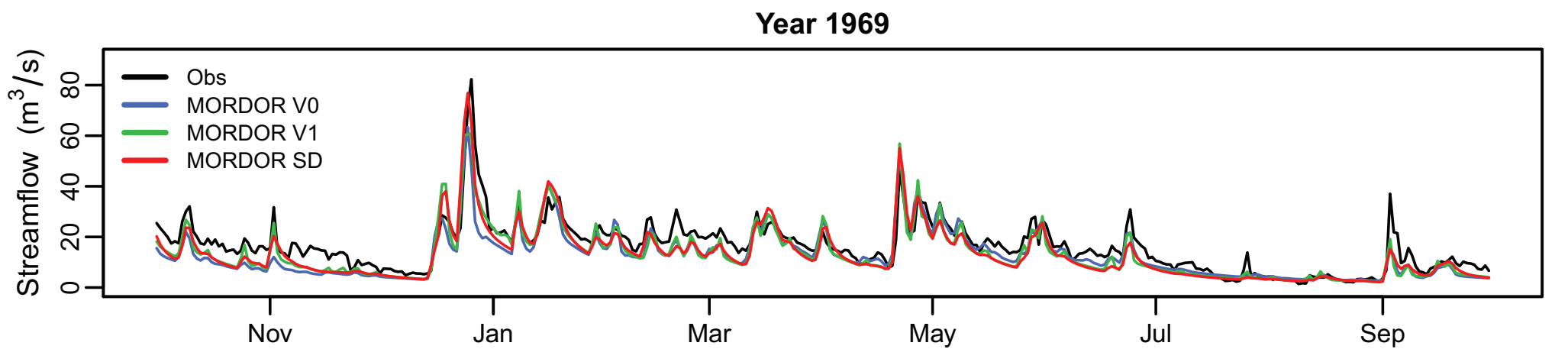




\section{Taurion@RocheTalamie}

\section{Year 1976}
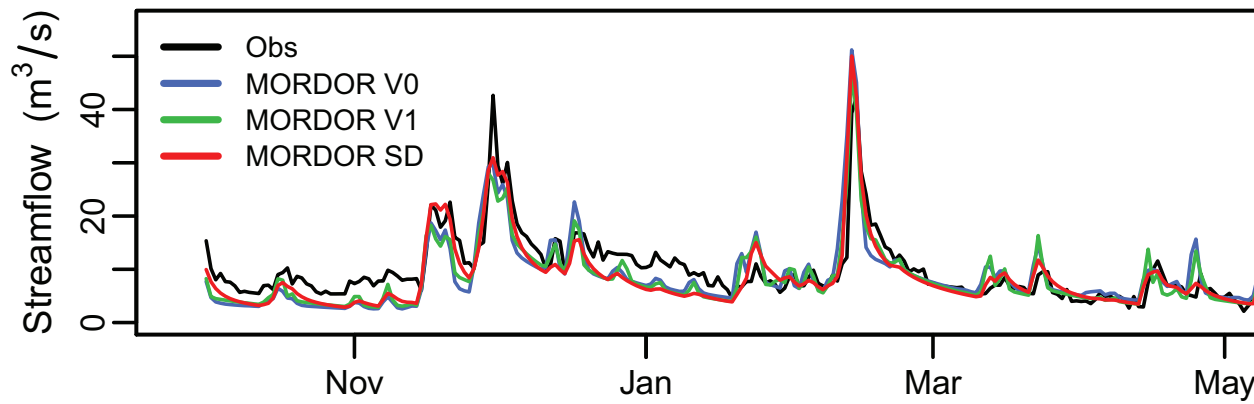

Year 1977
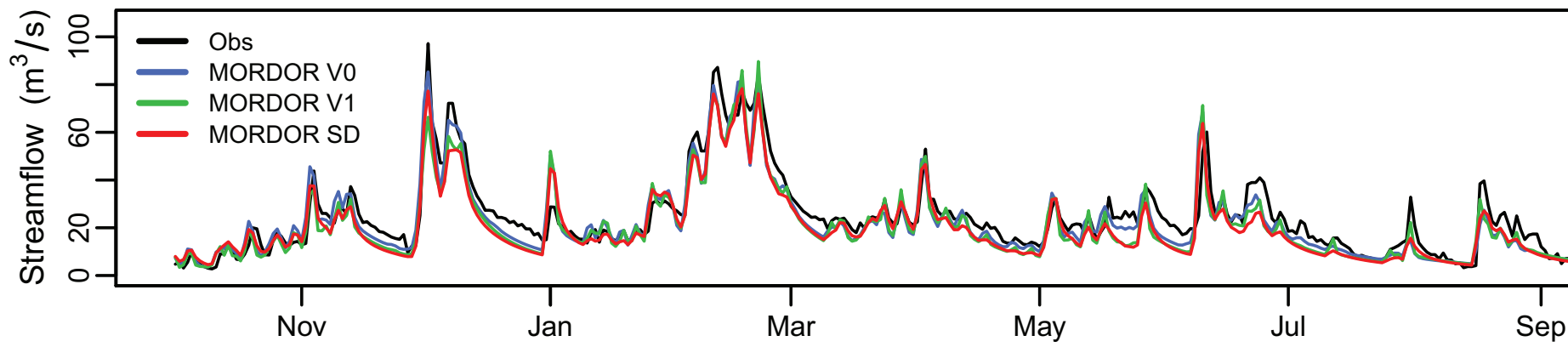

Year 1978

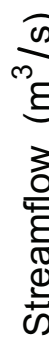

\section{- Obs}

- MORDOR VO

है $8-\left[\begin{array}{l}\text { MORDOR V1 } \\ \text { ( MORDOR SD }\end{array}\right.$

है $8-\left[\begin{array}{l}\text { MORDOR V1 } \\ \text { ह MORDOR SD }\end{array}\right.$
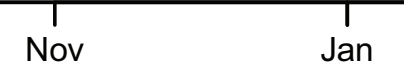

Mar

Year 1979
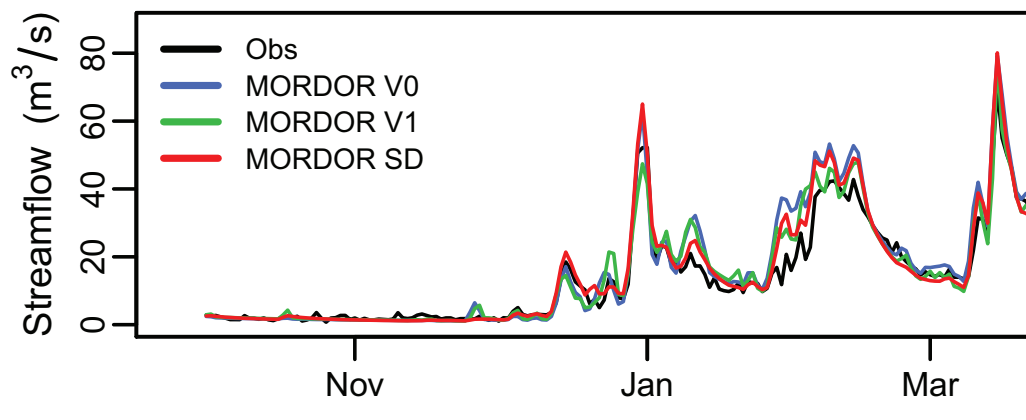

.
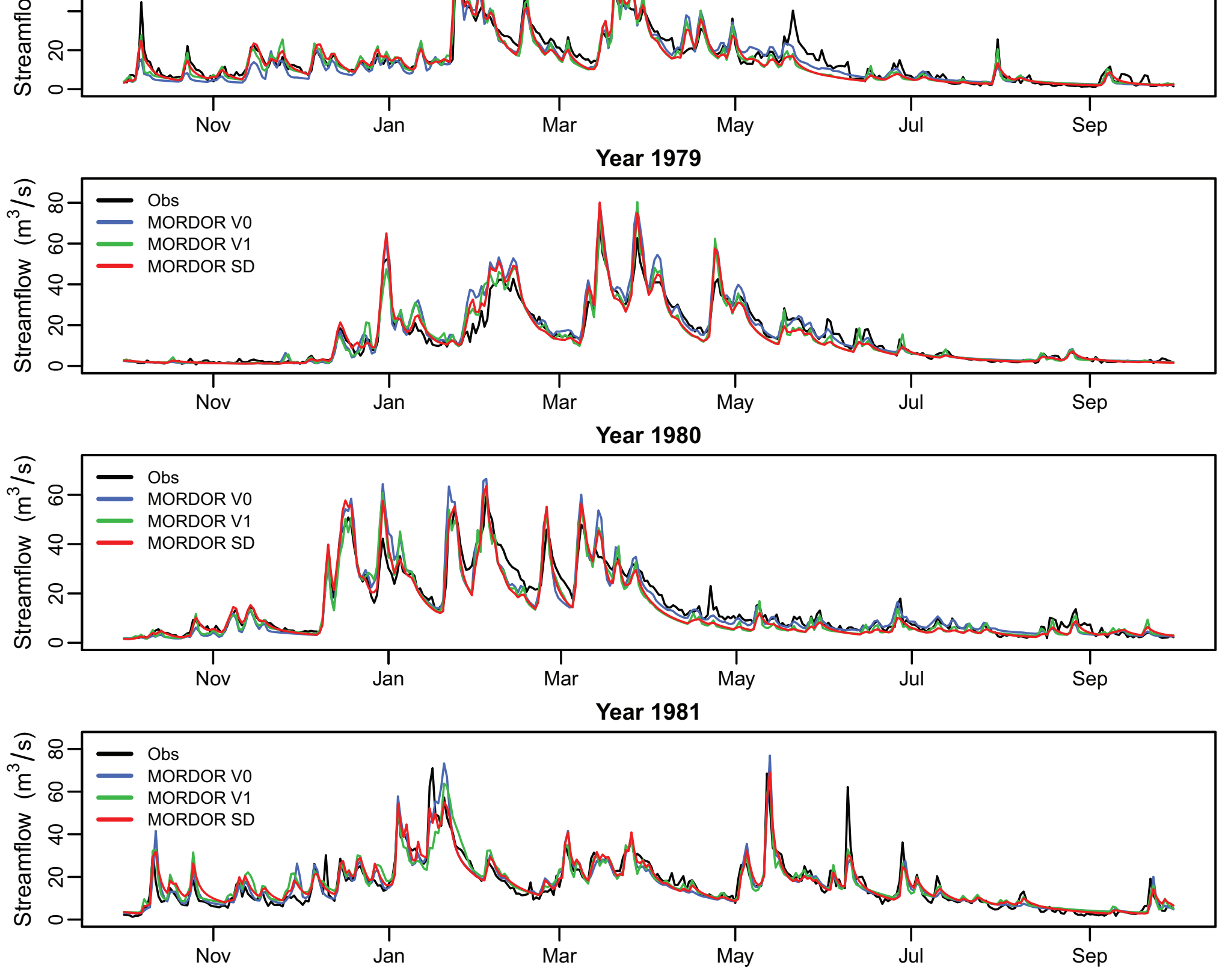


\section{Taurion@RocheTalamie}

Year 1982
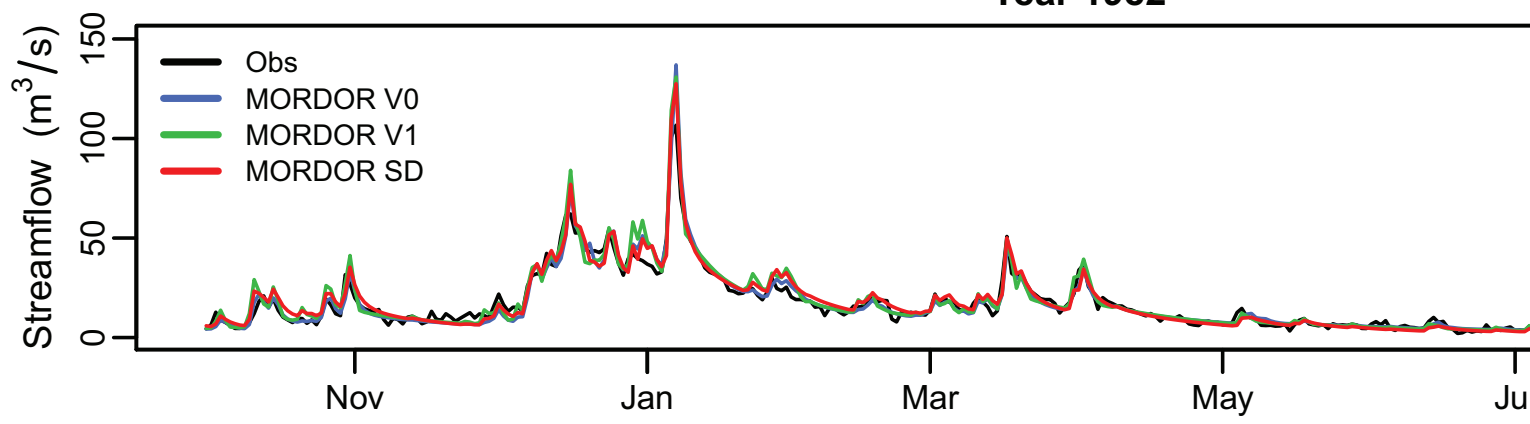

Year 1983
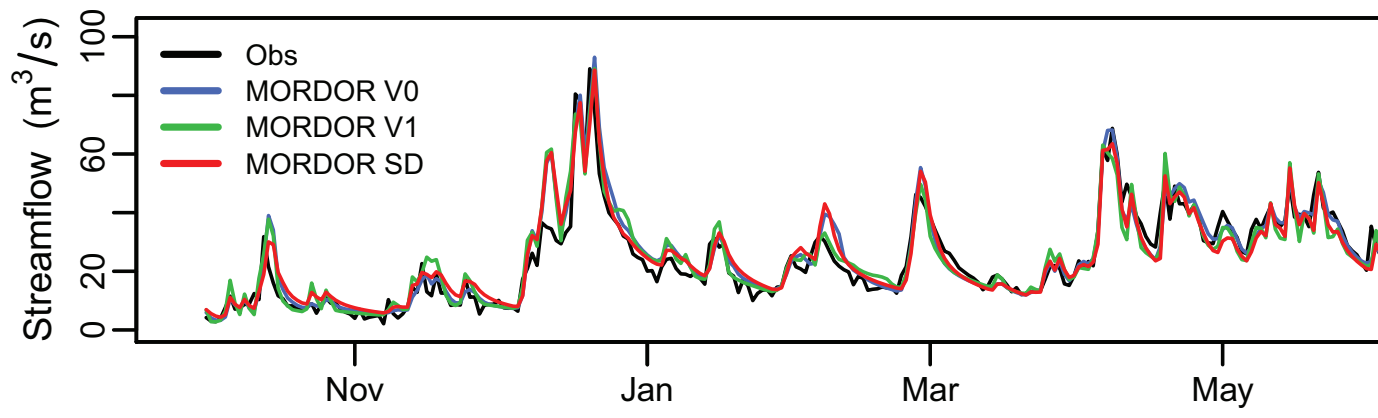

Year 1984
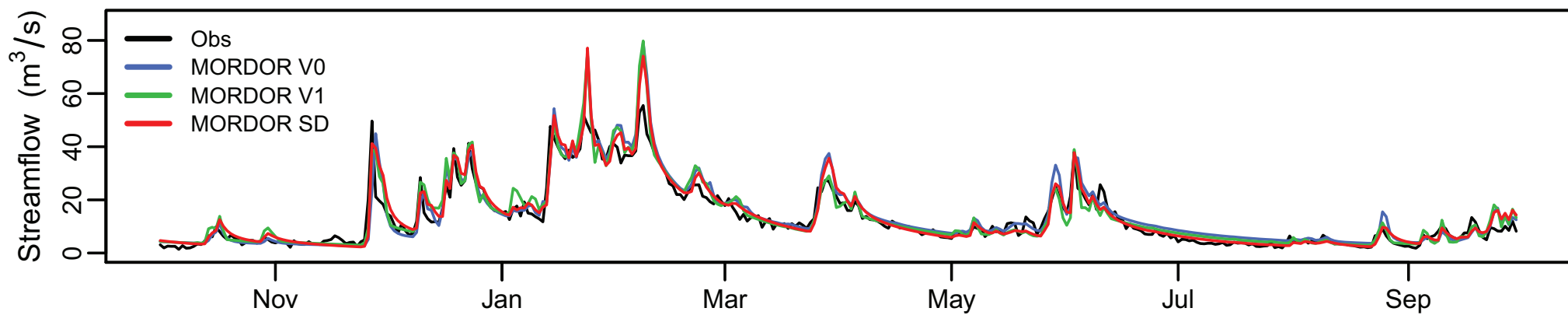

Year 1985
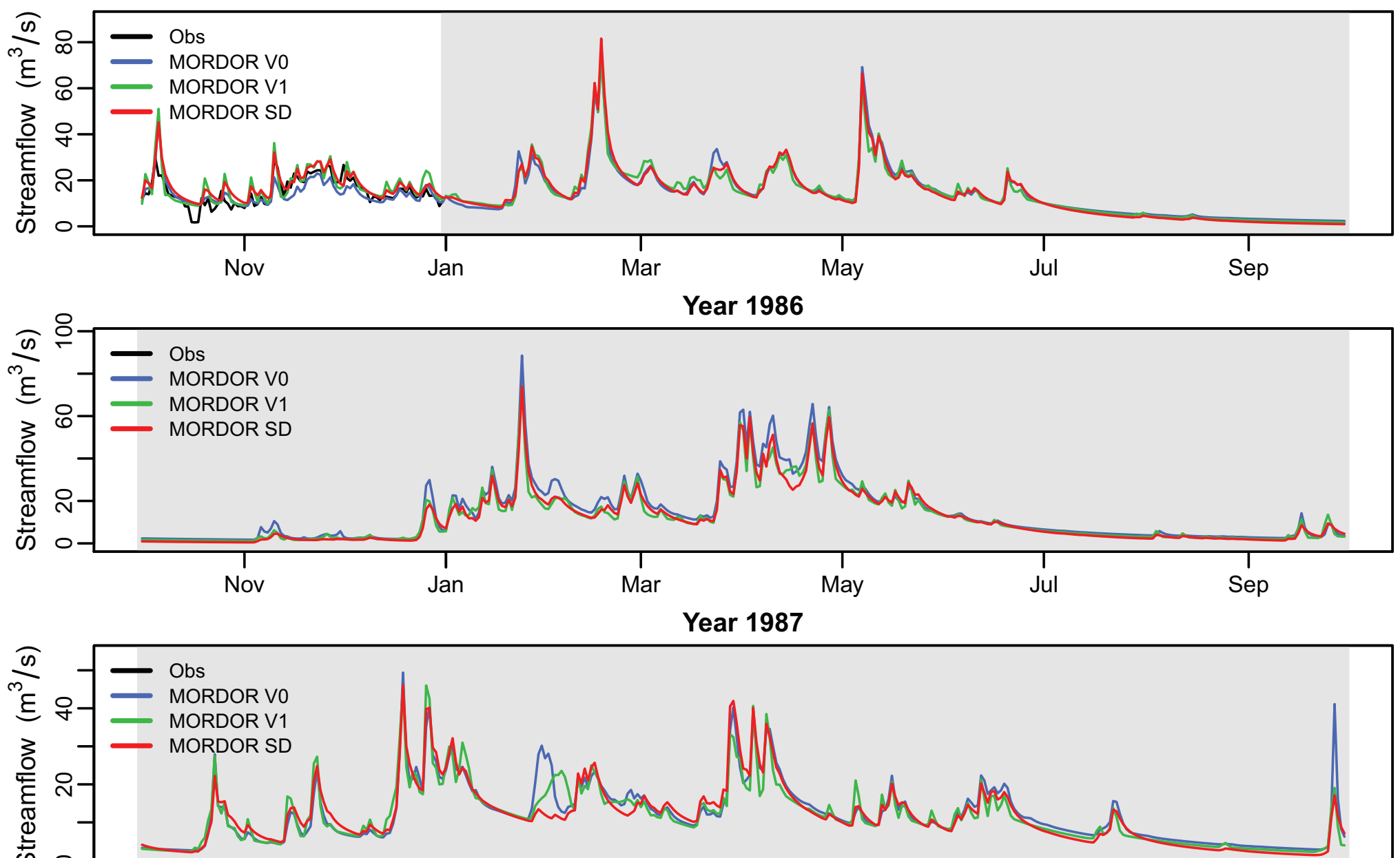

Year 1986

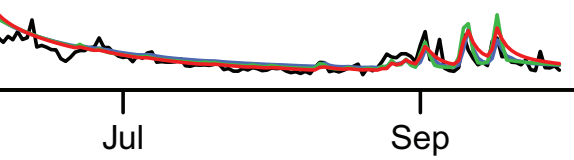




\section{Taurion@RocheTalamie}

\section{Year 1994}
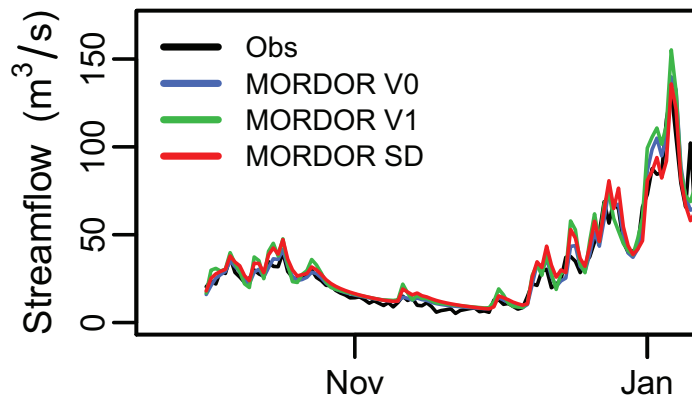

Lasumentents

Jan

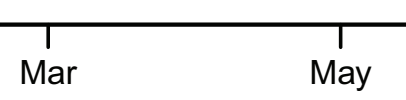

Year 1995
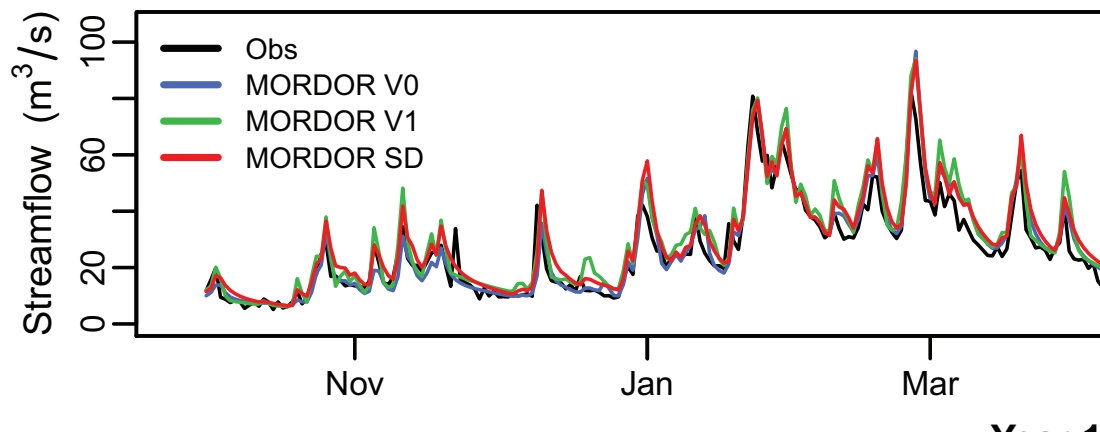

Year 1996

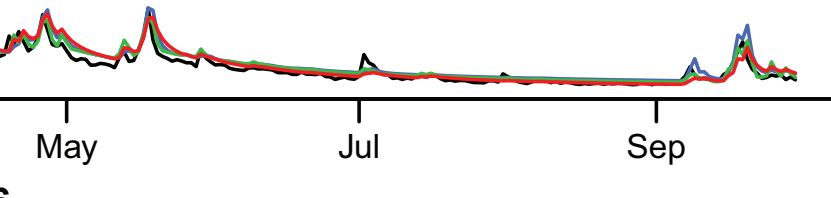

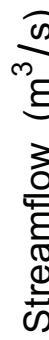

Obs
- MORDOR V0
MORDOR V1

으 MORDOR SD

等

Conasos Nov
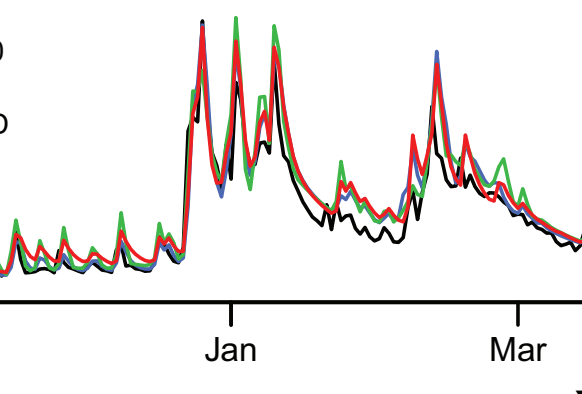

Ma

Year 1997
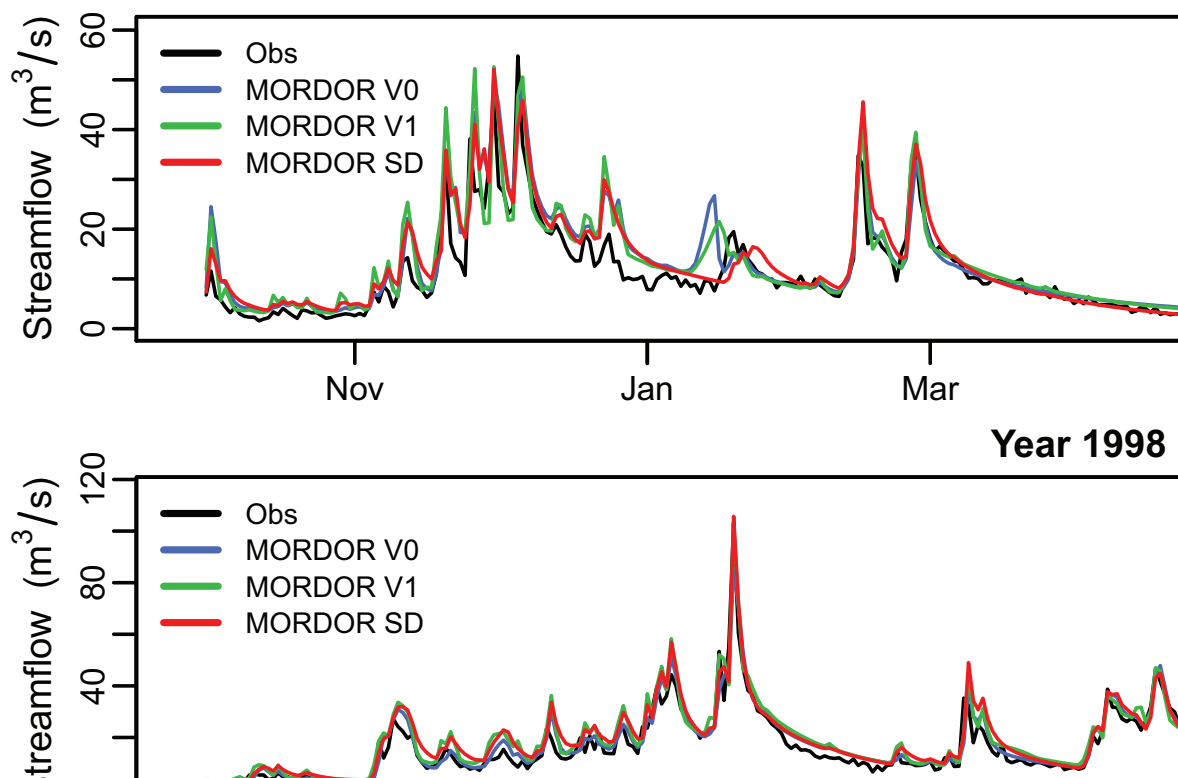

Year 1998
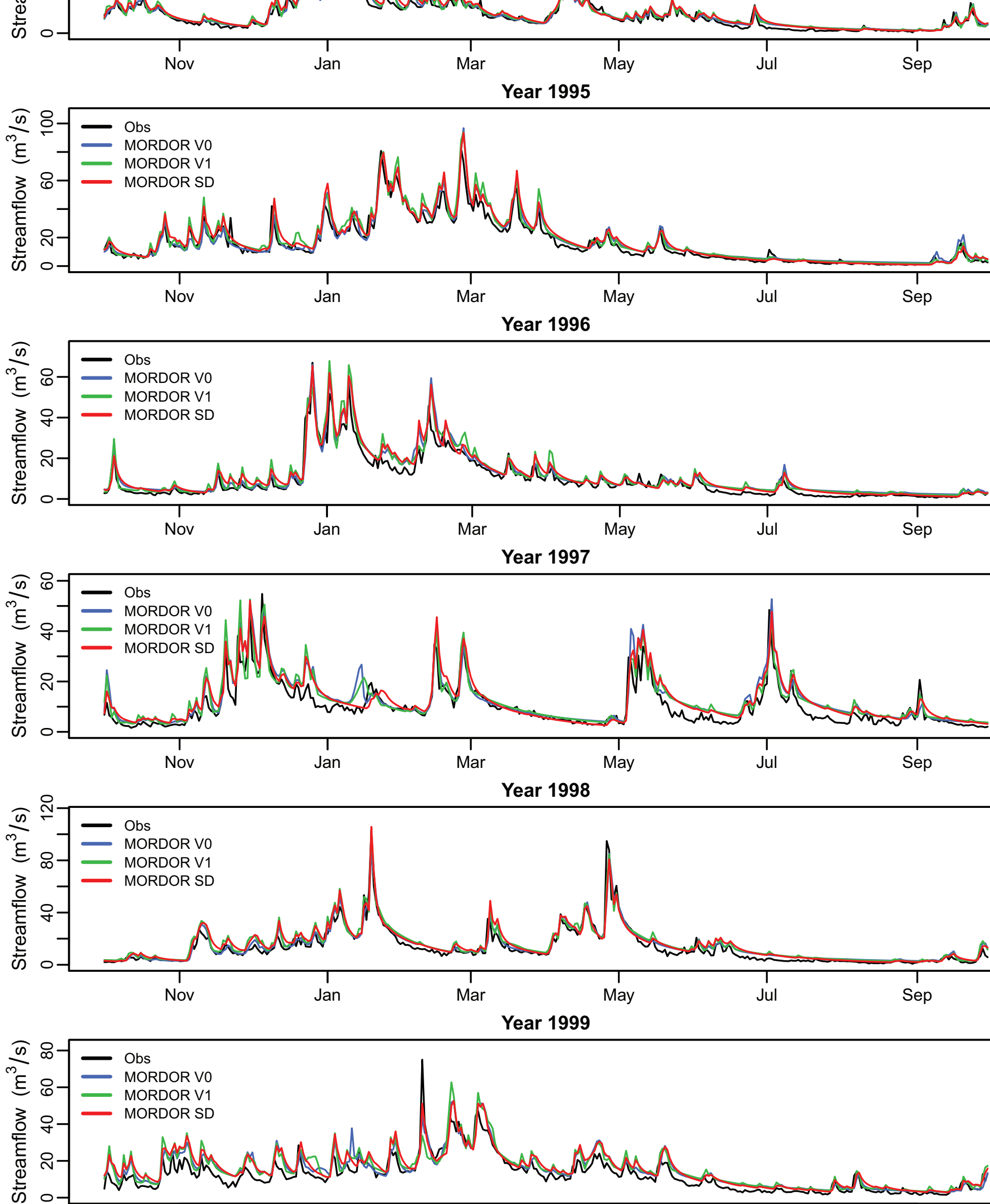


\section{Tet@Vinca}

Year 1995
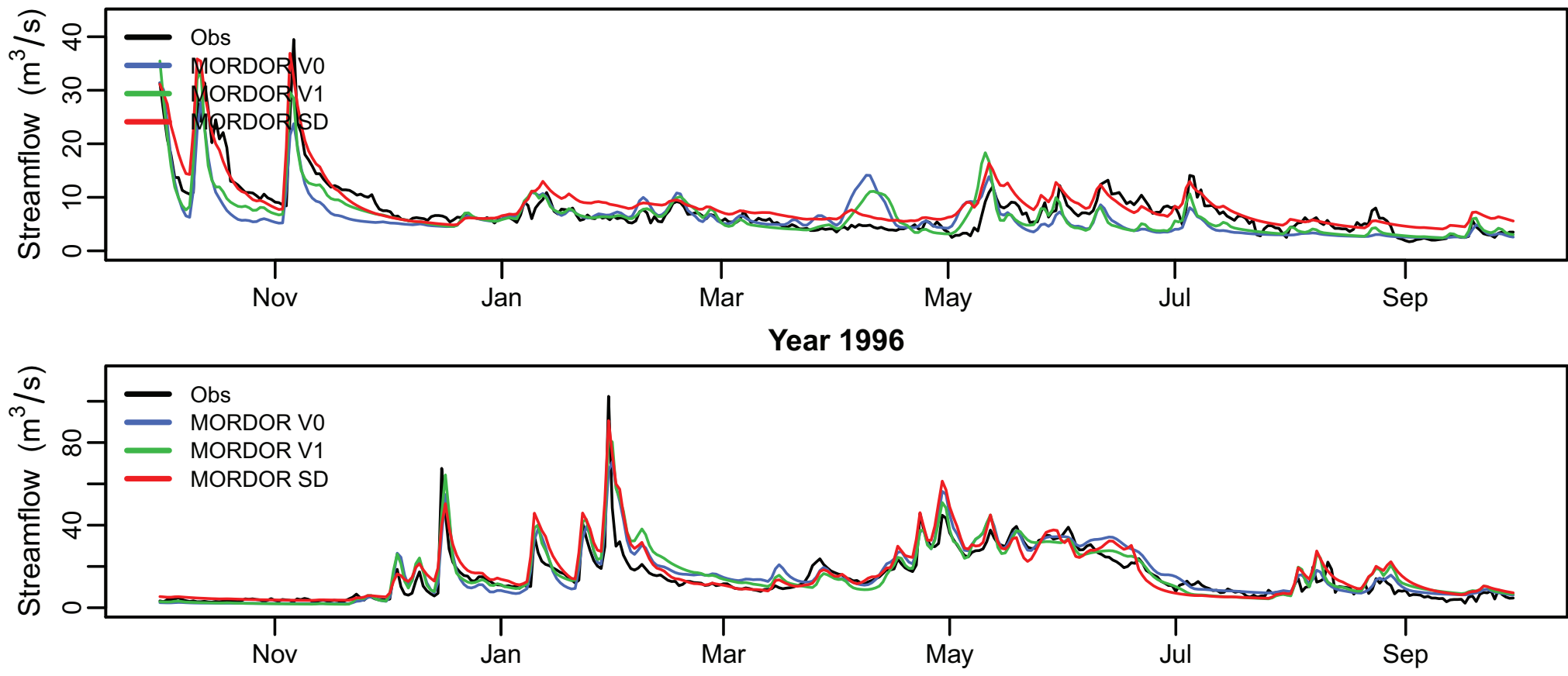

Year 1996
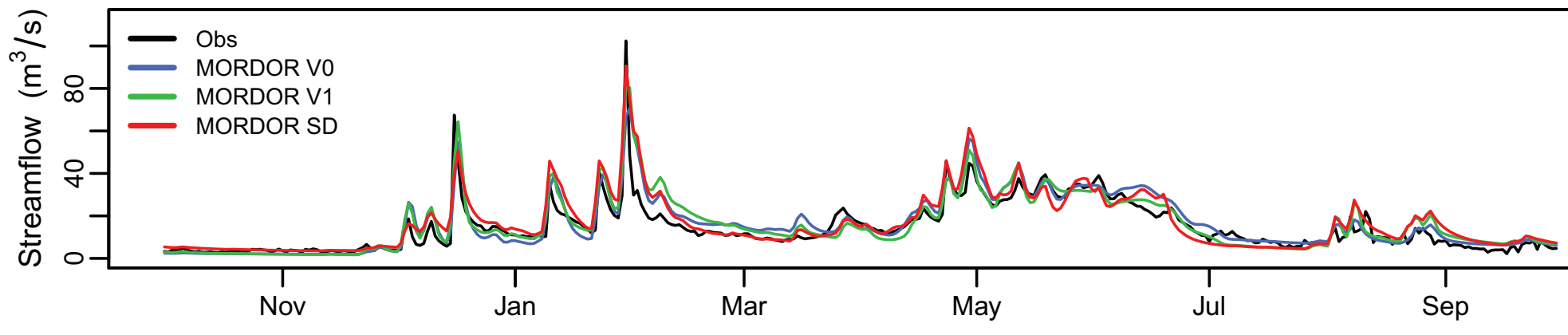

Year 1997
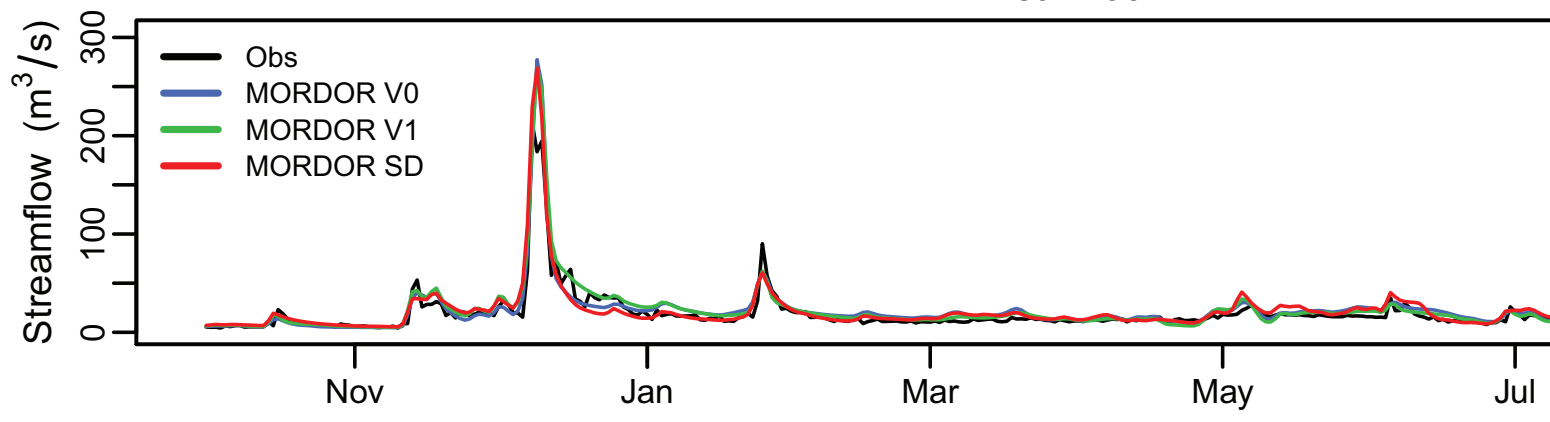

Sep

Year 1998
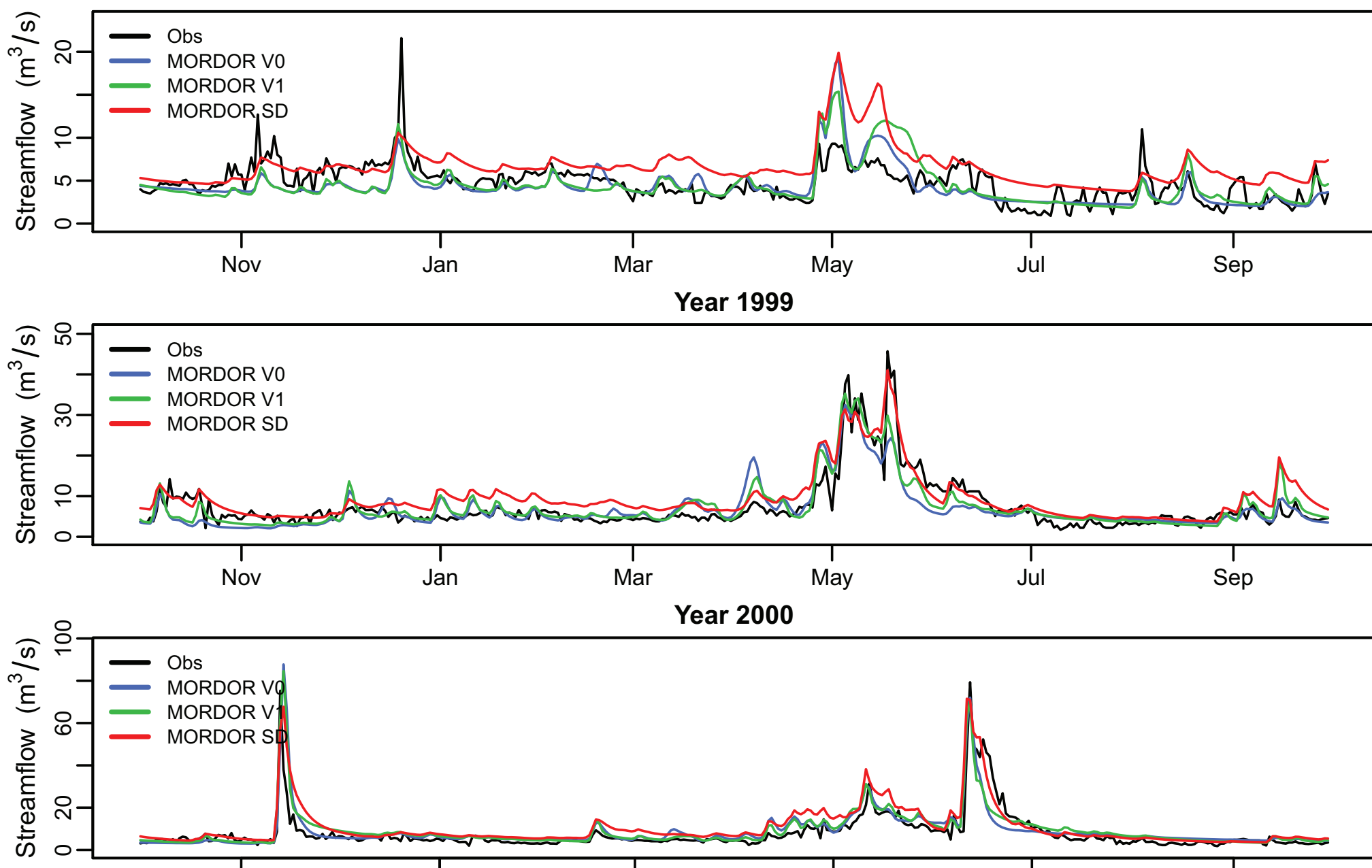

Obs

- MORDOR V0

- MORDOR V1

- MORDOR SD

$-12$
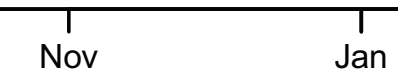

Mar

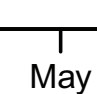

Year 2000

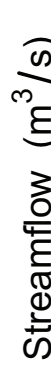

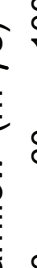

.

- Obs

- MORDOR V

$\longrightarrow$ MORDOR S

ㅇ-

○- 


\section{Tet@Vinca}

Year 2001
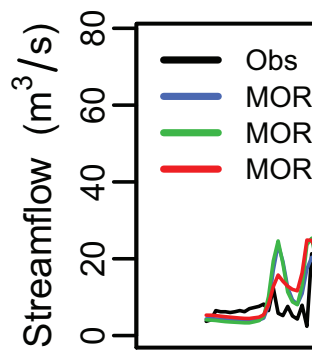

MORDOR V0

MORDOR V1

- MORDOR SD

CMn

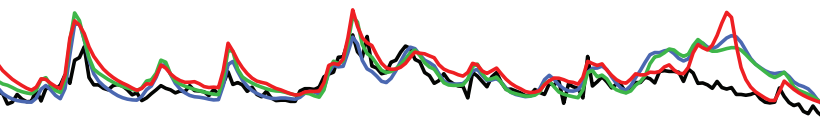

Nov Jan

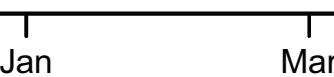

Mar May

Nonmans?

Year 2002
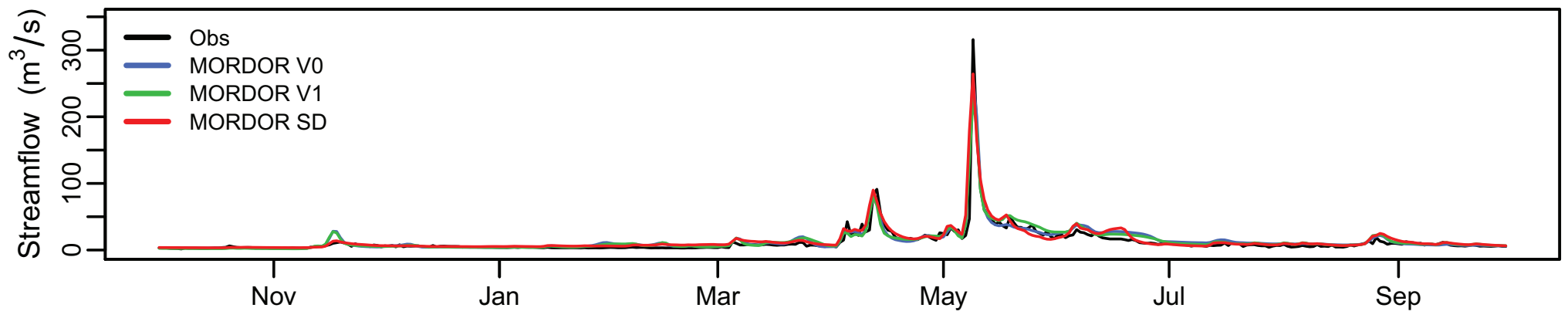

Year 2003

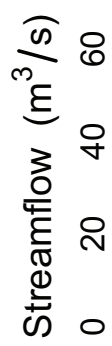

Obs

MORDOR V

MORDOR SD

Nov

Jan

Mar

May

Jul

Sep

Year 2004

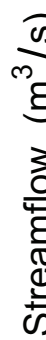

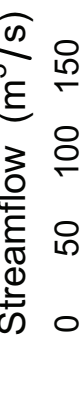

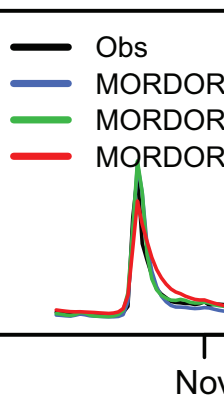

1
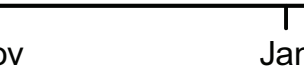

Jan

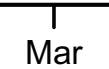

Mar

Year 2005
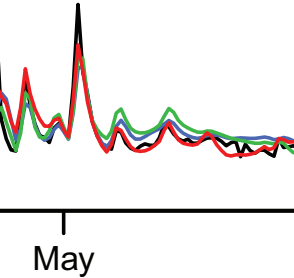

Jul

Sep

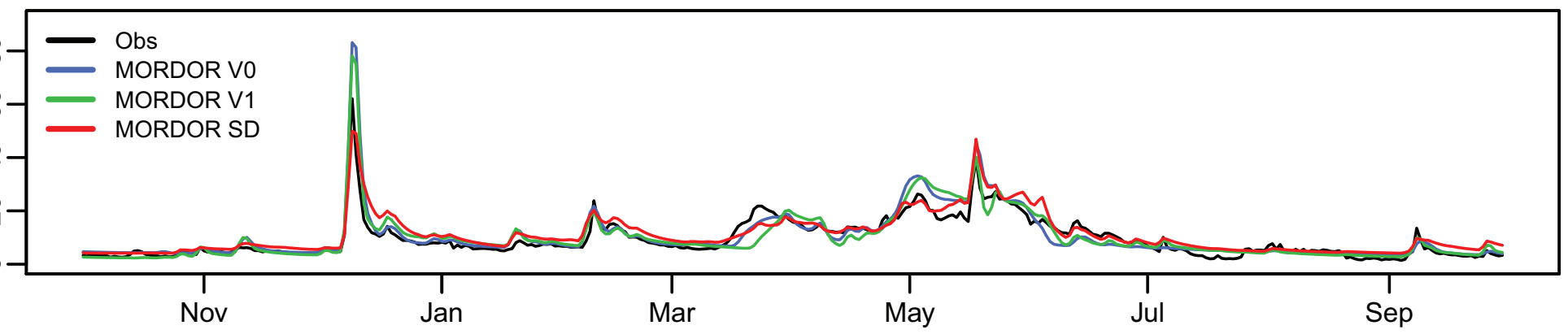

Year 2006

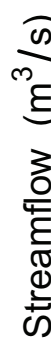

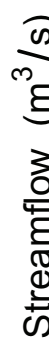

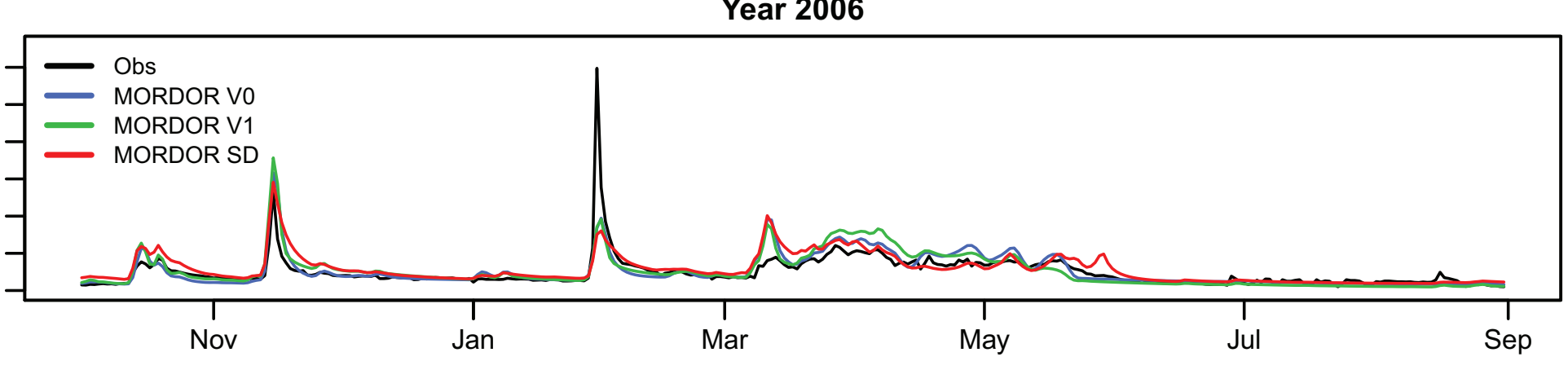




\section{Tinee@PontLune}

Year 1982

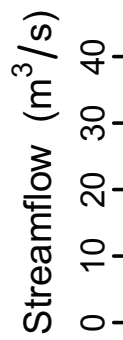
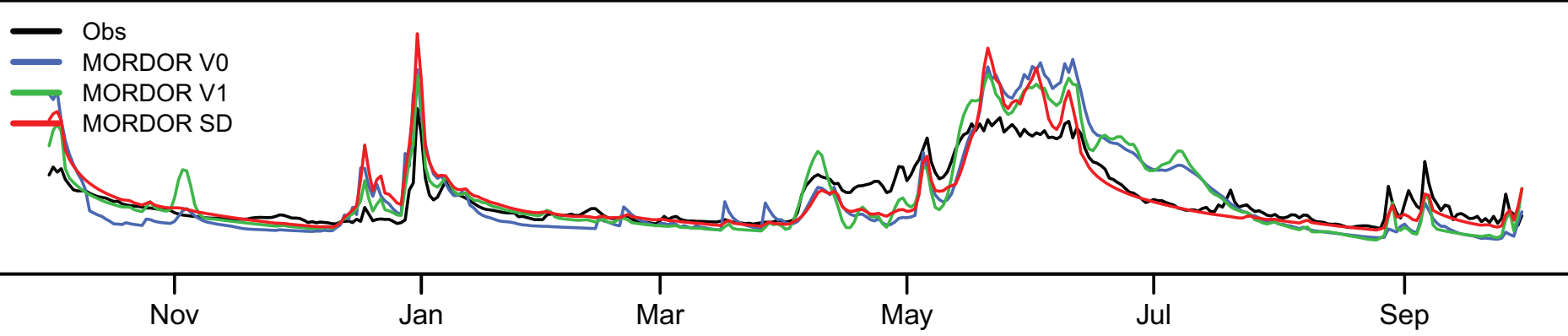

Year 1983
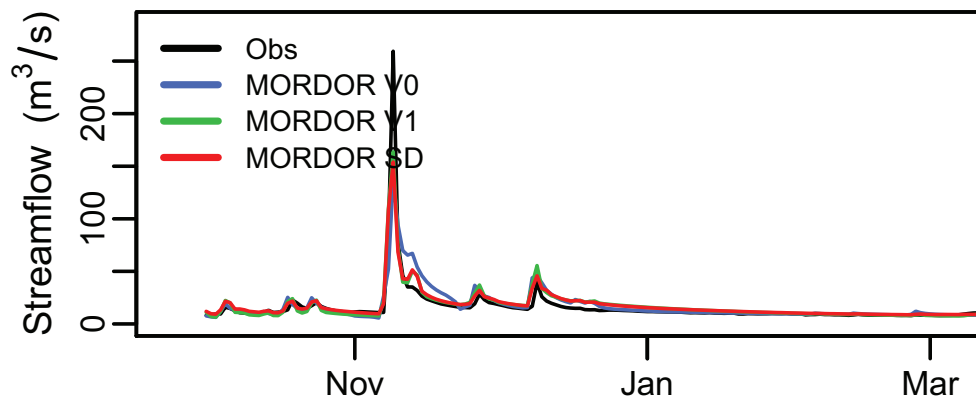

Year 1984
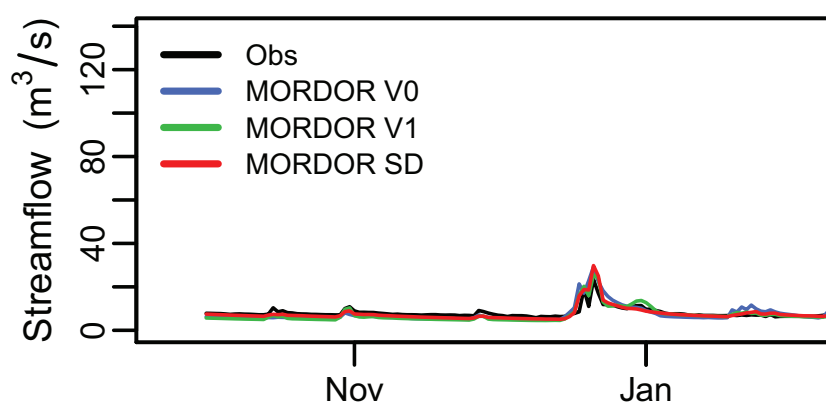

Jan

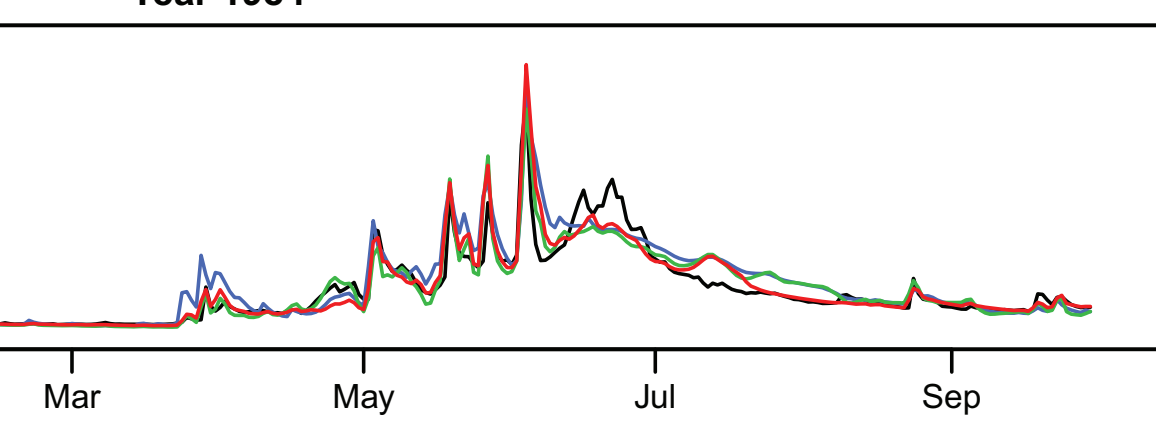

Year 1985
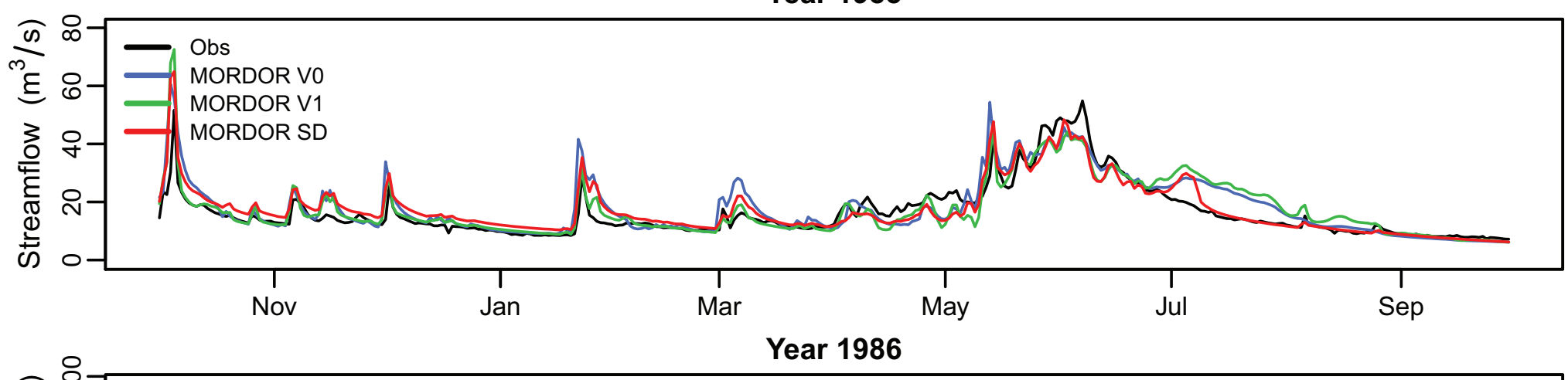

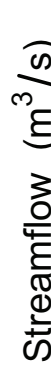
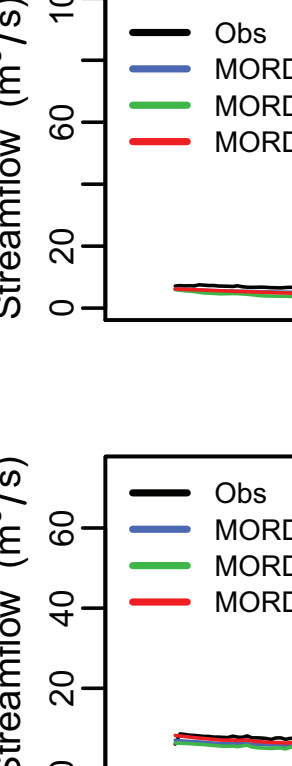

- - MORDOR VO

MORDOR V1

- MORDOR SD

ran

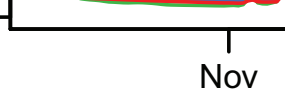

Nov

Jan

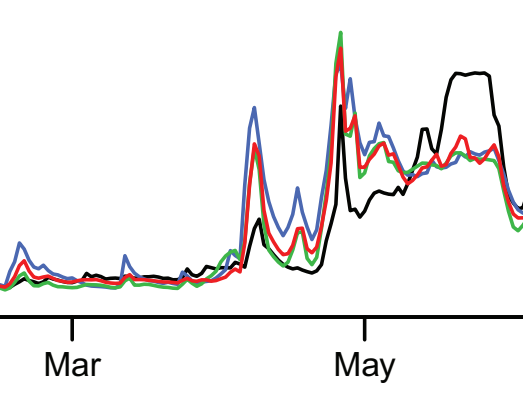

Year 1987
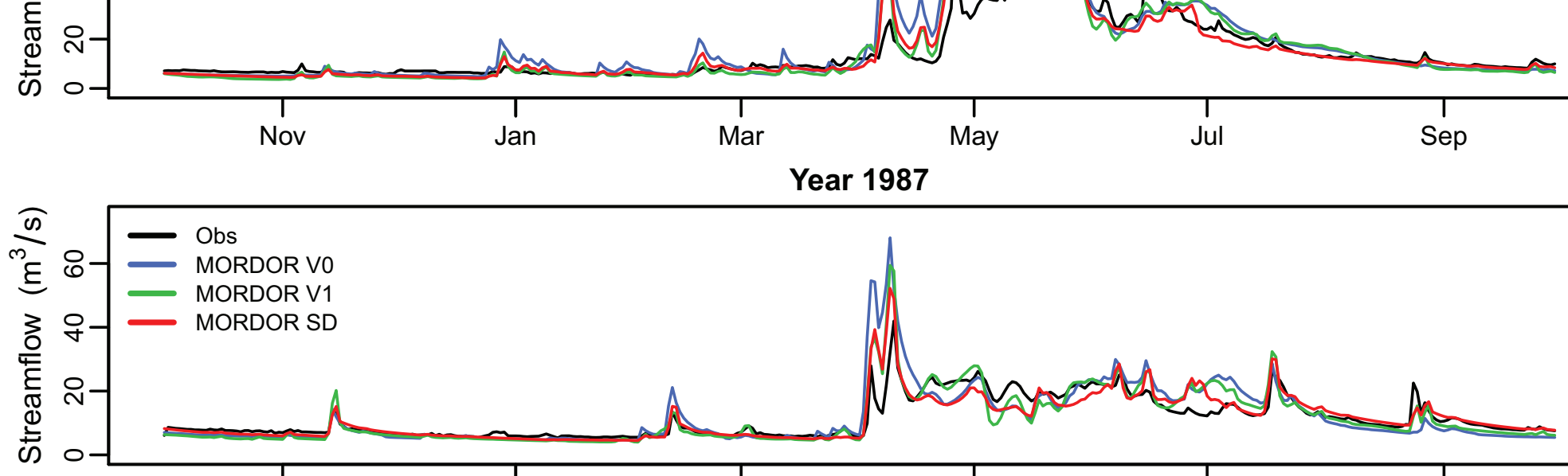


\section{Tinee@PontLune}

Year 1988

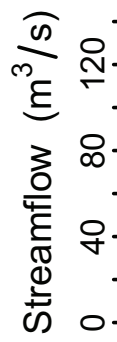

E

RDOR V0

ORDOR V1

N4A a nandand

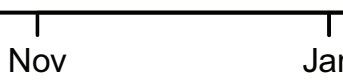

Jan Mar

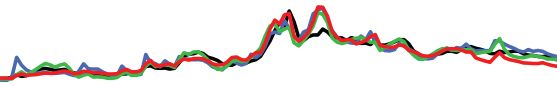

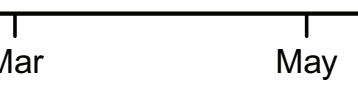

Year 1989
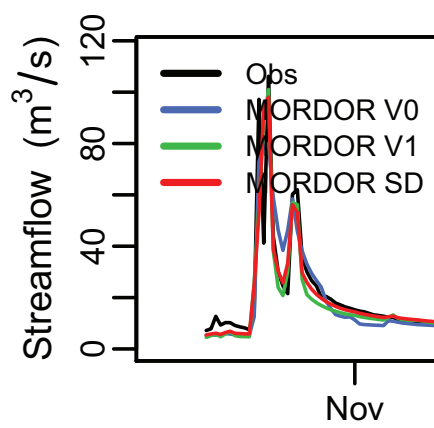

Jan

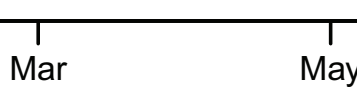

Year 1990
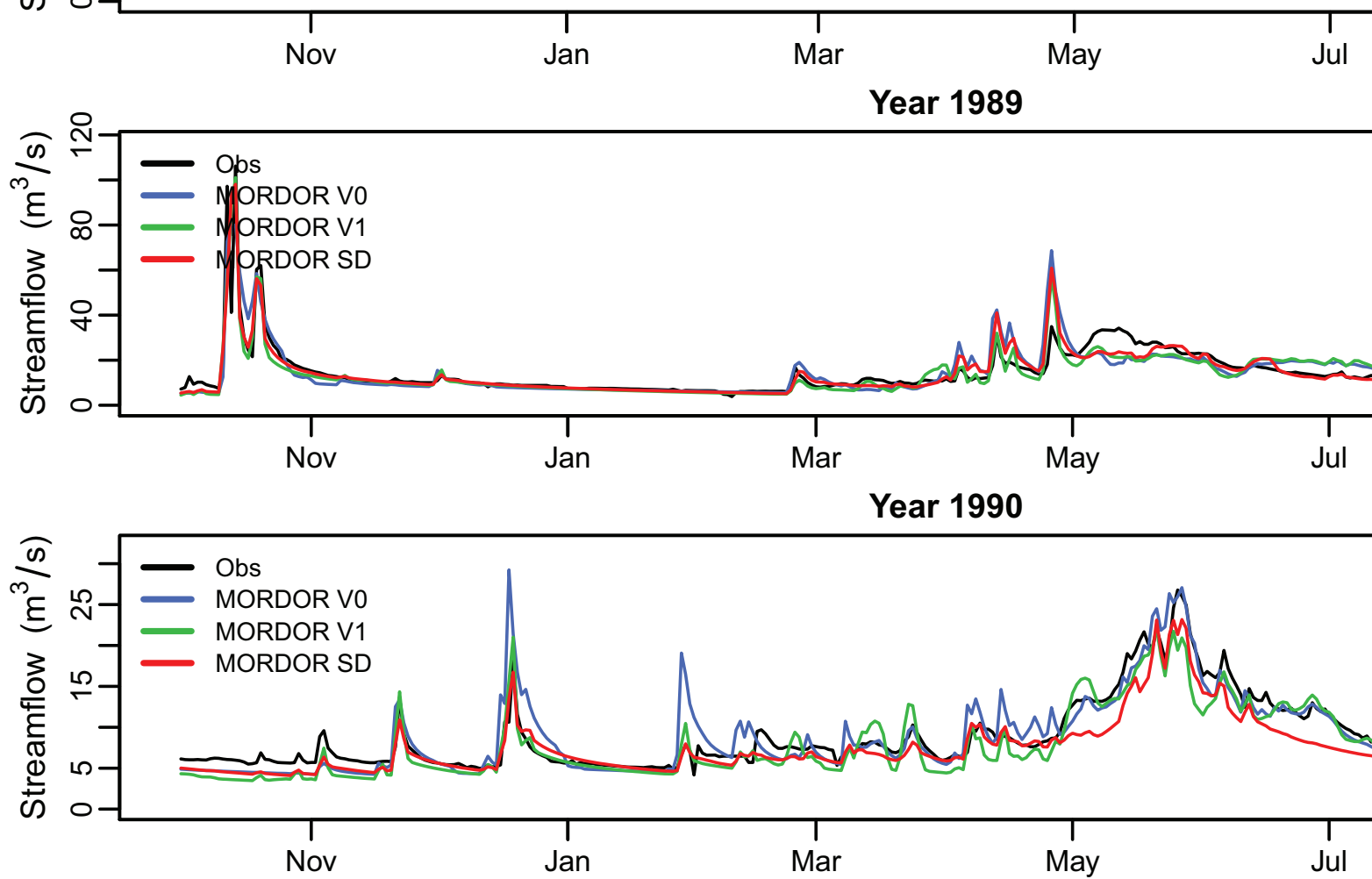

Sep

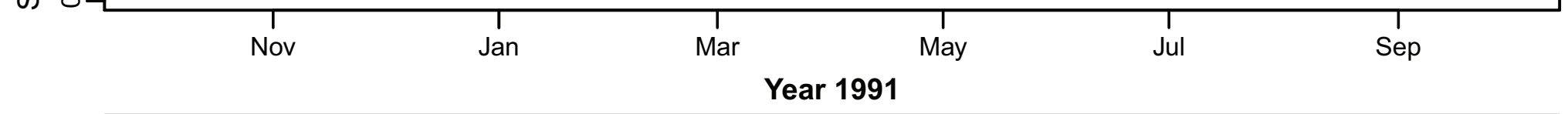

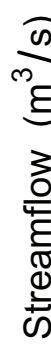
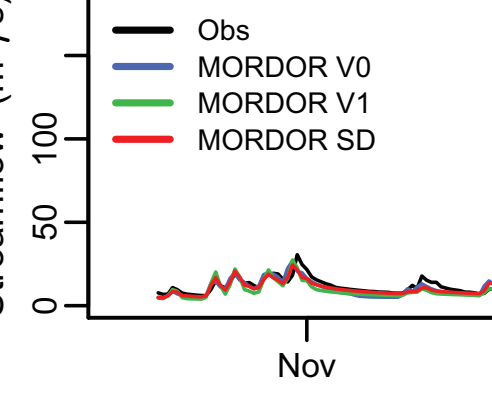

Nov

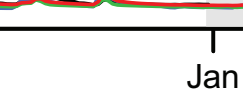

Jan
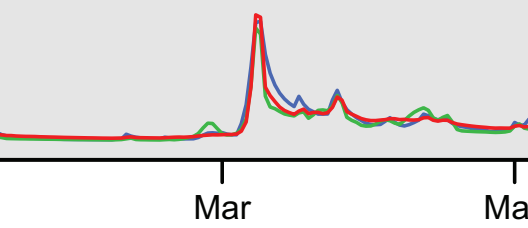

Year 1992
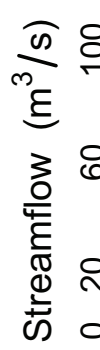

Obs
MORDOR Vo
MORDOR V1

TMORDOR SD

, w

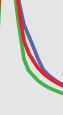

A

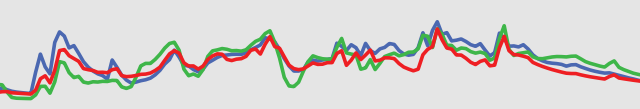

N $N$

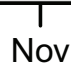

Jan

Mar

May

Jul

Sep

Year 1993
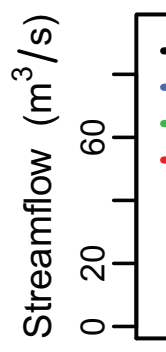

- Obs

- MORDOR V0

- MORDOR V1

- MORDOR SD

ander 


\section{Truyere@Grandval}

Year 1974

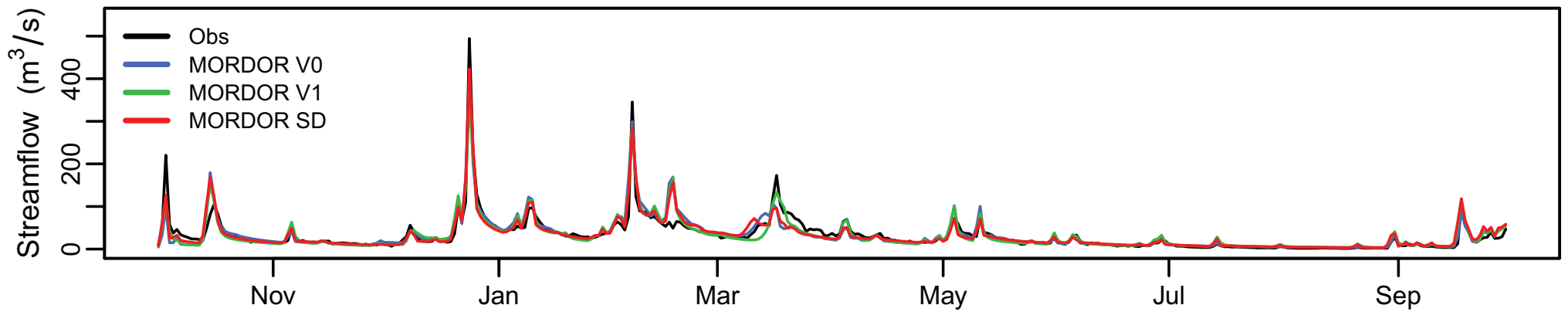

Year 1975

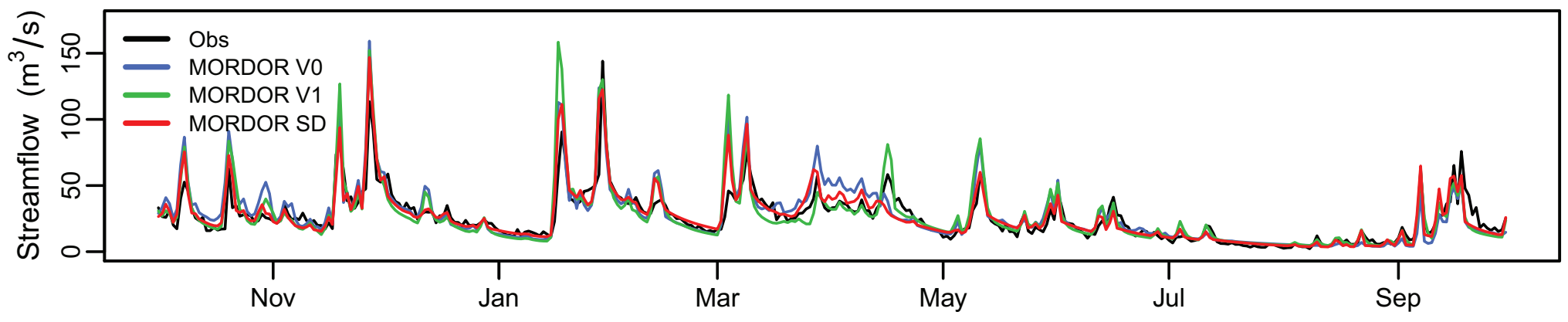

Year 1976
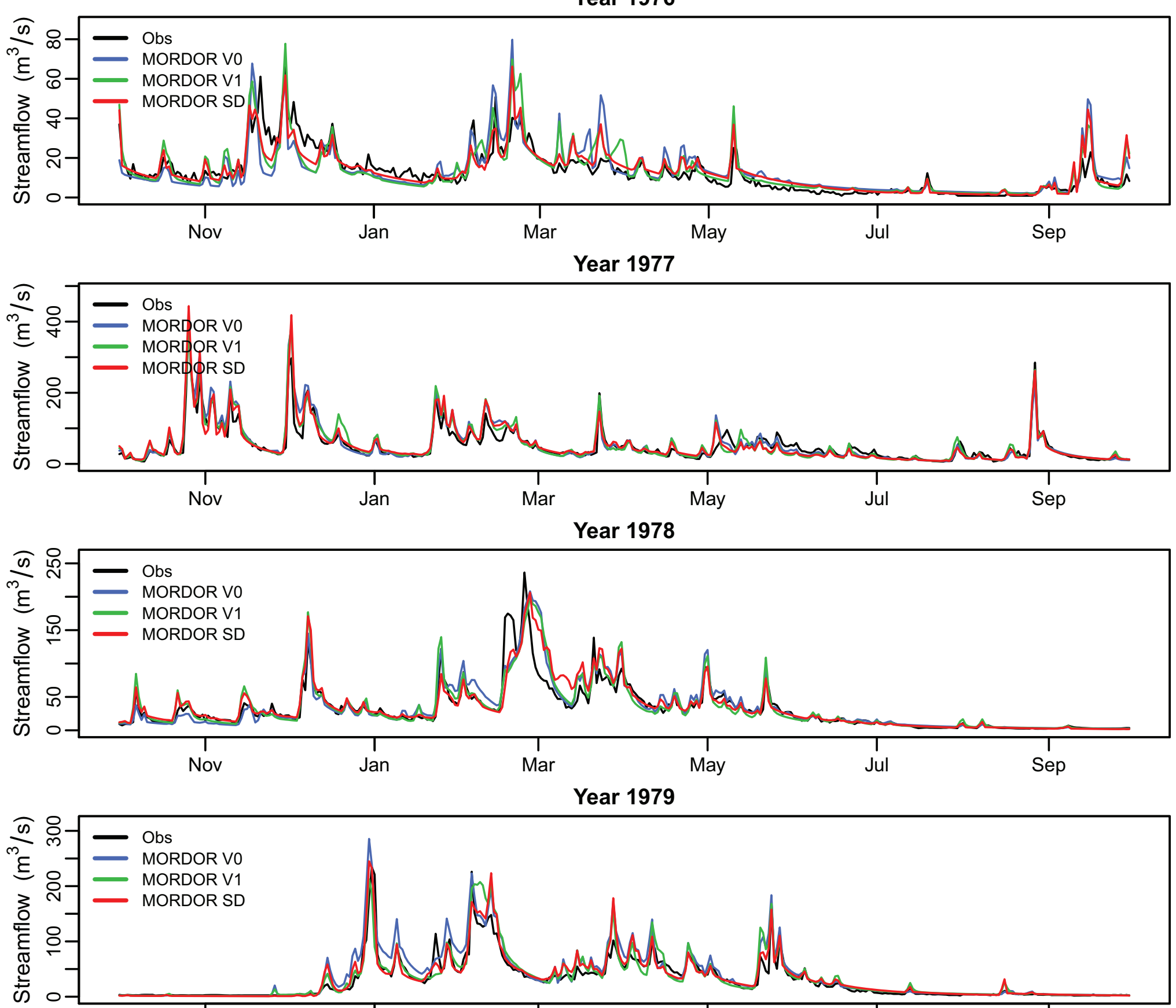


\section{Truyere@Grandval}

Year 1980

on

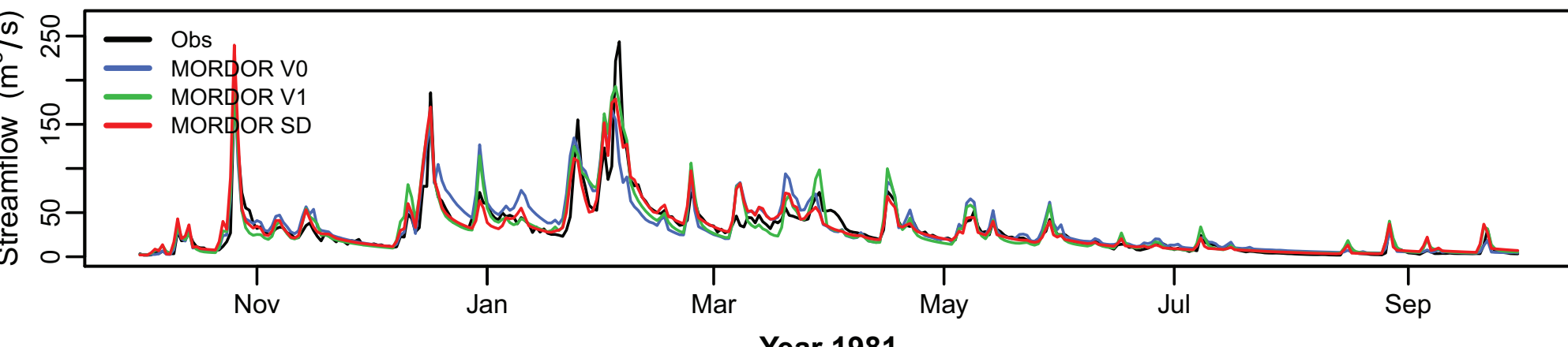

Year 1981
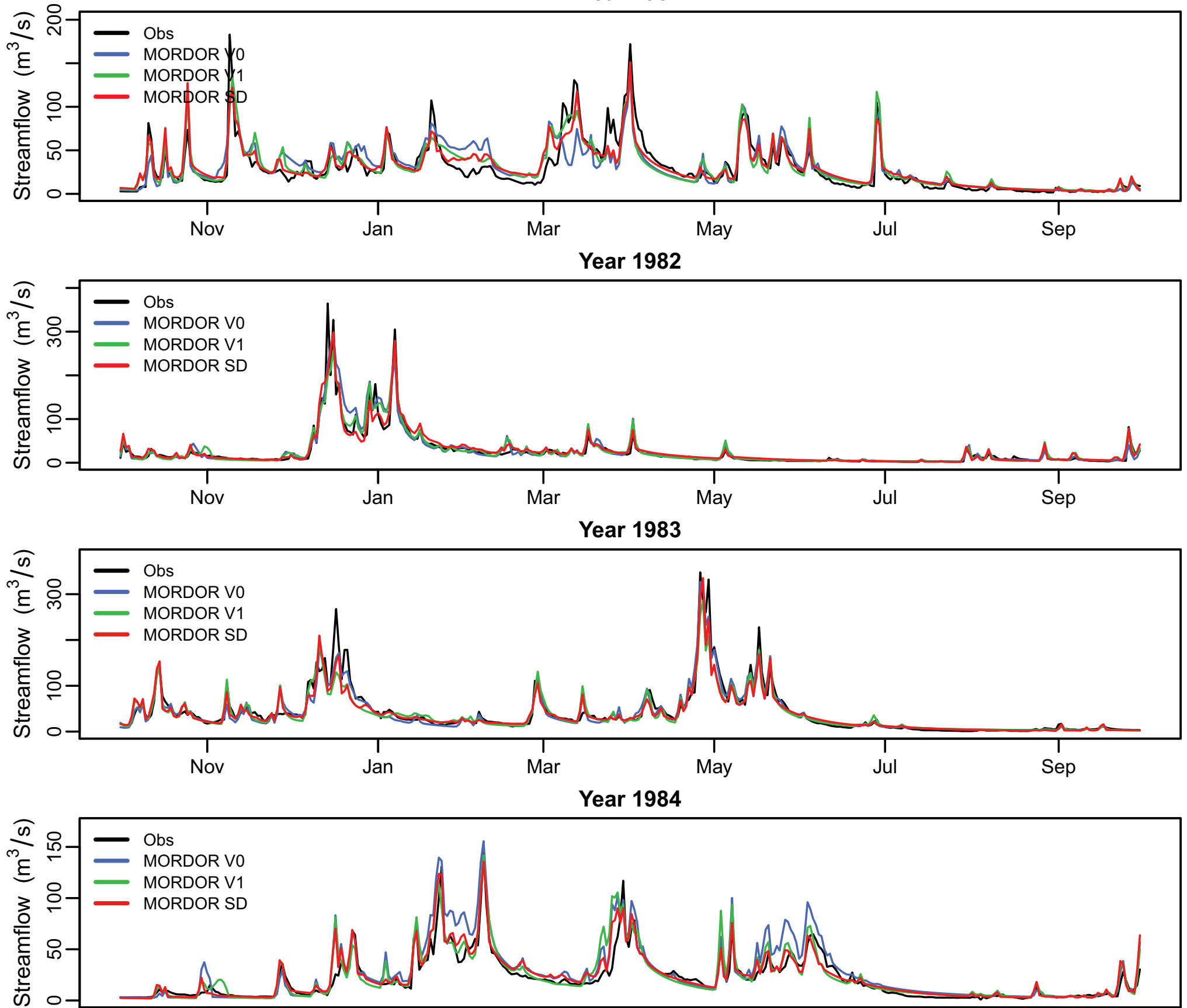


\section{Truyere@Grandval}

Year 1998
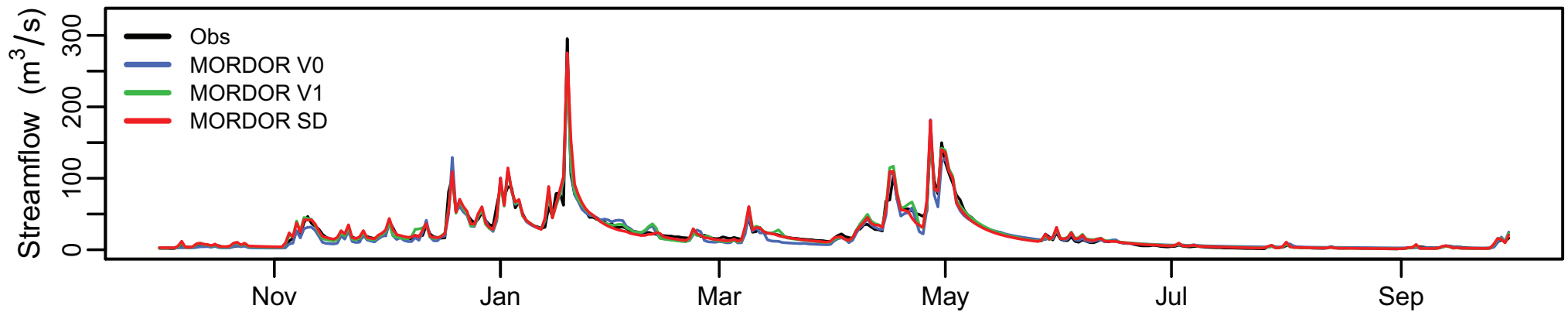

Year 1999

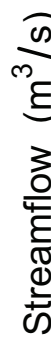

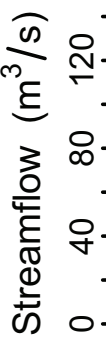

- Obs

- MORDOR V0

MORDOR V1
MORDOR SD

MORDOR V1
MORDOR SD
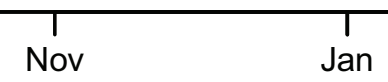

Mar

Year 2000

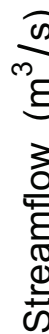

- Obs

- MORDOR V1

MORDOR SD

ㅇ--

- wathonda Ma Nov

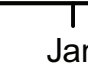

Jan Mar

Year 2001

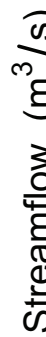

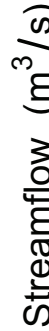

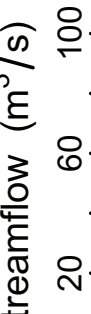

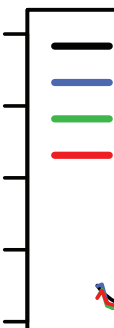

Obs

MORDOR/VO

- MORDOR 1

MORDOR $\$$
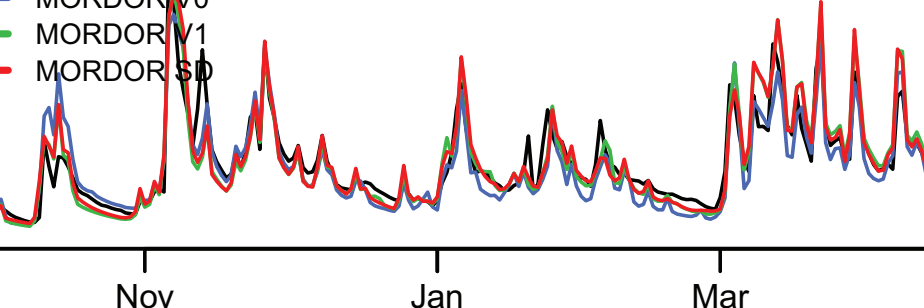

Jan

Mar May

Year 2002
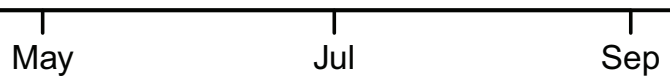
Vienne@Bussy

Year 1996

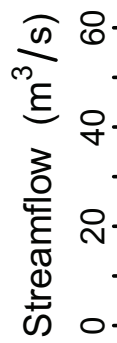

三人

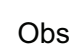

MORDOR VO

- MORDOR V1

MORDOR SD

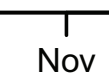

Jan

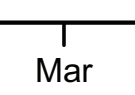

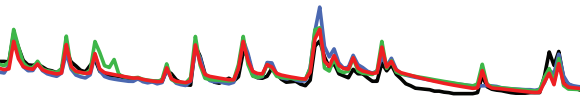

Year 1997

क

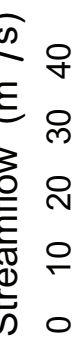

- Obs

- MORDOR V1

MORDOR SD

hum

Nov

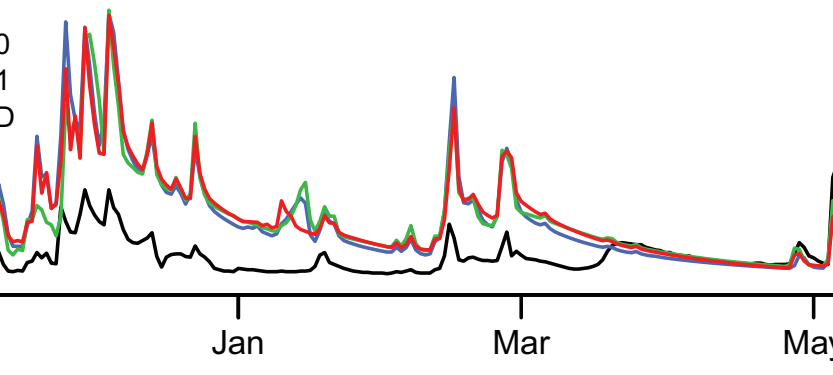

Year 1998

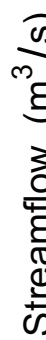

$\begin{array}{ll}- & \text { Obs } \\ - & \text { MORDOR V0 } \\ \text { MORDOR V1 }\end{array}$

ㅇ- MORDOR SD

尺-

$-1$ cha MaraNAN Nov
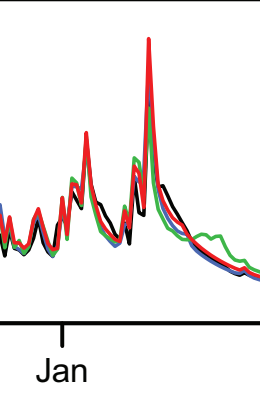

Mar

Year 1999

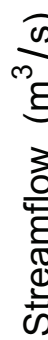

$-\begin{array}{ll}- \text { Obs } \\ - \text { MORDOR V0 } \\ \text { MORDOR V1 }\end{array}$

ㄱ- - MORDOR SD

으

An morte

Num and ind of

May

Jul

m 
